\title{
Perioperative physiology and optimalisation in bariatric and metabolic surgery
}

Citation for published version (APA):

Pouwels, J. H. (2017). Perioperative physiology and optimalisation in bariatric and metabolic surgery. [Doctoral Thesis, Maastricht University]. Maastricht University. https://doi.org/10.26481/dis.20170531sp

Document status and date:

Published: 01/01/2017

DOI:

10.26481/dis.20170531sp

Document Version:

Publisher's PDF, also known as Version of record

\section{Please check the document version of this publication:}

- A submitted manuscript is the version of the article upon submission and before peer-review. There can be important differences between the submitted version and the official published version of record.

People interested in the research are advised to contact the author for the final version of the publication, or visit the DOI to the publisher's website.

- The final author version and the galley proof are versions of the publication after peer review.

- The final published version features the final layout of the paper including the volume, issue and page numbers.

Link to publication

\footnotetext{
General rights rights.

- You may freely distribute the URL identifying the publication in the public portal. please follow below link for the End User Agreement:

www.umlib.nl/taverne-license

Take down policy

If you believe that this document breaches copyright please contact us at:

repository@maastrichtuniversity.nl

providing details and we will investigate your claim.
}

Copyright and moral rights for the publications made accessible in the public portal are retained by the authors and/or other copyright owners and it is a condition of accessing publications that users recognise and abide by the legal requirements associated with these

- Users may download and print one copy of any publication from the public portal for the purpose of private study or research.

- You may not further distribute the material or use it for any profit-making activity or commercial gain

If the publication is distributed under the terms of Article $25 \mathrm{fa}$ of the Dutch Copyright Act, indicated by the "Taverne" license above, 


\section{PERIOPERATIVE PHYSIOLOGY and OPTIMALISATION in BARIATRIC and METABOLIC SURGERY}

J.H. Pouwels 
The printing of this thesis was supported by:
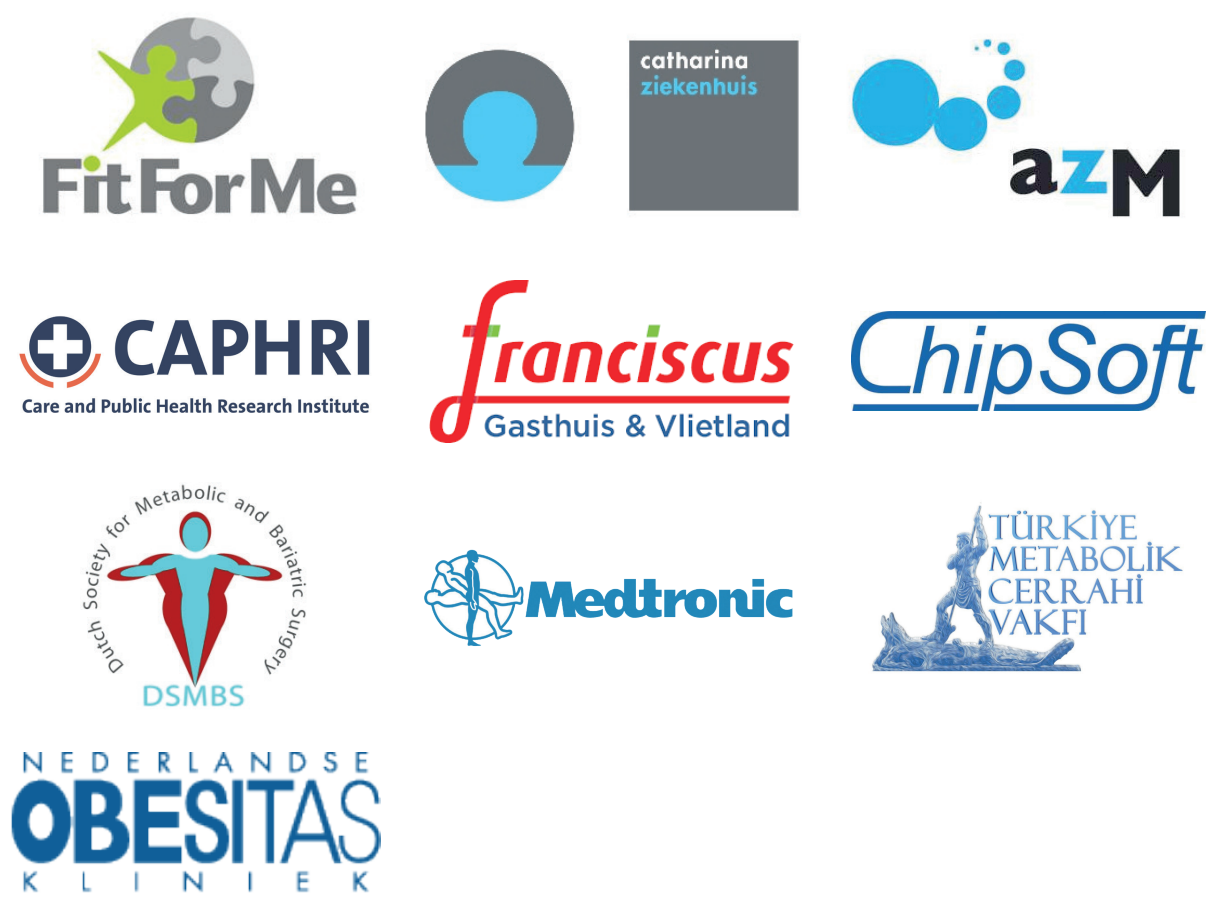

Dit proefschrift is tevens op persoonlijke titel gesponsord door B. Topal

\section{COLOFON}

(c) J.H. Pouwels, Rotterdam, the Netherlands, 2017. All rights reserved. No part of this book may be reproduced or transmitted in any form by any means, without prior permission of the author

Cover \& layout: Design Your Thesis, www.designyourthesis.com

Printing: $\quad$ Ridderprint, www.ridderprint.nl 


\title{
PERIOPERATIVE PHYSIOLOGY AND OPTIMALISATION IN BARIATRIC AND METABOLIC SURGERY
}

\author{
PROEFSCHRIFT \\ ter verkrijging van de graad van doctor \\ aan de Universiteit Maastricht, \\ op gezag van Rector Magnificus, prof. dr. Rianne M. Letschert \\ volgens het besluit van het College van Decanen, \\ in het openbaar te verdedigen \\ op woensdag 31 mei 2017 om 14.00 uur
}

door

Jacobus Hendrikus Pouwels

geboren te Amsterdam op 11 december 1989 


\section{PROMOTOR}

Prof. dr. J.A.W. Teijink

\section{COPROMOTOREN}

Dr. S.W. Nienhuijs (Catharina Ziekenhuis, Eindhoven)

Dr. M.P. Buise (Catharina Ziekenhuis, Eindhoven)

\section{BEOORDELINGSCOMMISSIE}

Prof. dr. N.D. Bouvy (voorzitter)

Prof. dr. W.F.F.A. Buhre

Prof. dr. C. Rosman (Radboudumc, Nijmegen)

Prof. dr. V. Scharnhorst (Catharina Ziekenhuis, Eindhoven)

\section{PARANIMFEN}

Ben Verkerk

Sanne Tolk 
'So that one may walk in peace' Imi Lichtenfeld (Founder of Krav Maga) 


\section{TABLE OF CONTENTS}

1. General introduction $\quad 11$

1.1 Thesis outline 14

$\begin{array}{ll}1.2 \text { References } & 16\end{array}$

\section{PART I: CARDIAC AND PULMONARY PHYSIOLOGY}

2. Cardiac structure and function before and after bariatric surgery; a new perspective

Submitted to European Journal Of Preventive Cardiology

2.1 Introduction

2.2 Prior to bariatric surgery: 'The obese'

2.3 After bariatric surgery: The "not so" obese $\quad 27$

2.4 Implications for clinical practice $\quad 31$

2.5 Recommendations $\quad 33$

2.6 Conclusion $\quad 33$

2.7 Cardiac remodelling definitions $\quad 34$

$\begin{array}{ll}2.8 \text { References } & 37\end{array}$

3. Validation of the Nexfin ${ }^{\circledR}$ non-invasive continuous blood pressure monitoring validated against Riva-Rocci/Korotkoff in a bariatric patient population $\quad 43$

Accepted for publication in Journal Of Clinical Anesthesia

3.1 Introduction $\quad 45$

3.2 Methods $\quad 46$

3.3 Results 49

3.4 Discussion $\quad 54$

3.5 Conclusion $\quad 56$

$\begin{array}{ll}3.6 \text { References } & 57\end{array}$

4. Short-term changes in cardiovascular hemodynamics in response to bariatric surgery and weight loss using the Nexfin ${ }^{\circledR}$ non-invasive continuous monitoring device: A pilot study

Accepted for publication in Obesity Surgery

4.1 Introduction

4.2 Methods

4.3 Results

4.4 Discussion 
$\begin{array}{ll}4.5 \text { Conclusion } & 70\end{array}$

4.6 References $\quad 71$

5. Perioperative respiratory care in obese patients undergoing bariatric surgery: implications for clinical practice

Respir Med. 2016 Aug.117:73-80

5.1 Introduction $\quad 79$

$\begin{array}{ll}5.2 \text { Respiratory physiology in the obese } & 79\end{array}$

5.3 Perioperative respiratory care in bariatric surgery $\quad 84$

5.4 Is preoperative pulmonary function testing necessary in bariatric surgery? $\quad 87$

5.5 Conclusion $\quad 90$

5.6 References $\quad 91$

6. Effects of bariatric surgery on inspiratory muscle strength 99

Springerplus. 2015 Jul7.4:322-329.

6.1 Introduction $\quad 101$

$\begin{array}{ll}\text { 6.2 Methods } & 101\end{array}$

$\begin{array}{ll}6.3 \text { Results } & 103\end{array}$

$\begin{array}{ll}\text { 6.4 Discussion } & 105\end{array}$

$\begin{array}{ll}6.5 \text { Conclusion } & 109\end{array}$

$\begin{array}{ll}6.6 \text { References } & 110\end{array}$

7. Comparative analysis of respiratory muscle strength before and after bariatric $\begin{array}{ll}\text { surgery using five different predictive equations } & 115\end{array}$

JClin Anesth. 2016 Aug.32:172-80

$\begin{array}{ll}7.1 \text { Introduction } & 117\end{array}$

$\begin{array}{ll}7.2 \text { Methods } & 119\end{array}$

$\begin{array}{ll}7.3 \text { Results } & 121\end{array}$

$\begin{array}{ll}7.4 \text { Discussion } & 126\end{array}$

$\begin{array}{ll}7.5 \text { Conclusion } & 129\end{array}$

$\begin{array}{ll}7.6 \text { References } & 130\end{array}$

8. Aspects of exercise before and after bariatric surgery: a systematic review 135 Obes Facts 2015, 8(2): 132-146

$\begin{array}{ll}8.1 \text { Introduction } & 137\end{array}$

$\begin{array}{ll}\text { 8.2 Methods } & 137\end{array}$

$\begin{array}{ll}8.3 \text { Results } & 139\end{array}$

8.4 Discussion 150

8.5 Conclusion 153

8.6 References 154 


\section{PART II: METABOLIC PHYSIOLOGY}

9. Different supplementation regimes to treat perioperative vitamin B12 deficiencies in bariatric surgery: a systematic review

Obes Surg. 2017 Jan.27(1):254-262

9.1 Introduction

9.2 Methods

9.3 Results

9.4 Discussion

9.5 Conclusion

9.6 References

10. Comparison between different intramuscular vitamin B12 supplementation regimes: A retrospective matched cohort study

Obes Surg. 2016 Dec. 26(12): 2873-2879

10.1 Introduction

10.2 Methods

10.3 Results

10.4 Discussion

10.5 Conclusion

10.6 References

11. Improved and more effective algorithms to screen for nutrient deficiencies after bariatric surgery

Eur J Clin Nutr. 2017 Feb.71(2):198-202

11.1 Introduction

11.2 Methods

11.3 Results

11.4 Discussion

11.5 Conclusion

11.6 References

12. Time to glycemic control: an observational study of $\mathbf{3}$ different operations ObesSurg. 2017 Mar.27(3):694-702

12.1 Introduction

12.2 Methods

12.3 Results

12.4 Discussion

12.5 Conclusion 
13. Summary, discussion and future perspectives

14. Valorisation

15. Nederlandse samenvatting

16. Dankwoord

17. Curriculum vitae

275

18. List of publications

279 


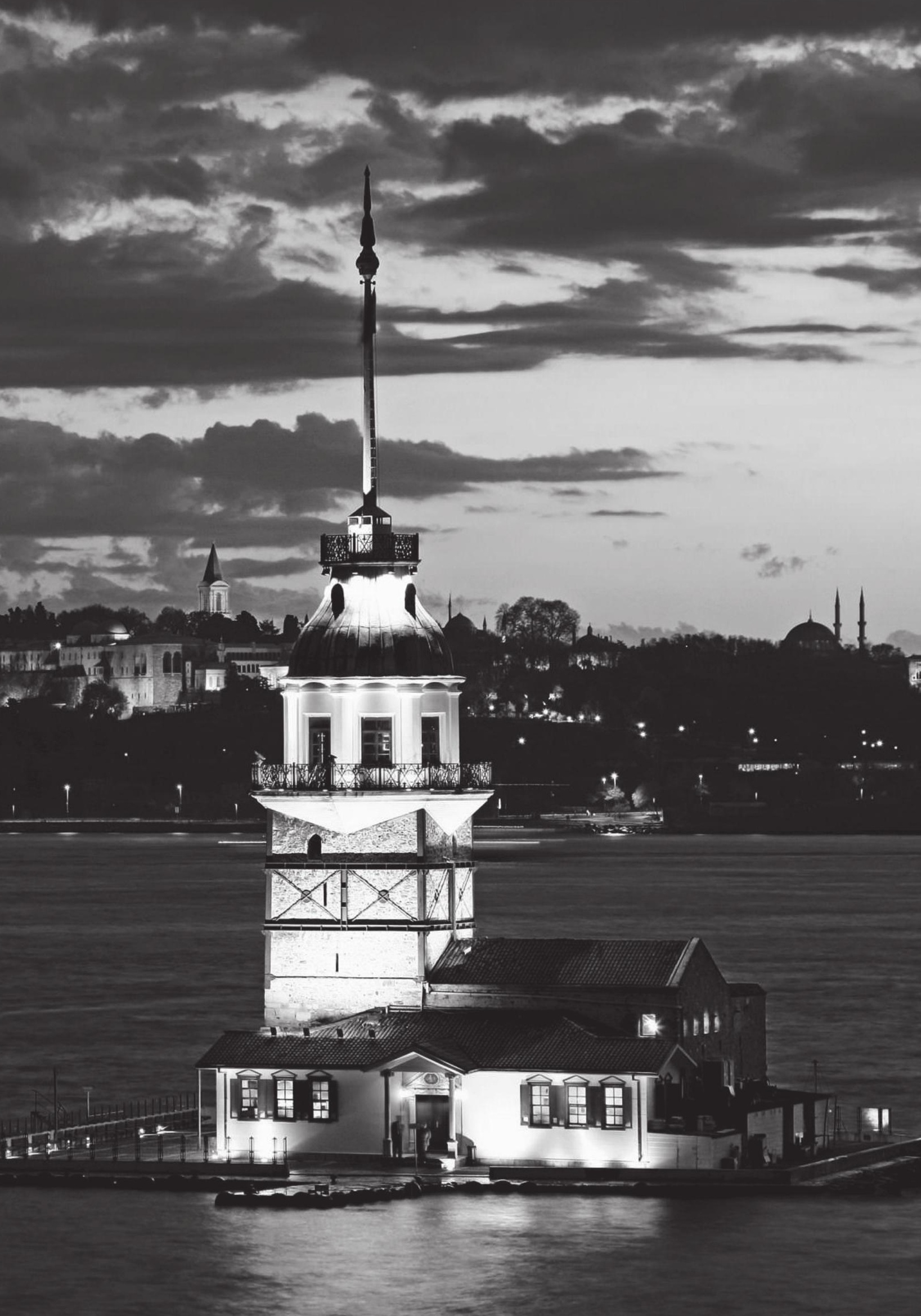


1. GENERAL INTRODUCTION 

The obesity pandemic is reaching epic proportions (1) and bariatric surgery is the most effective long-term intervention for obesity. The National Institutes Of Health (NIH) consensus statement published 22 years ago largely governed the use of bariatric surgery worldwide. (2) Patients with a Body Mass Index (BMI) greater than $40 \mathrm{~kg} / \mathrm{m}^{2}$, or greater than $35 \mathrm{~kg} / \mathrm{m}^{2}$ with obesity related comorbidities such as type 2 diabetes, are eligible for bariatric surgery. Although these recommendations were carefully written and based on evidence (available at that time), they are outdated and have important limitations. $(3,4)$

Anno 2016 this NIH statement is still used as guideline, but bariatric surgery is shifting to a more metabolic approach. Even the name 'bariatric surgery' might have to be changed to metabolic surgery. Nowadays there is more and more evidence present that the metabolic profile of patients need a selection criterion for surgery. It has become increasingly apparent that bariatric surgery exerts powerful, beneficial effects on metabolic diseases, such as type 2 diabetes mellitus (T2DM) (4-8), but also pulmonary and cardiac diseases, like asthma $(9,10)$ and heart failure. $(11,12)$ The only landmark study in bariatric and metabolic surgery, the Swedish Obese Subjects study has shown that bariatric surgery is capable of major reductions in cardiovascular risk factors, events and even mortality. (13)

The curiosity about the physiological mechanisms of bariatric and metabolic surgery is not a new phenomenon. The notion that rerouting of the gastro-intestinal tract alters glycaemia was first described in 1930 after surgery for peptic ulcer disease. (14) Bariatric surgical procedures were developed in the 1950's for weight loss primarily, but several reports showed that there was a rapid reduction of T2DM. (14) Pories et al. reported a T2DM reversal in $78 \%$ patients who underwent gastric bypass surgery, and proposed that T2DM might even be a surgical disease. $(5,15)$ Historically, bariatric surgery was meant to promote weight loss by causing gastric restriction and malabsorption. $(5,15)$ However, newer mechanistic/ physiological studies, in parallel with the establishment that the gastro-intestinal tract is a primary regulator of the glucose homeostasis, showed that there is 'more than meets the eye'. Discrete parts of the gastro-intestinal tract have unique physiological functions; hence, the underlying mechanisms contributing to improved glucose homeostasis, clinical outcomes and physiological changes differ among anatomical procedures. $(5,7,8,15)$ Indeed, T2DM remission rates differ according to the type of surgery, with the lowest remission rates in purely restrictive procedures (such as laparoscopic gastric banding (LAGB)) and the highest rates in malabsorptive procedures, as reported by Buchwald et al. (8)

With increasing understanding of the physiology of the T2DM remission, more insights were also created regarding other physiological changes. One of those is the prevalence of nutrient deficiencies and how to optimise a patient prior to specific bariatric surgical procedures (1618), but also the body of literature regarding cardiac (19) and pulmonary physiology $(9,10,20)$ after bariatric surgery is growing. Moreover, the physiological mechanisms of the bariatric 
surgery on the amelioration of type 2 diabetes, cardiovascular and pulmonary diseases are thought to be weight-dependent but also weight independent (13) The thesis presented here will give insights about some aspects of cardiopulmonary and metabolic physiology following bariatric and metabolic surgery.

\subsection{THESIS OUTLINE}

In this thesis, several aspects of physiology following bariatric and metabolic surgery are described. It contains both clinical and literature-based research. The content of this thesis is divided in two parts. The first part describes the cardiopulmonary physiology after bariatric and metabolic surgery. The second part elaborates on two aspects of metabolic physiology: nutrient deficiencies and their treatment, and the remission of type 2 diabetes.

\section{PART I: CARDIAC AND PULMONARY PHYSIOLOGY}

Chapter 2 gives an overview of the cardiac structure and function changes before and after bariatric surgery. In chapter 3 , the Nexfin ${ }^{\circledR}$ noninvasive continuous hemodynamic monitoring device was validated against blood pressure measurement according to RivaRocci/Korotkoff in obese patients scheduled for bariatric surgery. The results of a prospective cohort study investigating hemodynamic changes before and after surgery using the Nexfin ${ }^{\circledast}$ device are described in chapter 4 .

The pulmonary physiology in patients with obesity and its considerations for perioperative care in bariatric surgery are described in chapter 5 . Changes in maximal inspiratory muscle strength are one of the important pulmonary function alterations after bariatric surgery (chapter 6), but are very difficult to predict with the current available predictive equations. Chapter 7 gives a critical appraisal of the available predictive equations. In the final chapter of part I (chapter 8), the current evidence regarding perioperative exercise in bariatric surgery was systematically reviewed and this chapter an exercise advice was given.

\section{PART II: METABOLIC PHYSIOLOGY}

In chapter 9, the variations in supplementation regimes to treat perioperative vitamin B12 deficiencies were systematically reviewed and analysed. Chapter 10 is a retrospective matched cohort study to assess the effectiveness of three different intramuscular vitamin B12 supplementation regimes. Nowadays, nutrient deficiencies are quite common after bariatric surgery, but there seems to be differences in the deficiencies after several surgical procedures. In Chapter 11, we aimed to develop an algorithm to improve and optimize the screening for 
nutrient deficiencies after bariatric surgery. The final chapter of part II (chapter 12) assessed the time to glycemic control after several bariatric surgical procedures. 


\subsection{REFERENCES}

1. Ng M, Fleming T, Robinson M, Thomson B, Graetz N, Margono C, et al. Global, regional, and national prevalence of overweight and obesity in children and adults during 1980-2013: a systematic analysis for the Global Burden of Disease Study 2013. Lancet (London, England). 2014;384(9945):766-81.

2. NIH conference. Gastrointestinal surgery for severe obesity. Consensus Development Conference Panel. Annals of internal medicine. 1991;115(12):956-61.

3. Flum DR, Belle SH, King WC, Wahed AS, Berk P, Chapman W, et al. Perioperative safety in the longitudinal assessment of bariatric surgery. The New England journal of medicine. 2009;361(5):445-54.

4. Nguyen NT, Hinojosa M, Fayad C, Varela E, Wilson SE. Use and outcomes of laparoscopic versus open gastric bypass at academic medical centers. Journal of the American College of Surgeons. 2007;205(2):248-55.

5. Pories WJ, Swanson MS, MacDonald KG, Long SB, Morris PG, Brown BM, et al. Who would have thought it? An operation proves to be the most effective therapy for adult-onset diabetes mellitus. Annals of surgery. 1995;222(3):339-50; discussion 50-2.

6. Schauer PR, Burguera B, Ikramuddin S, Cottam D, Gourash W, Hamad G, et al. Effect of laparoscopic Roux-en Y gastric bypass on type 2 diabetes mellitus. Annals of surgery. 2003;238(4):467-84; discussion 84-5.

7. Buchwald H, Avidor Y, Braunwald E, Jensen MD, Pories W, Fahrbach K, et al. Bariatric surgery: a systematic review and meta-analysis. Jama. 2004;292(14):1724-37.

8. Buchwald H, Estok R, Fahrbach K, Banel D, Jensen MD, Pories WJ, et al. Weight and type 2 diabetes after bariatric surgery: systematic review and meta-analysis. The American journal of medicine. 2009;122(3):248-56 e5.

9. van Huisstede A, Rudolphus A, Castro Cabezas M, Biter LU, van de Geijn GJ, Taube C, et al. Effect of bariatric surgery on asthma control, lung function and bronchial and systemic inflammation in morbidly obese subjects with asthma. Thorax. 2015;70(7):659-67.

10. van Huisstede A, Rudolphus A, van Schadewijk A, Cabezas MC, Mannaerts GH, Taube C, et al. Bronchial and systemic inflammation in morbidly obese subjects with asthma: a biopsy study. American journal of respiratory and critical care medicine. 2014;190(8):951-4.

11. Jeng EI, Aranda JM, Jr., Ahmed M, Klodell CT. Left Ventricular Assist Device and Bariatric Surgery: A Bridge to Heart Transplant by Weight and Waiting Time Reduction. Journal of cardiac surgery. 2016;31(2):120-2.

12. Lim CP, Fisher OM, Falkenback D, Boyd D, Hayward CS, Keogh A, et al. Bariatric Surgery Provides a "Bridge to Transplant" for Morbidly Obese Patients with Advanced Heart Failure and May Obviate the Need for Transplantation. Obesity surgery. 2016;26(3):486-93.

13. Sjostrom L, Peltonen M, Jacobson P, Sjostrom CD, Karason K, Wedel H, et al. Bariatric surgery and long-term cardiovascular events. Jama. 2012;307(1):56-65.

14. Batterham RL, Cummings DE. Mechanisms of Diabetes Improvement Following Bariatric/ Metabolic Surgery. Diabetes care. 2016;39(6):893-901. 
15. Pories WJ, MacDonald KG, Jr., Flickinger EG, Dohm GL, Sinha MK, Barakat HA, et al. Is type II diabetes mellitus (NIDDM) a surgical disease? Annals of surgery. 1992;215(6):633-42; discussion 43.

16. Weng TC, Chang CH, Dong YH, Chang YC, Chuang LM. Anaemia and related nutrient deficiencies after Roux-en-Y gastric bypass surgery: a systematic review and meta-analysis. BMJ open. 2015;5(7):e006964.

17. Aron-Wisnewsky J, Verger EO, Bounaix C, Dao MC, Oppert JM, Bouillot JL, et al. Nutritional and Protein Deficiencies in the Short Term following Both Gastric Bypass and Gastric Banding. PloS one. 2016;11(2):e0149588.

18. Verger EO, Aron-Wisnewsky J, Dao MC, Kayser BD, Oppert JM, Bouillot JL, et al. Micronutrient and Protein Deficiencies After Gastric Bypass and Sleeve Gastrectomy: a 1-year Follow-up. Obesity surgery. 2016;26(4):785-96.

19. Alpert MA. Cardiac morphology and performance in severe obesity: size really does matter. Cardiology. 2014;127(3):141-3.

20. van Huisstede A, Biter LU, Luitwieler R, Castro Cabezas M, Mannaerts G, Birnie E, et al. Pulmonary function testing and complications of laparoscopic bariatric surgery. Obesity surgery. 2013;23(10):1596-603. 

PART I: CARDIAC AND PULMONARY PHYSIOLOGY 


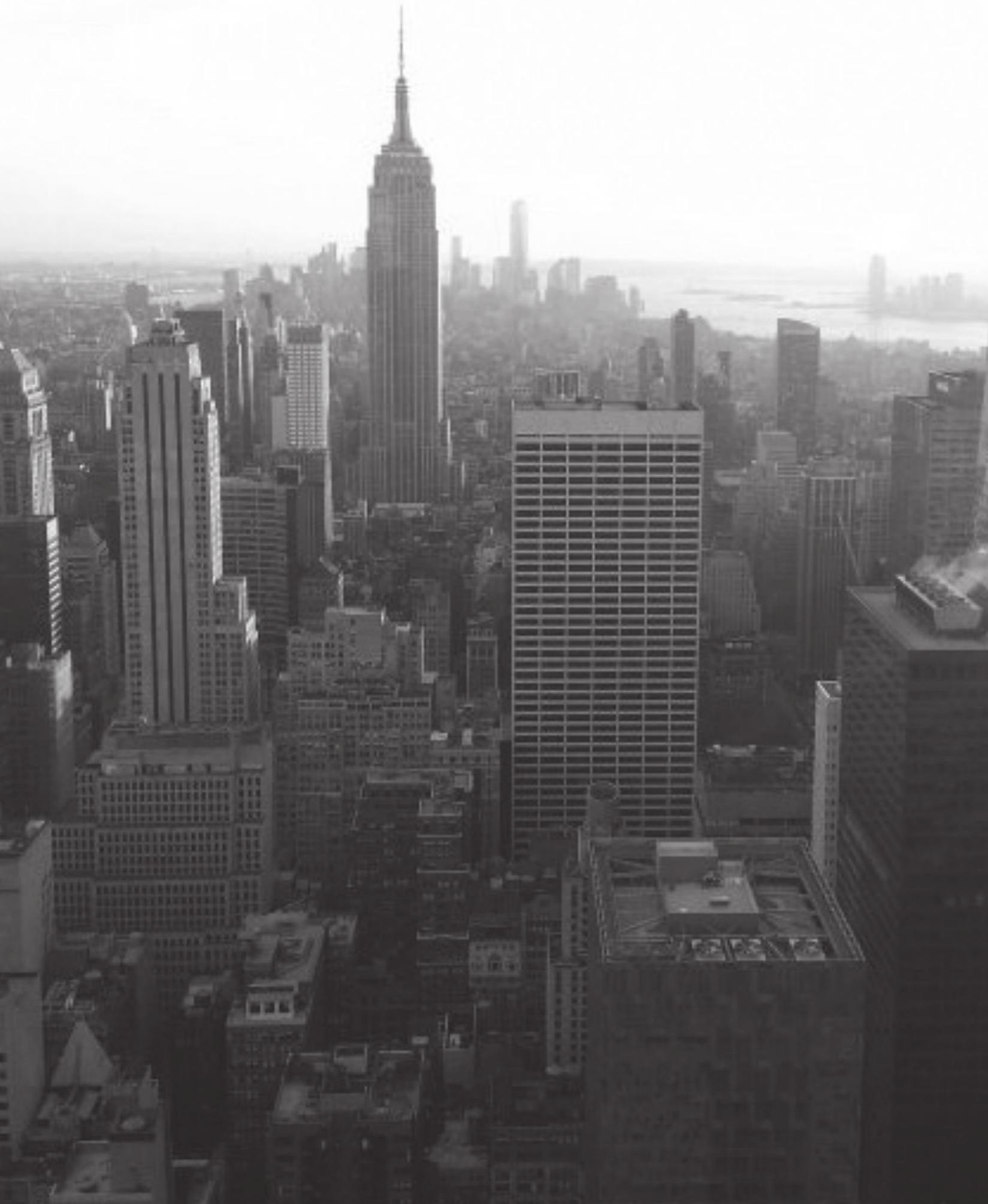




\title{
2. CARDIAC STRUCTURE AND FUNCTION BEFORE AND AFTER BARIATRIC SURGERY; A NEW PERSPECTIVE
}

\author{
Bianca Lascaris ${ }^{1}$ \\ Sjaak Pouwels ${ }^{2,3}$ \\ Patrick Houthuizen ${ }^{4}$ \\ Lukas R. Dekker ${ }^{4}$ \\ Simon W. Nienhuijs ${ }^{3}$ \\ R. Arthur Bouwman ${ }^{1}$ \\ Marc P. Buise ${ }^{1}$
}

Submitted to European Journal Of Preventive Cardiology

1. Department of Anaesthesiology, Intensive Care and Pain Medicine, Catharina Hospital, Eindhoven, The Netherlands

2. Department of Epidemiology, CAPHRI Research School, Maastricht University, The Netherlands 3. Department of Surgery, Catharina Hospital, Eindhoven, The Netherlands 4. Department of Cardiology, Catharina Hospital, Eindhoven, The Netherlands 


\section{ABSTRACT}

Obesity, defined as a body Mass Index of $\geq 30 \mathrm{~kg} / \mathrm{m} 2$, is the most common chronic metabolic disease worldwide and its prevalence has been strongly increasing. Obesity is associated with various diseases such as cardiovascular disease, type 2 diabetes, hypertension, obstructive sleep apnoea syndrome, rheumatoid arthritis and neoplasms. Obesity has deleterious effects on cardiac function. The purpose of this review is to evaluate the cardiac function and structure changes and hemodynamic responses to obesity before and after excessive weight loss. Secondly, to elucidate the possible clinical implications of cardiac remodelling after bariatric surgery for perioperative and anesthesiological care. 


\subsection{INTRODUCTION}

Obesity, defined as a Body Mass Index (BMI) of $\geq 30 \mathrm{~kg} / \mathrm{m} 2$, is the most common chronic metabolic disease worldwide and its prevalence has been strongly increasing. (1) Obesity is associated with various diseases such as cardiovascular disease, type 2 diabetes, hypertension, obstructive sleep apnoea syndrome (OSAS), together called the metabolic syndrome (2)

Obesity has physiological effects on several organ systems, like pulmonary and cardiac function. Obesity induces pulmonary and cardiac changes that can result in right and/or left heart failure. (3) Basically, the pathophysiological mechanism is multifactorial. With moderate or severe obesity, significant increments in blood volume are found (this reflects an increased size of the vascular bed) and this paralleled with an increase in cardiac output. (3) Under this state of increased cardiac output, renal and cerebral blood flow remains roughly the same (compared to 'ideal body weight'). (3) Results from inert gas washout studies showed that excess body fat incorporates an extra blood flow of $2-3 \mathrm{ml} / \mathrm{min} / 100 \mathrm{~g}$. This means that $100 \mathrm{~kg}$ of excess body fat would require as much as $3 \mathrm{~L} / \mathrm{min}$ blood flow, that implicates an increase in cardiac output. (3) Actually, the blood volume and cardiac output of an individual of $170 \mathrm{~kg}$ are roughly twice those of a subject of $70 \mathrm{~kg}$. (3) It is a clinical observation that obese patients do not suffer tachycardia; per physiology there must be an increase in stroke volume to reach the high cardiac output. $(3,4)$

This basically means that obesity results in augmented left ventricular preload (volume) and often increased afterload (hypertension), with maintenance of a high output state at the expense of elevated right and left ventricular filling pressures. $(3,4)$

Some of the cardio-pulmonary changes to obesity are associated with obstructive sleep apnoea (OSA) and obese hypoventilation syndrome (OHS). OHS is characterised by chronic hypoxia, hypercapnia and respiratory acidosis. (3) This chronic hypoxia and hypercapnia result mainly in pulmonary vasoconstriction. (3) The presence of high pre-existing pulmonary blood flow in conjunction with pulmonary vasoconstriction lead to pulmonary hypertension and a significant trans-pulmonary diastolic pressure gradient in addition to elevated left ventricular filling pressures. $(3,4)$

A large amount of information on obesity and cardiac function is available. In this review, we will evaluate the cardiac function, structural changes and hemodynamic responses to obesity by integrating the influence of volume and pressure on both the left and the right ventricles. Secondly this review aims to elucidate the possible clinical implications of cardiac remodelling after excessive weight loss by bariatric surgery for perioperative and anesthesiological care. 


\subsection{PRIOR TO BARIATRIC SURGERY: 'THE OBESE'}

\subsubsection{CARDIAC GEOMETRY}

\section{Left ventricle}

The chronic volume overload (preload) and hypertension (afterload) in obesity, lead to compensatory mechanisms as left ventricular enlargement and hypertrophy. To determine the degree of hypertrophy, the thickness of the basal interventricular septum (IVST) and posterior wall (PWT) are measured by echocardiography; a mathematical formula is used to estimate left ventricular mass (LVM) from these measurements. (Figure 2.1). (5) An increase in wall thickness (IVST, PWT) with normal LVM is defined as concentric remodelling as opposed to concentric hypertrophy (in which LVM is also increased).(5)

Both volume-overload induced by the increased blood volume and hypertension induce left ventricular remodelling in the obese. $(4,6-8)$ Indeed, it has been observed that the left ventricle dilates in the obese, resulting in increased diastolic and systolic volumes compared to lean. (9) On the other hand, it is also reported that obesity is frequently associated with either concentric or eccentric remodelling (2.6-74\%). Figure 2.2 gives an overview of the pathophysiological changes induced by obesity on the cardiac and hemodynamic function.

\section{Right ventricle}

Although right ventricular dysfunction is more common in the obese, right ventricular (RV) function has gained much less attention than left ventricular function. One study showed an increase in right myocardial performance index, as well as isovolumetric contraction and relaxation time together with reduction RV ejection time compared to lean subjects.(10) These observations indicate both systolic and diastolic dysfunction in obese patients.

Another study showed a significant greater RV mass in obese, as well as RV end-diastolic and end-systolic volumes, and RV stroke volume (as measured by MRI), however without differences in RV ejection fraction between obese and lean.(7) This suggests a mechanism where a reduction in stroke volume (and ejection fraction) is compensated by the FrankStarling mechanism. As a result, ejection fraction remains constant at the expense of ventricular dilation. 


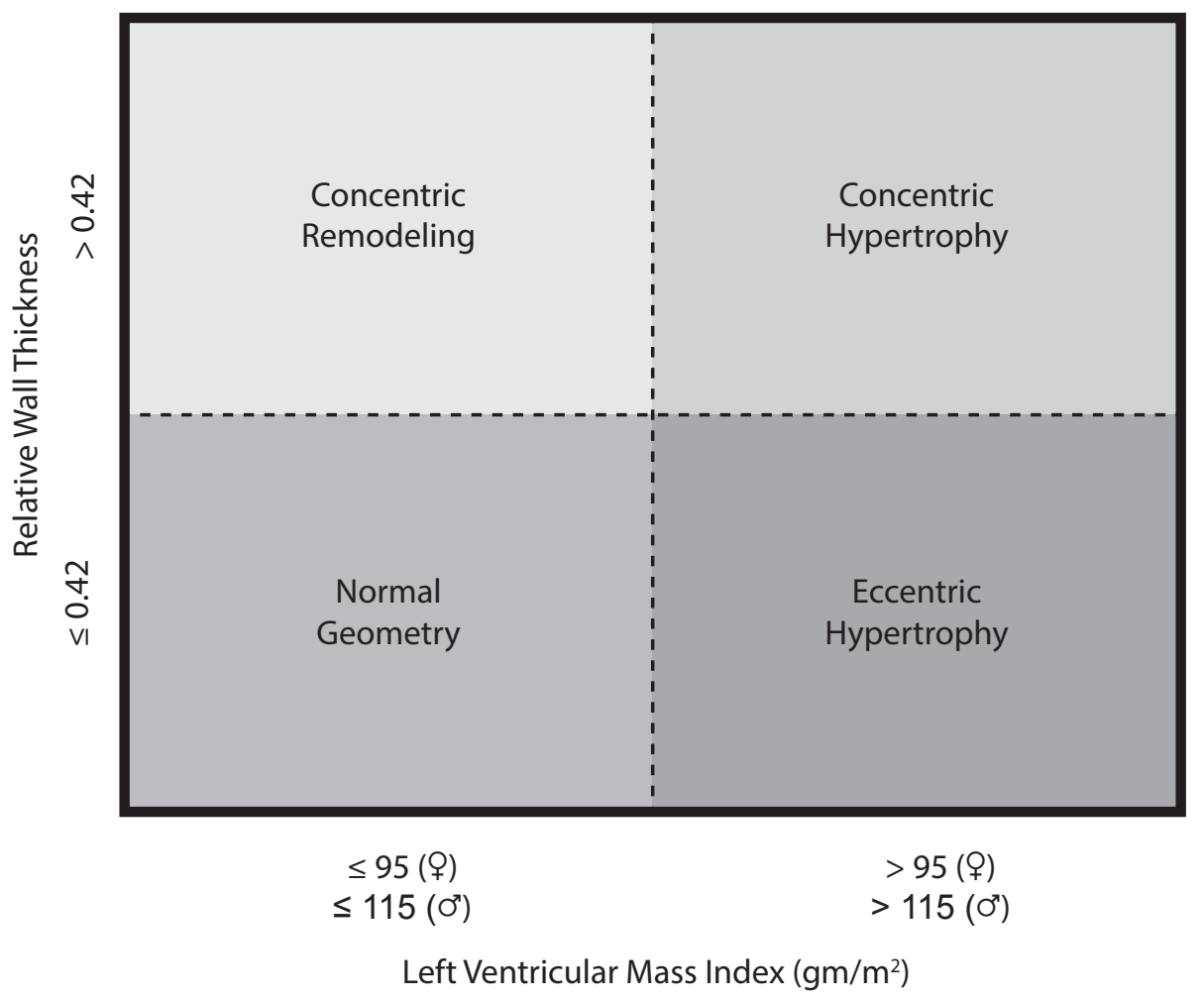

FIGURE 2.1: Comparison Relative Wall Thickness (RWT) and Left Ventricular Mass Index [Derived from Ikonomidis et al.](5) 


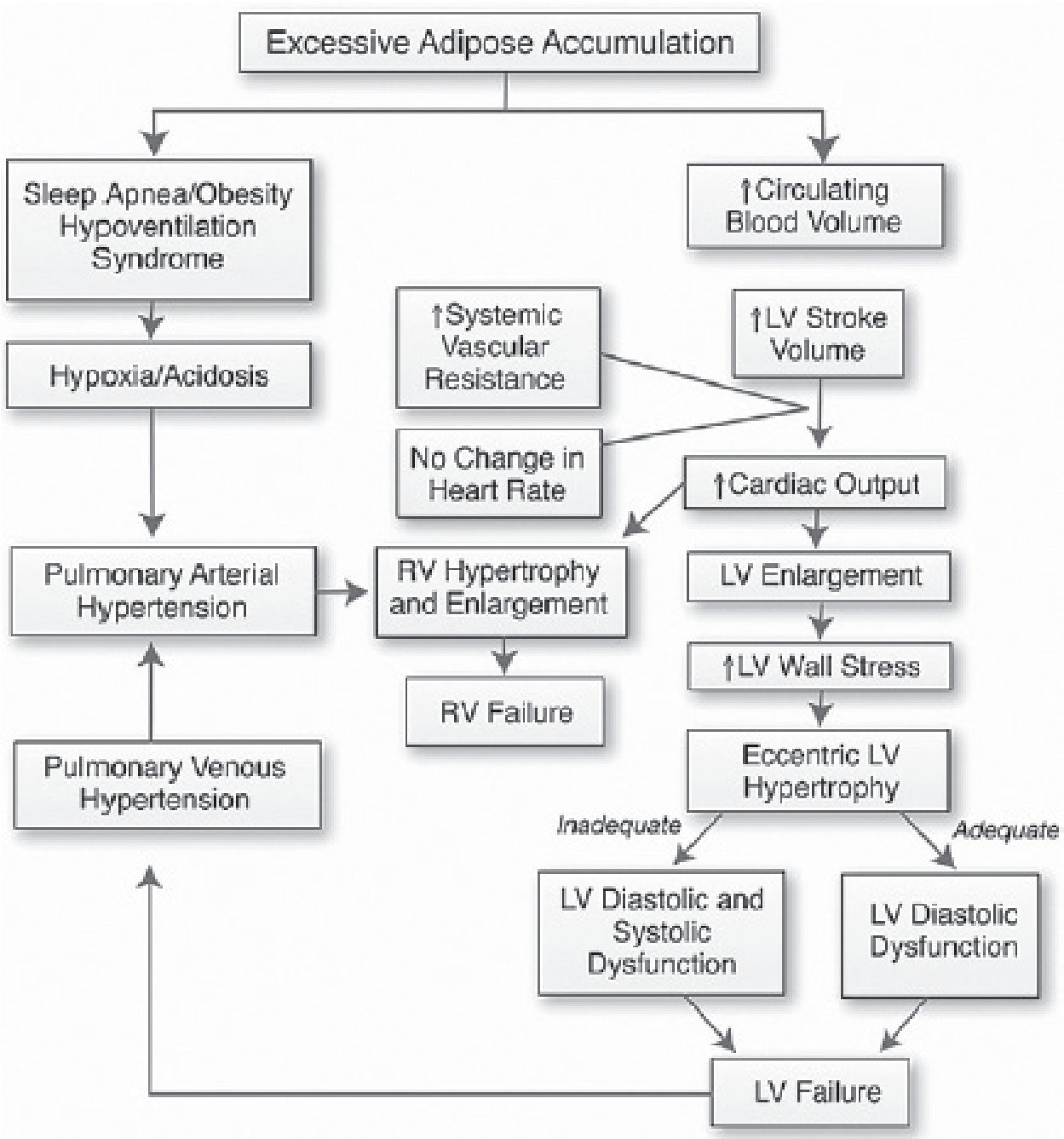

FIGURE 2.2: Pathophysiological changes induced by obesity on the cardiac and hemodynamic function (Derived from Lavie et al. (59) and Adams et al. (60)) 


\subsubsection{SYSTOLIC AND DIASTOLIC FUNCTION}

Most people with obesity have normal to normal-high hemodynamic function, in terms of heart rate and blood pressure.(6, 7, 11-14) However, obese often suffer hypertension compared to lean, frequently as part of the metabolic syndrome. Also, a significant greater stroke volume and cardiac output $(6,7)$ (or an increasing trend (10)) is observed.

Systolic function is often similar between obese and lean. $(11,15,16)$ No significant difference, or only a decreasing trend, was found in LV ejection fraction (EF) and LV fractional shortening (FS) (one-dimensional surrogate of ejection fraction).(4, 6, 7, 10, 17)

Diastolic dysfunction is quite common in obese, $(4,7,12,15)$ which is probably a consequence than a cause given the higher incidence of $\mathrm{LVH}$ in obese. The ratio of the mitral peak velocity of early filling to late filling (E/A-ratio) is <1 in $17-52.9 \%$ of the obese, $(11,13,16$, 17) and significant more reduced compared to lean.(6) Also, increased deceleration time and isovolumetric relaxation time are found in obese,(8) as well as increased LA dimensions. (6, 8) All of them are seen in diastolic dysfunction.

\subsubsection{CHRONIC HEART FAILURE}

The mean duration of obesity is a significant factor for left and/or right ventricular failure. (18) No direct relationship was found in mean body mass index (BMI), heart rate, and systolic and diastolic blood pressures between obese with and without chronic heart failure. (18)

\subsection{AFTER BARIATRIC SURGERY: THE "NOT SO" OBESE}

\subsubsection{WEIGHT LOSS}

Most people with obesity develop significant weight loss after bariatric surgery, however, they remain clinically obese with a BMI around $29-34 \mathrm{~kg} / \mathrm{m} 2$ after 3-36 months and even after 10 years of surgery. $(4,7,8,10-17,19-38)$ Most weight loss is seen in the first three months and declines in the months thereafter, with a smaller decrease in weight 6-36 months after surgery. $(8,12,15-17,19,24,27,30)$ In these first three months, the cardiac remodelling must occur.

\subsubsection{HEMODYNAMIC FUNCTION}

The effect of excessive weight loss on blood pressure varies widely. Several studies found a short and long term significant improvement in systolic and diastolic blood pressure, (13, $15-17,26,29,30,37)$ as well as a significant decrease in mean arterial blood pressure.(4, 
22) However other studies showed only a long term significant decrease in systolic blood pressure, $(8,14,21,36)$ while others have the opposite results; a significant decrease in diastolic blood pressure. $(10,25,27)$ About the same amount of studies found no short and long-term significant differences in systolic or diastolic blood pressure or only a decreasing trend. $(7,8,10-12,19,20,22,23,28,31,33)$ When a (significant) decrease is seen, it is often in obese with high blood pressure and/or LVM before surgery. $(20,35)$

A similar effect is seen on heart rate; Several studies found a significant decrease in heart rate 3-24 months, and even 3.6-10 years, after surgery,(6, 10-14, 22, 25-27, 36, 37) but just as much other studies did not found any significant changes in heart rate after 4-24 months, or only a decreasing trend. $(4,7,19,20,28,31-33,35)$ It seems that pre-existing hypertension or $\mathrm{LVH}$ is a prerequisite for reverse remodelling after surgery.

More effect of excessive weight loss in systolic function is seen in cardiac output and stroke volume. Cardiac output was the only systolic parameter, in all studies, which reported it, showed a significant decrease after 6-24 months and even after 10 years. $(6,7,10,13,14,22)$ A significant reduction, or a decreasing trend, in stroke volume was found after 3-24 months and even after 10 years (by decrease in $\operatorname{EDV}$ and $\operatorname{ESV})(6,7,10,22,31)$ however, one study with a decreasing trend after 3 months, showed a return to baseline after 17 months, while BMI continued to decline.(27) These changes indicate an improvement in volume overload, which can be the start of normalization of cardiac remodelling.

\subsubsection{CARDIAC GEOMETRY}

\section{Left ventricle}

Excessive weight loss can lead to a significant reduction in LV dimensions after 3-36 months and even after 10 years. $(7,8,10,15,16,22,23,32)$ Sometimes, a significant improvement is only seen in obese with LVH before surgery, $(4,20,35)$ and a decreasing, but not significant, trend in obese without LVH before surgery.(20) However, it does not always lead to significant changes, or a decreasing trend after 3-24 months. $(4,6,13,14,21,26,28,29,31$, $33,37)$ Some studies even found an increase in LV end-diastolic or end-systolic dimension after 6-36 months. $(17,27)$

In almost every study, LVM, LVM/height2.7 or LVM/BSA decreases significantly after 3-36 months and even after 3.6-10 years of excessive weight loss.(6-8, 10, 11, 14-17, 19, 21-27, 29, $30,32,36,37)$ It continues to decrease linear up to $24-36$ months, while most other variables, such as weight loss, plateaus after several months. $(12,13,17,19,28)$ One study only showed a significant reduction in LVM in obese with LVH before surgery, but not in people without LVH.(35) A significant decrease was not always found in LVM/BSA after 3-36 months, (7, 8, 
$17,27,31)$ which is probably the result of a proportional decrease in LVM and body surface area after excessive weight loss. Also, no change in LVM/end-diastolic volume ratio is seen and remained higher than in lean people after 12 months.(7) In addition to the decrease in LVM, a significant reduction is also seen in IVST and PWT after 3-36 months and even after 3.6 years of excessive weight loss, $(8,15,16,22,24-26,28,36)$ as well as in RWT. $(6,13,14$, $17,21,26,29)$ However, not all studies found significant decrease in these parameters after 3-36 months, $(12,17,23,37)$ or only in obese with LVH, but not in obese without LVH before surgery. (35) Improvement in LV geometry is observed after 3-36 months of excessive weight loss. $(13,16,17,21,26)$

One study showed a complete normalization in geometry in $36.6 \%$ of the obese, $51.2 \%$ changed into an improved pattern, 39\% showed no changes and 9.8\% became worse, after 12 months.(13) However, another group found no mean significant changes in the prevalence of LVH in obese 12 months after surgery; A normalized geometry was seen in $9.6 \%$, though 12.0\% developed a new LV geometry anomaly.(23)

\section{Right ventricle}

Excessive weight loss causes a significantly decrease in RV mass after 3-24 months. $(7,14,27)$ RV dimensions are often not improved after 3-17 months, $(15,25,27,37)$ however, one study found a significant decrease in RV end-diastolic volume, but not in RV end-systolic volume after 12 months.(7)

The RV systolic pressure results vary between no significant changes after 7.6 months,(37) a significant decrease after 22.7 months(31) and a decreasing trend after 3.6 years(25) (Measured with Doppler imaging). The RV ejection fraction and volumes remained unchanged after 3-17 months. $(7,27)$

Significant changes were found in RV fractional area,(14) RV isovolumetric contraction time, isovolumetric relaxation, ejection time,(33) and RV myocardial performance index after 12-24 months of excessive weight loss. $(25,33)$ Also, peak systolic and early diastolic tricuspid annular velocities significantly increased, as well as the ratio of early-to-late diastolic velocities at the tricuspid annulus.(37) All indicators of a better RV function.

\subsubsection{SYSTOLIC AND DIASTOLIC FUNCTION}

Systolic function was quite similar in obese compared to lean before surgery, and remained this way after 3-36 months of excessive weight loss. (7, 11-14, 17, 26, 28, 31, 32, 37) However, some studies did find a significant increase in LVEF after 6-12 months up to 3.6 years of 
excessive weight loss, $(16,25,30,36)$ or only a significant increase in LVFS in obese with low pre-operative LVFS after 4.3 months of surgery.(4)

In addition to the improvements in cardiac output and stroke volume, diastolic function improved significant, or showing a trend, in most studies as well, after 3-24 months up to 3.610 years of excessive weight loss. $(6,8,10,13,15,16,22,26,28,32,36,37,39)$ A significant increased E/A ratio is was frequently found, $(6,10,13,15,16,22,26,28,36,37,39)$ even when there was no significant changes in peak E and A velocity, $(28,39)$ or only in one of them. $(6,13,15,36)$ However, one study did not found a significant change after 22.7 months. (31) Improvements are also seen in LV isovolumetric relaxation time, $(6,8,10,17,22,26,28)$ deceleration time, $(8,10,12,13,31)$ and left atrial dimensions $(6,10,13,16,21,22,26,30,32)$ after 3-36 months and 3.6-10 years. However, improvements in left atrial dimensions are not always observed, $(15,37)$ some only found a decreasing trend.(14) Also, in a couple of studies, the deceleration time remained the same. $(15,37)$

\subsubsection{CHRONIC HEART FAILURE}

Improvements in CHF symptoms and Assessment of New York Heart Association functional class were observed 6-12 months after surgery, $(18,34,38)$ indicating that excessive weight loss has a positive effect on cardiac remodelling. These findings are supported by several case series investigating the effect of bariatric surgery on LVEF in patients with end-stage heart failure and/or patients scheduled for heart transplantation. $(34,38,40-42)$

No difference is found between the amounts of weight loss after 4.5 months between obese with or without CHF, as well as in hemodynamic, systolic and diastolic function. The only significant difference was found in right ventricular internal dimension. All other parameters improved in the same amount as in obese without CHF.(18)

Obese with CHF showed a significant increase in LVEF from an average of 23\% to $32 \%$ after 6 months and an average of $21.7 \%$ to $35.0 \%$ after 12 months of excessive weight loss.(34, 38) Also a trend towards normalization in LV size is seen after 12 months.(38) The effect on LV diastolic diameter and left atrial size was a non-significant improving trend after 12 months.(38) Also, no significant changes were found in heart rate, systolic and diastolic blood pressure.(38) 


\subsection{IMPLICATIONS FOR CLINICAL PRACTICE}

In summary, bariatric surgery has beneficial effects on systolic and diastolic function and myocardial structure. (9) Pre-existing hypertension or LVH seems to be a prerequisite for reverse remodelling after bariatric surgery. But there are still questions that remain unanswered:

1) What are the possible mechanisms of these cardiac/hemodynamic function improvements?

2) Should cardiac pathology and obesity be an indication for a surgical procedure? And if so, which bariatric surgical procedure shows the most improvements?

3) Should anaesthesiological care be modified, with special attention for hemodynamic alterations, for patients who had bariatric surgery in the past?

\section{Physiology of the enterocardiac axis}

The findings of cardiovascular changes after bariatric surgery are noticeable (9) and may represent a supra-physiological effect of metabolic/bariatric surgical procedures. (43) Basically, the bariatric cardiac effects are considered to be metabolic and hemodynamic, rather than solely hemodynamic. (44) The classical hemodynamic weight-dependent effect of bariatric surgery is no longer thought to be the only one responsible for reverse remodelling seen after bariatric surgery, as the beneficial effects can be independent from changes in blood pressure. $(25,45)$ This includes that there is a possibility of direct gut hormonal inotropic action on the myocardium through an enterocardiac axis. $(9,43)$

\section{Cardiac pathology as indication for bariatric surgery}

Strictly taken, according to the guidelines of the International Federation of Surgery for Obesity and Metabolic disorders (IFSO) (46) cardiac pathology solely is not an indication for bariatric surgery. In patients with (end-stage) heart failure, several case series have demonstrated the positive effect of weight loss (due to bariatric surgery) on LVEF and the NYHA functional class (of heart failure). (34, 38, 40-42) Ristow et al. (47) reported on two patients who no longer required heart transplantation after successful weight reduction and improvement of LVEF.

Nowadays there is a shift going from bariatric surgery to metabolic surgery. $(2,48)$ In our opinion, there is enough evidence to state that obesity increases the risk of getting cardiac pathology, but nevertheless the current IFSO do not state them as comorbidity. 
Also there is increasing evidence that the metabolic profile of obese is associated with changes in cardiovascular parameters $(4,18,20,35,49-53)$ and therefore we think that the current IFSO criteria need to be adjusted incorporating a more 'metabolic approach.' Currently there is no consensus on which bariatric surgical procedures has the most benefits, in terms of cardiovascular hemodynamics.

\section{Modifications of perioperative care for ex-bariatric patients?}

As summarised in the recent systematic review by Aggarwal et al. (9) bariatric surgery has beneficial short term effects on cardiovascular physiology in general. Most of the preoperative abnormal cardiac parameters normalise over the course of bariatric surgery. So from an anaesthesiological point of view, ex-bariatric patients might not have to be treated differently than other patients in future (anaesthesiological) care concerning cardiac hemodynamics. However, it is uncertain whether ex-bariatric patients need to be screened before any future surgical procedure, because there is a wide variety in cardiovascular physiological changes between the different ex-bariatric patients. (9) Also, most cardiovascular changes are seen in obese with the greatest abnormalities before bariatric surgery. (4, 18, 20, 35, 49-53) Even that they now can have normal cardiovascular physiology, it is important to realise that their cardiovascular system has had much to endure for years, which might make them less responsive in alterations during surgery than patients who have never been obese. As mentioned by Alpert et al. (18) the duration of obesity is significantly associated with the severity of possible heart conditions. In the light of this we introduce the term fat-years in comparison with the term pack years in smoking. End organ (cardiac) dysfunction is increased by severity in combination with duration of obesity. So anaesthesiologists should be aware of this and be careful with the older ( $>60$ years of age) bariatric or obese patients. Admission to an advanced care unit might be necessary for this group. (54) In the current body of literature, there is extensive knowledge about the preoperative characteristics and aspects concerning patients with a history of heart disease $(s)(55,56)$, but less is known about ex-bariatric patients and the changes in cardiovascular hemodynamics and the possible implications for future surgery and anaesthesia. Also the literature is sparse about the long-term changes in cardiovascular parameters and therefore it is difficult to determine (based on the current literature) clinical implications. Long-term studies are needed to investigate cardiovascular changes as a result of surgical weight loss. 


\subsection{RECOMMENDATIONS}

Based on current literature several recommendations can be made regarding the cardiac remodelling after bariatric surgery and its implications for clinical practice. As stated earlier in this review there is an extensive body of literature that shows that weight loss and in particular bariatric surgery has beneficial effects in terms of improvement of cardiac and hemodynamic function. In our opinion, every patient with a medical history that includes cardiac pathology and obesity, should be considered for bariatric surgery. The presence of hypertension or LVH seems to be a prerequisite for reverse remodelling after bariatric surgery. However, it is unclear which bariatric surgical procedure is the best choice to induce the aforementioned cardiac function and hemodynamic changes. Most of the current research is done in patients who had either a laparoscopic gastric bypass or laparoscopic gastric sleeve. (9) Studies investigating the effects of the newer bariatric surgical procedures on cardiac function are sparse and in some cases not even available yet (for instance the effects of the diverted sleeve gastrectomy with either an ileal interposition of transit bipartition $(57,58)$ ) This also included the effects of enteric hormones on cardiac and hemodynamic function (25, 45 ) that might indicate a possible physiological inotropic (inter)action on the myocardium through an enterocardiac axis. $(9,43)$

Second issue that arises is that we currently do not have insight in the exact long-term effects of bariatric surgery on cardiac function. Most of the studies have a short follow-up length, so to give a recommendation regarding the long-term effects is simply not possible. Therefore we desperately need large randomised controlled trials to investigate these effects.

\subsection{CONCLUSION}

Bariatric surgery offers beneficial cardiac effects. These effects might be the result of a combined hemodynamic and metabolic effect of surgical modulation of the enterocardiac axis. Essential for reverse cardiac remodelling seems to be age, the pre-existence of hypertension and left ventricular hypertrophy. The current IFSO guidelines regarding indications for bariatric surgery need to be changed with great urgency, because bariatric surgery is essential for cardiac remodelling in the obese, and to improve their (cardiac) disease.

Future studies must focus on identifying the most successful bariatric surgical procedure in preventing and treating obesity-related heart disease. Also the long-term changes in cardiovascular parameters after surgery and implications for future anaesthesia and surgery are directions for future research. 


\subsection{CARDIAC REMODELLING DEFINITIONS}

To describe the cardiovascular physiology and cardiac remodelling in obese before and after bariatric surgery, specific measurements are used. Systolic function represents the contractility of the ventricle and can be described in terms of ejection fraction, fractional shortening, stroke volume and cardiac output. $(5,61)$ Diastolic function represents the filling ability of the ventricle and can be described in terms of E/A-ratio, left atrial dimensions, deceleration time and isovolumetric relaxation time. (5), (61)

\section{General:}

- $\quad$ BSA = body surface area

- $\quad \mathrm{CO}=$ cardiac output; blood volume pumped out of the ventricle per time period, calculated by stroke volume $\mathrm{x}$ heart rate (SV x HR)

- $\quad$ DT = deceleration time of early diastolic filling (E); the time peak velocity of early diastolic filling (E) declines in early diastole

- $\quad$ E/A-ratio = ratio of peak velocity of early diastolic filling (E) to peak (atrial) velocity of late diastolic filling (A)

- $\quad \mathrm{EDD}=$ end-diastolic dimension; diameter across the right or left ventricle at the end of diastole (5)

- $\quad \mathrm{EDV}=$ end-diastolic volume; the volume of the right or left ventricle at the end of diastole (5)

- $\quad E F=$ ejection fraction; percentage of blood volume that is pumped out of the right or left ventricle with each cardiac contraction, calculated by ((EDV-ESV)/EDV) x 100\% or $(\mathrm{SV} / \mathrm{EDV}) \times 100 \%$.

- $\quad \mathrm{ESD}=$ end-systolic dimension; diameter across the right or left ventricle at the end of systole (5)

- $\quad \mathrm{ESV}=$ end-systolic volume; the volume of the right or left ventricle at the end of systole (5)

- $\quad$ FAC = fractional area change; percentage in change between the area from end diastole to end systole, calculated by ((end-diastolic area - end-systolic area)/end-diastolic area) x $100 \%$

- $\quad F S=$ fractional shortening; percentage of change in right or left ventricle dimension between end-diastole and end-systole dimension, calculated by ((EDD-ESD)/EDD) $\mathrm{x}$ $100 \%(61)$

- $\quad H R=$ heart rate; the number of cardiac contraction during a certain time period (often per minute) 
- $\quad$ IVCT = isovolumetric contraction time; time between the end of diastole and the opening of the semilunar valves, when the pressure in the ventricles rapidly increases while blood volume remains constant (5)

- $\quad$ IVRT = isovolumetric relaxation time; time between the end of systolic ejection and the opening of the atrioventricular valves, when the pressure in the ventricles rapidly decreases while blood volume remains constant (5)

- $\quad$ IVSd = interventricular septum dimension; diameter of the ventricular septum at enddiastolic (61)

\section{Left ventricle:}

- $\quad$ LAD = left atrial dimension; diameter across the left atrium at the end-ventricular systole (61)

- $\quad$ LAV = left atrial volume; volume of the left atrium at the end-ventricular systole (61)

- $\quad$ LVEDD = left ventricular end-diastolic dimension; diameter across the left ventricle at the end of diastole (5)

- $\quad$ LVEDV = left ventricular end-diastolic volume; the volume of the left ventricle at the end of diastole (5)

- $\quad$ LVESD = left ventricular end-systolic dimension; diameter across the left ventricle at the end of systole (5)

- $\quad$ LVESV = left ventricular end-systolic volume; the volume of the left ventricle at the end of systole (5)

- LV geometry = left ventricular configuration patterns; the LV can be classified in four different types based on LVM and RWT: eccentric hypertrophy (increased LVM and normal RWT), concentric hypertrophy (increased LVM and RWT), concentric remodelling (normal LVM and increased RWT) and normal geometry (normal LVM and RWT) (61)

- $\quad$ LVH = left ventricular hypertrophy; defined as an increased LVM due to an increase in LV dimension or wall thickness or both at end-diastole. For classification of LVH in concentric or eccentric RWT is used (61)

- $\quad$ LVM = left ventricular mass; is estimated by LV dimension and LV wall thickness (IVSd and PWd) at end-diastole and is used to define LVH. (61)LVM can also be described as LVM/height2, LVM/height2.7, LVM/lean body mass, LVM/BSA or LVM/LVEDV to improve comparison between subjects (5)

- $\quad$ PWd = posterior wall dimension; diameter of the posterior wall of the LV at enddiastolic (5) 


\section{Right ventricle:}

- $\quad$ RVEDD = right ventricular end-diastolic dimension; diameter across the right ventricle at the end of diastole (5)

- $\quad$ RVEDV = right ventricular end-diastolic volume; the volume of the right ventricle at the end of diastole (5)

- $\quad$ RVESD = right ventricular end-systolic dimension; diameter across the right ventricle at the end of systole (5)

- $\quad$ RVESV = right ventricular end-systolic volume; the volume of the right ventricle at the end of systole (5)

- $\quad$ RVET = right ventricular ejection time; duration for the blood to be pumped out of the right ventricle during contraction. This measurement is used for the calculation of the RVMP (61)

- $\quad$ RVMPI = right ventricular myocardial performance index (also called Tei index); index measurement for global systolic and diastolic ventricular function, calculated by (IVCT+IVRT)/RVET or (TCOT-RVET)/RVET (61)

- $\quad$ RWT = relative wall thickness; calculated by $(2 \times \mathrm{PWd}) / \mathrm{LVEDD}$ and is used to distinguish, concentric and eccentric LVH (61)

- $\quad \mathrm{SV}=$ stroke volume; volume of blood pumped out of the right or left ventricle with each cardiac contraction, calculated by EDV-ESV or estimated by echocardiography.

- $\quad$ TCOT $=$ tricuspid valve closure to opening time; time between closing and opening of the tricuspid valve. This measurement is used for the calculation of the RVMPI (61). 


\subsection{REFERENCES}

1. Ng M, Fleming T, Robinson M, Thomson B, Graetz N, Margono C, et al. Global, regional, and national prevalence of overweight and obesity in children and adults during 1980-2013: a systematic analysis for the Global Burden of Disease Study 2013. Lancet (London, England). 2014;384(9945):766-81.

2. Buchwald H, Avidor Y, Braunwald E, Jensen MD, Pories W, Fahrbach K, et al. Bariatric surgery: a systematic review and meta-analysis. Jama. 2004;292(14):1724-37.

3. Alexander JK. The cardiomyopathy of obesity. Prog Cardiovasc Dis. 1985;27(5):325-34.

4. Alpert MA, Terry BE, Kelly DL. Effect of weight loss on cardiac chamber size, wall thickness and left ventricular function in morbid obesity. The American journal of cardiology. 1985;55(6):783-6.

5. Lang RM. Recommendations for cardiac chamber quantification by echocardiography in adults: an update from the American Society of Echocardiography and the European Association of Cardiovascular Imaging. Eur Heart J Cardiovasc Imaging. 2015;16(2):233-70.

6. Karason K, Wallentin I, Larsson B, Sjostrom L. Effects of obesity and weight loss on cardiac function and valvular performance. Obesity research. 1998;6(6):422-9.

7. Rider OJ, Francis JM, Ali MK, Petersen SE, Robinson M, Robson MD, et al. Beneficial cardiovascular effects of bariatric surgical and dietary weight loss in obesity. Journal of the American College of Cardiology. 2009;54(8):718-26.

8. Ikonomidis I, Mazarakis A, Papadopoulos C, Patsouras N, Kalfarentzos F, Lekakis J, et al. Weight loss after bariatric surgery improves aortic elastic properties and left ventricular function in individuals with morbid obesity: a 3-year follow-up study. Journal of hypertension. 2007;25(2):43947 .

9. Aggarwal R, Harling L, Efthimiou E, Darzi A, Athanasiou T, Ashrafian H. The Effects of Bariatric Surgery on Cardiac Structure and Function: a Systematic Review of Cardiac Imaging Outcomes. Obesity surgery. 2015.

10. Kardassis D, Bech-Hanssen O, Schonander M, Sjostrom L, Petzold M, Karason K. Impact of body composition, fat distribution and sustained weight loss on cardiac function in obesity. International journal of cardiology. 2012;159(2):128-33.

11. Leichman JG, Aguilar D, King TM, Mehta S, Majka C, Scarborough T, et al. Improvements in systemic metabolism, anthropometrics, and left ventricular geometry 3 months after bariatric surgery. Surgery for obesity and related diseases : official journal of the American Society for Bariatric Surgery. 2006;2(6):592-9.

12. Leichman JG, Wilson EB, Scarborough T, Aguilar D, Miller CC, 3rd, Yu S, et al. Dramatic reversal of derangements in muscle metabolism and left ventricular function after bariatric surgery. The American journal of medicine. 2008;121(11):966-73.

13. Luaces M, Cachofeiro V, Garcia-Munoz-Najar A, Medina M, Gonzalez N, Cancer E, et al. Anatomical and functional alterations of the heart in morbid obesity. Changes after bariatric surgery. Revista espanola de cardiologia (English ed). 2012;65(1):14-21. 
14. Owan T, Avelar E, Morley K, Jiji R, Hall N, Krezowski J, et al. Favorable changes in cardiac geometry and function following gastric bypass surgery: 2-year follow-up in the Utah obesity study. Journal of the American College of Cardiology. 2011;57(6):732-9.

15. Martin J, Bergeron S, Pibarot P, Bastien M, Biertho L, Lescelleur O, et al. Impact of bariatric surgery on $\mathrm{N}$-terminal fragment of the prohormone brain natriuretic peptide and left ventricular diastolic function. The Canadian journal of cardiology. 2013;29(8):969-75.

16. Iancu ME, Copaescu C, Serban M, Ginghina C. Favorable changes in arterial elasticity, left ventricular mass, and diastolic function after significant weight loss following laparoscopic sleeve gastrectomy in obese individuals. Obesity surgery. 2014;24(3):364-70.

17. Cunha Lde C, da Cunha CL, de Souza AM, Chiminacio Neto N, Pereira RS, Suplicy HL. Evolutive echocardiographic study of the structural and functional heart alterations in obese individuals after bariatric surgery. Arquivos brasileiros de cardiologia. 2006;87(5):615-22.

18. Alpert MA, Terry BE, Mulekar M, Cohen MV, Massey CV, Fan TM, et al. Cardiac morphology and left ventricular function in normotensive morbidly obese patients with and without congestive heart failure, and effect of weight loss. The American journal of cardiology. 1997;80(6):736-40.

19. Algahim MF, Lux TR, Leichman JG, Boyer AF, Miller CC, 3rd, Laing ST, et al. Progressive regression of left ventricular hypertrophy two years after bariatric surgery. The American journal of medicine. 2010;123(6):549-55.

20. Alpert MA, Lambert CR, Terry BE, Cohen MV, Mulekar M, Massey CV, et al. Effect of weight loss on left ventricular diastolic filling in morbid obesity. The American journal of cardiology. 1995;76(16):1198-201.

21. Cavarretta E, Casella G, Cali B, Dammaro C, Biondi-Zoccai G, Iossa A, et al. Cardiac remodeling in obese patients after laparoscopic sleeve gastrectomy. World journal of surgery. 2013;37(3):56572.

22. Di Bello V, Santini F, Di Cori A, Pucci A, Talini E, Palagi C, et al. Effects of bariatric surgery on early myocardial alterations in adult severely obese subjects. Cardiology. 2008;109(4):241-8.

23. Dzenkeviciute V, Petrulioniene Z, Sapoka V, Aidietiene S, Abaraviciute L. The effect of weight loss on the cardiac structure and function after laparoscopic adjustable gastric banding surgery in morbidly obese individuals. Obesity surgery. 2014;24(11):1961-8.

24. Gahtan V, Goode SE, Kurto HZ, Schocken DD, Powers P, Rosemurgy AS. Body composition and source of weight loss after bariatric surgery. Obesity surgery. 1997;7(3):184-8.

25. Garza CA, Pellikka PA, Somers VK, Sarr MG, Collazo-Clavell ML, Korenfeld Y, et al. Structural and functional changes in left and right ventricles after major weight loss following bariatric surgery for morbid obesity. The American journal of cardiology. 2010;105(4):550-6.

26. Hsuan CF, Huang CK, Lin JW, Lin LC, Lee TL, Tai CM, et al. The effect of surgical weight reduction on left ventricular structure and function in severe obesity. Obesity (Silver Spring, Md). 2010;18(6):1188-93.

27. Jhaveri RR, Pond KK, Hauser TH, Kissinger KV, Goepfert L, Schneider B, et al. Cardiac remodeling after substantial weight loss: a prospective cardiac magnetic resonance study after bariatric surgery. Surgery for obesity and related diseases : official journal of the American Society for Bariatric Surgery. 2009;5(6):648-52. 
28. Kanoupakis E, Michaloudis D, Fraidakis O, Parthenakis F, Vardas P, Melissas J. Left ventricular function and cardiopulmonary performance following surgical treatment of morbid obesity. Obesity surgery. 2001;11(5):552-8.

29. Karason K, Wallentin I, Larsson B, Sjostrom L. Effects of obesity and weight loss on left ventricular mass and relative wall thickness: survey and intervention study. BMJ (Clinical research ed). 1997;315(7113):912-6.

30. Kokkinos A, Alexiadou K, Liaskos C, Argyrakopoulou G, Balla I, Tentolouris N, et al. Improvement in cardiovascular indices after Roux-en-Y gastric bypass or sleeve gastrectomy for morbid obesity. Obesity surgery. 2013;23(1):31-8.

31. Koshino Y, Villarraga HR, Somers VK, Miranda WR, Garza CA, Hsiao JF, et al. Changes in myocardial mechanics in patients with obesity following major weight loss after bariatric surgery. Obesity (Silver Spring, Md). 2013;21(6):1111-8.

32. Lin CH, Kurup S, Herrero P, Schechtman KB, Eagon JC, Klein S, et al. Myocardial oxygen consumption change predicts left ventricular relaxation improvement in obese humans after weight loss. Obesity (Silver Spring, Md). 2011;19(9):1804-12.

33. Maniscalco M, Arciello A, Zedda A, Faraone S, Verde R, Giardiello C, et al. Right ventricular performance in severe obesity. Effect of weight loss. European journal of clinical investigation. 2007;37(4):270-5.

34. McCloskey CA, Ramani GV, Mathier MA, Schauer PR, Eid GM, Mattar SG, et al. Bariatric surgery improves cardiac function in morbidly obese patients with severe cardiomyopathy. Surgery for obesity and related diseases : official journal of the American Society for Bariatric Surgery. 2007;3(5):503-7.

35. Mukerji R, Petruc M, Fresen JL, Terry BE, Govindarajan G, Alpert MA. Effect of weight loss after bariatric surgery on left ventricular mass and ventricular repolarization in normotensive morbidly obese patients. The American journal of cardiology. 2012;110(3):415-9.

36. Valezi AC, Machado VH. Morphofunctional evaluation of the heart of obese patients before and after bariatric surgery. Obesity surgery. 2011;21(11):1693-7.

37. Willens HJ, Chakko SC, Byers P, Chirinos JA, Labrador E, Castrillon JC, et al. Effects of weight loss after gastric bypass on right and left ventricular function assessed by tissue Doppler imaging. The American journal of cardiology. 2005;95(12):1521-4.

38. Ramani GV, McCloskey C, Ramanathan RC, Mathier MA. Safety and efficacy of bariatric surgery in morbidly obese patients with severe systolic heart failure. Clinical cardiology. 2008;31(11):51620.

39. Garza CA, Pellikka PA, Somers VK, Sarr MG, Seward JB, Collazo-Clavell ML, et al. Major weight loss prevents long-term left atrial enlargement in patients with morbid and extreme obesity. European journal of echocardiography : the journal of the Working Group on Echocardiography of the European Society of Cardiology. 2008;9(5):587-93.

40. Samaras K, Connolly SM, Lord RV, Macdonald P, Hayward CS. Take heart: bariatric surgery in obese patients with severe heart failure. Two case reports. Heart, lung \& circulation. 2012;21(12):847-9. 
41. Lim CP, Fisher OM, Falkenback D, Boyd D, Hayward CS, Keogh A, et al. Bariatric Surgery Provides a "Bridge to Transplant" for Morbidly Obese Patients with Advanced Heart Failure and May Obviate the Need for Transplantation. Obesity surgery. 2015.

42. Chaudhry UI, Kanji A, Sai-Sudhakar CB, Higgins RS, Needleman BJ. Laparoscopic sleeve gastrectomy in morbidly obese patients with end-stage heart failure and left ventricular assist device: medium-term results. Surgery for obesity and related diseases : official journal of the American Society for Bariatric Surgery. 2015;11(1):88-93.

43. Ashrafian H, le Roux CW, Darzi A, Athanasiou T. Effects of bariatric surgery on cardiovascular function. Circulation. 2008;118(20):2091-102.

44. Ashrafian H, Athanasiou T, le Roux CW. Heart remodelling and obesity: the complexities and variation of cardiac geometry. Heart (British Cardiac Society). 2011;97(3):171-2.

45. Vest AR, Heneghan HM, Agarwal S, Schauer PR, Young JB. Bariatric surgery and cardiovascular outcomes: a systematic review. Heart (British Cardiac Society). 2012;98(24):1763-77.

46. Fried M, Yumuk V, Oppert JM, Scopinaro N, Torres A, Weiner R, et al. Interdisciplinary European guidelines on metabolic and bariatric surgery. Obesity surgery. 2014;24(1):42-55.

47. Ristow B, Rabkin J, Haeusslein E. Improvement in dilated cardiomyopathy after bariatric surgery. Journal of cardiac failure. 2008;14(3):198-202.

48. Buchwald H. The evolution of metabolic/bariatric surgery. Obesity surgery. 2014;24(8):1126-35.

49. Alpert MA, Lambert CR, Panayiotou H, Terry BE, Cohen MV, Massey CV, et al. Relation of duration of morbid obesity to left ventricular mass, systolic function, and diastolic filling, and effect of weight loss. The American journal of cardiology. 1995;76(16):1194-7.

50. Alpert MA, Lambert CR, Terry BE, Kelly DL, Panayiotou H, Mukerji V, et al. Effect of weight loss on left ventricular mass in nonhypertensive morbidly obese patients. The American journal of cardiology. 1994;73(12):918-21.

51. Alpert MA, Nusair MB, Mukerji R, Omran J, Mehra A, Ardhanari S, et al. Effect of weight loss on ventricular repolarization in normotensive severely obese patients with and without heart failure. The American journal of the medical sciences. 2015;349(1):17-23.

52. Alpert MA, Terry BE, Hamm CR, Fan TM, Cohen MV, Massey CV, et al. Effect of weight loss on the ECG of normotensive morbidly obese patients. Chest. 2001;119(2):507-10.

53. Alpert MA, Terry BE, Lambert CR, Kelly DL, Panayiotou H, Mukerji V, et al. Factors influencing left ventricular systolic function in nonhypertensive morbidly obese patients, and effect of weight loss induced by gastroplasty. The American journal of cardiology. 1993;71(8):733-7.

54. Buise MP, van den Broek RJC, de Zoete JP, Bindels AJGH. Advanced Care admission following bariatric surgery. Netherlands Journal of Critical Care. 2010;14(2):85-91.

55. Chand B, Gugliotti D, Schauer P, Steckner K. Perioperative management of the bariatric surgery patient: focus on cardiac and anesthesia considerations. Cleveland Clinic journal of medicine. 2006;73 Suppl 1:S51-6.

56. Katkhouda N, Mason RJ, Wu B, Takla FS, Keenan RM, Zehetner J. Evaluation and treatment of patients with cardiac disease undergoing bariatric surgery. Surgery for obesity and related diseases : official journal of the American Society for Bariatric Surgery. 2012;8(5):634-40. 
57. Celik A, Ugale S. Functional restriction and a new balance between proximal and distal gut: the tools of the real metabolic surgery. Obesity surgery. 2014;24(10):1742-3.

58. Celik A, Ugale S, Ofluoglu H, Vural E, Cagiltay E, Cat H, et al. Metabolic Outcomes of Laparoscopic Diverted Sleeve Gastrectomy with Ileal Transposition (DSIT) in Obese Type 2 Diabetic Patients. Obesity surgery. 2015;25(11):2018-22.

59. Lavie CJ, Milani RV, Ventura HO. Obesity and cardiovascular disease: risk factor, paradox, and impact of weight loss. Journal of the American College of Cardiology. 2009;53(21):1925-32.

60. Adams JP, Murphy PG. Obesity in anaesthesia and intensive care. British journal of anaesthesia. 2000;85(1):91-108.

61. Echocardiography CSO. Echocardiography http://csecho.ca/mdmath/2015 [cited 2015 08/10]. 


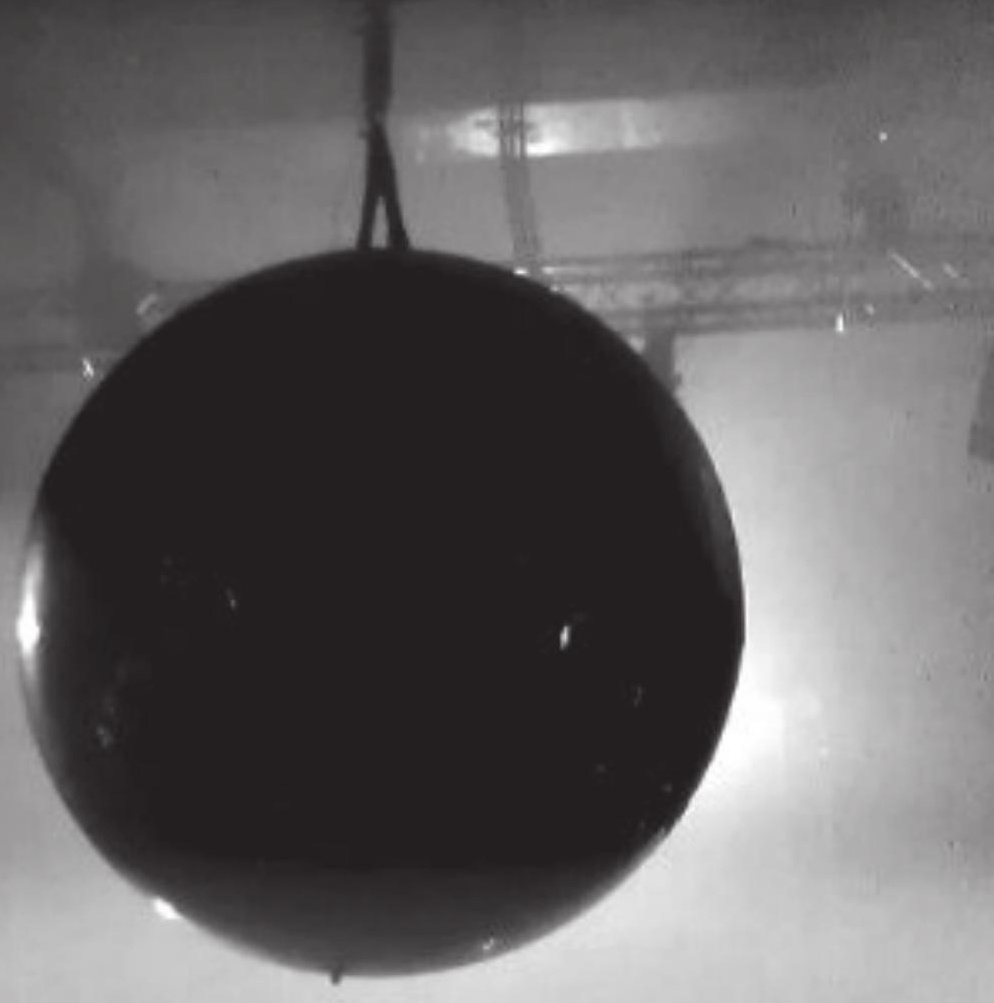

F 


\title{
3. VALIDATION OF THE NEXFIN ${ }^{\circledR}$ NON-INVASIVE CONTINUOUS BLOOD PRESSURE MONITORING VALIDATED AGAINST RIVA-ROCCI/KOROTKOFF IN A BARIATRIC PATIENT POPULATION
}

\author{
Sjaak Pouwels ${ }^{1,2}$ \\ Bianca Lascaris ${ }^{3}$ \\ Simon W. Nienhuijs ${ }^{3}$ \\ R. Arthur Bouwman ${ }^{3}$ \\ Marc P. Buise ${ }^{3}$
}

Accepted for publication in Journal of Clinical Anesthesia

1. Department of Surgery, Catharina Hospital, Eindhoven, The Netherlands

2. Department of Epidemiology, CAPHRI Research School, Maastricht University, The Netherlands

3. Department of Anaesthesiology, Intensive Care and Pain Medicine, Catharina Hospital, Eindhoven, The Netherlands 


\section{ABSTRACT}

\section{Background}

The present study aimed to validate the Nexfin ${ }^{\circledR}$ monitor and to assess the accuracy compared to classical sphyganomanometry (Riva-Rocci/Korotkoff (RRK)) blood pressure (BP) measurements in patients with obesity scheduled for bariatric surgery.

\section{Methods}

The validation process was done according to the protocols developed by the European Society of Hypertension from 2010. The Nexfin ${ }^{\circledast}$ monitor (Edwards Lifesciences / BMEYE B.V., Amsterdam, the Netherlands) calculates beat-to-beat blood pressure from finger pulse wave analysis. Measurements of systolic and diastolic BP were obtained using classical sphygmomanometry and the Nexfin ${ }^{\circledR}$ alternatingly. In total 33 patients were asked to participate in the validation process.

\section{Results}

In total 99 pairs of BP measurements were used. The device failed pass phase 1 as 65 systolic readings fell within $5 \mathrm{mmHg}$ (73 required). And 61, 76 and 90 diastolic readings fell within 5, 10 and $15 \mathrm{mmHg}$ respectively. Finally, it failed to pass phase 2 as 23 patients for systolic and 25 for diastolic had at least $2 / 3$ of their comparisons falling within $5 \mathrm{mmHg}$ ( 24 required) but 10 subjects for systolic and 8 for diastolic had all three comparisons more than $5 \mathrm{mmHg}$ different from the RRK readings (zero allowed). Mean differences were 7.8 $\pm 6.9 \mathrm{mmHg}$ for SBP and $8.0 \pm 7.2 \mathrm{mmHg}$ for DBP.

\section{Conclusion}

This study aimed to validate the Nexfin ${ }^{\circledR}$ monitor against Riva-Rocci/Korotkoff (RRK) blood pressure measurements in patients with obesity. Using the revised protocol of the European Society of Hypertension, the Nexfin ${ }^{\circledR}$ device was not able to pass validation. However using the original protocol, the Nexfin ${ }^{\circledast}$ device passed phase 1 and 2.1 of the validation process and failed to pass phase 2.2. Notwithstanding, these results, we think that the Nexfin ${ }^{\circledR}$ device is a reliable measurement tool to detect changes in cardiovascular hemodynamics. 


\subsection{INTRODUCTION}

Finger arterial pressure measurements can be used for non-invasive continuous blood pressure measurements. These methods use volume clamp methodology to reconstruct intraarterial pressures based on population- and individual patient characteristics. (1-3) Since the initial development of the volume-clamp methodology of Penaz (4) and the Physiocal criteria of Wesseling. (2), continuous blood pressure (BP) measurement became widely available and is used as substitute for intra-arterial BP measurements in both clinical and research settings. (5-7)Finger arterial pressure measurements, such as $\mathrm{Nexfin}^{\circledR}$, proof to be reliable under various conditions. These devices have been extensively validated for clinical practice and are being used in a variety of settings. [5-8] In 2009 Eeftinck Schattenkerk et al. (8) validated the Nexfin ${ }^{\circledast}$ monitor against Riva-Rocci/Korotkoff (RRK) BP measurement in healthy subjects. In a recent study by de Wilde et al. (9) the Nexfin ${ }^{\circledR}$ monitor was compared with radial arterial blood pressure in patients following major upper abdominal surgery. In terms of arterial blood pressure no significant differences were found between the radial arterial blood pressure and blood pressure measured with the Nexfin ${ }^{\circledast}$ monitor.

However, at the extreme ranges of patient characteristics, such as extreme/morbid obesity, general population characteristics may not apply and potential errors in blood pressure measurement may be introduced. The increasing prevalence of obesity makes validation of finger blood pressure measurements for this group clinical valuable, (10) because blood pressure cuff readings as well as intra-arterial line placement in (morbid) obese individuals can be challenging or even impossible. Fingers remain relatively thin in obesity, which may imply that finger blood pressure measurements using the volume clamp methodology may be feasible. The difficulty to assess blood pressure in morbid obese individuals makes an alternative technique very valuable.

The present study aimed to validate the Nexfin ${ }^{\circledR}$ monitor and to assess it's accuracy compared to classical sphyganomanometry (Riva-Rocci/Korotkoff (RRK)) BP measurements in patients with obesity scheduled for bariatric surgery. 


\subsection{METHODS}

\section{PATIENTS/ SUBJECTS}

Patients, who attended the Obesity Center in the Catharina Hospital in Eindhoven the Netherlands, were asked to take part in this validation study. Patients for revisional bariatric surgery, with hypertension, using antihypertensive drugs, with diabetes or renal disease were excluded. All measurements were performed within one session of 5 minutes per patient. This study was approved by the Medical Ethics Committee of the Catharina Hospital Eindhoven the Netherlands and all participants gave written informed consent.

\section{NON-INVASIVE CONTINUOUS BLOOD PRESSURE MEASUREMENT USING NEXFIN ${ }^{\circledR}$ HEMODYNAMIC MONITORING DEVICE}

The Nexfin ${ }^{\circledR}$ device uses the volume clamp method described by Penaz (3) and the Physiocal criteria of Wesseling (4), implemented in the TNO Finapres model 5 and Ohmeda Finapres 2300. Whereas the Nexfin uses the same principles to measure BP as TNO Finapres model 5 and Ohmeda Finapres 2300, the finger cuffs have been redesigned with a more efficient light emitting diode (LED) and photo-diode for a better signal-to-noise ratio. Secondly, the Nexfin ${ }^{\varpi}$ displays continuous pressures, which are based on reconstructed brachial wave shapes and levels. (11-14)

\section{RIVA-ROCCI/KOROTKOFF BLOOD PRESSURE MEASUREMENTS}

Riva-Rocci blood pressure measurements were used a reference method for validation. Since the mercury sphyganomanometry is no longer allowed in Dutch clinical practice, an automated cuff in- and deflation system with built in manometer was used (Boso Clinicus II, Henry Schein Medical, Almere, The Netherlands). This manometer shows an analogue representation of a mercury column and digital real time valves. Deflation rate was $2 \mathrm{mmHg} / \mathrm{s}$. The manometer was calibrated before the start of the study and checked after the conclusion of the study.

The validation team consisted of two persons (SP, BL). Both observers were trained in BP measurement according to the recommendation of the European Society of Hypertension. (15) Before the start of the study, the observers were instructed on how to use the Nexfin ${ }^{\circledast}$ device. Both the observers had no history of hearing problems and before the study started, five measurement sessions took place on volunteers, to accustom the observers to the procedure. A high-end stethoscope (Littmann, 3M Healthcare) with dual, clean and well fitting earpieces was used for RRK measurements. 


\section{MEASUREMENTS}

All measurements were done in a comfortable and quiet temperature controlled room. The participants were asked to relax for 10 minutes and any form of disturbance was prevented. Only two observers (SP and BL) were present. Each study participant was asked to sit in a comfortable chair and was asked neither to speak nor to move during the measurement. Appropriate cuff size was determined for both the finger and the arm in each participant, based on the circumference of the upper arm and the circumference of the midphalanx of the third digitus. An inflatable cuff with a length of $80 \%$ and a width at least $40 \%$ of the circumference of the upper arm deemed suitable according to Pickering et al. (16) The Nexfin ${ }^{\circledR}$ finger cuff was attached to the mid-phalanx of the third digit of the patients left hand; the manometric arm cuff was attached to the left upper arm. The heart reference system was placed on the middle of the left side of the thorax. The measured hand and the manometric arm cuff were placed at the mid-thorax to account for hydrostatic pressure differences. $(15,16)$

\section{PROCEDURE}

Two observers (SP and BL) were blinded from each other's and the measurements of the Nexfin ${ }^{\circledR}$ device and took five consecutive RRK BP measurements. The manometric arm cuff was inflated up to approximately $20 \mathrm{mmHg}$ above the systolic pressure and was automatically deflated at a rate of $2 \mathrm{mmHg} / \mathrm{s}$. All BP measurements were recorded to the nearest $2 \mathrm{mmHg}$ and respectively Korotkoff sound I and V were used. To eliminate possible inter-observer discrepancies in RRK measurements, a maximum difference of $4 \mathrm{mmHg}$ was accepted (both systolic and diastolic pressure).

After the complete deflation of the manometric arm cuff the Nexfin ${ }^{\circledR}$ signal was inaccurate for approximately 10 seconds due to post-occlusive hyperemic response which causes finger BP to swing. $(15,16)$ After a period of approximately 30 seconds the Nexfin ${ }^{\circledR}$ calculated the mean BP over a period of 30 seconds. After the Nexfin ${ }^{\circledR}$ measurement the next RRK measurement took place. In total nine sequential measurements took place between the Nexfin ${ }^{\circledR}$ device and the RRK measurements. An overview of these measurements is shown in table 3.1. Of these measurements the first two (the entry RRK measurement and Nexfin ${ }^{\circledR}$ ) were not included in the statistical analysis.

\section{STATISTICAL ANALYSIS}

This validation study followed the revised protocol developed by the European Society of Hypertension published in 2010. (17) Statistical Package for Social Sciences for Mac (SPSS, Chicago, IL, USA Version 20.0) was used to prepare the database and for statistical analysis. Continuous variables were presented as mean \pm standard deviation (SD). Categorical variables were presented as frequency with percentages. Data distribution was verified using 
the Shapiro-Wilk test. Values of $\mathrm{p}<0.05$ were considered statistically significant. Of BP1, BP3, BP5 and BP7 the mean of each pair of the observer measurements was calculated. The means were used to flank each Nexfin ${ }^{\circledast}$ measurement (BP2, BP4 and BP6). So BP2 was flanked by BP1 and BP3, BP4 by BP3 and BP5, and BP6 was flanked by BP5 and BP7. Differences were calculated between the observer measurements and the Nexfin ${ }^{\circledR}$ measurements. The difference was categorized into one of four categories (15); 0 - $5 \mathrm{mmHg}$ (measurements are very accurate, no error of clinical relevance), 6 - $10 \mathrm{mmHg}$ (slightly inaccurate), 11 - 15 $\mathrm{mmHg}$ (moderately inaccurate) and $>15 \mathrm{mmHg}$ (very inaccurate). The RRK measurements with the smallest differences were used for the comparison with the Nexfin ${ }^{\circledR}$ device.

In total the validation process (according to the original protocol(17)) consisted of two phases. The first phase determines how accurate the device will be for individual measurements and second phase 2.2 determines the accuracy for individual measurements. Determining the number of differences within 5, 10 and $15 \mathrm{mmHg}$, and then determining the accuracy will do this. (17)

To pass phase 1 there must be a minimum of respectively 65, 81 and 93 comparisons falling within 5, 10 and $15 \mathrm{mmHg}$. Furthermore, there must be a minimum of either 73 comparisons within $5 \mathrm{mmHg}$ and 87 comparisons within $10 \mathrm{mmHg}$, or 73 comparisons within $5 \mathrm{mmHg}$ and 96 comparisons within $15 \mathrm{mmHg}$, or 87 comparisons within $10 \mathrm{mmHg}$ and 95 comparisons within $96 \mathrm{mmHg}$. (17)

For phase 2 the number of comparisons falling within $5 \mathrm{mmHg}$ is calculated per subject. At least 24 of the 33 subjects must have at least two of their three comparisons lying within 5 mmHg. (17) Finally, visual inspection of Bland-Altman plots was performed to determine the agreement between the RRK BP measurements and the Nexfin ${ }^{\circledR}$ device. $(18,19)$

\section{POWER AND SAMPLE SIZE CALCULATION}

Both the original and the revised protocol of the European Society Of Hypertension recommend 33 test subjects for the validation of blood pressure monitoring devices. [15, 17] Therefore no (additional) power and sample size calculations were performed. 
TABLE 3.1. Overview of measurements by observers and Nexfin ${ }^{\circledR}$ device

\begin{tabular}{|l|l|c|c|}
\hline Measurement & & Systolic BP & Diastolic BP \\
\hline BPA & Entry RRK BP measurement & $\begin{array}{c}\text { A) } 136 \pm 13 \\
\text { B) } 141 \pm 18\end{array}$ & $\begin{array}{c}89 \pm 9 \\
91 \pm 11\end{array}$ \\
\hline BPB & Entry Nexfin ${ }^{\circledR}$ BP measurement & $130 \pm 21$ & $86 \pm 14$ \\
\hline BP1 & Two observers with RRK BP measurement & $\begin{array}{c}\text { A) } 136 \pm 16 \\
\text { B) } 140 \pm 17\end{array}$ & $91 \pm 10$ \\
\hline BP2 & With Nexfin ${ }^{\circledast}$ device & $131 \pm 25$ & $87 \pm 16$ \\
\hline BP3 & Two observers with RRK BP measurement & A) $133 \pm 14$ & $90 \pm 9$ \\
\hline BP4 & With Nexfin ${ }^{\circledast}$ device $139 \pm 18$ & $92 \pm 10$ \\
\hline BP5 & Two observers with RRK BP measurement & A) $133 \pm 15$ & $86 \pm 13$ \\
\hline BP6 & With Nexfin ${ }^{\circledast}$ device & B) $138 \pm 18$ & $91 \pm 10$ \\
\hline BP7 & Two observers with RRK BP measurement & A) $134 \pm 14$ & $88 \pm 12$ \\
\hline
\end{tabular}

Abbreviations: $B P=$ blood pressure $(m m H g), R R K=$ Riva-Rocci/Korotkoff, $A)=$ Observer $A, B)=$ Observer $B$

\subsection{RESULTS}

The present study was conducted between February and April 2016 according to the revised validation protocol of the European Society of Hypertension. [17] Figure 3.1 will elucidate the study process and table 3.1 gives an overview of the study measurements.

\section{PATIENT CHARACTERISTICS}

Table 3.2 gives an overview of the characteristics of the included patients. Of the 33 included patients, 3 had a history of cardiac disease (one myocardial infarction and two patients with stable angina), 4 had a history of thromboembolic disease (two patients with a pulmonary embolism and two with a deep venous thrombosis) 8 patients had a history of pulmonary disease (all of them had Chronic Obstructive Pulmonary Disease (COPD)) and 8 patients had a history of surgery (4 laparoscopic cholecystectomy, 2 laparoscopic appendectomy, 1 laparoscopic hysterectomy and 1 inguinal hernia repair). None of the included patients had hearth rhythm disorders, like atrial fibrillation.

\section{PHASE 1}

After completion of the measurements on all 33 patients all 99 comparisons between the RRK standard and the Nexfin ${ }^{\circledR}$ were analysed. Table 3.3 shows that the Nexfin ${ }^{\circledR}$ device did 
not pass the first phase of the revised validation protocol (17), with respectively 65,82 and 77 measurements in ranges within $5 \mathrm{mmHg}$, within $10 \mathrm{mmHg}$ and within $15 \mathrm{mmHg}$ for SBP and 61,76 and 90 measurements for DBP. Mean differences were $7.8 \pm 6.9 \mathrm{mmHg}$ for SBP and 8.0 $\pm 7.2 \mathrm{mmHg}$ for DBP, as shown in Bland Altman plots $(18,19)$ (Figure 3.1 and 3.2).

\section{PHASE 2}

In phase 2.2 the data was analysed per patient to determine the accuracy of the Nexfin ${ }^{\circledR}$ device for individual measurements. Of the 33 patients, 23 of them fell within the $5 \mathrm{mmHg}$ for SBP and 25 of them for DBP ( $\geq 22$ required to pass) respectively. Ten patients had all three of their comparisons over $5 \mathrm{mmHg}$ for SBP and 8 for DBP ( $\leq 3$ required to pass). (15) As shown in table 3.4, the Nexfin ${ }^{\circledast}$ device failed to pass phase 2. Figures 3.2 and 3.3 show Bland Altman plots for the analysis of the differences between respectively SBP and DBP measurements between the observers and Nexfin ${ }^{\circledR}$ values. $(18,19)$

TABLE 3.2. Patient characteristics

\section{Patients $(\mathrm{N}=33)$}

\begin{tabular}{|l|c|}
\hline Age (years) & $40 \pm 12$ \\
\hline Height $(\mathrm{cm})$ & $171 \pm 8.8$ \\
\hline Weight $(\mathrm{kg})$ & $125 \pm 21.9$ \\
\hline Waist circumference $(\mathrm{cm})$ & $42 \pm 6.4$ \\
\hline BMI $\left(\mathrm{kg} / \mathrm{m}^{2}\right)$ & \\
\hline & \\
\hline Comorbidities: & \\
\hline Diabetes Mellitus & 0 \\
\hline Hypertension & 0 \\
\hline Osteoarthritis & $13(39.4 \%)$ \\
\hline Obstructive Sleep Apnoea & $3(9.1 \%)$ \\
\hline Hypercholesterolemia & $5(15.2 \%)$ \\
\hline & \\
\hline Medical history: & \\
\hline Cardiac disease & $3(9.1 \%)$ \\
\hline Pulmonary disease & $8(24.2 \%)$ \\
\hline Thromboembolic & $4(12.1 \%)$ \\
\hline Previous surgery & $8(24.2 \%)$ \\
\hline & \\
\hline SBP (in mmHg) & $139 \pm 13$ \\
\hline DBP (in mmHg) & $90 \pm 7$ \\
\hline
\end{tabular}

Abbreviations: $\mathrm{BMI}=$ Body Mass Index, $\mathrm{SBP}=$ systolic blood pressure, $\mathrm{DBP}=$ diastolic blood pressure 
TABLE 3.3. Phase 1: - Performance requirements versus the actual performance of the Nexfin ${ }^{\circledR}$ according to the protocol of the European Society of Hypertension (17)

\begin{tabular}{|c|c|c|c|c|c|c|c|}
\hline \multicolumn{2}{|c|}{} & $\begin{array}{c}\text { Within } \\
\mathbf{5} \mathbf{~ m} \mathbf{m H g}\end{array}$ & $\begin{array}{c}\text { Within } \\
\mathbf{1 0} \mathbf{~ m} \mathbf{m H g}\end{array}$ & $\begin{array}{c}\text { Within } \\
\mathbf{1 5} \mathbf{~ m ~ m H g}\end{array}$ & Recommendation & $\begin{array}{c}\text { Mean } \\
\text { difference }\end{array}$ & $\begin{array}{c}\text { Standard } \\
\text { deviation }\end{array}$ \\
\hline & Two of & 73 & 87 & 96 & & & \\
& All of & 65 & 81 & 93 & & & \\
\hline Achieved & SBP & 65 & 82 & 77 & Fail & $7.8 \mathrm{mmHg}$ & $6.9 \mathrm{mmHg}$ \\
\hline & DBP & 61 & 76 & 90 & Fail & $8.0 \mathrm{mmHg}$ & $7.2 \mathrm{mmHg}$ \\
\hline
\end{tabular}

Abbreviations: $S B P=$ systolic blood pressure, $D B P=$ diastolic blood pressure

TABLE 3.4. Phase 2: - Performance requirements versus the actual performance of the Nexfin $₫$ according to the protocol of the European Society of Hypertension (17)

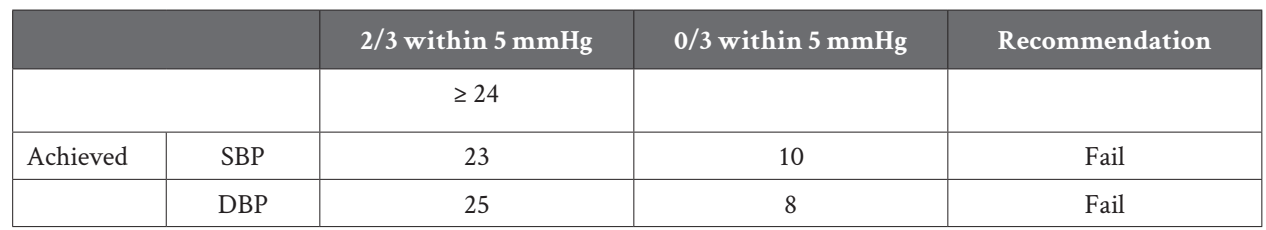

Abbreviations: $S B P=$ systolic blood pressure, $D B P=$ diastolic blood pressure 


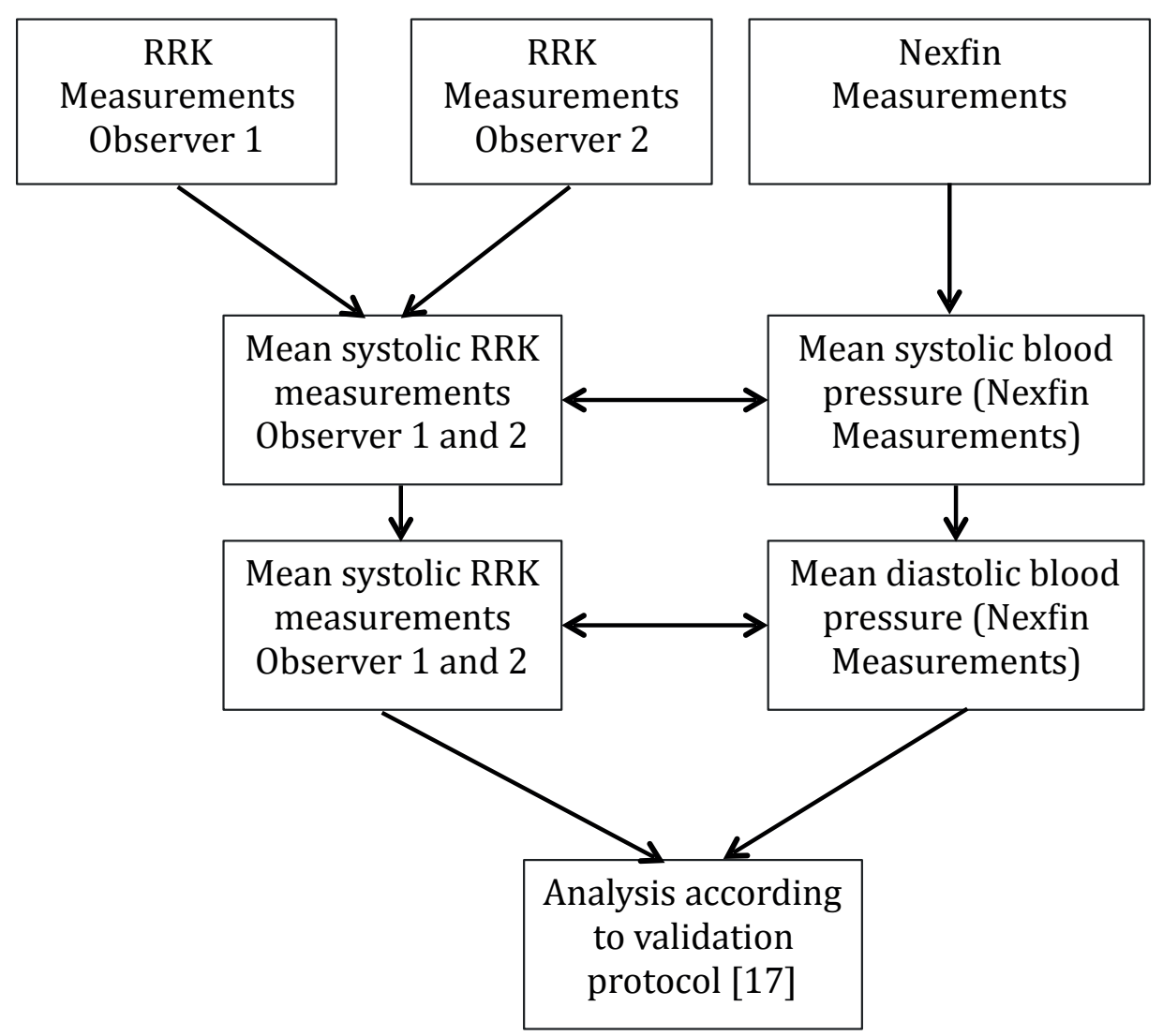

FIGURE 3.1. Graphic overview of the study process

$\leftrightarrow$ Comparison between the observed values between the Nexfin and the mean RRK blood pressure measurements between observer 1 and 2 


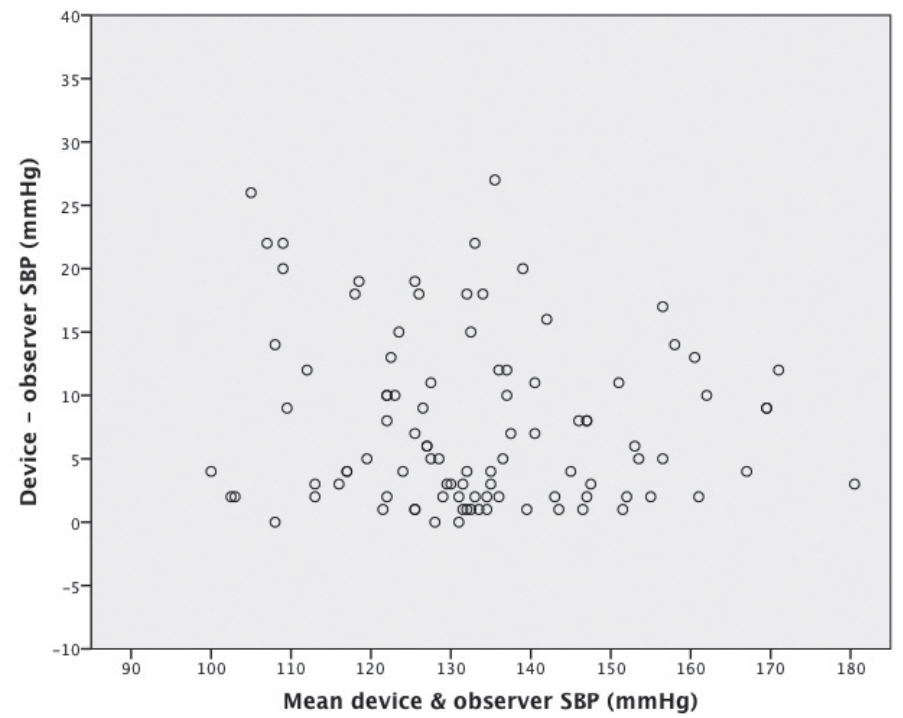

FIGURE 3.2: Bland-Altman plot showing the systolic blood pressure (SBP) differences between the Nexfin ${ }^{\circledR}$ device and the observer measurements (mean difference $7.8 \pm 6.9 \mathrm{mmHg}$, agreement interval between -6 and 21.6, 99 comparisons were used in the analysis)

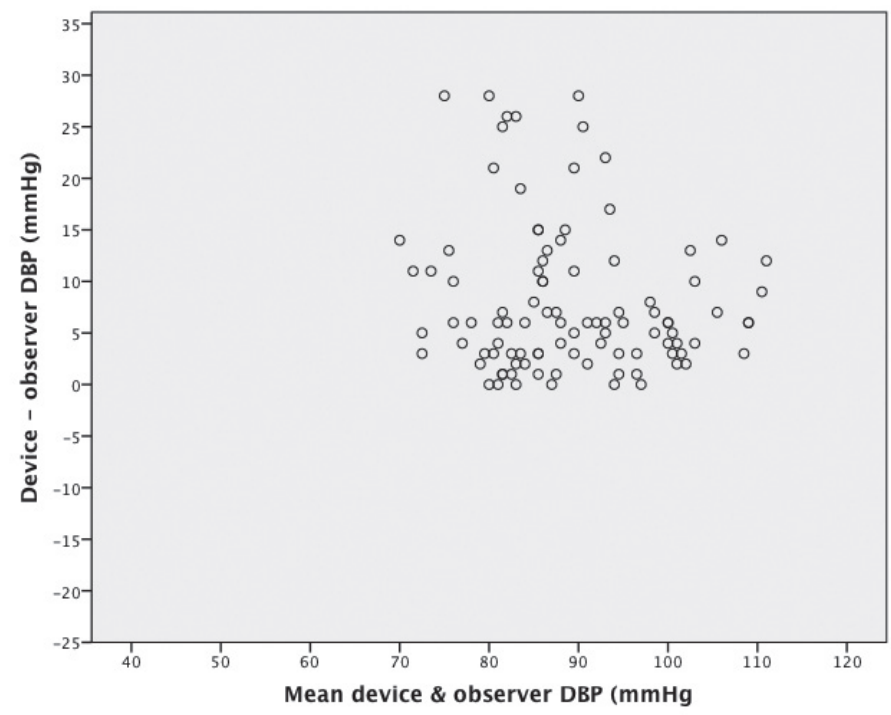

FIGURE 3.3: Bland-Altman plot showing the diastolic blood pressure (DBP) differences between the Nexfin ${ }^{\circledR}$ device and the observer measurements (mean difference $8.0 \pm 7.2 \mathrm{mmHg}$, agreement interval between -6.4 and 22.4, 99 comparisons were used in the analysis) 


\subsection{DISCUSSION}

In the present study we aimed to validate non-invasive continuous finger blood pressure measurements against classical sphyganomanometry blood pressure measurements in obese individuals. We have found that the Nexfin ${ }^{\circledR}$ device failed to pass the revised protocol for validation of the European Society Of Hypertension (17). When looking at the original validation protocol published in 2002 (15), several changes have been made. In the revised protocol published in 2010 phase 1 of the validation process was omitted and the criteria for phase 2.1 and 2.2 were sharpened.

Table 3.5 gives an overview of the criteria for validation according to the original protocol. (15) When comparing the obtained data with the revised protocol (17), the Nexfin ${ }^{\circledR}$ device was not able to pass part 1 and 2 of the validation (the former phase 2.1 and 2.2). These results are in line with a review by Stergiou and colleagues (20) assessing the effect of more stringent validation criteria on earlier validation studies. Not surprisingly, they found and increase in failure rate (from 17\% using the protocol of 2002 to $42 \%$ using the protocol published in 2010). However they state that a consistent trend toward improved performance of oscillometric devices assessed on the basis of the criteria established by the European Society of Hypertension and therefore by acknowledging the revised protocol this trend will allow more accurate devices to enter the market. $(20,21)$ These changes in validation criteria are the main reason why the Nexfin ${ }^{\circledR}$ device failed to pass the revised protocol for validation.

TABLE 3.5. Validation criteria according to the original protocol of the European Society of Hypertension published in 2002 (15)

\begin{tabular}{|c|c|c|c|c|c|}
\hline & & $\begin{array}{l}\text { Within } \\
5 \mathrm{mmHg}\end{array}$ & $\begin{array}{l}\text { Within } \\
10 \mathrm{mmHg}\end{array}$ & $\begin{array}{l}\text { Within } \\
15 \mathrm{mmHg}\end{array}$ & Recommendation \\
\hline Phase 1 & One of & 25 & 35 & 40 & \\
\hline \multirow[t]{2}{*}{ Phase 2.1} & Two of & 65 & 80 & 95 & \\
\hline & All Of & 60 & 75 & 90 & \\
\hline \multirow[t]{2}{*}{ Phase $2.2 *$} & & $2 / 3$ within $5 \mathrm{mmHg}$ & $0 / 3$ with $5 \mathrm{mmHg}$ & & \\
\hline & & $\geq 22$ & $\leq 3$ & & \\
\hline
\end{tabular}

Abbreviations: $\mathrm{SBP}=$ systolic blood pressure, $\mathrm{DBP}=$ diastolic blood pressure

* Individual measurements

However, we think that the use of $\mathrm{Nexfin}^{\circledR}$ device can be valuable in a population of obese patients scheduled for bariatric surgery. Both protocols $(15,17)$ do not make any recommendations for special groups like patients with obesity. However, by selection of obese patients with a BMI $42.8 \pm 6.4 \mathrm{~kg} / \mathrm{m}^{2}(10)$ and by exclusion of hypertension and type 
2 diabetes mellitus, our group should be sufficiently uniform to enable application of the protocol. $(15,17)$

We focused on obese patients scheduled for bariatric surgery, because hypertension is one of the comorbidities associated with (morbid) obesity (22) and evaluating the effects of bariatric surgery on hypertension is clinically important for bariatric and metabolic surgery. $(22,23)$ It has been recognized that RRK BP measurements can give an overestimation of the BP of obese patients due to the cuff size and the circumference of the upper arm. $(24,25)$ This can lead to misdiagnosing and potential over-treating hypertension in this population. (24-26) Although it is recommended to use a larger cuffs in obese individuals to ensure a reliable ratio between the cuff width and the upper arm circumference for reliable blood pressure measurements. (26) However, these recommendations in clinical practice are not always applied and overestimation of the BP can occur. Secondly we hypothesize that the blood pressure at the finger level gives a better representation of the brachial blood pressures (RRK measurements) in obese patients. Therefore non-invasive beat-to-beat BP monitoring could be a valuable addition to standard care for obese patients.

The overestimation of RRK BP measurements in obese patients is supported by several studies. (24-26) Conceivably, the systolic BP recorded at the wrist may better reflect 'true' systolic BP prevailing in the artery than the systolic BP measured at the arm. (27) This possibility is supported in the study by de Senarclens et al. (27) by the observation that the ratio of width (the decimal log arm of circumference calculated in obese patients) was higher than the optimal value originally validated by Marks et al. (26) This basically means that in our study the arm systolic blood pressure is an overestimation of the 'true' blood pressure. Notwithstanding these results, small BP differences may exist between the brachial artery and the radial artery, ranges of 1-4 mmHg. (28) So basically in our study population the blood pressure measured with the Nexfin ${ }^{\circledR}$ device is he 'true blood pressure.' Secondly, the overestimation of the BP of our study population (because we used RRK BP measurements as reference) can also be a reason that the $\mathrm{Nexfin}^{\circledR}$ device did not pass the validation protocol. This also explains why there is less differences between the Nexfin ${ }^{\circledR}$ measurements and other reference methods (such as radial artery blood pressure, used in a recent study by De Wilde et al. (9)) Thirdly, we have to take into account that the shape of a blood pressure wave constantly distorts as it travels from the central elastic arteries toward the muscular conduit arteries. This physiological phenomenon is known as pulse wave amplification, that the blood pressure, as a periodically oscillating wave, travels and reflects in occasionally differently structured portions of the viscoelastic arterial system. In healthy individuals, the pulse wave amplitude (pulse pressure (PP)) increases from the aorta/carotid section to the brachial/radial section without added energy, such that the arterial central pressure and the diastolic pressure remains almost unchanged. 
Nowadays several strategies for BP measurement exist, of which intra-arterial BP measurement (mostly in the radial artery) is considered to be the most accurate. (16) Because of its invasiveness and its technical difficulty in the obese population, risk for infection it is not suitable for routine BP measurement in obese patients scheduled for bariatric surgery. Secondly, we have the standard auscultatory sphygmomanometry, widely used in clinical practice, based on the principles of Riva-Rocci/Korotkoff. (16) Thirdly, there is a wide range of automated devices (the oscillometric type) that provide BP measurements of one systolic and diastolic beat. $(15,16)$ Finally, finger blood pressure measurements devices, such as $\mathrm{Nexfin}^{\circledR}$, derive non-invasive continuous blood pressure waveforms from pressure waveforms in the finger using algorithms to calculates the beat-to-beat brachial BP. $(2-4,8)$

\section{LIMITATIONS:}

Firstly, the simultaneous measurement of the Nexfin ${ }^{\circledR}$ device and the reference method on the ipsilateral arm is, for obvious reasons, not possible, and the spontaneous variability in BP will thus contribute to the error. Nonetheless, ipsilateral measurements are preferred to contralateral measurements since an unknown left to-right difference might also afflict the comparison. Another disadvantage is that the RRK reference measurement itself often disturbs the test measurement: with temporal occlusion of the brachial artery, the BP distal to the arm cuff becomes strongly reduced. Subsequent release of the arm cuff may affect finger arterial pressure measurements by inducing a post occlusion hyperemic response. $(6-8,12)$

\subsection{CONCLUSION}

This study aimed to validate the Nexfin ${ }^{\circledast}$ monitor against classical sphyganomanometry (Riva-Rocci/Korotkoff (RRK)) blood pressure measurements in patients with obesity scheduled for bariatric surgery, using the validation protocol developed by the European Society of Hypertension. Using the revised protocol (17) the Nexfin ${ }^{\circledR}$ device was not able to pass validation. Using the original protocol (15), the Nexfin ${ }^{\circledR}$ device passed phase 1 and 2.1 of the validation process, but failed to pass phase 2.2. However the Nexfin ${ }^{\otimes}$ device could be of value in clinical settings and/or research settings as blood pressure measurement device. 


\subsection{REFERENCES}

1. Rongen GA, Bos WJ, Lenders JW, van Montfrans GA, van Lier HJ, van Goudoever J, et al. Comparison of intrabrachial and finger blood pressure in healthy elderly volunteers. American journal of hypertension. 1995;8(3):237-48.

2. Wesseling KH, De Wit B, Van der Hoeven GMA, Van Goudoever J, Settels JJ. Physiocal, calibrating finger vascular physiology for Finapres. Homeostasis. 1995;36:67-82.

3. Penaz J, editor Photoelectric measurement of blood pressure, volume and flow in the fingerDigest 10th International Conference of Medical Biological Engineering; . Dresden1973.

4. Wesseling KH. A century of noninvasive arterial pressure measurement: from Marey to Penaz and Finapres. Homeostasis. 1995;36:2-3.

5. Immink RV, van den Born BJ, van Montfrans GA, Koopmans RP, Karemaker JM, van Lieshout JJ. Impaired cerebral autoregulation in patients with malignant hypertension. Circulation. 2004;110(15):2241-5.

6. Imholz BP, Settels JJ, van der Meiracker AH, Wesseling KH, Wieling W. Non-invasive continuous finger blood pressure measurement during orthostatic stress compared to intra-arterial pressure. Cardiovascular research. 1990;24(3):214-21.

7. Imholz BP, Wieling W, van Montfrans GA, Wesseling KH. Fifteen years experience with finger arterial pressure monitoring: assessment of the technology. Cardiovascular research. 1998;38(3):605-16.

8. Eeftinck Schattenkerk DW, van Lieshout JJ, van den Meiracker AH, Wesseling KR, Blanc S, Wieling W, et al. Nexfin noninvasive continuous blood pressure validated against Riva-Rocci/ Korotkoff. American journal of hypertension. 2009;22(4):378-83.

9. de Wilde RB, de Wit F, Geerts BF, van Vliet AL, Aarts LP, Vuyk J, et al. Non-invasive continuous arterial pressure and pulse pressure variation measured with $\operatorname{Nexfin}((\mathrm{R}))$ in patients following major upper abdominal surgery: a comparative study. Anaesthesia. 2016;71(7):788-97.

10. Ng M, Fleming T, Robinson M, Thomson B, Graetz N, Margono C, et al. Global, regional, and national prevalence of overweight and obesity in children and adults during 1980-2013: a systematic analysis for the Global Burden of Disease Study 2013. Lancet (London, England). 2014;384(9945):766-81.

11. Westerhof BE, Guelen I, Parati G, Groppelli A, van Montfrans GA, Wieling W, et al. Variable day/ night bias in 24-h non-invasive finger pressure against intrabrachial artery pressure is removed by waveform filtering and level correction. Journal of hypertension. 2002;20(10):1981-6.

12. Gizdulich P, Imholz BP, van den Meiracker AH, Parati G, Wesseling KH. Finapres tracking of systolic pressure and baroreflex sensitivity improved by waveform filtering. Journal of hypertension. 1996;14(2):243-50.

13. Gizdulich P, Prentza A, Wesseling KH. Models of brachial to finger pulse wave distortion and pressure decrement. Cardiovascular research. 1997;33(3):698-705.

14. Bogert LW, Harms MP, Pott F, Secher NH, Wesseling KH, van Lieshout JJ. Reconstruction of brachial pressure from finger arterial pressure during orthostasis. Journal of hypertension. 2004;22(10):1873-80. 
15. O’Brien E, Pickering T, Asmar R, Myers M, Parati G, Staessen J, et al. Working Group on Blood Pressure Monitoring of the European Society of Hypertension International Protocol for validation of blood pressure measuring devices in adults. Blood pressure monitoring. 2002;7(1):317.

16. Pickering TG, Hall JE, Appel LJ, Falkner BE, Graves J, Hill MN, et al. Recommendations for blood pressure measurement in humans and experimental animals: Part 1: blood pressure measurement in humans: a statement for professionals from the Subcommittee of Professional and Public Education of the American Heart Association Council on High Blood Pressure Research. Hypertension. 2005;45(1):142-61.

17. O’Brien E, Atkins N, Stergiou G, Karpettas N, Parati G, Asmar R, et al. European Society of Hypertension International Protocol revision 2010 for the validation of blood pressure measuring devices in adults. Blood pressure monitoring. 2010;15(1):23-38.

18. Giavarina D. Understanding Bland Altman analysis. Biochemia medica. 2015;25(2):141-51.

19. Bland JM, Altman DG. Statistical methods for assessing agreement between two methods of clinical measurement. Lancet (London, England). 1986;1(8476):307-10.

20. Stergiou GS, Karpettas N, Atkins N, O’Brien E. Impact of applying the more stringent validation criteria of the revised European Society of Hypertension International Protocol 2010 on earlier validation studies. Blood pressure monitoring. 2011;16(2):67-73.

21. Stergiou GS, Karpettas N, Atkins N, O’Brien E. European Society of Hypertension International Protocol for the validation of blood pressure monitors: a critical review of its application and rationale for revision. Blood pressure monitoring. 2010;15(1):39-48.

22. Buchwald H, Avidor Y, Braunwald E, Jensen MD, Pories W, Fahrbach K, et al. Bariatric surgery: a systematic review and meta-analysis. Jama. 2004;292(14):1724-37.

23. Careaga M, Esmatjes E, Nunez I, Molero J, Vidal J, Flores L. Effect of weight loss on abnormal 24hour blood pressure patterns in severely obese patients. Surgery for obesity and related diseases : official journal of the American Society for Bariatric Surgery. 2015.

24. Maxwell MH, Waks AU, Schroth PC, Karam M, Dornfeld LP. Error in blood-pressure measurement due to incorrect cuff size in obese patients. Lancet (London, England). 1982;2(8288):33-6.

25. Linfors EW, Feussner JR, Blessing CL, Starmer CF, Neelon FA, McKee PA. Spurious hypertension in the obese patient. Effect of sphygmomanometer cuff size on prevalence of hypertension. Archives of internal medicine. 1984;144(7):1482-5.

26. Marks LA, Groch A. Optimizing cuff width for noninvasive measurement of blood pressure. Blood pressure monitoring. 2000;5(3):153-8.

27. de Senarclens O, Feihl F, Giusti V, Engelberger RP, Rodieux F, Gomez P, et al. Brachial or wrist blood pressure in obese patients: which is the best? Blood pressure monitoring. 2008;13(3):14951.

28. O’Rourke MF, Kelly R, Avolio A. The arterial pulse. . Philadelphia-London: Lea \& Febiger; 1992. 



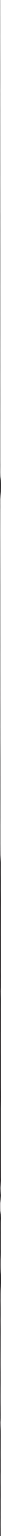




\title{
4. SHORT-TERM CHANGES IN CARDIOVASCULAR
} HEMODYNAMICS IN RESPONSE TO BARIATRIC SURGERY AND WEIGHT LOSS USING THE NEXFIN ${ }^{\circledR}$ NON-INVASIVE CONTINUOUS MONITORING DEVICE: A PILOT STUDY

\author{
Sjaak Pouwels ${ }^{1,2}$ \\ Bianca Lascaris ${ }^{3}$ \\ Simon W. Nienhuijs ${ }^{1}$ \\ R. Arthur Bouwman ${ }^{3}$ \\ Marc P. Buise ${ }^{3}$
}

Accepted for publication in Obesity Surgery

1. Department of Surgery, Catharina Hospital, Eindhoven, The Netherlands

2. Department of Epidemiology, CAPHRI Research School, Maastricht University, The Netherlands

3. Department of Anaesthesiology, Intensive Care and Pain Medicine, Catharina Hospital, Eindhoven, The Netherlands 


\section{ABSTRACT}

\section{Background}

Compared to healthy individuals, obese have significantly higher systolic and diastolic blood pressure; mean arterial pressure, heart rate and cardiac output. Aim of this study was to evaluate cardiovascular hemodynamic changes before and 3 months after bariatric surgery.

\section{Methods}

Patients scheduled for bariatric surgery between the 29th of September 2016 and $24^{\text {th }}$ of March 2016 were included and compared with 24 healthy individuals. Hemodynamic measurements were performed preoperatively and 3 months after surgery, using the Nexfin ${ }^{\circledast}$ non-invasive continuous hemodynamic monitoring device (Edwards Lifesciences / BMEYE B.V., Amsterdam, the Netherlands).

\section{Results}

Eighty subjects were included in this study, respectively 56 obese patients scheduled for bariatric surgery and 24 healthy individuals. Baseline hemodynamic measurements showed significant differences in cardiac output ( $6.5 \pm 1.6$ versus $5.7 \pm 1.6 \mathrm{l} / \mathrm{min}, \mathrm{p}=0.046)$, mean arterial pressure ( $107 \pm 19$ versus $89 \pm 11 \mathrm{mmHg}, \mathrm{p}=0.001)$, systolic (134 \pm 24 versus $116 \pm 18$ $\mathrm{mmHg}, \mathrm{p}=0.001)$ and diastolic blood pressure $(89 \pm 17$ versus $74 \pm 10 \mathrm{mmHg}, \mathrm{p}=0.001)$ and heart rate $(87 \pm 12$ versus $76 \pm 14 \mathrm{bpm}, \mathrm{p}=0.02)$ between obese and healthy subjects. Three months after surgery, significant changes occurred in mean arterial pressure $(89 \pm 17 \mathrm{mmHg}$, $\mathrm{p}=0.001)$, systolic $(117 \pm 24 \mathrm{mmHg}, \mathrm{p}=0.001)$ and diastolic blood pressure $(71 \pm 15 \mathrm{mmHg}$, $\mathrm{p}=0.001)$, stroke volume $(82.2 \pm 22.4 \mathrm{ml}, \mathrm{p}=0.03)$ and heart rate $(79 \pm 17 \mathrm{bpm}, \mathrm{p}=0.02)$.

\section{Conclusion}

Three months after bariatric surgery significant improvements occur in hemodynamic variables except cardiac output and cardiac index, in the patient group. 


\subsection{INTRODUCTION}

Obesity is a recognized risk factor for cardiac dysfunction, atherosclerosis and cardiovascular disease. $(1,2)$ When combined with hypertension, dyslipidaemia and type 2 diabetes mellitus, it is known as 'metabolic syndrome', which in itself is an independent predictor of cardiac dysfunction and cardiovascular disease. (1, 3) Increasing evidence from large population based studies (such as the Framingham Heart Study) and cardiac imaging trials show that long-term obesity can lead to structural changes in cardiac structure, such as left ventricular (LV) dilatation, hypertrophy and even heart failure. $(1,4)$ Adipose tissue itself contributes to the circulating volume and must increase cardiac output. (5)

The literature suggests that weight loss is able to improve cardiac structure and function, decrease cardiovascular risk (due to reverse remodelling). (6,7) Aggarwal et al. (6) summarised the beneficial effects of bariatric procedures on cardiac functional imaging endpoints, but short-term hemodynamic changes have not been studied that extensively. Namely the presence of left ventricular hypertrophy $(\mathrm{LVH})$ and hypertension are a prerequisite for cardiac remodelling after bariatric surgery, and in this review by Aggarwal et al. (6) mostly cardiac structure changes were evaluated, without looking at the short-term hemodynamic changes after bariatric surgery.

The aim of the present study was to evaluate the cardiovascular hemodynamic changes before and 3 months after bariatric surgery. It was hypothesized that bariatric surgery (irrespective of the type of surgical procedure) gives a decrease in cardiac output and in systolic and diastolic blood pressure.

\subsection{METHODS}

This study was approved by the Medical Ethics Committee of the Catharina Hospital in Eindhoven, The Netherlands and adheres the principles of the declaration of Helsinki. Informed consent was obtained of all patients included. This study was conducted according to the Strengthening the Reporting of Observational studies in Epidemiology (STROBE) statement. (8)

\section{PATIENT POPULATION}

A multidisciplinary team screened all included patients preoperatively, consisting of a physician assistant, a psychologist, a nutritionist and a surgeon. Patients were eligible for surgery if they had a BMI of $40 \mathrm{~kg} / \mathrm{m}^{2}$ or higher, or a BMI between 35 and $40 \mathrm{~kg} / \mathrm{m}^{2}$ with 
significant co-morbidities, with serious attempts to lose weight in the past. Co-morbidities were considered significant when medication had to be used, or if continuous positive airway pressure (CPAP) had to be used in case of obstructive sleep apnoea (OSAS). Patients with pre-existing cardiac and/or pulmonary and/or kidney diseases were excluded from this study.

The subjects in this study were divided into two groups. Group A of morbidly obese patients scheduled for bariatric surgery that were measured preoperatively and three months after bariatric surgery. Group B was a control group of, healthy, health professionals recruited from the research team..

\section{NEXFIN ${ }^{\circledR}$ HEMODYNAMIC MONITORING DEVICE}

The Nexfin ${ }^{\circledast}$ device (Edwards Lifesciences/BMEYE B.V., Amsterdam, the Netherlands) is based on the volume clamp method first described by Penaz and the Physiocal criteria of Wesseling, and provides a reconstructed continuous brachial arterial BP that are used to derive stroke volume. (9-14)

\section{PROCEDURE AND DATA COLLECTION}

All hemodynamic measurements were obtained before and only the patient's measurements were repeated 3 months after bariatric surgery. Patients were asked to relax for 10 minutes in a comfortable chair and any form of disturbance was prevented. After that each study participant was asked neither to speak nor to move during the measurement. Appropriate cuff size was determined for both the finger and the arm in each participant. The Nexfin ${ }^{\circledast}$ finger cuff was attached to the mid-phalanx of the third digit of the patients left hand. Data of interest were anthropometric variables and comorbidities, hemodynamic variables (systolic and diastolic blood pressure, cardiac output, cardiac index, stroke volume and heart rate). Secondly, operative details and evolution of weight and comorbidities were recorded, in terms of remission of type 2 diabetes, hypertension, hypercholesterolemia, sleep apnoea and osteoarthritis.

Primary endpoint of the study was the change in cardiac output, cardiac index and systolic and diastolic pressure 3 months after bariatric surgery. Secondary endpoints were other hemodynamic parameters such as stroke volume and heart rate.

\section{STATISTICAL ANALYSIS}

Our hypothesis was that bariatric surgery gives a decrease in cardiac output and in systolic and diastolic blood pressure. In current literature, it is seen that bariatric surgery gives a mean reduction of $20 \%$ in systolic and diastolic blood pressure. $(3,15)$ 
Using a two-sided 5\% significance level and a power of $90 \%$, a power analysis indicated a sample of 50 patients. Taking into account a dropout rate of $10 \%$ we have included 56 patients.

Continuous variables were shown as mean \pm standard deviation (SD) and categorical variables as frequency with percentages. The Shapiro-Wilk test was used to test each variable for normality. Student's t-test for independent groups or the Mann-Whitney U test was used to compare the hemodynamic variables in the preoperative period, depending on the normality or non-normality of the data distribution. To determine whether the hemodynamic variables 3 months reach normal values, these follow-up values were compared with the baseline values of group $B$ (the health professionals), assuming these measurements would not change in 3 months. In all tests, values of $p<0.05$ were considered statistically significant. Statistical Package for Social Sciences (SPSS, Chicago, IL, USA Version 20.0) was used to prepare the database and for statistical analysis.

\subsection{RESULTS}

A total of 80 subjects were included in this study, respectively 54 patients in group A, and 24 healthy individuals in group B. Three months after surgery, follow-up data was available of 51 patients in group A. Three patients were unable to attend the follow-up visits at our clinic; one was admitted in a hospital because of a cerebral infarction and two patients were lost to follow-up. Table 4.1 gives an overview of the characteristics of each study group. A significant difference was found between group A and group B in terms of weight, waist circumference and body mass index (BMI).

\section{WEIGHT LOSS AND RESOLUTION OF COMORBIDITIES}

Table 4.1 gives an overview of the weight loss profiles in group A after three months, the group of patients that underwent bariatric surgery. In total 29 patients (53.7\%) had a Rouxen-Y gastric bypass (RYGB), 25 (46.3\%) underwent a Sleeve Gastrectomy (SG). In terms of resolution of comorbidities after three months; 15 patients (12.7\%) had partial remission and 3 patients $(2.5 \%)$ had complete remission of hypertension. Hypercholesterolemia resolved partially in 5 patients $(4.2 \%)$ and completely in 6 patients (5.1\%). Obstructive sleep apnoea syndrome (OSAS) resolved partially in 7 patients (5.9\%) and completely in 2 patients $(1.7 \%)$. The remission of Type 2 diabetes (T2DM) was partial in 8 patients $(6.8 \%)$ and complete in 3 patients (2.5\%). 


\section{BASELINE CARDIOVASCULAR HEMODYNAMIC VARIABLES}

Table 4.2 gives an overview of the baseline hemodynamic variables between groups $\mathrm{A}$ and B. Between groups A and B no significant differences were found in stroke volume (SV) and cardiac index (CI)

TABLE 4.1. Demographics of the study population

\begin{tabular}{|c|c|c|c|}
\hline & Group A (N=54) & Group B (N=24) & P-Value \\
\hline Age (years) & $44 \pm 11$ & $37 \pm 13$ & 0.09 \\
\hline Gender (Male: Female) & $12 / 42$ & $10 / 14$ & 0.107 \\
\hline Height $(\mathrm{cm})$ & $168.9 \pm 9.3$ & $175.5 \pm 8.2$ & 0.08 \\
\hline Weight (kg) & $119.9 \pm 21.6$ & $77.2 \pm 16.6$ & 0.01 \\
\hline Waist circumference $(\mathrm{cm})$ & $128.4 \pm 15.4$ & $85.6 \pm 15.1$ & 0.01 \\
\hline BMI $\left(\mathrm{kg} / \mathrm{m}^{2}\right)$ & $41.9 \pm 5.7$ & $25.1 \pm 5.2$ & 0.01 \\
\hline \multicolumn{4}{|l|}{ Comorbidities: } \\
\hline Diabetes Mellitus & $11(19.6 \%)$ & $1(4.2 \%)$ & 0.643 \\
\hline Hypertension & $22(39.3 \%)$ & $1(4.2 \%)$ & 0.386 \\
\hline Osteoarthritis & $44(78.6 \%)$ & $1(4.2 \%)$ & 0.367 \\
\hline Obstructive Sleep Apnoea & $10(17.9 \%)$ & $1(4.2 \%)$ & 0.565 \\
\hline Hypercholesterolemia & $12(21.4 \%)$ & $1(4.2 \%)$ & 0.181 \\
\hline \multicolumn{4}{|l|}{ Medical history: } \\
\hline Cardiac disease & $2(3.6 \%)$ & & 0.645 \\
\hline Pulmonary disease & $5(8.9 \%)$ & & 0.573 \\
\hline Thromboembolic & $7(12.5 \%)$ & & 0.486 \\
\hline \multicolumn{4}{|l|}{ Type of bariatric surgery } \\
\hline RYGB & $29(51.8 \%)$ & & \\
\hline SG & $25(44.6 \%)$ & & \\
\hline \multicolumn{4}{|l|}{ Follow-up } \\
\hline Weight (kg) & $97.4 \pm 18.9$ & & \\
\hline Waist circumference $(\mathrm{cm})$ & $102.8 \pm 14.7$ & & \\
\hline $\operatorname{BMI}\left(\mathrm{kg} / \mathrm{m}^{2}\right)$ & $34.0 \pm 5.2$ & & \\
\hline Absolute weight loss $(\mathrm{kg})$ & $-22.5 \pm 5.7$ & & \\
\hline$\% \mathrm{TWL}$ & $-18.8 \% \pm 4.1$ & & \\
\hline$\%$ EWL & $-41.5 \% \pm 58$ & & \\
\hline \%BMIL $\left(\mathrm{kg} / \mathrm{m}^{2}\right)$ & $-22.5 \% \pm 5.7$ & & \\
\hline
\end{tabular}

Abbreviations: BMI = Body Mass Index, RYGB = Roux-en-Y Gastric Bypass, SG = Sleeve Gastrectomy, \%TWL $=\%$ Total Weight Loss, \%EWL $=\%$ Excess Weight Loss, \%BMIL $=\%$ BMI Loss 
TABLE 4.2. Baseline hemodynamic variables of each study group

\begin{tabular}{|c|c|c|c|}
\hline & Group A (N=54) & Group B $(\mathrm{N}=24)$ & P-Value \\
\hline SBP $(\mathrm{mmHg})$ & $134 \pm 24$ & $116 \pm 18$ & 0.001 \\
\hline DBP (mmHg) & $89 \pm 17$ & $74 \pm 10$ & 0.001 \\
\hline MAP (mmHg) & $107 \pm 19$ & $89 \pm 11$ & 0.001 \\
\hline $\mathrm{CO}(\mathrm{L} / \mathrm{min})$ & $6.5 \pm 1.6$ & $5.7 \pm 1.6$ & 0.046 \\
\hline $\mathrm{CI}\left(\mathrm{L} / \mathrm{min} / \mathrm{m}^{2}\right)$ & $2.9 \pm 0.8$ & $3.0 \pm 0.9$ & 0.937 \\
\hline $\mathrm{SV}(\mathrm{ml})$ & $75.5 \pm 16.7$ & $75.6 \pm 16.7$ & 0.930 \\
\hline HR (bpm) & $87 \pm 12$ & $76 \pm 14$ & 0.002 \\
\hline
\end{tabular}

Abbreviations: $S B P=$ Systolic Blood Pressure, $D B P=$ Diastolic Blood Pressure, $M A P=$ Mean Arterial Pressure, $C O=$ Cardiac Output, $C I=$ Cardiac Index, $S V=$ Stroke Volume, $H R=$ Heart Rate

TABLE 4.3. Changes in cardiovascular hemodynamics measured with the Nexfin ${ }^{\circledR}$ device, 3 months after bariatric surgery

\begin{tabular}{|l|c|c|c|}
\hline Group A & Preoperative (N=54) & Postoperative (N=51) & P-Value \\
\hline SBP $(\mathrm{mmHg})$ & $134 \pm 24$ & $117 \pm 24$ & $<\mathbf{0 . 0 0 1}$ \\
\hline DBP $(\mathrm{mmHg})$ & $89 \pm 17$ & $71 \pm 15$ & $<\mathbf{0 . 0 0 1}$ \\
\hline MAP $(\mathrm{mmHg})$ & $107 \pm 19$ & $89 \pm 17$ & $<\mathbf{0 . 0 0 1}$ \\
\hline CO $(\mathrm{L} / \mathrm{min})$ & $6.5 \pm 1.6$ & $5.9 \pm 2.1$ & 0.082 \\
\hline CI $\left(\mathrm{L} / \mathrm{min} / \mathrm{m}^{2}\right)$ & $2.9 \pm 0.8$ & $2.9 \pm 1.1$ & 0.970 \\
\hline SV $(\mathrm{ml})$ & $75.5 \pm 16.7$ & $82.2 \pm 22.4$ & $\mathbf{0 . 0 3}$ \\
\hline HR $(\mathrm{bpm})$ & $87 \pm 12$ & $79 \pm 17$ & $<\mathbf{0 . 0 0 1}$ \\
\hline
\end{tabular}

Abbreviations: $S B P=$ Systolic Blood Pressure, $D B P=$ Diastolic Blood Pressure, $M A P=$ Mean Arterial Pressure, $C O=$ Cardiac Output, $C I=$ Cardiac Index, $S V=$ Stroke Volume, $H R=$ Heart Rate

\section{EFFECT OF WEIGHT LOSS ON CARDIOVASCULAR HEMODYNAMIC VARIABLES}

Follow-up data was available of 51 patients (in group A). Table 4.3 gives an overview the changes in cardiovascular hemodynamic variables. There were no differences between postoperative and preoperative cardiac output $(\mathrm{CO})$ and cardiac index $(\mathrm{CI})$ (p-values respectively 0.082 and 0.970 ). Compared to group B, the postoperative hemodynamics of group A did not differ significantly from that of the healthy individuals. (Respectively $\mathrm{p}=0.586$ (Systolic Blood Pressure); 0.678 (Diastolic Blood Pressure); 0.778 (Mean Arterial Pressure); 0.335 (Cardiac Output); 0.246 (Cardiac Index); 0.890 (Stroke Volume) and 0.145 (Heart Rate).

We did a subgroup analysis to determine if there is a relationship between the type of bariatric surgery and the postoperative changes in cardiovascular hemodynamics. No significant differences were found between patients who underwent an SG or a RYGB of revisional surgery. 


\subsection{DISCUSSION}

The present study assessed the effects of bariatric surgery on cardiovascular hemodynamics 3 months after bariatric surgery We have found that compared to healthy individuals, obese patients prior to bariatric surgery have significantly higher systolic and diastolic blood pressure, mean arterial pressure, heart rate and cardiac output. Three months after bariatric surgery significant improvements occur in all hemodynamic variables except cardiac output and cardiac index. Secondly, assuming that hemodynamic measurements of healthy individuals do not change over the course of three months, we do not see a significant difference in postoperative hemodynamics compared to healthy individuals that might be a clue that there is a normalisation of hemodynamic profile after 3 months.

With respect to hemodynamic changes after bariatric surgery, conflicting results are reported in current literature. Several studies report a significant decrease in systolic and diastolic blood pressure in the first weeks after bariatric surgery, but also over longer postoperative period. (16-23) A few reports only show a significant decrease in either mean arterial blood pressure $(24,25)$, a long-term significant decrease in systolic blood pressure (26-29) or a significant decrease in diastolic blood pressure. (30-32) In contrast to these positive results, others show no significant changes in blood pressure after bariatric surgery. (25, 27, 32-41) This may be explained by presence of hypertension and/or a high left ventricular mass (LVM) as postulated by Mukerji et al. (42) and Alpert et al. (43). Furthermore, a few studies found a significant short- and long-term decrease in heart rate after bariatric surgery, $(16,21,23,25$, $28-32,35,36,44)$ while others found no differences.. (24, 33, 34, 37-39, 41, 42, 45) The most intriguing fact of these results is that there must be an intrinsic morphological factor than makes the difference and explains us why these conflicting results exist. In current literature cardiac remodelling after bariatric surgery is seen mostly in patients with hypertension, LVH or both. (43) In our opinion, pre-existing hypertension or left ventricular hypertrophy might be prerequisite for reverse remodelling after bariatric surgery and thus the explanation for the conflicting results in current literature.

Regarding cardiac output we hypothesized that, due to volume decrease as result of weight loss, the cardiac output would decrease three months after surgery. Our data suggest that a slight decrease in cardiac output occurs, although significance was not reached ( $p=0.082$ ). Moreover, in our patient population the heart rate decreased and stroke volume increased 3 month after bariatric surgery significantly. However there was a decreasing trend in cardiac output seen in our study, although not significant. This observation is in line with current literature, because in most of the studies a significant decrease after 6-24 months and even after 10 years in terms of cardiac output was seen. $(21,25,28,32,41,44)$ These changes might 
indicate an improvement in relative volume overload, which can be the start of normalization of cardiac remodelling. However the degree of remodelling in relationship with the time postoperative is not known.

The findings of cardiovascular changes after bariatric surgery are noticeable (6) and may represent a supra-physiological effect of metabolic/bariatric surgical procedures. (1) There might be a combination of weight-dependent (e.g. weight loss) and also weight-independent mechanisms (inotropic hormones, like GLP-1) that induce cardiac remodelling. This includes that there is a possibility of direct gut hormonal inotropic action on the myocardium through an enterocardiac axis. $(1,6)$ The classical hemodynamic weight-dependent effect of bariatric surgery is no longer thought to be the only one responsible for reverse remodelling seen after bariatric surgery, as the beneficial effects can be independent from changes in blood pressure. $(15,30)$

Manipulation of enteric gut hormones has been shown to have beneficial effects on cardiovascular function through the enterocardiac axis. $(1,46)$ Hormones such as secretin, glucagon and vasoactive intestinal peptide, act as inotropes by activating cardiac membrane adenylate cyclase, which is a key enzyme in cardiac cellular energy physiology. (47) Although these mechanisms are not fully understood, it is thought that the energy metabolism of the heart is enhanced through intermediaries of TCA cycle, cardiorenal protectivity and caloric restriction. (48)

\section{LIMITATIONS}

There are some limitations that need to be addressed. Firstly, our sample size was calculated using the difference in systolic and diastolic blood pressure and therefore we might have been slightly underpowered to detect a difference in cardiac output between the preoperative and the postoperative measurements. Secondly our sample size was not suited to determine the influence of remission of comorbidities on cardiovascular hemodynamics. Finally, this study is the first to assess hemodynamic changes in bariatric patients and since this is a pilot study we had a short follow-up. Also to further substantiate the findings and generalizability of the device there should be a comparison with echocardiography findings. And if so a control group should also be matched according to gender, age, BMI and body surface are to avoid bias and heterogeneity among the group of patients. 


\subsection{CONCLUSION}

In summary, the aim of this study was to assess the cardiovascular and hemodynamic changes 3 months after bariatric surgery. We have found that compared obese patients prior to bariatric surgery have increased systolic, diastolic and, mean arterial pressure, heart rate and cardiac output that all normalized within three months after bariatric surgery except cardiac output. This suggests that bariatric surgery can have beneficial effects for cardiovascular hemodynamics in the early postoperative phase. 


\subsection{REFERENCES}

1. Ashrafian H, le Roux CW, Darzi A, Athanasiou T. Effects of bariatric surgery on cardiovascular function. Circulation. 2008;118(20):2091-102.

2. Ng M, Fleming T, Robinson M, Thomson B, Graetz N, Margono C, et al. Global, regional, and national prevalence of overweight and obesity in children and adults during 1980-2013: a systematic analysis for the Global Burden of Disease Study 2013. Lancet (London, England). 2014;384(9945):766-81.

3. Buchwald H, Avidor Y, Braunwald E, Jensen MD, Pories W, Fahrbach K, et al. Bariatric surgery: a systematic review and meta-analysis. Jama. 2004;292(14):1724-37.

4. Kasper EK, Hruban RH, Baughman KL. Cardiomyopathy of obesity: a clinicopathologic evaluation of 43 obese patients with heart failure. The American journal of cardiology. 1992;70(9):921-4.

5. Adams JP, Murphy PG. Obesity in anaesthesia and intensive care. British journal of anaesthesia. 2000;85(1):91-108.

6. Aggarwal R, Harling L, Efthimiou E, Darzi A, Athanasiou T, Ashrafian H. The Effects of Bariatric Surgery on Cardiac Structure and Function: a Systematic Review of Cardiac Imaging Outcomes. Obesity surgery. 2015.

7. Pritchett AM, Deswal A, Aguilar D, Foreyt JP, Chan W, Mann DL, et al. Lifestyle Modification with Diet and Exercise in Obese Patients with Heart Failure - A Pilot Study. Journal of obesity \& weight loss therapy. 2012;2(2):1-8.

8. von Elm E, Altman DG, Egger M, Pocock SJ, Gotzsche PC, Vandenbroucke JP. Strengthening the Reporting of Observational Studies in Epidemiology (STROBE) statement: guidelines for reporting observational studies. BMJ (Clinical research ed). 2007;335(7624):806-8.

9. Gizdulich P, Aschero G, Guerissi M, Wesseling KH. Effects of hydrostatic pressure of finger pressure measured with Finapres. Homeostasis. 1995;36:120-9.

10. Gizdulich P, Imholz BP, van den Meiracker AH, Parati G, Wesseling KH. Finapres tracking of systolic pressure and baroreflex sensitivity improved by waveform filtering. Journal of hypertension. 1996;14(2):243-50.

11. Gizdulich P, Prentza A, Wesseling KH. Models of brachial to finger pulse wave distortion and pressure decrement. Cardiovascular research. 1997;33(3):698-705.

12. Wesseling KH. A century of noninvasive arterial pressure measurement: from Marey to Penaz and Finapres. Homeostasis. 1995;36:2-3.

13. Wesseling KH, De Wit B, Van der Hoeven GMA, Van Goudoever J, Settels JJ. Physiocal, calibrating finger vascular physiology for Finapres. Homeostasis. 1995;36:67-82.

14. Westerhof BE, Guelen I, Parati G, Groppelli A, van Montfrans GA, Wieling W, et al. Variable day/ night bias in 24-h non-invasive finger pressure against intrabrachial artery pressure is removed by waveform filtering and level correction. Journal of hypertension. 2002;20(10):1981-6.

15. Vest AR, Heneghan HM, Agarwal S, Schauer PR, Young JB. Bariatric surgery and cardiovascular outcomes: a systematic review. Heart (British Cardiac Society). 2012;98(24):1763-77. 
16. Hsuan CF, Huang CK, Lin JW, Lin LC, Lee TL, Tai CM, et al. The effect of surgical weight reduction on left ventricular structure and function in severe obesity. Obesity (Silver Spring, Md). 2010;18(6):1188-93.

17. Iancu ME, Copaescu C, Serban M, Ginghina C. Favorable changes in arterial elasticity, left ventricular mass, and diastolic function after significant weight loss following laparoscopic sleeve gastrectomy in obese individuals. Obesity surgery. 2014;24(3):364-70.

18. Cunha Lde C, da Cunha CL, de Souza AM, Chiminacio Neto N, Pereira RS, Suplicy HL. Evolutive echocardiographic study of the structural and functional heart alterations in obese individuals after bariatric surgery. Arquivos brasileiros de cardiologia. 2006;87(5):615-22.

19. Karason K, Wallentin I, Larsson B, Sjostrom L. Effects of obesity and weight loss on left ventricular mass and relative wall thickness: survey and intervention study. BMJ (Clinical research ed). 1997;315(7113):912-6.

20. Kokkinos A, Alexiadou K, Liaskos C, Argyrakopoulou G, Balla I, Tentolouris N, et al. Improvement in cardiovascular indices after Roux-en-Y gastric bypass or sleeve gastrectomy for morbid obesity. Obesity surgery. 2013;23(1):31-8.

21. Luaces M, Cachofeiro V, Garcia-Munoz-Najar A, Medina M, Gonzalez N, Cancer E, et al. Anatomical and functional alterations of the heart in morbid obesity. Changes after bariatric surgery. Revista espanola de cardiologia (English ed). 2012;65(1):14-21.

22. Martin J, Bergeron S, Pibarot P, Bastien M, Biertho L, Lescelleur O, et al. Impact of bariatric surgery on $\mathrm{N}$-terminal fragment of the prohormone brain natriuretic peptide and left ventricular diastolic function. The Canadian journal of cardiology. 2013;29(8):969-75.

23. Willens HJ, Chakko SC, Byers P, Chirinos JA, Labrador E, Castrillon JC, et al. Effects of weight loss after gastric bypass on right and left ventricular function assessed by tissue Doppler imaging. The American journal of cardiology. 2005;95(12):1521-4.

24. Alpert MA, Terry BE, Kelly DL. Effect of weight loss on cardiac chamber size, wall thickness and left ventricular function in morbid obesity. The American journal of cardiology. 1985;55(6):783-6.

25. Di Bello V, Santini F, Di Cori A, Pucci A, Talini E, Palagi C, et al. Effects of bariatric surgery on early myocardial alterations in adult severely obese subjects. Cardiology. 2008;109(4):241-8.

26. Cavarretta E, Casella G, Cali B, Dammaro C, Biondi-Zoccai G, Iossa A, et al. Cardiac remodeling in obese patients after laparoscopic sleeve gastrectomy. World journal of surgery. 2013;37(3):56572.

27. Ikonomidis I, Mazarakis A, Papadopoulos C, Patsouras N, Kalfarentzos F, Lekakis J, et al. Weight loss after bariatric surgery improves aortic elastic properties and left ventricular function in individuals with morbid obesity: a 3-year follow-up study. Journal of hypertension. 2007;25(2):43947.

28. Owan T, Avelar E, Morley K, Jiji R, Hall N, Krezowski J, et al. Favorable changes in cardiac geometry and function following gastric bypass surgery: 2-year follow-up in the Utah obesity study. Journal of the American College of Cardiology. 2011;57(6):732-9.

29. Valezi AC, Machado VH. Morphofunctional evaluation of the heart of obese patients before and after bariatric surgery. Obesity surgery. 2011;21(11):1693-7. 
30. Garza CA, Pellikka PA, Somers VK, Sarr MG, Collazo-Clavell ML, Korenfeld Y, et al. Structural and functional changes in left and right ventricles after major weight loss following bariatric surgery for morbid obesity. The American journal of cardiology. 2010;105(4):550-6.

31. Jhaveri RR, Pond KK, Hauser TH, Kissinger KV, Goepfert L, Schneider B, et al. Cardiac remodeling after substantial weight loss: a prospective cardiac magnetic resonance study after bariatric surgery. Surgery for obesity and related diseases : official journal of the American Society for Bariatric Surgery. 2009;5(6):648-52.

32. Kardassis D, Bech-Hanssen O, Schonander M, Sjostrom L, Petzold M, Karason K. Impact of body composition, fat distribution and sustained weight loss on cardiac function in obesity. International journal of cardiology. 2012;159(2):128-33.

33. Koshino Y, Villarraga HR, Somers VK, Miranda WR, Garza CA, Hsiao JF, et al. Changes in myocardial mechanics in patients with obesity following major weight loss after bariatric surgery. Obesity (Silver Spring, Md). 2013;21(6):1111-8.

34. Kanoupakis E, Michaloudis D, Fraidakis O, Parthenakis F, Vardas P, Melissas J. Left ventricular function and cardiopulmonary performance following surgical treatment of morbid obesity. Obesity surgery. 2001;11(5):552-8.

35. Leichman JG, Aguilar D, King TM, Mehta S, Majka C, Scarborough T, et al. Improvements in systemic metabolism, anthropometrics, and left ventricular geometry 3 months after bariatric surgery. Surgery for obesity and related diseases : official journal of the American Society for Bariatric Surgery. 2006;2(6):592-9.

36. Leichman JG, Wilson EB, Scarborough T, Aguilar D, Miller CC, 3rd, Yu S, et al. Dramatic reversal of derangements in muscle metabolism and left ventricular function after bariatric surgery. The American journal of medicine. 2008;121(11):966-73.

37. Maniscalco M, Arciello A, Zedda A, Faraone S, Verde R, Giardiello C, et al. Right ventricular performance in severe obesity. Effect of weight loss. European journal of clinical investigation. 2007;37(4):270-5.

38. Algahim MF, Lux TR, Leichman JG, Boyer AF, Miller CC, 3rd, Laing ST, et al. Progressive regression of left ventricular hypertrophy two years after bariatric surgery. The American journal of medicine. 2010;123(6):549-55.

39. Alpert MA, Lambert CR, Terry BE, Cohen MV, Mulekar M, Massey CV, et al. Effect of weight loss on left ventricular diastolic filling in morbid obesity. The American journal of cardiology. 1995;76(16):1198-201.

40. Dzenkeviciute V, Petrulioniene Z, Sapoka V, Aidietiene S, Abaraviciute L. The effect of weight loss on the cardiac structure and function after laparoscopic adjustable gastric banding surgery in morbidly obese individuals. Obesity surgery. 2014;24(11):1961-8.

41. Rider OJ, Francis JM, Ali MK, Petersen SE, Robinson M, Robson MD, et al. Beneficial cardiovascular effects of bariatric surgical and dietary weight loss in obesity. Journal of the American College of Cardiology. 2009;54(8):718-26.

42. Mukerji R, Petruc M, Fresen JL, Terry BE, Govindarajan G, Alpert MA. Effect of weight loss after bariatric surgery on left ventricular mass and ventricular repolarization in normotensive morbidly obese patients. The American journal of cardiology. 2012;110(3):415-9. 
43. Alpert MA, Nusair MB, Mukerji R, Omran J, Mehra A, Ardhanari S, et al. Effect of weight loss on ventricular repolarization in normotensive severely obese patients with and without heart failure. The American journal of the medical sciences. 2015;349(1):17-23.

44. Karason K, Wallentin I, Larsson B, Sjostrom L. Effects of obesity and weight loss on cardiac function and valvular performance. Obesity research. 1998;6(6):422-9.

45. Lin CH, Kurup S, Herrero P, Schechtman KB, Eagon JC, Klein S, et al. Myocardial oxygen consumption change predicts left ventricular relaxation improvement in obese humans after weight loss. Obesity (Silver Spring, Md). 2011;19(9):1804-12.

46. Ashrafian H, Athanasiou T, le Roux CW. Heart remodelling and obesity: the complexities and variation of cardiac geometry. Heart (British Cardiac Society). 2011;97(3):171-2.

47. Chatelain P, Robberecht P, de Neef P, Claeys M, Christophe J. Low responsiveness of cardiac adenylate cyclase activity to peptide hormones in spontaneously hypertensive rats. FEBS letters. 1979;107(1):86-90.

48. Fenske W, Athanasiou T, Harling L, Drechsler C, Darzi A, Ashrafian H. Obesity-related cardiorenal disease: the benefits of bariatric surgery. Nature reviews Nephrology. 2013;9(9):53951. 



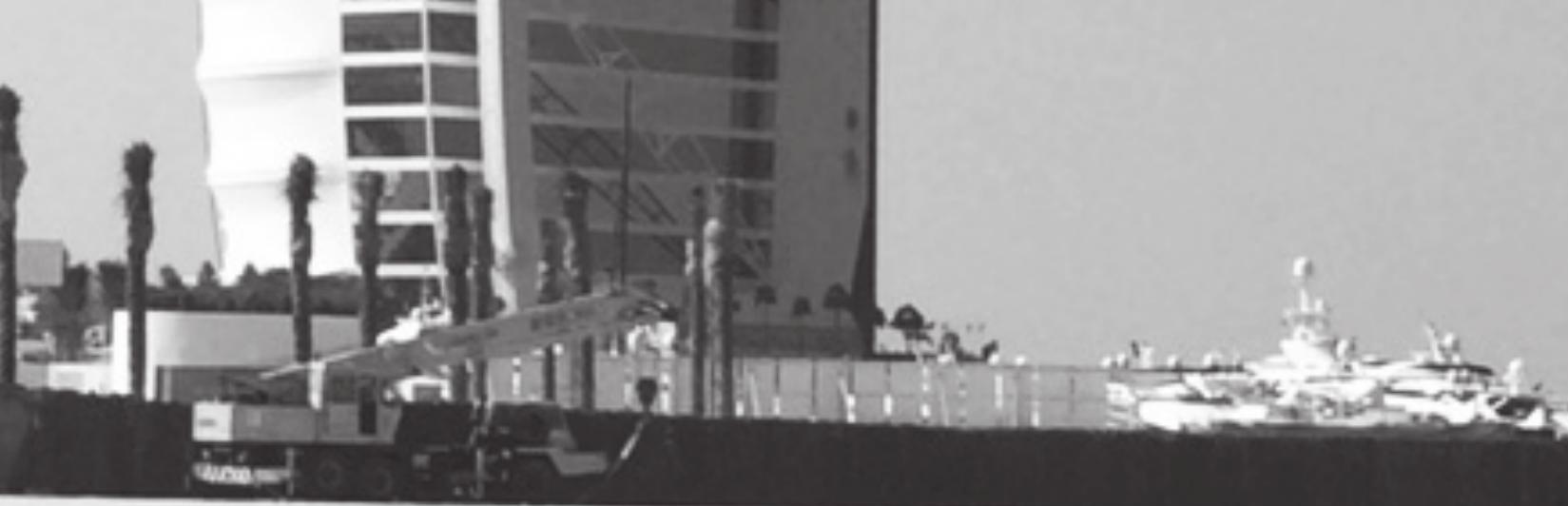




\title{
5. PERIOPERATIVE RESPIRATORY CARE IN OBESE PATIENTS UNDERGOING BARIATRIC SURGERY: IMPLICATIONS FOR CLINICAL PRACTICE
}

\author{
Sjaak Pouwels ${ }^{1,2}$ \\ Frank W.J.M. Smeenk ${ }^{4,5}$ \\ Loes Manschot ${ }^{3}$ \\ Bianca Lascaris ${ }^{3}$ \\ Simon Nienhuijs ${ }^{1}$ \\ R. Arthur Bouwman ${ }^{3}$ \\ Marc P. Buise ${ }^{3}$
}

Respir Med. 2016 Aug;117:73-80

1 Department of Surgery, Catharina Hospital, Eindhoven, The Netherlands

2 Department of Epidemiology, CAPHRI Research School, Maastricht University, The Netherlands

3 Department of Anaesthesiology, Intensive Care and Pain Medicine Catharina Hospital, Eindhoven, The Netherlands 4 Department of Respiratory Medicine, Catharina Hospital, Eindhoven, The Netherlands 5 SHE School of Health Professions Education, Maastricht University, The Netherland 


\section{ABSTRACT}

Obesity is an increasing problem worldwide. The number of people with obesity doubled since the 1980's to affect an estimated 671 million people worldwide. Obese patients in general have an altered respiratory physiology and can have an impaired lung function, which leads to an increased risk of developing pulmonary complications during anaesthesia and after bariatric surgery (approximately $8 \%$ ). Therefore the respiratory management of the bariatric surgical patient provides a number of challenges. This review will focus on the perioperative respiratory care in bariatric surgical patients discussing respiratory physiology in the obese and perioperative respiratory care in bariatric surgery. Finally the value of preoperative pulmonary function testing and preoperative OSAS screening will be discussed. 


\subsection{INTRODUCTION}

Obesity is an increasing problem worldwide. The number of people with obesity doubled since the 1980's to affect an estimated 671 million people worldwide. (1, 2) Among these, the number of people with the highest Body Mass Index $\left(B M I>40 \mathrm{~kg} / \mathrm{m}^{2}\right)$ grew twice as fast as the group of people with a BMI of $30-40 \mathrm{~kg} / \mathrm{m}^{2}$. $(2,3)$ In the Netherlands, $48.3 \%$ of the people aged 19 years and older have overweight (defined as a BMI $>25 \mathrm{~kg} / \mathrm{m}^{2}$ ). (2)

The only treatment with a longstanding effect is bariatric surgery. (4) With the increasing prevalence of obesity, the worldwide numbers of bariatric surgical procedures are also increasing. (4) With obesity affecting many organ systems, under which the respiratory system, it is therefore not surprising that the respiratory management of obese subjects undergoing bariatric surgery represents a growing challenge.

This review will focus on the perioperative respiratory care in bariatric surgical patients discussing the following subjects:

- $\quad$ Respiratory physiology in the obese patients

- $\quad$ Perioperative respiratory care in bariatric surgery

- $\quad$ The value of preoperative pulmonary function testing and OSAS screening

\subsection{RESPIRATORY PHYSIOLOGY IN THE OBESE}

Obesity negatively affects many organ systems, including the respiratory system. It is associated with an altered lung function, characterised by a reduction of lung volumes, mostly a restrictive pattern. The pathogenesis behind this is multifactorial, but an increased truncal fat load is one of the possible mechanisms. (5) A restrictive pattern is seen when both the vital capacity (VC) and the total lung capacity (TLC) is below $80 \%$ of their predicted value and the Tiffeneau index (forced expiratory volume in 1 second (FEV1)/VC) is $\geq 0.7$.

Because of affected respiratory physiological parameters such as compliance, neuromuscular strength, work of breathing (WOB), lung volumes and spirometric measurements $(6,7)$, obese subjects are prone to develop pulmonary complications after bariatric surgery.

\section{RELATIONSHIP BETWEEN ANTHROPOMETRIC VARIABLES AND LUNG FUNCTION}

Soriano et al (8) investigated over 3000 people in Spain and found a restrictive pattern in 12.7\%. A higher BMI $\left(>30 \mathrm{~kg} / \mathrm{m}^{2}\right)$ was independently associated with a restrictive spirometry. 
(8) This was confirmed by Mannino et al. (9) who measured spirometry in different locations around the world. He found that a BMI below or above the reference categories (18.5-24 kg.m2) was a significant risk factor for a restrictive pattern. (9) Less is known about the prevalence of a restrictive lung function among obese people. Most research was done with obese people who were a candidate for bariatric surgery. Groups were small, varying between 20-150 patients and the prevalence varied between 6-50\%. (10-13)

The impact of obesity on the respiratory system may vary from patient to patient and cannot be predicted from weight and/or BMI measurements alone. While assessment of BMI gives an impression about overall nutritional status, it does not differentiate lean mass, fat mass and the distribution of adipose tissue. This distinction might be important as lean- and fat mass have an opposite effect on lung function and the distribution of fat seems more relevant than total body fat per se. Body fat percentage was associated with decreased FVC, FEV1 ratio and FEV1/VC ratios in men and women respectively. (14-18) Moreover, others showed an adverse relation with waist to hip ratios and the respiratory system. High waist to hip ratios were associated with reduced lung function and poorer gas-exchange $(15,16,18,19)$

Most studies use spirometry to assess lung function. Babb et al. (19) took a different approach. They state that the End-Expiratory Lung Volume (EELV or residual volume (RV)) is very sensitive to changes in static compliance of the lung and chest wall. Deposits of fat on the chest wall thus specifically alter the EELV. (19) They measured absolute and relative fat mass with MRI and compared it to the End-Expiratory Lung Volume (EELV). They found that fat distribution is relatively similar between lean and obese men and women, and that therefore the increase in chest wall fat distribution is proportional to the increase in obesity. (19) This means that measurements of overall obesity are significantly related to lung function as measured with EELV. So far, the books are not yet closed on which measurement should be used to assess the effect of obesity on lung function, but a combination of Waist/Hip (W/H) ratio and BMI seems recommended.

\section{RESPIRATORY COMPLIANCE}

Respiratory compliance is the ability of the respiratory system to stretch during a change in volume relative to an applied change in pressure. (6) Total respiratory compliance (e.g. the compliance of the pulmonary and extrapulmonary structures in the thoracic cage) can be reduced in obesity and in patients with the obesity-hypoventilation syndrome to as little as one-third of the normal values. $(6,20)$ This is mainly the result of reduced distensibility of extrapulmonary structures due to excess truncal fat. $(6,20)$ Secondly, the increase in pulmonary blood volume and increased closure of dependant airways may also contribute to the low lung compliance seen in obese people. (21) These physiological changes are even 
more pronounced during recumbency in obese subjects (as compared to normal weight subjects), due to increased gravitational effects of the abdomen. (22)

\section{LUNG VOLUMES}

The most consistent indicator of obesity is a reduction in expiratory reserve volume (ERV). This is due to a displacement of the diaphragm more cranially into the thoracic cage by the 'obese' abdomen and the increased chest wall mass. $(6,23,24)$ This association is seen in modest obesity, but the ERV rapidly decreases with increasing BMI. $(6,25,26)$ The ERV decreases because the 'obese' abdomen inhibits the diaphragm to extend in caudally.

On the other hand, obesity has modest effects on residual volume (RV) and total lung capacity (TLC), but a relatively larger effect in reducing functional residual capacity (FRC). (26-28) In some studies the reduction of the FRC is so marked that it approaches RV. (27) When the reduced FRC is equal to or lower than the closing volume, thoracic gas trapping may take place in obese subjects, as indicated by an elevated RV/TLC ratio. (28-30) The effects of obesity on lung volumes are shown in figure 5.1 .

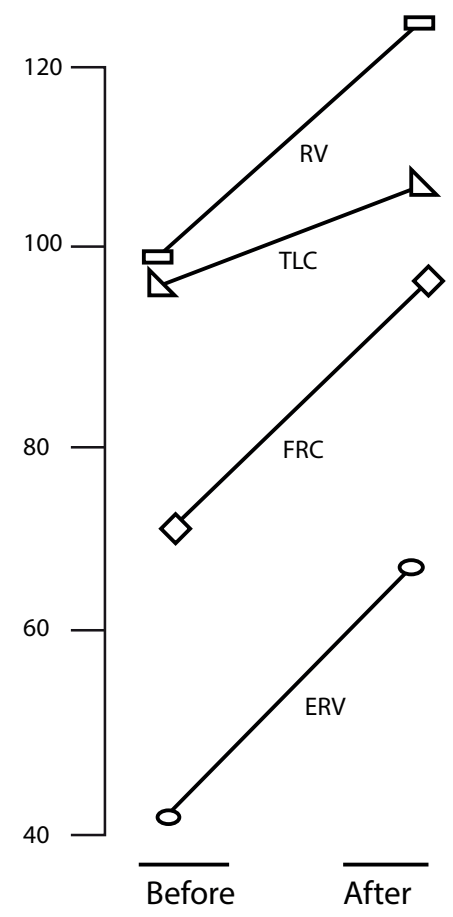

FIGURE 5.1: Lung function parameters before and after surgical weight loss (Adapted from Thomas et al. (93)) Abbreviations: ERV $=$ Expiratory Reserve Volume, FRC $=$ Functional Residual Capacity, TLC $=$ Total Lung Capacity, RV = Residual Volume 


\section{WORK OF BREATHING}

To compensate the reduced respiratory compliance, (severely) obese subjects may breathe rapidly and shallowly, to maintain eucapnia. $(6,23,31-33)$ Therefore people with obesity have a higher Work of Breathing (WOB). Because of a restrictive pattern, breathing takes place at a less compliant part of the pressure-volume curve. Thus they can develop a rapid, shallow breathing pattern, which comes with a higher oxygen cost. $(6,23,24)$ The oxygen cost of breathing is an index that represents the energy required to breathe. (21) This index shows the oxygen consumed by the respiratory muscles per litre of ventilation. (21) Kress et al. (34) investigated eighteen severely obese patients and found a $16 \%$ reduction in oxygen consumption after elective intubation, mechanical ventilation and anaesthesia from the baseline values, as compared to $<1 \%$ reduction among controls. This (relative) respiratory inefficiency among the obese suggests a decreased ventilatory reserve and a (possible) predisposition to respiratory failure. $(6,23,24,34)$

\section{RESPIRATORY MUSCLE STRENGTH}

Regarding respiratory muscle strength, different results are being reported in the current literature. Several studies indicate that obese people have greater risk of developing respiratory muscle inefficiency, in terms of less capability to generate normal maximal inspiratory pressure and expiratory pressures. (6) Other studies showed that there was no difference in inspiratory pressures between obese individuals and non-obese controls. (35-37) A possible explanation for impaired respiratory muscle function in obesity can be the increased elastic load that the respiratory muscles are required to overcome during inspiration. $(6,38)$

A mechanical disadvantage exists because of an overstretched diaphragm, leading to decreased inspiratory muscle strength and efficiency. $(6,39)$ Also some studies indicate decreased levels of skeletal muscle glycogen synthase activity in obese subjects that can be a contributing factor in the decrease in isokinetic skeletal muscle endurance. $(40,41)$

\section{CLINICAL RELEVANCE OF PULMONARY FUNCTION CHANGES}

The pulmonary function changes due to obesity can roughly be summarised in three parts I) a restrictive effect of mass on the chest wall, II) a tendency to breathe at low lung volumes and III) the effect of fat distribution on pleural pressure. $(28,42-47)$ The physiological changes are heightened during sleep in supine position, due to a negative impact pulmonary mechanics of diaphragm impedance by the abdomen with a change in lung volume. This leads to intolerability for apnoeic episodes and to early desaturation. These pulmonary mechanics changes in obesity lead to a lower FRC, FVC and FEV ${ }_{1}$. $\left.28,42-47\right)$ 


\section{OBSTRUCTIVE SLEEP APNOEA SYNDROME (OSAS) AND OBESITY HYPOVENTILATION SYNDROME (OHS)}

Research in patients with the Obesity Hypoventilation Syndrome (OHS: the existence of alveolar hypoventilation which cannot be explained by an other neuromuscular or pulmonary disorder) demonstrated that these patients exhibit a lowered neuromuscular respons to hypercapnia and hypoxemia, caused by a blunted central drive. $(6,23,31-33)$ It is unclear, however, what causes this blunted central drive. A lowered chemosensitivity could be genetic, but other factors such as a distorted sleep-breathing pattern (Obstructive Sleep Apnoea Syndrome (OSAS)) and neuro-hormonal factors could also play a role. The fact that effective treatment of the obstructed breathing may partly correct nocturnal hypoxemia and awake hypercapnia, seems to prove that upper airway obstruction and flow limitation are important factors in the development of OHS. $(6,23,24)$

But few patients, even among those with severe OSAS, develop daytime hypercapnia. Possibly, the severity of the nocturnal hypoxemia is important. $(6,26,31-33)$ Despite a similar BMI, severity of the apnoea/hypopnea index, arousal indices and sleep architecture, OHS patients exhibit a more severe desaturation compared to the eucapnic obese group (23).

TABLE 5.1. Correlation between anthropometric variables and pulmonary function parameters

\begin{tabular}{|l|c|c|c|c|}
\hline Jung et al. (14) & Gender & $\begin{array}{c}\text { Anthropometric } \\
\text { variable }\end{array}$ & $\begin{array}{c}\text { Pulmonary function } \\
\text { variable }\end{array}$ & Correlation \\
\hline Zavorsky et al. (15, 16) & $\begin{array}{c}\text { Men } \\
\text { Women }\end{array}$ & $\begin{array}{c}\text { Body fat percentage } \\
\text { Body fat percentage }\end{array}$ & $\begin{array}{c}\text { FVC and FEV } \\
\text { FEV } / \text { VC }\end{array}$ & $\begin{array}{c}\text { Negative } \\
\text { Negative }\end{array}$ \\
\hline Wehrmeister et al. (17) & Men & Waist/hip ratio & $\begin{array}{c}\text { Gas exchange } \\
\text { Gas exchange }\end{array}$ & $?$ \\
\hline Rossi et al. (18) & $\begin{array}{c}\text { Men } \\
\text { Woman }\end{array}$ & $\begin{array}{c}\text { Body fat percentage } \\
\text { Body fat percentage }\end{array}$ & $\begin{array}{c}\text { FVC and FEV } \\
\text { FVC and FEV }\end{array}$ & $\begin{array}{c}\text { Inverse } \\
\text { Inverse }\end{array}$ \\
\hline Babb et al. (19) & $\begin{array}{c}\text { Men } \\
\text { Women }\end{array}$ & $\begin{array}{c}\text { Fat deposition thorax } \\
\text { Fat deposition thorax }\end{array}$ & $\begin{array}{c}\text { RV } \\
\text { RV }\end{array}$ & $\begin{array}{l}\text { Inverse } \\
\text { Inverse }\end{array}$ \\
\hline
\end{tabular}

Abbreviations: $\mathrm{FVC}=$ Forced Vital Capacity, $\mathrm{FEV}_{1}=$ Forced expiratory volume in 1 second, $\mathrm{RV}=$ Residual Volume

Animal research showed that nocturnal hypoxemia lowers the hypoxic ventilatory drive and raises the arousal threshold, possibly because of an effect on the synthesis and turnover of neurotransmitters. $(23,48-51)$ Because of this, patients fail to compensate adequately after an episode of hypoventilation. This causes decreased ventilation in between episodes for a given CO2-load, combined with a relatively shorter time-span between episodes considering the duration of apnoeic episodes. $(48,50,51)$ Norman et al. (52) hypothesizes that repeated nocturnal CO2-accumulations cause bicarbonate retention by the kidneys. Most patients 
have enough time to compensate for this brief raise in $\mathrm{CO} 2$. But if patients compensate insufficiently after an event, chronic bicarbonate retention develops, raising the threshold for hypercapnia. $(23,52)$ What causes this diminished response is yet to be discovered. Possible actors might be leptin and IL-GF-1 because of their stimulating effect on central respiratory centres. (23)

\subsection{PERIOPERATIVE RESPIRATORY CARE IN BARIATRIC SURGERY}

Because the percentage of obese people in the population increases $(2,3)$, more people are at risk for an altered lung function due to obesity. As a consequence, the risk of respiratory complications under general anaesthesia might increase as well. This can result in hypoxemia, hypercapnia and increased formation of atelectasis.

\section{INDUCTION AND AIRWAY MANAGEMENT}

Obesity increases the risk of a difficult airway during induction, because of an altered upper airway anatomy. $(6,7,23)$ Heinrich et al. $(53)$ found that $6 \%$ of cases of a difficult laryngoscopy over a 6 -year period occurred in patients with a BMI $\geq 35 \mathrm{~kg} / \mathrm{m}^{2}$. The incidence of a difficult intubation is increased from $5.8 \%$ in the general population and $15.8 \%$ in people with a BMI $>30 \mathrm{~kg} / \mathrm{m}^{2}$. (54) The restricted lung function leads to a greater risk of desaturation during induction. The lowered FRC can approach the closing capacity, which can lead to closing of airways during tidal breathing. $(6,54,55)$ This leads to shunting and a ventilation/perfusion mismatch. This is exaggerated by a supine position, in which the abdominal pressure and the pulmonal blood volume are increased and decruitment of dependent alveoli exists, and by an increased oxygen demand. (55)

Techniques that can be used to reduce this risk are:

- Prediction difficult intubation: indirect mirror laryngoscopy. (56)

\section{- (Awake) videolaryngoscopy:}

Moore et al. (57) found that $96 \%$ of 50 morbid obese patients undergoing bariatric surgery and classified as having a difficult airway were successfully intubated using awake videolaryngoscopy.

\section{- Head-up induction:}

Several studies have investigated different positioning techniques to improve the laryngeal view in patients with morbid obesity. Lee et al. (58) found that the laryngeal view can be significantly improved when patients were put in a 25 degrees head-up position when 
compared with the conventional supine position. Collins et al. (59) found a statistical significant better laryngeal view when morbidly obese patients were placed in the 'ramped' position, in which the external auditory meatus was at the same level as the sternal notch. Gupta et al. (60) compared rapid sequence induction (RSI) in semi-erect position with the investigator in front of the patient compared to RSI in supine position with the GlideScope videolaryngoscope of morbidly obese patients, but found no significant differences between the two groups when it comes to intubation parameters or patient safety.

\section{- Pre-oxygenation:}

Dixon et al. (61) found obesity achieved higher oxygen tensions and therefore a clinically significant increase in the desaturation safety period in the 25 degrees head-up position. Ramkumar et al. (62) investigated the 20 degrees head up pre-oxygenation in non-obese individuals, and found that time to desaturation (<93\%) was significantly prolonged as well.

\section{- Apneic oxygenation:}

Ramachandran et al. (63) found that providing obese patients with additional nasal O2 during simulated difficult laryngoscopy was associated with a significant prolongation of $\mathrm{SpO} 2 \geq$ $95 \%$, a significant increase in patients with $\mathrm{SpO} 2 \geq 95 \%$ apnoea at $6 \mathrm{~min}$, and a significantly higher minimum SpO2. Resaturation times were no different.

\section{VENTILATION StRATEGIES IN OBESE PATIENTS}

General anaesthesia and paralysis negatively affect pulmonary gas exchange and respiratory mechanics (figure 5.2). When PEEP is not applied, atelectasis formation is present in $90 \%$ of patients under general anaesthesia. $(64,65)$

Patients with morbid obesity have a higher risk of atelectasis formation, exhibit more profound changes in respiratory function and are at a higher risk for hypoxemia. $(6,7,66)$ Pelosi et al. (66) found that under general anaesthesia, $\mathrm{PaO}_{2}$ is inversely related to BMI. The severity of atelectasis formation is related to body weight and extends longer in the postoperative phase when patients are severely overweight. $(67,68)$

Reinius and colleagues (68) showed in obese patients under general anaesthesia that PEEP in combination with recruitment manoeuvres reduced atelectasis and improved $\mathrm{PaO} 2 / \mathrm{FiO} 2$ ratio. This effect was still present 40 minutes later. The compliance was also improved. (68) Aldenkortt et al. (69) found similar results based on their performed meta-analysis on the effects of different ventilation strategies in obese patients. Also Aldenkortt et al. (69) compared pressure- and volume-controlled ventilation (PCV and VCV). In terms of intraoperative $\mathrm{PaO}_{2}, \mathrm{FiO}_{2}$ ratio and tidal volumes no significant differences were found between the both ventilation strategies. (69) 


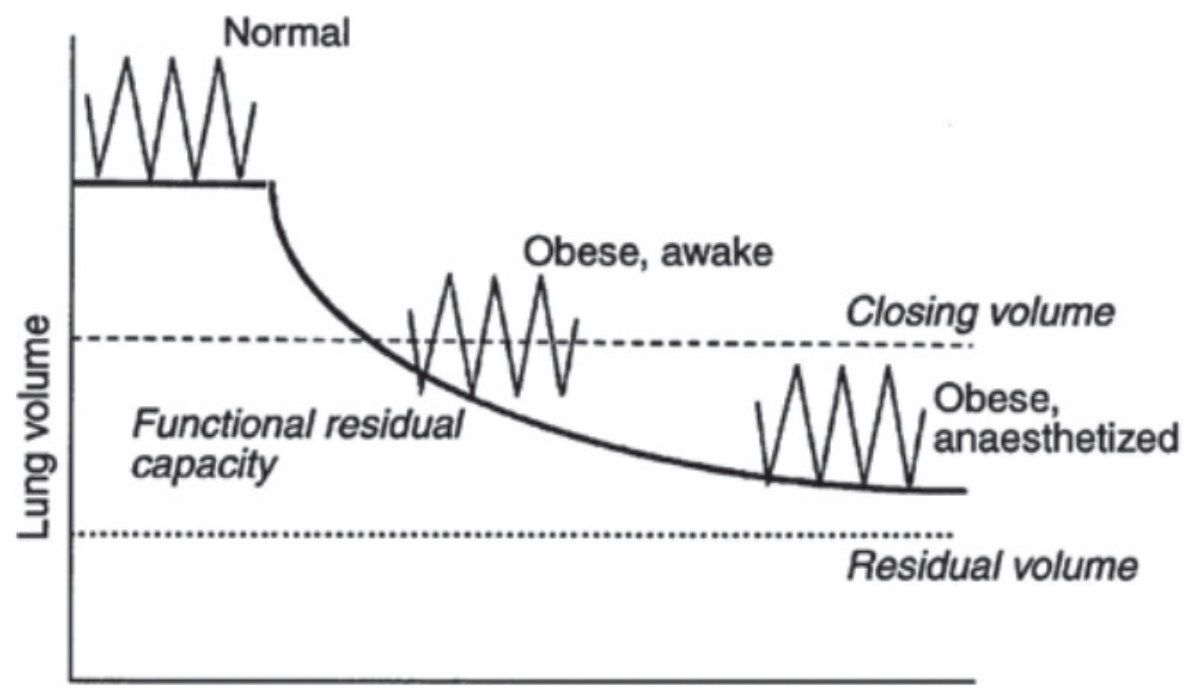

FIGURE 5.2: Effects of anaesthesia on the functional residual capacity of the obese (30)

\section{POSITIVE AIRWAY PRESSURE (BIPAP)}

Yu et al. (70) hypothesized that biPAP ventilation may diminish the development of pulmonary shunt and may improve ventilation-perfusion mismatch when compared to standard IPPV, with or without PEEP when neuromuscular paralysis has been used during surgery. They found that biPAP ventilation was beneficial in decreasing ventilation-perfusion mismatch and improving oxygenation when compared with conventional IPPV (with or without PEEP) (70)

Ebeo et al. (71) investigated the effect of BiPAP on pulmonary function in obese patients following open gastric bypass surgery. This resulted in significantly higher $\mathrm{VC}$ and $\mathrm{FEV}_{1} 12$ 24 hours postoperative, but did not result in fewer hospital days or lower complication rates. (71) Joris et al. (72) found similar results in terms of postoperative pulmonary function, but no significant differences were found between the BiPAP and control group in terms of peak expiratory flow rate. (72)

\section{NON-INVASIVE VENTILATION STRATEGIES (CPAP AND NIV)}

Continuous positive airway pressure (CPAP) and NIV have been used to prevent and treat acute respiratory failure after surgery or to treat acute respiratory failure. $(73,74)$ What the most ideal ventilation strategy is to reduce postoperative morbidity is unclear. A meta-analysis (75) showed that CPAP following abdominal surgery significantly reduced postoperative 
pulmonary complications, atelectasis and pneumonia, which is in contrast with a recent Cochrane review (76) that stated the evidence is of low quality.

The feasibility and safety of NIV use in the recovery room after various types of surgery has been demonstrated (77) and also a recent meta-analysis (78) showed that in perioperative bariatric care NIV is well tolerated and significantly reduces respiratory complications. However there is still lack of comparative studies to determine which ventilation strategy is superior in perioperative bariatric care.

\section{INFLUENCE OF METABOLIC SYNDROME AND COMORBIDITIES ON THE PERIOPERATIVE PERIOD}

Bariatric surgery is an acceptable and effective method to manage obesity-related comorbidities in morbidly obese patients. $(79,80)$ Nearly four in five patients scheduled for bariatric surgery has metabolic syndrome. (80) In particular the presence of cardiac, pulmonary, metabolic and hepatic comorbidities may vary in patients scheduled for bariatric surgery, thus posing particular challenges to the anaesthesiologist. (79) Hypertension (both systemic and pulmonary), dyslipidaemia and hyperglycaemia respond to bariatric surgery. (79-81) A large retrospective study by Purnell et al. (80) showed that there was no significant difference in perioperative complications in patients with our without metabolic syndrome.

\subsection{IS PREOPERATIVE PULMONARY FUNCTION TESTING NECESSARY IN BARIATRIC SURGERY?}

The current body of literature is sparse regarding the effects of an impaired pulmonary function and its relation with the occurrence postoperative complications in bariatric surgery. A study conducted by van Huisstede et al. (82) investigated the relationship between pulmonary function parameters and the risk of postoperative complications in a 485 patients. They found 53 complications of which 8 of them were from pulmonary origin. Patients with complications had a significantly lower $\mathrm{FEV}_{1}$ (mean $86.9 \%$ of predicted) and FVC (mean 95.6\% of predicted) compared to patients without complications $(\mathrm{P}<0.05)$. (82) $\mathrm{A} \mathrm{FEV}_{1} / \mathrm{FVC}$ $<70 \%$ and a $\delta \mathrm{FEV} 1 \geq 12 \%$ were found to be predictors for pulmonary complications. (82) In the study by Sood et al. (83) morbidly obese patients (with a BMI $>40 \mathrm{~kg} / \mathrm{m}^{2}$ ) have increased odds of developing pulmonary complications

Questions arise whether it might be useful to perform preoperative pulmonary function testing in patients scheduled for bariatric surgery. And which BMI group is at risk for postoperative (pulmonary) complications and does this increased risk has a relation with the 
lung function. A second problem that may arise is the possible need for screening for OSAS, in this population. Because this co morbidity occurs frequently and if untreated, it might result in postoperative complications. (84-87)

Lastly, we searched the literature for evidence for preoperative strategies that might be applied in obese patients with impaired pulmonary function to prevent postoperative complications.

\section{PREOPERATIVE PULMONARY FUNCTION SCREENING}

The current body of literature regarding the clinical utility of preoperative pulmonary assessment in bariatric surgery remains questionable. Reasons to preoperatively screen patients is to identify those who are at high risk of developing postoperative pulmonary complications. To assess the utility and predictive value we must separate the literature regarding open and laparoscopic bariatric procedures.

Farina et al. (12) investigated the value of spirometry as a preoperative screening tool to identify patients scheduled for open bileopancreatic diversion at risk for postoperative pulmonary complications (PPC). They found a very low rate of PPC's (7.5\%) in patients with suspected restrictive pulmonary impairment. (12) Hamoui et al. (88) investigated the usefulness of pulmonary function tests in predicting the overall risk for complications (including PPC's). They found on multivariate analysis that age $(\mathrm{p}=0.01)$ and a decreased VC $(\mathrm{p}=0.0007)$ were significant predictors for postoperative pulmonary complications. (88)

Catheline et al (10) screened 77 patients prior to bariatric surgery on cardiac and pulmonary abnormalities and found no consequences for the management of the perioperative period. Notwithstanding their results they still found based on their clinical experience that cardiac and pulmonary screening essential prior to bariatric surgery. (10)

\section{PREOPERATIVE OSAS SCREENING}

Nepomnayshy et al. (89) investigated the additive value of screening for sleep apnoea prior to laparoscopic bariatric surgery for predicting postoperative pulmonary complications and compared them with obese patients undergoing orthopaedic surgical procedures. As a result of screening, of 882 patients, 119 bariatric patients (25\%) were newly diagnosed with OSAS. The orthopaedic surgery group had $17.3 \%$ (72 of 415 patients) with pre-existing OSAS. The unscreened orthopaedic patients had complication rate of $6.7 \%$ compared to $2.6 \%$ for the screened bariatric patients. This difference was not statistically significant after adjusting for age and comorbidity ( $\mathrm{p}=0.3383)$. (89) 
Peromaa-Haavisto et al. (90) showed that in a population of 197 obese patients scheduled for bariatric surgery, there was a prevalence of OSAS was $71 \%$, with a significantly higher prevalence in males (90\%) compared with women (60\%). According to their study results, they recommend OSAS screening preoperatively especially in obese men. (90)

\section{PREOPERATIVE PULMONARY PREPARATION}

The current evidence around preoperative pulmonary preparation (in case of an impaired lung function found during screening) is lacking. Also the effects on postoperative complications are not clear.

In a study by Barbalho-Moulim et al. (91) randomised 32 obese women undergoing elective open bariatric surgery to either an inspiratory muscle-training group or usual care group. Compared to the preoperative values, the MIP decreased significantly in both groups after surgery. However the reduction in MIP was $28 \%$ in the inspiratory muscle training group en $47 \%$ in the usual care group. (91) There was a significant reduction in postoperative complications in the inspiratory muscle-training group.

Van Huisstede et al. (82) concluded that the risk of pulmonary complications after laparoscopic bariatric surgery is low. However patients with abnormal spirometry test results have a threefold risk of complications after laparoscopic bariatric surgery. (82)

In obese patients with asthma, van Huisstede et al. (92) recently showed that bariatric surgery has beneficial effects on lung function, in terms of small airway function, decreased systemic inflammation and the number of mast cells in the airways compared to obese patients without asthma. In both groups $\mathrm{FEV}_{1}, \mathrm{FVC}$ and TLC significantly improved, whereas $\mathrm{FEV}_{1} / \mathrm{VC}$ only improved in the obese patients with asthma. (92) Unfortunately it is unclear whether obesity/ morbid obesity and preoperative asthma control has influence on the postoperative outcomes.

It can be hypothesized that perioperative respiratory physiotherapy (in bariatric surgery) might have a role in preventing PPC's in patients with a (obesity induced) respiratory defect. Also for specific groups (asthma, OSA and OHS patients) pulmonary function tests may be useful and might bring clinical advantages. 


\subsection{CONCLUSION}

The majority of the obese patients have an altered respiratory physiology and have an impaired lung function, which leads to an increased risk of developing pulmonary complications during anaesthesia and after bariatric surgery. Therefore the respiratory management of the bariatric surgical patient is challenging. There are a growing number of studies in particular around optimal ventilation strategies to minimize the risk of postoperative complications. Unfortunately, this is still a grey area because not one ventilation strategy has shown superiority in preventing postoperative atelectasis after bariatric surgery. Whether patients scheduled for bariatric surgery need to be screened for obesity related pulmonary function impairment (and OHS/OSAS) is still subject to discussion. 


\subsection{REFERENCES}

1. Obesity: preventing and managing the global epidemic. Report of a WHO consultation. World Health Organization technical report series. 2000;894:i-xii, 1-253.

2. Ng M, Fleming T, Robinson M, Thomson B, Graetz N, Margono C, et al. Global, regional, and national prevalence of overweight and obesity in children and adults during 1980-2013: a systematic analysis for the Global Burden of Disease Study 2013. Lancet. 2014;384(9945):766-81.

3. Sturm R. Increases in morbid obesity in the USA: 2000-2005. Public health. 2007;121(7):492-6.

4. Angrisani L, Santonicola A, Iovino P, Formisano G, Buchwald H, Scopinaro N. Bariatric Surgery Worldwide 2013. Obesity surgery. 2015;25(10):1822-32.

5. Watson RA, Pride NB, Thomas EL, Ind PW, Bell JD. Relation between trunk fat volume and reduction of total lung capacity in obese men. Journal of applied physiology (Bethesda, Md : 1985). 2012;112(1):118-26.

6. Sood A. Altered resting and exercise respiratory physiology in obesity. Clinics in chest medicine. 2009;30(3):445-54, vii.

7. Pedoto A. Lung physiology and obesity: anesthetic implications for thoracic procedures. Anesthesiology research and practice. 2012;2012:154208.

8. Soriano JB, Miravitlles M, Garcia-Rio F, Munoz L, Sanchez G, Sobradillo V, et al. Spirometricallydefined restrictive ventilatory defect: population variability and individual determinants. Primary care respiratory journal : journal of the General Practice Airways Group. 2012;21(2):187-93.

9. Mannino DM, McBurnie MA, Tan W, Kocabas A, Anto J, Vollmer WM, et al. Restricted spirometry in the Burden of Lung Disease Study. The international journal of tuberculosis and lung disease : the official journal of the International Union against Tuberculosis and Lung Disease. 2012;16(10):1405-11.

10. Catheline JM, Bihan H, Le Quang T, Sadoun D, Charniot JC, Onnen I, et al. Preoperative cardiac and pulmonary assessment in bariatric surgery. Obesity surgery. 2008;18(3):271-7.

11. Saliman JA, Benditt JO, Flum DR, Oelschlager BK, Dellinger EP, Goss CH. Pulmonary function in the morbidly obese. Surgery for obesity and related diseases : official journal of the American Society for Bariatric Surgery. 2008;4(5):632-9; discussion 9.

12. Farina A, Crimi E, Accogli S, Camerini G, Adami GF. Preoperative assessment of respiratory function in severely obese patients undergoing biliopancreatic diversion. European surgical research Europaische chirurgische Forschung Recherches chirurgicales europeennes. 2012;48(2):106-10.

13. Wei YF, Wu HD, Chang CY, Huang CK, Tai CM, Hung CM, et al. The impact of various anthropometric measurements of obesity on pulmonary function in candidates for surgery. Obesity surgery. 2010;20(5):589-94.

14. Jung DH, Shim JY, Ahn HY, Lee HR, Lee JH, Lee YJ. Relationship of body composition and C-reactive protein with pulmonary function. Resp Med. 2010;104:1197-203.

15. Zavorsky GS, Kim DJ, Sylvestre JL, Christou NV. Alveolar-membrane diffusing capacity improves in the morbidly obese after bariatric surgery. Obesity surgery. 2008;18(2):256-63. 
16. Zavorsky GS, Murias JS, Kim DJ, Gow J, Sylvestre JL, Christou NV. Waist-to-hip ratio is associated with pulmonary gas exhange in the morbidly obese. Chest. 2007;131(2):362-7.

17. Wehrmeister FC, Menezes AM, Muniz LC, Martinez-Mesa J, Domingues MR, Horta BL. Waist circumference and pulmonary function: a systematic review and meta-analysis. Systematic reviews. 2012;1:55.

18. Lamont LS, Romito R, Rossi K. Fat-free mass and gender influences the rapid-phase excess postexercise oxygen consumption. Applied physiology, nutrition, and metabolism = Physiologie appliquee, nutrition et metabolisme. 2010;35(1):23-6.

19. Babb TG, Wyrick BL, DeLorey DS, Chase PJ, Feng MY. Fat distribution and end-expiratory lung volume in lean and obese men and women. Chest. 2008;134(4):704-11.

20. Naimark A, Cherniack RM. Compliance of the respiratory system and its components in health and obesity. Journal of applied physiology. 1960;15:377-82.

21. Rochester DF, Enson Y. Current concepts in the pathogenesis of the obesity-hypoventilation syndrome. Mechanical and circulatory factors. The American journal of medicine. 1974;57(3):40220.

22. Koenig SM. Pulmonary complications of obesity. Am J Med Sci. 2001;321(4):249-79.

23. Lin CK, Lin CC. Work of breathing and respiratory drive in obesity. Respirology (Carlton, Vic). 2012;17(3):402-11.

24. Piper AJ, Grunstein RR. Big breathing: the complex interaction of obesity, hypoventilation, weight loss, and respiratory function. Journal of applied physiology (Bethesda, Md : 1985). 2010;108(1):199-205.

25. Sutherland TJ, Goulding A, Grant AM, Cowan JO, Williamson A, Williams SM, et al. The effect of adiposity measured by dual-energy X-ray absorptiometry on lung function. The European respiratory journal. 2008;32(1):85-91.

26. Jenkins SC, Moxham J. The effects of mild obesity on lung function. Respir Med. 1991;85(4):30911.

27. Gibson GJ. Obesity, respiratory function and breathlessness. Thorax. 2000;55 Suppl 1:S41-4.

28. Rubinstein I, Zamel N, DuBarry L, Hoffstein V. Airflow limitation in morbidly obese, nonsmoking men. Annals of internal medicine. 1990;112(11):828-32.

29. Douglas FG, Chong PY. Influence of obesity on peripheral airways patency. Journal of applied physiology. 1972;33(5):559-63.

30. Adams JP, Murphy PG. Obesity in anaesthesia and intensive care. British journal of anaesthesia. 2000;85(1):91-108.

31. Chlif M, Keochkerian D, Choquet D, Vaidie A, Ahmaidi S. Effects of obesity on breathing pattern, ventilatory neural drive and mechanics. Respiratory physiology \& neurobiology. 2009;168(3):198202.

32. Chlif M, Keochkerian D, Feki Y, Vaidie A, Choquet D, Ahmaidi S. Inspiratory muscle activity during incremental exercise in obese men. International journal of obesity (2005). 2007;31(9):145663. 
33. Chlif M, Keochkerian D, Mourlhon C, Choquet D, Ahmaidi S. Noninvasive assessment of the tension-time index of inspiratory muscles at rest in obese male subjects. International journal of obesity (2005). 2005;29(12):1478-83.

34. Kress JP, Pohlman AS, Alverdy J, Hall JB. The impact of morbid obesity on oxygen cost of breathing (VO(2RESP)) at rest. Am J Respir Crit Care Med. 1999;160(3):883-6.

35. Kelly TM, Jensen RL, Elliott CG, Crapo RO. Maximum respiratory pressures in morbidly obese subjects. Respiration. 1988;54(2):73-7.

36. Sarikaya S, Cimen OB, Gokcay Y, Erdem R. Pulmonary function tests, respiratory muscle strength, and endurance of persons with obesity. Endocrinologist. 2003;13(2):136-41.

37. Magnani KL, Cataneo AJM. Respiratory muscle strength in obese individuals and influence of upper-body fat distribution. Sao Paulo Med J. 2007;125(4):215-9.

38. Weiner P, Waizman J, Weiner M, Rabner M, Magadle R, Zamir D. Influence of excessive weight loss after gastroplasty for morbid obesity on respiratory muscle performance. Thorax. 1998;53(1):39-42.

39. Sharp JT, Druz WS, Kondragunta VR. Diaphragmatic responses to body position changes in obese patients with obstructive sleep apnea. Am Rev Respir Dis. 1986;133(1):32-7.

40. Damsbo P, Vaag A, Hother-Nielsen O, Beck-Nielsen H. Reduced glycogen synthase activity in skeletal muscle from obese patients with and without type 2 (non-insulin-dependent) diabetes mellitus. Diabetologia. 1991;34(4):239-45.

41. Krotkiewski M, Grimby G, Holm G, Szczepanik J. Increased muscle dynamic endurance associated with weight reduction on a very-low-calorie diet. The American journal of clinical nutrition. 1990;51(3):321-30.

42. Salome CM, Munoz PA, Berend N, Thorpe CW, Schachter LM, King GG. Effect of obesity on breathlessness and airway responsiveness to methacholine in non-asthmatic subjects. Int J Obes (Lond). 2008;32(3):502-9. Epub 2007 Oct 23.

43. King GG, Brown NJ, Diba C, Thorpe CW, Munoz P, Marks GB, et al. The effects of body weight on airway calibre. Eur Respir J. 2005;25(5):896-901.

44. Malhotra A, Hillman D. Obesity and the lung: 3. Obesity, respiration and intensive care. Thorax. 2008;63(10):925-31. doi: 10.1136/thx.2007.086835.

45. Steier J, Lunt A, Hart N, Polkey MI, Moxham J. Observational study of the effect of obesity on lung volumes. Thorax. 2014;69(8):752-9. doi: 10.1136/thoraxjnl-2014-205148. Epub 2014 Apr 15.

46. Damia G, Mascheroni D, Croci M, Tarenzi L. Perioperative changes in functional residual capacity in morbidly obese patients. Br J Anaesth. 1988;60(5):574-8.

47. Littleton SW. Impact of obesity on respiratory function. Respirology. 2012;17(1):43-9. doi: 10.1111/j.440-843.2011.02096.x.

48. Hlavac MC, Catcheside PG, McDonald R, Eckert DJ, Windler S, McEvoy RD. Hypoxia impairs the arousal response to external resistive loading and airway occlusion during sleep. Sleep. 2006;29(5):624-31.

49. Yang AL, Lo MJ, Ting H, Chen JS, Huang CY, Lee SD. GABA(A) and GABA(B) receptors differentially modulate volume and frequency in ventilatory compensation in obese Zucker rats. Journal of applied physiology (Bethesda, Md : 1985). 2007;102(1):350-7. 
50. Lee SD, Nakano H, Farkas GA. Adenosinergic modulation of ventilation in obese zucker rats. Obesity research. 2005;13(3):545-55.

51. Nakano H, Lee SD, Farkas GA. Dopaminergic modulation of ventilation in obese Zucker rats. Journal of applied physiology (Bethesda, Md : 1985). 2002;92(1):25-32.

52. Norman RG, Goldring RM, Clain JM, Oppenheimer BW, Charney AN, Rapoport DM, et al. Transition from acute to chronic hypercapnia in patients with periodic breathing: predictions from a computer model. Journal of applied physiology (Bethesda, Md : 1985). 2006;100(5):173341.

53. Heinrich S, Birkholz T, Irouschek A, Ackermann A, Schmidt J. Incidences and predictors of difficult laryngoscopy in adult patients undergoing general anesthesia : a single-center analysis of 102,305 cases. Journal of anesthesia. 2013;27(6):815-21.

54. Shiga T, Wajima Z, Inoue T, Sakamoto A. Predicting difficult intubation in apparently normal patients: a meta-analysis of bedside screening test performance. Anesthesiology. 2005;103(2):42937.

55. Dority J, Hassan ZU, Chau D. Anesthetic implications of obesity in the surgical patient. Clinics in colon and rectal surgery. 2011;24(4):222-8.

56. Budde AO, Desciak M, Reddy V, Falcucci OA, Vaida SJ, Pott LM. The prediction of difficult intubation in obese patients using mirror indirect laryngoscopy: A prospective pilot study. Journal of anaesthesiology, clinical pharmacology. 2013;29(2):183-6.

57. Moore AR, Schricker T, Court O. Awake videolaryngoscopy-assisted tracheal intubation of the morbidly obese. Anaesthesia. 2012;67(3):232-5.

58. Lee BJ, Kang JM, Kim DO. Laryngeal exposure during laryngoscopy is better in the 25 degrees back-up position than in the supine position. British journal of anaesthesia. 2007;99(4):581-6.

59. Collins JS, Lemmens HJ, Brodsky JB, Brock-Utne JG, Levitan RM. Laryngoscopy and morbid obesity: a comparison of the "sniff" and "ramped" positions. Obesity surgery. 2004;14(9):1171-5.

60. Gupta D, Rusin K. Videolaryngoscopic endotracheal intubation (GlideScope) of morbidly obese patients in semi-erect position: a comparison with rapid sequence induction in supine position. Middle East journal of anaesthesiology. 2012;21(6):843-50.

61. Dixon BJ, Dixon JB, Carden JR, Burn AJ, Schachter LM, Playfair JM, et al. Preoxygenation is more effective in the 25 degrees head-up position than in the supine position in severely obese patients: a randomized controlled study. Anesthesiology. 2005;102(6):1110-5; discussion 5A.

62. Ramkumar V, Umesh G, Philip FA. Preoxygenation with 20 masculine head-up tilt provides longer duration of non-hypoxic apnea than conventional preoxygenation in non-obese healthy adults. Journal of anesthesia. 2011;25(2):189-94.

63. Ramachandran SK, Cosnowski A, Shanks A, Turner CR. Apneic oxygenation during prolonged laryngoscopy in obese patients: a randomized, controlled trial of nasal oxygen administration. Journal of clinical anesthesia. 2010;22(3):164-8.

64. Brismar B, Hedenstierna G, Lundquist H, Strandberg A, Svensson L, Tokics L. Pulmonary densities during anesthesia with muscular relaxation--a proposal of atelectasis. Anesthesiology. 1985;62(4):422-8. 
65. Gunnarsson L, Strandberg A, Brismar B, Tokics L, Lundquist H, Hedenstierna G. Atelectasis and gas exchange impairment during enflurane/nitrous oxide anaesthesia. Acta anaesthesiologica Scandinavica. 1989;33(8):629-37.

66. Pelosi P, Croci M, Ravagnan I, Tredici S, Pedoto A, Lissoni A, et al. The effects of body mass on lung volumes, respiratory mechanics, and gas exchange during general anesthesia. Anesthesia and analgesia. 1998;87(3):654-60.

67. Eichenberger A, Proietti S, Wicky S, Frascarolo P, Suter M, Spahn DR, et al. Morbid obesity and postoperative pulmonary atelectasis: an underestimated problem. Anesthesia and analgesia. 2002;95(6):1788-92, table of contents.

68. Reinius H, Jonsson L, Gustafsson S, Sundbom M, Duvernoy O, Pelosi P, et al. Prevention of atelectasis in morbidly obese patients during general anesthesia and paralysis: a computerized tomography study. Anesthesiology. 2009;111(5):979-87.

69. Aldenkortt M, Lysakowski C, Elia N, Brochard L, Tramer MR. Ventilation strategies in obese patients undergoing surgery: a quantitative systematic review and meta-analysis. British journal of anaesthesia. 2012;109(4):493-502.

70. Yu G, Yang K, Baker AB, Young I. The effect of bi-level positive airway pressure mechanical ventilation on gas exchange during general anaesthesia. British journal of anaesthesia. 2006;96(4):522-32.

71. Ebeo CT, Benotti PN, Byrd RP, Jr., Elmaghraby Z, Lui J. The effect of bi-level positive airway pressure on postoperative pulmonary function following gastric surgery for obesity. Respir Med. 2002;96(9):672-6.

72. Joris JL, Sottiaux TM, Chiche JD, Desaive CJ, Lamy ML. Effect of bi-level positive airway pressure (BiPAP) nasal ventilation on the postoperative pulmonary restrictive syndrome in obese patients undergoing gastroplasty. Chest. 1997;111(3):665-70.

73. Ferreyra G, Long Y, Ranieri VM. Respiratory complications after major surgery. Curr Opin Crit Care. 2009;15(4):342-8. doi: 10.1097/MCC.0b013e32832e0669.

74. Jaber S, Chanques G, Jung B. Postoperative noninvasive ventilation. Anesthesiology. 2010;112(2):453-61. doi: 10.1097/ALN.0b013e3181c5e5f2.

75. Ferreyra GP, Baussano I, Squadrone V, Richiardi L, Marchiaro G, Del Sorbo L, et al. Continuous positive airway pressure for treatment of respiratory complications after abdominal surgery: a systematic review and meta-analysis. Ann Surg. 2008;247(4):617-26. doi: 10.1097/ SLA.0b013e3181675829.

76. Ireland CJ, Chapman TM, Mathew SF, Herbison GP, Zacharias M. Continuous positive airway pressure (CPAP) during the postoperative period for prevention of postoperative morbidity and mortality following major abdominal surgery. Cochrane Database Syst Rev. 2014;8:CD008930. (doi):10.1002/14651858.CD008930.pub2.

77. van Kaam AH, Lachmann RA, Herting E, De Jaegere A, van Iwaarden F, Noorduyn LA, et al. Reducing atelectasis attenuates bacterial growth and translocation in experimental pneumonia. Am J Respir Crit Care Med. 2004;169(9):1046-53. Epub 2004 Feb 20. 
78. Carron M, Zarantonello F, Tellaroli P, Ori C. Perioperative noninvasive ventilation in obese patients: a qualitative review and meta-analysis. Surgery for obesity and related diseases : official journal of the American Society for Bariatric Surgery. 2015:013.

79. Bachler T, Schiesser M, Lutz TA, le Roux CW, Bueter M. Where to begin and where to end? Preoperative assessment for patients undergoing metabolic surgery. Dig Surg. 2014;31(1):25-32. doi: 10.1159/000354553. Epub 2014 May 8.

80. Purnell JQ, Selzer F, Smith MD, Berk PD, Courcoulas AP, Inabnet WB, et al. Metabolic syndrome prevalence and associations in a bariatric surgery cohort from the Longitudinal Assessment of Bariatric Surgery-2 study. Metab Syndr Relat Disord. 2014;12(2):86-94. doi: 10.1089/ met.2013.0116. Epub 2013 Dec 31.

81. Sheu EG, Channick R, Gee DW. Improvement in severe pulmonary hypertension in obese patients after laparoscopic gastric bypass or sleeve gastrectomy. Surg Endosc. 2016;30(2):633-7. doi: 10.1007/s00464-015-4251-5. Epub 2015 Jun 20.

82. van Huisstede A, Biter LU, Luitwieler R, Castro Cabezas M, Mannaerts G, Birnie E, et al. Pulmonary function testing and complications of laparoscopic bariatric surgery. Obesity surgery. 2013;23(10):1596-603.

83. Sood A, Abdollah F, Sammon JD, Majumder K, Schmid M, Peabody JO, et al. The Effect of Body Mass Index on Perioperative Outcomes After Major Surgery: Results from the National Surgical Quality Improvement Program (ACS-NSQIP) 2005-2011. World J Surg. 2015;39(10):2376-85. doi: 10.1007/s00268-015-3112-7.

84. Chung F, Abdullah HR, Liao P. STOP-Bang Questionnaire: A practical approach to screen for obstructive sleep apnea. Chest. 2015.

85. Chung F, Yang Y, Liao P. Predictive performance of the STOP-Bang score for identifying obstructive sleep apnea in obese patients. Obesity surgery. 2013;23(12):2050-7.

86. Chung F, Yegneswaran B, Liao P, Chung SA, Vairavanathan S, Islam S, et al. STOP questionnaire: a tool to screen patients for obstructive sleep apnea. Anesthesiology. 2008;108(5):812-21.

87. Proczko MA, Stepaniak PS, de Quelerij M, van der Lely FH, Smulders JF, Kaska L, et al. STOPBang and the effect on patient outcome and length of hospital stay when patients are not using continuous positive airway pressure. Journal of anesthesia. 2014;28(6):891-7.

88. Hamoui N, Anthone G, Crookes PF. The value of pulmonary function testing prior to bariatric surgery. Obesity surgery. 2006;16(12):1570-3.

89. Nepomnayshy D, Hesham W, Erickson B, MacDonald J, Iorio R, Brams D. Sleep apnea: is routine preoperative screening necessary? Obesity surgery. 2013;23(3):287-91.

90. Peromaa-Haavisto P, Tuomilehto H, Kossi J, Virtanen J, Luostarinen M, Pihlajamaki J, et al. Prevalence of Obstructive Sleep Apnoea Among Patients Admitted for Bariatric Surgery. A Prospective Multicentre Trial. Obesity surgery. 2015.

91. Barbalho-Moulim MC, Miguel GP, Forti EM, Campos Fdo A, Costa D. Effects of preoperative inspiratory muscle training in obese women undergoing open bariatric surgery: respiratory muscle strength, lung volumes, and diaphragmatic excursion. Clinics (Sao Paulo, Brazil). 2011;66(10):1721-7. 
92. van Huisstede A, Rudolphus A, Castro Cabezas M, Biter LU, van de Geijn GJ, Taube C, et al. Effect of bariatric surgery on asthma control, lung function and bronchial and systemic inflammation in morbidly obese subjects with asthma. Thorax. 2015;70(7):659-67.

93. Thomas PS, Cowen ER, Hulands G, Milledge JS. Respiratory function in the morbidly obese before and after weight loss. Thorax. 1989;44(5):382-6. 


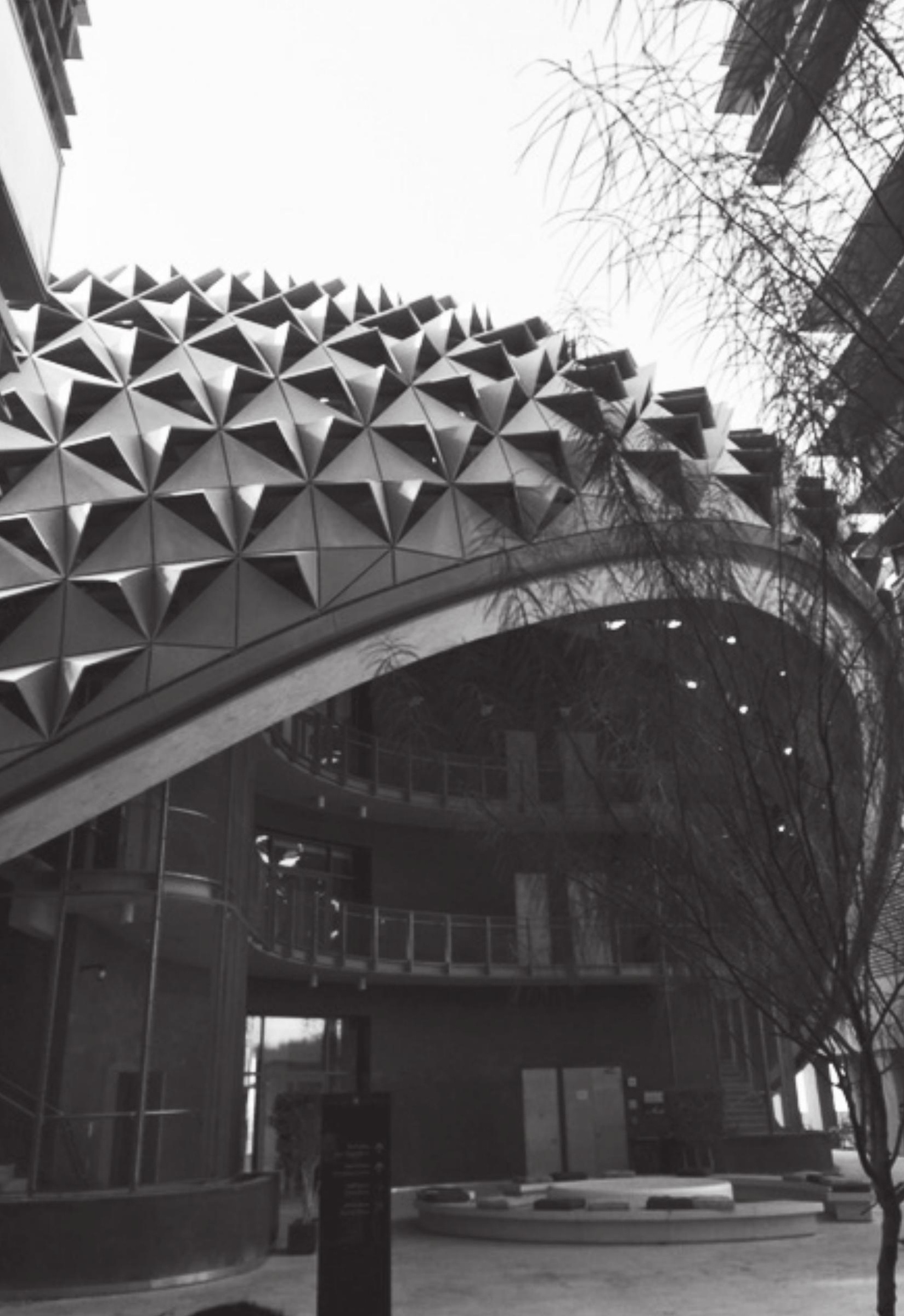




\title{
6. EFFECTS OF BARIATRIC SURGERY ON INSPIRATORY MUSCLE STRENGTH
}

\author{
Sjaak Pouwels ${ }^{1,2}$ \\ Marieke Kools-Aarts ${ }^{1}$ \\ Mohammed Said ${ }^{1}$ \\ Joep A.W. Teijink ${ }^{1,2}$ \\ Frank W.J.M. Smeenk ${ }^{3}$ \\ Simon W. Nienhuijs ${ }^{1}$
}

Springerplus. 2015 Jul 7;4:322-329.

1. Department of Surgery, Catharina Hospital, Eindhoven, The Netherlands

2. Department of Epidemiology, CAPHRI Research School, Maastricht University, The Netherlands

3. Department of Respiratory Medicine, Catharina Hospital, Eindhoven, The Netherlands 


\section{ABSTRACT}

\section{Background}

The respiratory function is affected by obesity due to an increased deposition of fat on the chest wall. The objective of this study was to investigate the strength of the inspiratory respiratory muscles of obese individuals and the possible influence of bariatric surgery on it by measuring the Maximum Inspiratory Pressure.

\section{Methods}

Patients referred to a bariatric centre between the $3^{\text {rd }}$ of October 2011 and the $3^{\text {rd }}$ of May 2012 were screened preoperatively by a multidisciplinary team. Their Maximum Inspiratory Pressure (MIP) was measured at screening and 3, 6 and 9 months postoperative. In case of a preoperative MIP lower than $70 \%$ of predicted pressure training was provided supervised by a physiotherapist.

\section{Results}

The mean age of 124 included patients was $42.9 \pm 11.0$ years and mean BMI was $43.1 \pm 5.2 \mathrm{~kg} /$ $\mathrm{m}^{2}$. The mean predicted MIP preoperatively was $127 \pm 31$ in $\mathrm{cm}_{2} \mathrm{O}$ and the mean measured MIP was $102 \pm 24$ in $\mathrm{cm} \mathrm{H}_{2} \mathrm{O}$. Three patients (2.4\%) received training. Three months after surgery the MIP was $76 \pm 26 \mathrm{~cm} \mathrm{H}_{2} \mathrm{O}$, after 6 months $82 \pm 28 \mathrm{~cm} \mathrm{H}_{2} \mathrm{O}$ and after 9 months 86 $\pm 28 \mathrm{~cm} \mathrm{H}_{2} \mathrm{O}$. All postoperative measurements were significant lower than preoperatively $(\mathrm{P}<0.05)$. The only influencing factor for the preoperative MIP was age $(\mathrm{p}=0.014)$.

\section{Conclusion}

The preoperative MIP values were significantly lower than the predicted MIP values, probably due to altered respiratory mechanics. 


\subsection{INTRODUCTION}

Obesity is a chronic disease characterised by an excessive accumulation of body fat and causes damage to various body functions, such as cardiovascular, musculoskeletal, and metabolic functions amongst others. (1) Also the respiratory function is affected by obesity due to an increased deposition of fat on the chest wall. This causes a reduction in thoracic compliance, lung volumes and capacities. (2-4) Respiratory dysfunction in this patient population can occur due to changes in the relationship between lung, chest wall and diaphragm, causing an impairment of respiratory mechanics and also changes in gas exchange. (5)

Several studies have demonstrated that weight loss due to bariatric surgery has resulted in a huge improvement in some functions, such as a decrease in haemoglobin and haematocrit (6), decreased heart rate and oxygen consumption (6) and a reduction in insulin resistance. Bariatric surgery also showed an improved lung function with increased forced vital capacity (FVC) $(7,8)$, forced expiratory volume in one second (FEV1), and improved alveolar-capillary diffusion capacity (6) and an improvement in gas exchange $(7,8)$. However, studies on the respiratory muscle function of the obese population (before and after bariatric surgery) show conflicting results. $(5,9-12)$

Thus, the objective of this study was to investigate the strength of the respiratory muscles of obese individuals (before and after bariatric surgery) by measuring the maximum inspiratory pressure (MIP). Also we have investigated the influence of patient related factors and type of operation (sleeve gastrectomy or gastric bypass) on the MIP before and after bariatric surgery. Furthermore, the association of the body mass index (BMI) and the presence of comorbidities with the respiratory muscle strength were reviewed.

\subsection{METHODS}

This study was approved by the Medical Ethics Committee of the Catharina Hospital Eindhoven and adheres the principles of the declaration of Helsinki. Informed consent was obtained of all patients included.

\section{PATIENT POPULATION}

Patients were referred to a bariatric centre by their general practitioner or other physicians. A multidisciplinary team screened all patients preoperatively, consisting of a physician assistant, a psychologist, a nutritionist and a surgeon. Patients were eligible for surgery if they had a BMI of $40 \mathrm{~kg} / \mathrm{m}^{2}$ or higher, or a BMI between 35 and $40 \mathrm{~kg} / \mathrm{m}^{2}$ with significant co-morbidities, 
with serious attempts to lose weight in the past. Co-morbidities were considered significant when medication had to be used, or if continuous positive airway pressure (CPAP) had to be used in case of obstructive sleep apnoea (OSAS). The indication for a bariatric procedure was made in a multidisciplinary consultation, taking into account the patients' preference, age, polypharmacy, reflux complaints, body composition in relation to BMI and bowel diseases. Patients with pre-existing pulmonary and/or neuromuscular diseases were excluded from this study.

\section{RESPIRATORY MUSCLE FUNCTION AND MIP ASSESSMENT}

Respiratory muscle strength was assessed by measuring the maximal inspiratory pressure (MIP) with a digital mouth pressure meter $\left( \pm 300 \mathrm{~cm} \mathrm{H}_{2} \mathrm{O}\right)$. The manometer (Micro RPM ${ }^{\circledast}$, Carefusion, USA) was calibrated in accordance with the manufacturer's recommendations.

The maximal static respiratory pressure generated in the mouth, after complete inhalation and exhalation, carries out the measurement of respiratory muscle strength, divided in the Maximal Inspiratory Pressure (MIP) and Maximal Expiratory Pressure (MEP), which are indicative of the strength of the inspiratory and expiratory muscle groups. (14) MIP is a measure of inspiratory muscle strength (which is representative for the function of the diaphragm), whereas MEP measures the strength of the abdominal and intercostal muscles. $(15-17)$

The assessment of the respiratory muscle strength took place before bariatric surgery and 3, 6 and 9 months postoperatively by an independent and trained physiotherapist using the ATS/ ERS guidelines. $(13,18)$ The MIP values were compared to each other and to the normal values adjusted for their sex and age. (19)

When the actual preoperative measured MIP was $\leq 70 \%$ of the predicted MIP, patients were asked to conduct respiratory function training prior to surgery. The protocol for training of the respiratory function was adapted from a study of Hulzebos and colleagues. (20)

\section{DATA COLLECTION}

Data were collected prospectively using an online registry (Patients Outcome Measurement Tool). The patients who got approval between the $3^{\text {rd }}$ of October 2011 and the $3^{\text {rd }}$ of May 2012 were included in this study as during this period the inspiratory muscle function was measured.

Data of interest were MIP values, patients' characteristics, operative details, hospital stay and evolution on weight and comorbidities. Furthermore were included the postoperative 
pulmonary complications defined as pulmonary infections as determined by the physician, thromboembolic events, and respiratory distress resulting into additional care.

\section{STATISTICAL ANALYSIS}

Continuous variables were presented as mean \pm standard deviation (SD). Categorical variables were presented as frequency with percentages. The Shapiro-Wilk test was used to test each variable for normality. Student's t-test for independent groups or the Mann-Whitney U test was used to compare the MIP values in the preoperative period and changes in MIP values over time, depending on the normality or non-normality of the data distribution. To compare the MIP values at different times (preoperatively, and after 3, 6 and 9 months after surgery), repeated measures ANOVA was used. To assess postoperative MIP values (and the difference with preoperative MIP values) between patients who had a sleeve gastrectomy and gastric bypass, the two-repeated ANOVA was used.

The Pearson correlation coefficient was used to determine the correlation between de following parameters: preoperative BMI and preoperative MIP; Prevalence of OSAS and preoperative MIP; weight loss 3 months after surgery and MIP 3 months after surgery; weight loss 9 months after surgery and MIP 9 months after surgery; smoking and MIP values (preoperative and after 3, 6 and 9 months); gender and preoperative MIP and age and preoperative MIP.

In all tests, values of $\mathrm{p}<0.05$ were considered statistically significant. Statistical Package for Social Sciences (SPSS, Chicago, IL, USA Version 20.0) was used to prepare the database and for statistical analysis.

\subsection{RESULTS}

\section{PATIENTS AND PROCEDURES}

All 124 patients completed the 9 months follow-up and were all available for the final analysis. The majority of the included 124 patients were female $(n=106)$. The mean age was $42.9 \pm 11.0$ years and mean BMI was $43.1 \pm 5.2 \mathrm{~kg} / \mathrm{m}^{2}$. Related comorbidities encountered were diabetes (20.2\%), OSAS (13.7\%), hypertension (33.9\%) and dyslipidaemia (20.2\%). Among the patients 17 (13.7\%) were tobacco users and 11 (8.9\%) used alcohol. Half the patients underwent a sleeve gastrectomy $(n=60 ; 49.2 \%)$ and the other half received a gastric bypass $(n=64 ; 50.8 \%)$. These interventions resulted into a weight loss of $18 \pm 26 \mathrm{~kg}(13 \pm 21 \% \mathrm{TWL})$ and $51 \pm 29$ $\mathrm{kg}(41 \pm 23 \% \mathrm{TWL})$ respectively at three months and one year postoperative. No pulmonary complications as defined in the methods section were seen postoperatively. 


\section{MAXIMAL INSPIRATORY PRESSURES}

In the preoperative period, the mean predicted MIP was $127 \pm 31$ in $\mathrm{cm} \mathrm{H}_{2} \mathrm{O}$ and the mean measured MIP was $102 \pm 24$ in $\mathrm{cm} \mathrm{H}_{2} \mathrm{O}$ (Table 6.1). Based on these results, only three patients were asked to train their respiratory function, because they had a preoperative measured MIP value $<70 \%$ of predicted. Three months after surgery the MIP was $76 \pm 26 \mathrm{~cm} \mathrm{H}_{2} \mathrm{O}$, after 6 months the MIP was $82 \pm 28 \mathrm{~cm} \mathrm{H}_{2} \mathrm{O}$ and after 9 months $86 \pm 28 \mathrm{~cm} \mathrm{H}_{2} \mathrm{O}$. At all three measure moments this decrease was significant compared to the preoperative value $(\mathrm{P}=0.01)$.

TABLE 6.1. Maximal inspiratory pressures of obese patients before and after a bariatric procedure

\begin{tabular}{|c|c|c|c|c|}
\hline $\begin{array}{c}\text { Predicted MIP* } \\
(\mathrm{n}=124)\end{array}$ & $\begin{array}{c}\text { Actual MIP preoperative* } \\
(\mathrm{n}=124)\end{array}$ & $\begin{array}{c}\text { After } 3 \text { months* } \\
(\mathrm{n}=124)\end{array}$ & $\begin{array}{c}\text { After } 6 \text { months* } \\
(\mathrm{n}=124)\end{array}$ & $\begin{array}{c}\text { After } 9 \text { months* }^{*} \\
(\mathrm{n}=124)\end{array}$ \\
\hline $127 \pm 31$ & $102 \pm 24^{\mathrm{A}}$ & $76 \pm 26^{\mathrm{B}, \mathrm{C}}$ & $82 \pm 28^{\mathrm{B}, \mathrm{C}}$ & $86 \pm 28^{\mathrm{B}, \mathrm{C}}$ \\
\hline
\end{tabular}

* In $\mathrm{cm} \mathrm{H}_{2} \mathrm{O}$

${ }^{A}$ significant difference compared with Predicted MIP $(p=0.019)$

${ }^{B}$ significant difference compared with Actual MIP $(\mathrm{p}=0.01)$

${ }^{\mathrm{C}}$ no significant difference between 3,6 and 9 month values $(\mathrm{p}=0.273$ )

TABLE 6.2. Mean MIP changes ( \pm SD) in $\mathrm{cm}_{2} \mathrm{O}$ between different time points

\begin{tabular}{|l|c|c|c|}
\hline \multicolumn{2}{|c|}{ MIP after 3 months } & MIP after 6 mont hs & MIP after 9 months \\
\hline Compared with: & $-26.65 \pm 22.30$ & $-20.61 \pm 20.58$ & $-16.54 \pm 21.08$ \\
\hline Actual Preop MIP & & $6.03 \pm 19.99$ & $10.11 \pm 20.65$ \\
\hline MIP after 3 months & & & $4.07 \pm 11.98$ \\
\hline MIP after 6 months & & & \\
\cline { 1 - 1 } MIP after 9 months & & & \\
\hline
\end{tabular}

\section{SLEEVE GASTRECTOMY VERSUS GASTRIC BYPASS}

The preoperative MIP, the MIP 3 months after surgery, 6 months after surgery and 9 months after surgery did not significantly differ between patients who had a sleeve gastrectomy compared to a gastric bypass $(\mathrm{P}=0.590, \mathrm{P}=0.592, \mathrm{P}=0.165$ and $\mathrm{P}=0.895$ respectively).

\section{CHANGES IN MIP VALUES OVER TIME}

Table 6.2 gives an overview of the mean MIP changes between different time points. There was a significant difference between the mean changes in MIP after 3 months and 6 months (respectively $-26.65 \pm 22.30$ and $6.03 \pm 19.99 \mathrm{~cm} \mathrm{H}_{2} \mathrm{O}, \mathrm{p}=0.001$ ) and there was a significant difference in the mean changes in MIP after 3 and after 9 months (respectively $-26.65 \pm 22.30$ and $\left.4.07 \pm 11.98 \mathrm{~cm} \mathrm{H}_{2} \mathrm{O}, \mathrm{p}=0.001\right)$ 


\section{CORRELATIONS BETWEEN DIFFERENT VARIABLES}

Table 6.3 gives an overview of the correlations investigated. There was no linear correlation between the preoperative BMI and preoperative MIP $(r=-0.045, p=0.619)$, the prevalence of OSAS and the preoperative MIP $(r=-0.025, p=0.779)$, weight loss 3 months after surgery and MIP 3 months after surgery ( $\mathrm{r}=-0.187, \mathrm{p}=0.008)$, weight loss 9 months after surgery and MIP 9 months after surgery $(\mathrm{r}=-0.075, \mathrm{p}=0.072)$ and smoking and the MIP values preoperative $(\mathrm{r}=-0.003, \mathrm{p}=0.976)$ and 3,6 and 9 months postoperative $(\mathrm{r}=0.070, \mathrm{p}=0.439 ; \mathrm{r}=0.054, \mathrm{p}=0.552$; and $\mathrm{r}=0.014, \mathrm{p}=0.877$ respectively). The correlation between gender and the preoperative MIP showed a trend towards significance $(\mathrm{r}=-0.155, \mathrm{p}=0.085)$. The only (negative) linear correlation found was between age and the preoperative MIP $(r=-0.220, p=0.014)$.

TABLE 6.3. Overview of the correlations between different variables ( $n=124$ patients)

\begin{tabular}{|l|l|c|c|}
\hline Variable 1 & Variable 2 & $\begin{array}{c}\text { Correlation } \\
\text { coefficient }\end{array}$ & P-value \\
\hline Preoperative BMI & Preoperative MIP & $\mathrm{r}=-0.045$ & $\mathrm{p}=0.619$ \\
\hline Prevalence of OSAS & Preoperative MIP & $\mathrm{r}=-0.025$ & $\mathrm{p}=0.779$ \\
\hline Weight loss 3 months after surgery & MIP 3 months after surgery & $\mathrm{r}=-0.075$ & $\mathrm{p}=0.408$ \\
\hline Weight loss 9 months after surgery & MIP 9 months after surgery & $\mathrm{r}=-0.187$ & $\mathrm{p}=0.072$ \\
\hline & MIP preoperative & $\mathrm{r}=-0.003$ & $\mathrm{p}=0.976$ \\
& MIP after 3 months & $\mathrm{r}=0.070$ & $\mathrm{p}=0.439$ \\
& MIP after 6 months & $\mathrm{r}=0.054$ & $\mathrm{p}=0.552$ \\
& MIP after 9 months & $\mathrm{r}=0.014$ & $\mathrm{p}=0.877$ \\
\hline Gender & Preoperative MIP & $\mathrm{r}=-0.155$ & $\mathrm{p}=0.085$ \\
\hline Age & Preoperative MIP & $\mathbf{r}=-\mathbf{0 . 2 2 0}$ & $\mathrm{p}=0.014$ \\
\hline
\end{tabular}

\subsection{DISCUSSION}

The actual measured MIP was significantly lower than the predicted values in the present study. The Maximum Inspiratory Pressures at 3, 6 and 9 months after bariatric surgery were decreased significantly compared with the preoperative MIP. The only (negative) linear correlation found was between the age and the preoperative MIP, which is corresponding with earlier studies on this subject. $(5,19)$

Obesity has a detrimental effect on the pulmonary physiology, including respiratory mechanics, airway resistance, respiratory muscle function, lung volume, work of breathing (WOB) and gas exchange. (21) Morbidly obese patients present with increased metabolic demands due to a deposition of fat in the chest wall, which results in an increased mass to move during breaths and therefore a higher WOB. This elevated WOB results in reduced 
chest wall compliance. Also there is an elevation of the diaphragm, which (upon contracting) acts under pressure of a distended abdomen. (22-24)

This 'overload' triggers a variety of mechanisms in the activity of the respiratory muscles and causes a long term training effect, which can increase muscle strength. $(5,22,23)$ It is believed that this muscle strength decreases when patients develop a condition such as OSAS. (5) In the postoperative period, weight reduction may promote an improvement in respiratory mechanics and compliance, improving the efficiency of the respiratory muscles. (4) In the present study, the MIP after was decreased 3 months after surgery, but at 6 months and 9 months after surgery it increased. This is the effect of weight loss, which reduces the earlier mentioned 'overload' and therefore creates a new setpoint to which the respiratory muscle strength has to adjust. This could be an explanation for the fact that the MIP decreased 3 months after surgery in our study. However we found a trend towards a negative correlation between weight loss 9 months after surgery and the MIP 9 months after surgery $(p=0.072)$, which indicates a decrease in respiratory muscle strength when the weight loss increases. The explanation for this matter lies in the earlier mentioned 'overload', which vanishes after successful bariatric surgery. Whether this earlier mentioned new 'setpoint is due to an improvement in diaphragm muscle function or a change lung compliance is unknown.

Also animal studies in rabbits show that chronically increased intra-abdominal pressure induce several histological and cellular changes in composition of greater abdominal muscles, especially the rectus abdominis and diaphragm muscle. (25-28) Changes in muscle fiber composition of the muscles were observed, an increased ratio of type II muscle fibers that are mainly anaerobically active. (25-28)

Studies investigating indices of respiratory muscle strength in obese patients and comparing them with eutrophic individuals or comparing them with normal values showed no consistent results. $(3,5,10)$ Kelly et al. (10) found no significant results in MIP values among obese individuals (with an average of $183 \%$ of the predicted weight) and individuals with an average of $99 \%$ of the weight. Sarikaya et al. (3) showed a significantly reduced MIP in obese individuals with no significant difference compared with eutrophic individuals. Magnani et al. (5) found that the MIP was within normal values for age and gender in 99 obese individuals (23 men and 76 women). There was no significant difference between different BMI groups $\left(35-40,40-45,45-50\right.$ and $\left.\geq 50 \mathrm{~kg} / \mathrm{m}^{2}\right)$. (5)

In the literature investigating the effect of bariatric surgery on respiratory pressures, there are also conflicting results. Weiner et al. (4) showed that maximum respiratory pressures increased 6 months after bariatric surgery when compared with the preoperative values in 21 obese patients. 
Parreira et al. (23) assessed the MIP of 30 morbidly obese patients (24 women and 6 men) preoperatively and 1 and 6 months after surgery. They found no significant difference in MIP 1 and 6 months after surgery compared to the preoperative values (preoperative MIP: $96 \pm 35$; after 1 month: $100 \pm 38$; after 6 months: $104 \pm 33 \mathrm{~cm} \mathrm{H}_{2} \mathrm{O}$ ). (23) When comparing the MIP values of 17 individuals 36 months after surgery, they found a significantly increased MIP $\left(121 \pm 35 \mathrm{~cm} \mathrm{H}_{2} \mathrm{O}\right)$ compared to the preoperative MIP $\left(96 \pm 35 \mathrm{~cm} \mathrm{H}_{2} \mathrm{O}\right)$. (23)

Cherniack and colleagues (29) found that obese individuals have inefficient respiratory muscles due to reduced chest wall compliance or lower lung volume. Also the MIP was lower than the predicted value. (29) Wadström and coworkers (30) found that, despite a weight loss of $18 \%$ and improvement of lung volumes, the included obese individuals showed no significant change in respiratory muscle strength.

Barbalho-Moulim et al. (15) randomised 32 obese women undergoing elective open bariatric surgery to either an inspiratory muscle-training group or usual care group. Compared to the preoperative values, the MIP decreased significantly in both groups. However the reduction in MIP was $28 \%$ in the inspiratory muscle training group en $47 \%$ in the usual care group. (15) In a different patient cohort (24 obese women scheduled for Roux en-Y gastric bypass), Barbalho-Moulim et al. (16) found that compared to preoperative MIP values, the MIP 1 year after bariatric surgery significantly decreased (preoperative: $78.75 \pm 20.07 \mathrm{~cm} \mathrm{H}_{2} \mathrm{O}$; 1 year after surgery: $69.17 \pm 18.86 \mathrm{~cm} \mathrm{H}_{2} \mathrm{O}, \mathrm{p}=0.0183$ ). (16)

Various studies have examined the correlation between MIP and body composition but found different results. Vincken and colleagues (31) found that body composition did not contribute in explaining MIP variability. Enright et al. (32) reported that weight and waist circumference was negatively related to the MIP. Carpenter et al. (33) showed that individuals with a higher BMI had a lower MIP. We have not found a significant correlation between body composition and MIP. A possible explanation could be the difference in sample size, than the earlier mentioned studies. (31-33)

Different results were mentioned about the correlation between smoking status and MIP. Hautmann et al. (34) demonstrated that there was no significant relation between smoking status and MIP. Leech et al. (35) also did not found a significant relation between smoking status and MIP. Therefore Enright et al. (36) found that smokers had a 15\% lower MIP than non-/former smokers. In our study we could not find a relationship between smoking and the MIP values. A possible explanation for this matter is the small number of smokers in our study population $(17(13.7 \%))$. 
Our study has several limitations. First, our male/female equilibrium was not equal. The majority of our patients were female. Second, there is a known difference between the MIP values between males and females $(33,34)$, which could be a confounding factor in the interpretation of the results.

The significant difference between the predictive MIP and the preoperative measured MIP was found using the equation by Wilson et al. (19) This could imply two remarks. First, is unknown whether or not the used predictive equation is suitable for the obese population and secondly other commonly used predictive equations could influence the results. $(12,14$, $37,38)$

The clinical relevancy of measuring the MIP prior to and after bariatric surgery is questionable. Two questions need to be answered to determine the clinical relevancy 1) How many bariatric surgical teams measure MIP routinely and 2) if the MIP is measured, what is its influence on the decision-making progress in qualification and preparation of patients for bariatric surgery?

The earlier mentioned study of Barbalho-Moulim et al. (15) found that inspiratory muscle training prevented a reduction in MIP postoperatively. Another study by Barbalho-Moulim (39) compared the effect of laparoscopic bariatric surgery with open bariatric surgery on the lung function, without preoperative training of the patients. In both groups, there was a decrease in MIP postoperatively, $23 \%$ in the laparoscopic group compared to $37 \%$ in the open group. (39) Both studies did not investigate the effect on postoperative (pulmonary) complications.

In the current Dutch bariatric practice, almost all bariatric interventions are performed via laparoscopic procedures and pulmonary complications are rarely seen. Also in this study no pulmonary complications were seen. Therefore in other types of surgery, per example cardio-thoracic surgery, determining the MIP is useful for clinical purposes. This because after coronary artery bypass grafting surgery, pulmonary complications are more frequently seen and therefore inspiratory muscle training. (20) In our opinion, based on the current body of literature, the clinical relevance of measuring MIP prior to bariatric surgery remains questionable. Therefore further research is needed to investigate the clinical usefulness of this MIP measurement, especially in patients with obesity-induced respiratory dysfunction. 


\subsection{CONCLUSION}

The preoperative Maximum Inspiratory Pressure values were significantly lower than the predicted MIP values. Only three patients were indicated to train their respiratory function preoperatively. Also a significant decrease in maximum pressures was found 3, 6 and 9 months after bariatric surgery each compared to the preoperative measurements. A negative significant correlation was observed between age and preoperative MIP. Due to conflicting results in the current literature, the low number of pulmonary complications seen after bariatric surgery the clinical relevancy of measuring the MIP prior to and after bariatric surgery remains questionable. 


\subsection{REFERENCES}

1. WHO, "Obesity: preventing and Managing the global epidemic," Report of a WHO Consultation. 2000;894(1-12):1-253.

2. Hamoui N, Anthone G, Crookes PF. The value of pulmonary function testing prior to bariatric surgery. Obesity Surgery. 2006;16(12):1570-3.

3. Sarikaya S, Cimen OB, Gokcay Y, Erdem R. Pulmonary function tests, respiratory muscle strength, and endurance of persons with obesity. Endocrinologist. 2003;13(2):136-41.

4. Weiner P, Waizman J, Weiner M, Rabner M, Magadle R, Zamir D. Influence of excessive weight loss after gastroplasty for morbid obesity on respiratory muscle performance. Thorax. 1998;53(1):39-42.

5. Magnani KL, Cataneo AJM. Respiratory muscle strength in obese individuals and influence of upper-body fat distribution. Sao Paulo Med J. 2007;125(4):215-9.

6. Zavorsky GS, Kim DJ, Sylvestre JL, Christou NV. Alveolar-membrane diffusing capacity improves in the morbidly obese after bariatric surgery. Obesity Surgery. 2008;18(2):256-63.

7. Zavorsky GS, Murias JS, Kim DJ, Gow J, Sylvestre JL, Christou NV. Waist-to-hip ratio is associated with pulmonary gas exhange in the morbidly obese. Chest. 2007;131(2):362-7.

8. Davila-Cervantes A, Domınguez-Cherit G, Borunda D. Impact of surgically-induced weight loss on respiratory function: a prospective analysis Obesity Surgery. 2004;14(10):1389-92.

9. Wadstrom C, Muller-Suur R, Backman L. Influence of excessive weight loss on respiratory function. A study of obese patients following gastroplasty. Eur J Surg. 1991;157(5):341-6.

10. Kelly TM, Jensen RL, Elliott CG, Crapo RO. Maximum respiratory pressures in morbidly obese subjects. Respiration. 1988;54(2):73-7.

11. Sampson MG, Grassino AE. Load compensation in obese patients during quiet tidal breathing. J Appl Physiol. 1983;55(4):1269-76.

12. Black LF, Hyatt RE. Maximal respiratory pressures: normal values and relationship to age and sex. Am Rev Respir Dis. 1969;99:696-702.

13. Celli BR, MacNee W. Standards for the diagnosis and treatment of patients with COPD: a summary of the ATS/ERS position paper. The European respiratory journal. 2004;23(6):932-46.

14. Costa D, Goncalves HA, Lima LP, Ike D, Cancelliero KM, Montebelo MI. New reference values for maximal respiratory pressures in the Brazilian population. Jornal brasileiro de pneumologia : publicacao oficial da Sociedade Brasileira de Pneumologia e Tisilogia. 2010;36(3):306-12.

15. Barbalho-Moulim MC, Miguel GP, Forti EM, Campos Fdo A, Costa D. Effects of preoperative inspiratory muscle training in obese women undergoing open bariatric surgery: respiratory muscle strength, lung volumes, and diaphragmatic excursion. Clinics (Sao Paulo, Brazil). 2011;66(10):1721-7.

16. Barbalho-Moulim MC, Miguel GP, Forti EM, Campos Fdo A, Peixoto-Souza FS, Costa D. Pulmonary Function after Weight Loss in Obese Women Undergoing Roux-en-Y Gastric Bypass: One-Year Followup. ISRN obesity. 2013;2013:796454. 
17. Pazzianotto-Forti EM, Peixoto-Souza FS, Piconi-Mendes C, Rasera-Junior I, Barbalho-Moulim M. Behavior of respiratory muscle strength in morbidly obese women by using different predictive equations. Rev Bras Fisioter. 2012;16(6):479-86.

18. Standards for the diagnosis and care of patients with chronic obstructive pulmonary disease. American Thoracic Society. Am J Respir Crit Care Med. 1995;152(5 Pt 2):S77-121.

19. Wilson SH, Cooke NT, R.H.T. E, Spiro SG. Predicted normal values for maximal respiratory pressures in caucasian adults and children. Thorax. 1984;39:535-8.

20. Hulzebos EH, Helders PJ, Favie NJ, De Bie RA, Brutel de la Riviere A, Van Meeteren NL. Preoperative intensive inspiratory muscle training to prevent postoperative pulmonary complications in high-risk patients undergoing CABG surgery: a randomized clinical trial. Jama. 2006;296(15):1851-7.

21. Koenig SM. Pulmonary complications of obesity. American Journal of the Medical Sciences. 2001;321(4):249-79.

22. Wei YF, Wu HD. Candidates for Bariatric Surgery: Morbidly Obese Patients with Pulmonary Dysfunction. Journal Of Obesity. 2012.

23. Parreira VF, Matos CMP, Athayde FTS, Moraes KS, Barbosa KS, Britto RR. Evolution of respiratory muscle strength in post-operative gastroplasty. Rev Bras Fisioter. 2012;16(3):225-30.

24. Jubber AS. Respiratory complications of obesity. Journal of Clinical Practice. 2004;58(6):573-80.

25. Kotidis E, Papavramidis T, Ioannidis K, Koliakos G, Lazou T, Cheva A, et al. Can chronic intraabdominal hypertension cause oxidative stress to the abdominal wall muscles? An experimental study. The Journal of surgical research. 2012;176(1):102-7.

26. Kotidis EV, Papavramidis TS, Ioannidis K, Cheva A, Lazou T, Michalopoulos N, et al. The effect of chronically increased intra-abdominal pressure on rectus abdominis muscle histology an experimental study on rabbits. The Journal of surgical research. 2011;171(2):609-14.

27. Papavramidis TS, Kotidis E, Ioannidis K, Cheva A, Lazou T, Koliakos G, et al. The effects of chronically increased intra-abdominal pressure on the rabbit diaphragm. Obes Surg. 2012;22(3):487-92.

28. Papavramidis TS, Kotidis E, Ioannidis K, Cheva A, Lazou T, Koliakos G, et al. Diaphragmatic adaptation following intra-abdominal weight changing. Obes Surg. 2011;21(10):1612-6.

29. Cherniack RM, Guenter CA. The efficiency of the respiratory muscles in obesity. Can J Biochem Physiol. 1961;39:1211-22.

30. Wadstrom C, Muller-Suur R, Backman L. Influence of excessive weight loss on respiratory function. Eur J Surg. 1991;157:341-6.

31. Vincken W, Ghezzo H, Cosio MG. Maximal static respiratory pressures in adults: normal values and their relationship to determinants of respiratory function. Bull Eur Physiopathol Respiration. 1987;23(5):435-9.

32. Enright $\mathrm{S}$, Chatham $\mathrm{K}$, Ionescu AA, Unnithan VB, Shale DJ. Inspiratory muscle training improves lung function and exercise capacity in adults with cystic fibrosis. Chest. 2004;126(2):405-11.

33. Carpenter MA, Tockman MS, Hutchinson RG, Davis CE, Heiss G. Demographic and anthropometric correlates of maximum inspiratory pressure: The Atherosclerosis Risk in Communities Study. Am J Respir Crit Care Med. 1999;159(2):415-22. 
34. Hautmann H, Hefele S, Schotten K, Huber HM. Maximal inspiratory mouth pressure (PIMAX) in healthy subjects - what is the lower limit of normal? Respiratory Medicine. 2000;94:689-93.

35. Leech JA, Ghezzo H, Stevens D, Becklake MR. Respiratory pressures and function in young adults. Am Rev Respir Dis. 1983;128:17-23.

36. Enright PL, Kronmal RA, Manolio TA, Schenker MB, Hyatt RE. Respiratory muscle strength in the elderly. Correlates and reference values. Cardiovascular Health Study Research Group. Am J Respir Crit Care Med. 1994;149:430-8.

37. Neder JA, Andreoni S, Lerario MC, Nery LE. Reference values for lung function tests. II. Maximal respiratory pressures and voluntary ventilation. Brazilian journal of medical and biological research $=$ Revista brasileira de pesquisas medicas e biologicas / Sociedade Brasileira de Biofisica [et al]. 1999;32(6):719-27.

38. Harik-Khan RI, Wise RA, Fozard JL. Determinants of maximal inspiratory pressure. The Baltimore Longitudinal Study of Aging. Am J Respir Crit Care Med. 1998;158(5 Pt 1):1459-64.

39. Barbalho-Moulim MC, Miguel GP, Forti EM, Cesar Mde C, Azevedo JL, Costa D. Silicone-ring Roux-en-Y gastric bypass in the treatment of obesity: effects of laparoscopic versus laparotomic surgery on respiration. Obes Surg. 2011;21(2):194-9. 



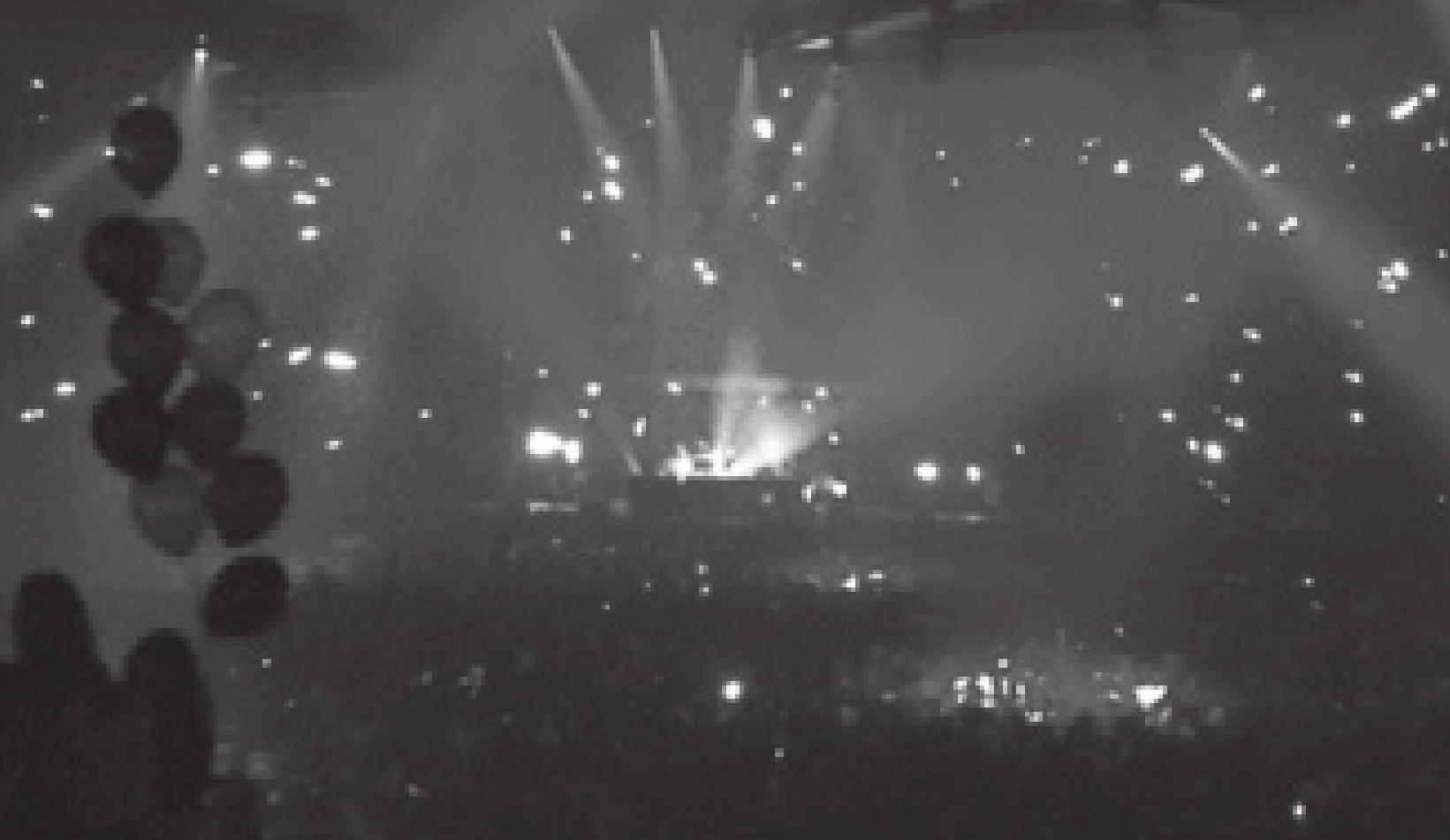

9

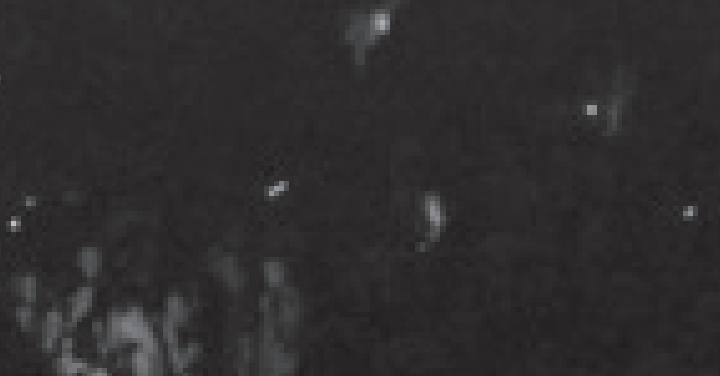




\section{COMPARATIVE ANALYSIS OF RESPIRATORY MUSCLE STRENGTH BEFORE AND AFTER BARIATRIC SURGERY USING FIVE DIFFERENT PREDICTIVE EQUATIONS}

Sjaak Pouwels ${ }^{1,2}$

Marc P. Buise ${ }^{3}$

Frank W.J.M. Smeenk ${ }^{4,5}$ Joep A.W. Teijink ${ }^{1,2}$

Simon W. Nienhuijs ${ }^{1}$

J Clin Anesth. 2016 Aug;32:172-80

1. Department of Surgery, Catharina Hospital, Eindhoven, The Netherlands 2. Department of Epidemiology, CAPHRI Research School, Maastricht University, The Netherlands

3. Department of Anesthesiology, Catharina Hospital, Eindhoven, The Netherlands 4. Department of Respiratory Medicine, Catharina Hospital, Eindhoven, The Netherlands 5. SHE School of Health Professions Education, Maastricht University, The Netherlands 


\section{ABSTRACT}

\section{Background}

Obesity has detrimental effects on general health and respiratory function. This study aimed to evaluate respiratory muscle strength in the morbidly obese population, before and after bariatric surgery, and to compare these estimates with the predictive values using different mathematical equations available.

\section{Methods}

The Maximum Inspiratory Pressure (MIP) was measured at screening and 3, 6 and 9 months postoperative. Predictive values were calculated using five different mathematical equations. Visual inspection of Bland-Altman plots was performed to determine the agreement between the equations studied.

\section{Results}

In total 125 patients were found eligible and 122 patients were available for the final analysis, among them were 104 females and 18 men, with a mean age was $43.02 \pm 11.11$ years and mean BMI was $43.10 \pm 5.25 \mathrm{~kg} / \mathrm{m}^{2}$. In the preoperative period, the predicted MIP according to the Harik-Khan, Neder, Costa and Wilson equation were significantly different compared to the Actual MIP $(\mathrm{p}<0.05)$. The predicted MIP according to the Enright equation was not significantly different $(\mathrm{p}>0.05)$. Postoperatively, there was a significant difference between the MIP values after 3 and 6 months and the predicted MIP values according to Harik Khan, Neder and Enright equation. After 9 months all predicted MIP values were significantly different from the predicted values. Bland Altman analysis showed that the Enright equation was best suitable for predicting the MIP.

\section{Conclusion}

Of the five mathematical equations studied that of Enright and collegues was found best suitable for predicting the MIP in the obese population studied. 


\subsection{INTRODUCTION}

Obesity is the most common chronic metabolic disease worldwide and its prevalence has been strongly increasing. (1) Obesity is a risk factor for various conditions like, cardiovascular disease, type 2 diabetes, rheumatoid arthritis and neoplasms. It is also associated with respiratory dysfunction often leading to obstructive sleep apnoea and obesity hypoventilation syndrome. (1)

\section{OBESITY AND RESPIRATORY FUNCTION}

Over the last years, the effect of obesity on respiratory function has been studied; however there is no consensus as to the physiological mechanisms that lead to respiratory dysfunction and/or complications. Respiratory dysfunction is associated with restrictive lung function disorder. Restrictive pulmonary dysfunction could be explained by the alterations in ventilatory mechanics experienced in the obese. (2)

An increased deposition of fat on the chest wall causes a reduction in thoracic compliance and lung volumes. (3-5) The distorted balance in the interaction between lung, chest wall and diaphragm in this patient population causes impairment in respiratory mechanics and gas exchange. (6)

To evaluate pulmonary function, spirometry is the first step. A pattern of restriction includes: 1. Reduced forced vital capacity (FVC), 2. Reduced forced expiratory volume in one second $\left(\mathrm{FEV}_{1}\right)$, 3. A normal ratio between these two (Tiffeneau Index, which is $\mathrm{FEV}_{1} / \mathrm{FVC}$ ) and 4. Reduced total lung capacity. In case of unexplained respiratory dysfunction, the measurement of maximal inspiratory pressure (MIP) can be of value in the evaluation of the differential diagnosis.

Studies on the behaviour of respiratory muscle strength in morbidly obese patients have showed conflicting results. (4-12) Magnani et al. concluded that neither excess of body mass nor fat distribution on the thorax cage promote respiratory muscle dysfunction. (6) On the other hand respiratory dysfunction is reported in this patient population, which might be due to an increased tensile strength caused by excessive adipose tissue in the thorax region and the abdomen. This can lead to a potential disadvantage in respiratory muscle mechanics. $(4,13)$

\section{ASSESSMENT OF RESPIRATORY PRESSURES}

Assessing the maximal static respiratory pressure generated in the mouth, after complete inhalation and exhalation, carries out the measurement of respiratory muscle strength, divided in the Maximal Inspiratory Pressure (MIP) and Maximal Expiratory Pressure (MEP), 
which are indicative of the strength of the inspiratory and expiratory muscle groups. (14) MIP is a measure of inspiratory muscle strength, whereas MEP measures the strength of the abdominal and intercostal muscles. (15-17) In cardiothoracic surgery, decreased respiratory muscle strength is associated with higher incidence of postoperative pulmonary complications. $(18,19)$ Preoperative inspiratory muscle training can significantly reduce these postoperative pulmonary complications. $(18,19)$ Obese patients have an altered pulmonary function and gas exchange and are therefore prone to postoperative pulmonary complications. $(3,20)$

\section{PREDICTIVE EQUATIONS}

Measuring MIP values can give valuable information about the respiratory muscle strength in the obese and can be used as a reference value for training of respiratory muscle strength. To set adequate training goals, the use of predictive equations is mandatory to set training goals. (14, 16, 21-23) However, the current predictive equations and reference values (of MIP and MEP) are based on the normal populations and less is known about the recommended reference values for respiratory muscle strength in the morbidly obese population. Also there is no consensus on the best suitable mathematical equation for predicting respiratory muscle strength in this population.

\section{Objectives}

- $\quad$ Primary objective of this study is to identify which of the existing predictive equations is the most suitable for the morbidly obese patient.

- Secondary objective is to evaluate respiratory muscle strength in the morbidly obese population, before and after bariatric surgery, and to compare these estimates with the predictive values using different mathematical equations available.

\section{Hypotheses}

- Predictive equations that include anthropometric variables will either predict correctly or will overestimate the MIP.

- $\quad$ Predictive equations that without anthropometric variables will underestimate the MIP. 


\subsection{METHODS}

This study was approved by the Medical Ethics Committee of the Catharina Hospital Eindhoven and adheres the principles of the declaration of Helsinki. Informed consent was obtained of all patients included prior to the study.

\section{PATIENT POPULATION}

A multidisciplinary team screened all patients preoperatively, consisting of a physician assistant, a psychologist, a nutritionist and a surgeon. Eligibility criteria for surgery were if patients had a BMI of $40 \mathrm{~kg} / \mathrm{m}^{2}$ or higher, or a BMI between 35 and $40 \mathrm{~kg} / \mathrm{m}^{2}$ with significant co-morbidities, with serious attempts to lose weight in the past. Co-morbidities were considered significant when medication had to be used, or if continuous positive airway pressure (CPAP) had to be used in case of OSAS. The indication for a bariatric procedure was made in a multidisciplinary consultation, taking into account the patients' preference, age, poly-pharmacy, reflux complaints, body composition in relation to BMI and bowel diseases. Patients with pre-existing pulmonary and/or neuromuscular diseases were excluded from this study.

\section{ANAESTHESIA AND PERIOPERATIVE CARE}

All anaesthesia given and perioperative care was according to fast track (FT) protocol, previously described. $(24,25)$ Patients received 5000 units of low-molecular weight heparin as thrombosis prophylaxis. Premedication consisted of only acetaminophen $1000 \mathrm{mg}$. Anaesthesia was induced using a combination of piritramide $(0.2-0.3 \mathrm{mg} / \mathrm{kg})$, propofol $(2 \mathrm{mg} / \mathrm{kg})$ and suxamethoniumchloride $(1-1.5 \mathrm{mg} / \mathrm{kg})$. Anaesthesia maintainance was conducted with desflurane $(6.0 \mathrm{vol} \%)$ in combination with remifentanil $(5-15 \mu \mathrm{g} / \mathrm{kg} / \mathrm{hr})$ (26). By standardizing discontinuation of desflurane and remifentanil upon notification of the surgeon during application of the last sutures, patients were directly extubated after the procedure and able to replace themselves to their bed. Fluid administration was restricted to 1 litre of lactated Ringers intraoperative and to a maximum of 1 litre of lactated Ringers postoperatively (27). Postoperatively analgesia consisted of parecoxib (40mg) once followed by acetaminophen (1000mg four times daily) and tramadol (100mg). In case of inadequate analgesia (NRS score $>4$ ) piritramide $(0.2-0.3 \mathrm{mg} / \mathrm{kg}$, on average about $20 \mathrm{mg}$ i.m.) was administered for pain relieve. After approximately one hour in the postanaesthesia care unit, all patients (including the extremely obese) were transferred to the surgical ward. On the ward, patients are directly mobilized as part of thrombosis prophylaxis. $(24,25)$ 


\section{PREDICTIVE EQUATIONS}

Harik-Khan Equation (16):

Women: MIP $=171-(0.694 \mathrm{x}$ age $)+(0.861 \mathrm{x}$ body mass $(\mathrm{kg}))-(0.743 \mathrm{x}$ height $(\mathrm{cm}))$

Men: MIP $=126-(1.028 \times$ age $)+(0.343 \times$ weight $(\mathrm{kg}))$

Neder Equation (22):

Women: MIP $=(-0.49 x$ age $)+110.4$

Men: MIP $=(-0.80 \mathrm{x}$ age $)+155.3$

Costa Equation (14):

Women: MIP $=(-0.46 \times$ age $)+74.25$

Men: MIP $=(-1.24 \mathrm{x}$ age $)+232.37$

Enright Equation (21):

Women: $\mathrm{MIP}=(0.133 \mathrm{x}$ weight $(\mathrm{kg}))-(0.805 \mathrm{x}$ age $)+96$

Men: $\mathrm{MIP}=(0.131 \mathrm{x}$ weight $(\mathrm{kg}))-(1.27 \mathrm{x}$ age $)+153$

Wilson Equation (23):

Women: MIP $=-43+(0.71 \times$ height $(\mathrm{cm}))$

Men: MIP $=142-(1.03 \times$ age $)$

\section{DATA COLLECTION}

Data were collected prospectively using an online registry (Patients Outcome Measurement Tool). The patients who got approval between the $3^{\text {rd }}$ of October 2011 and the $3^{\text {rd }}$ of May 2012 were included in this study as during this period the inspiratory muscle function was measured.

\section{OUTCOME MEASURES}

Respiratory muscle strength was assessed by measuring the maximal inspiratory pressure (MIP) with a digital mouth pressure meter $\left( \pm 300 \mathrm{~cm} \mathrm{H}_{2} \mathrm{O}\right)$. This procedure was performed by a trained independent physiotherapist according to the ATS/ERS protocol $(28,29)$ and took place took place before bariatric surgery and 3, 6 and 9 months postoperatively. The manometer (Micro RPM ${ }^{\circledast}$, Carefusion, USA) was calibrated in accordance with the manufacturer's recommendations. Predictive MIP values were calculated using the earlier mentioned five different predictive equations. The predicted MIP values were compared with each other, with the actual MIP and the postoperative MIP values. Patient characteristics and weight loss profiles were obtained from medical charts of each patient. 


\section{STATISTICAL ANALYSIS}

Continuous variables were presented as mean \pm standard deviation (SD). Categorical variables were presented as frequency with percentages. Data distribution was verified using the Shapiro-Wilk test. To compare the MIP values the paired t-test was used for parametric data or the Mann-Whitney U test was used, for non-parametric data. To compare the MIP values at different times (predicted, preoperatively, and after 3, 6 and 9 months after surgery), repeated measures ANOVA (parametric data) was used or the Kruskall Wallis test (nonparametric data).

Visual inspection of Bland-Altman plots was performed to determine the agreement between the equations studied. Values of $\mathrm{p}<0.05$ were considered statistically significant. Statistical Package for Social Sciences (SPSS, Chicago, IL, USA Version 20.0) was used to prepare the database and for statistical analysis.

\subsection{RESULTS}

\section{PATIENTS AND PROCEDURES}

In total 125 were found eligible for this study and 122 patients were included in this study, among them were 104 females and 18 men. Three patients did not meet the inclusion criteria because of pre-existing neuromuscular and/or pulmonary diseases (see Figure 7.1 for the CONSORT diagram).

The mean age was $43.02 \pm 11.11$ years, mean height was $169.03 \pm 8.42 \mathrm{~cm}$, mean weight was $123.46 \pm 19.45 \mathrm{~kg}$ and mean BMI was $43.10 \pm 5.25 \mathrm{~kg} / \mathrm{m}^{2}$. Half the patients underwent a sleeve gastrectomy $(n=60 ; 49.0 \%)$ and the other half received a gastric bypass $(n=62 ; 51.0 \%)$. Postoperatively, the mean weight and BMI 3, 6 and 9 months after surgery were respectively $105.1 \pm 18.1 \mathrm{~kg}$ (BMI: $\left.37.0 \pm 5.3 \mathrm{~kg} / \mathrm{m}^{2}\right), 91.7 \pm 15.2 \mathrm{~kg}\left(\right.$ BMI: $32.0 \pm 6.7 \mathrm{~kg} / \mathrm{m}^{2}$ ) and $82.8 \pm 16.8$ $\mathrm{kg}$ (BMI: $29.0 \pm 7.2 \mathrm{~kg} / \mathrm{m}^{2}$ ). Table 7.1 gives an overview of the preoperative and postoperative characteristics.

\section{PREDICTED MIP AND THE PREOPERATIVE MEASURED MIP}

In the preoperative period, the predicted MIP according to the Harik-Khan (16), Neder (22), Costa (14) and Wilson (23) equation were significantly different compared to the Actual MIP $(\mathrm{p}<0.05)$ (Table 7.2). Only the predicted MIP according to the Enright (21) equation was not significantly different compared to the Actual MIP ( $p>0.05)$. 


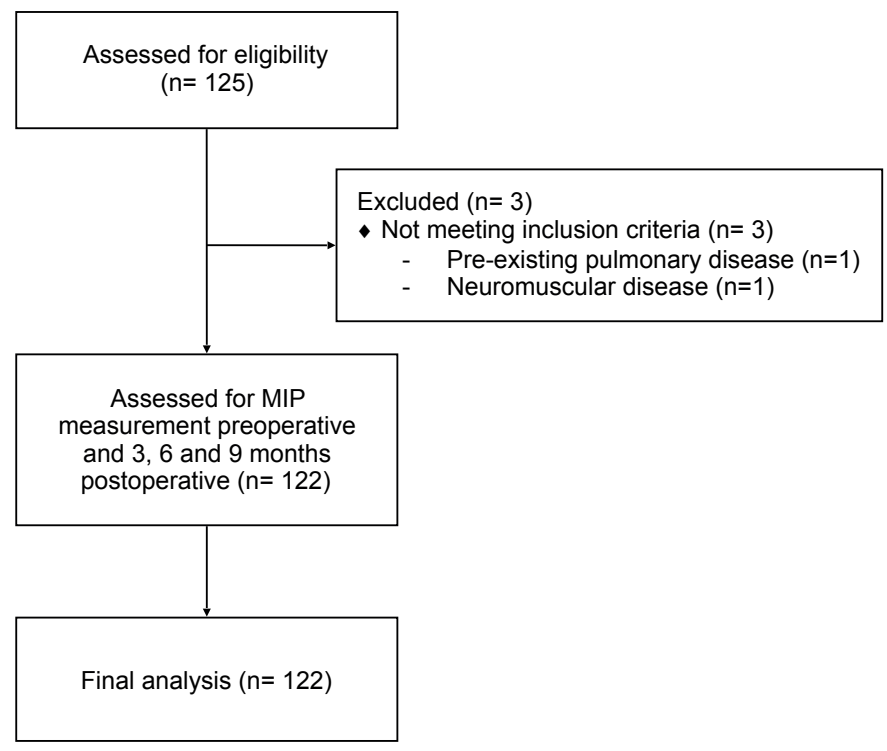

FIGURE 7.1: CONSORT diagram for the study

\section{PREDICTED MIP AND MIP 3, 6, 9 MONTHS AFTER SURGERY}

In the postoperative period, the MIP was $75.58 \pm 26.10,81.61 \pm 28.12$ and $85.69 \pm 27.97 \mathrm{~cm}$ $\mathrm{H}_{2} \mathrm{O}$ respectively 3,6 and 9 months after surgery (Table 7.3). The was a significant difference between the MIP values after 3 and 6 months and the predicted MIP values according to Harik Khan (16), Neder (22) and Enright (21) equation. After 9 months all predicted MIP values were significantly different from the predicted values.

\section{BLAND-ALTMAN ANALYSIS}

For the actual MIP preoperative, the statistical graph analysis was performed between the preoperative measured values and those predicted by the used equations. (Figure 7.2 and 7.3) The Harik-Khan equation showed a mean difference of $-19.72 \mathrm{~cm} \mathrm{H}_{2} \mathrm{O}$ and an agreement interval of -125.80 to $52.11 \mathrm{~cm} \mathrm{H}_{2} \mathrm{O}$. The obtained preoperative values from the Wilson equation showed a mean difference of $23.18 \mathrm{~cm} \mathrm{H}_{2} \mathrm{O}$ and an agreement interval of -37.80 to $87.99 \mathrm{~cm} \mathrm{H}_{2} \mathrm{O}$. The mean difference of the Enright equation was $-0.69 \mathrm{~cm} \mathrm{H}_{2} \mathrm{O}$ with an agreement interval from -90.41 to $68.71 \mathrm{~cm} \mathrm{H}_{2} \mathrm{O}$. The mean differences and agreement intervals of the Neder and Costa equation were $8.21 \mathrm{~cm} \mathrm{H}_{2} \mathrm{O}$ (agreement interval -60.30 to $73.12 \mathrm{~cm} \mathrm{H}_{2} \mathrm{O}$ ) and $29.30 \mathrm{~cm} \mathrm{H}_{2} \mathrm{O}$ (agreement interval -119.77 to $107.83 \mathrm{~cm} \mathrm{H}_{2} \mathrm{O}$ ). 


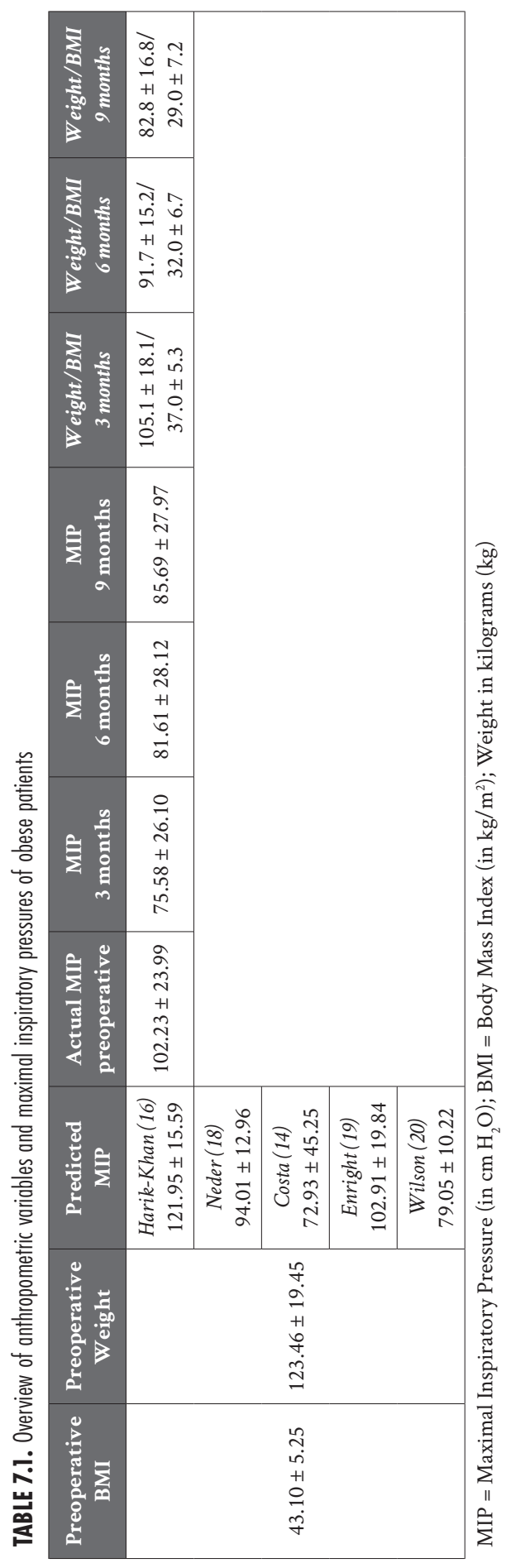


TABLE 7.2. Maximal inspiratory pressures of obese patients 3,6 and 9 months postoperatively and the preoperative predicted values using five different predictive equations

\begin{tabular}{|c|c|c|c|c|c|c|}
\hline MIP 3 mont hs* & MIP 6 months* & MIP 9 months* & Predicted MIP & \multicolumn{3}{|c|}{ P-value } \\
\hline \multirow{5}{*}{$75.58 \pm 26.10$} & \multirow{5}{*}{$81.61 \pm 28.12$} & \multirow{5}{*}{$85.69 \pm 27.97$} & $\begin{array}{c}\text { Harik-Khan }(16)^{*} \\
121.95 \pm 15.59\end{array}$ & $0.001^{\mathrm{A}}$ & $0.001^{\mathrm{B}}$ & $0.001^{\mathrm{C}}$ \\
\hline & & & $\begin{array}{c}\text { Neder }(18)^{*} \\
94.01 \pm 12.96\end{array}$ & $0.001^{\mathrm{A}}$ & $0.001^{\mathrm{B}}$ & $0.001^{\mathrm{C}}$ \\
\hline & & & $\begin{array}{c}\text { Costa }(14)^{*} \\
72.93 \pm 45.25\end{array}$ & $0.529^{A}$ & $0.044^{\mathrm{B}}$ & $0.004^{\mathrm{C}}$ \\
\hline & & & $\begin{array}{c}\text { Enright }(19)^{*} \\
102.91 \pm 19.84\end{array}$ & $0.001^{\mathrm{A}}$ & $0.001^{\mathrm{B}}$ & $0.001^{\mathrm{C}}$ \\
\hline & & & $\begin{array}{c}\text { Wilson (20)* } \\
79.05 \pm 10.22\end{array}$ & $0.143^{\mathrm{A}}$ & $0.311^{\mathrm{B}}$ & $0.009^{\mathrm{C}}$ \\
\hline
\end{tabular}

* in $\mathrm{cm} \mathrm{H}_{2} \mathrm{O}$

A compared with MIP 3 months

${ }^{\text {B }}$ compared with MIP 6 months

${ }^{\mathrm{C}}$ compared with MIP 9 months 

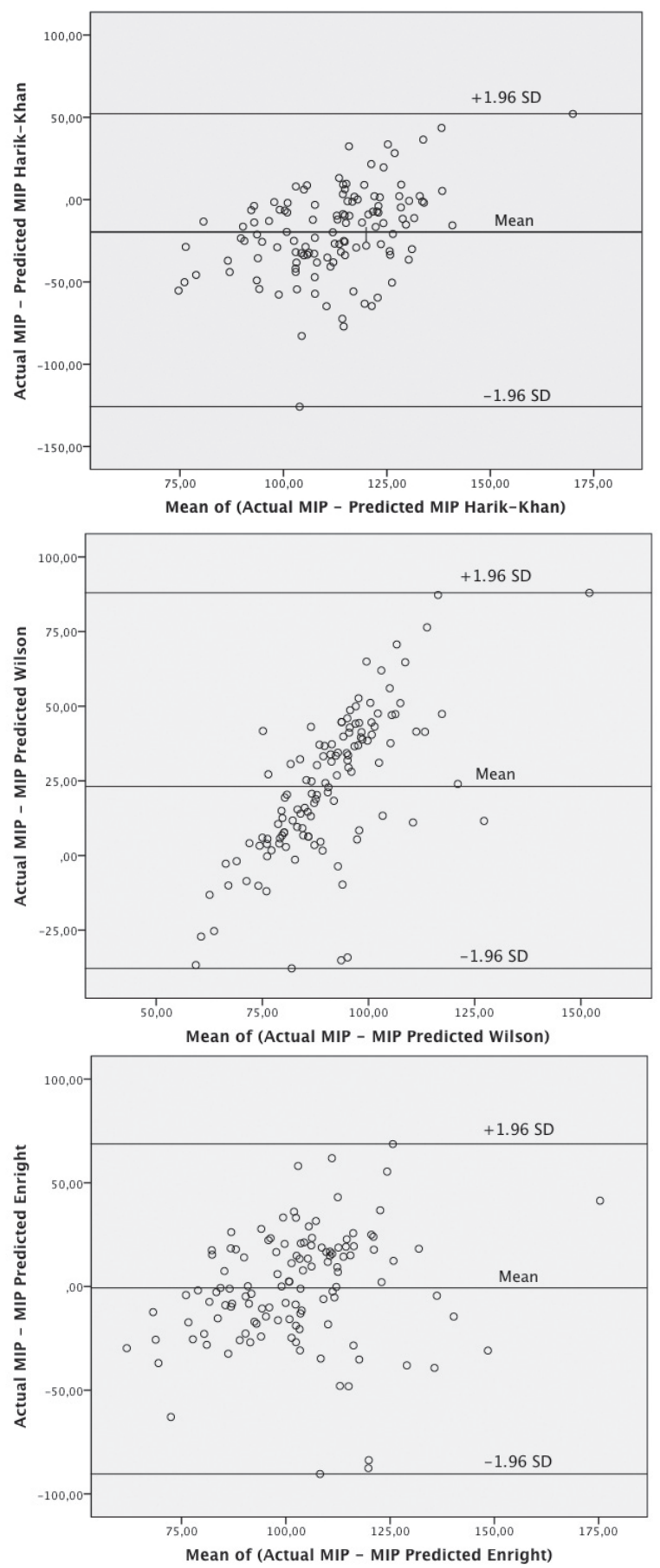

FIGURE 7.2: Concordance between mean values of MIP (actual preoperative and predicted) for Harik-Khan, Wilson and Enright. 

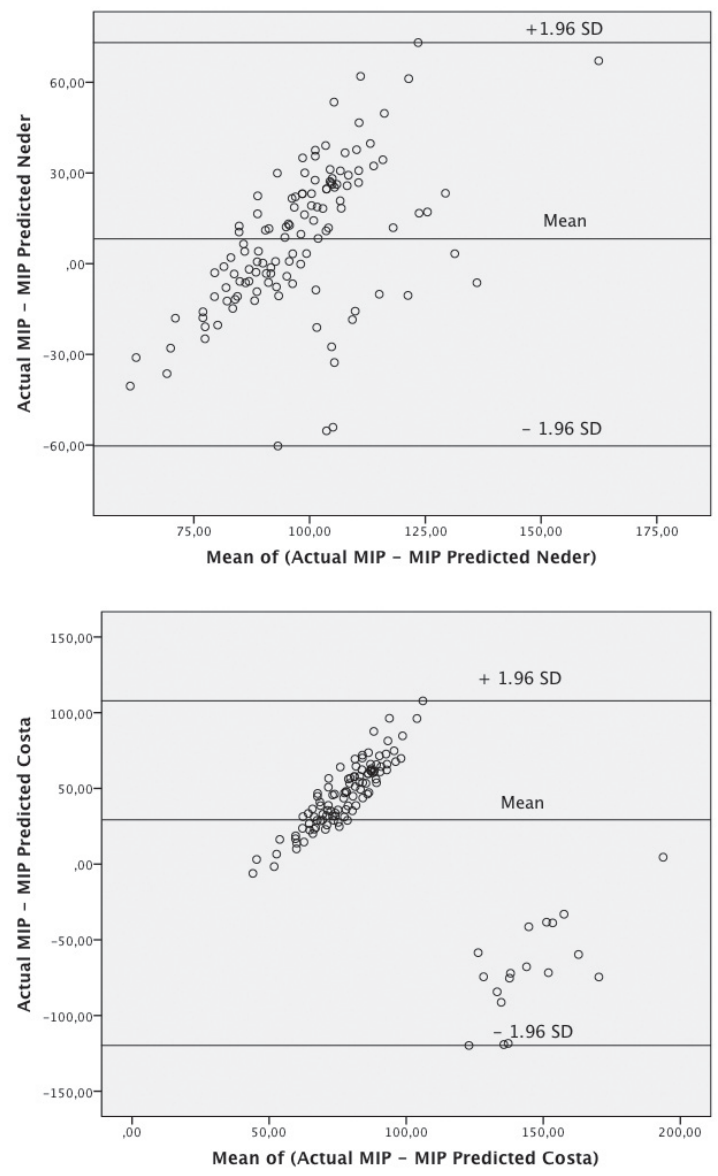

FIGURE 7.3. Concordance between mean values of MIP (actual preoperative and predicted) for Neder and Costa.

\subsection{DISCUSSION}

The measurement of the MIP is a simple and non-invasive clinical procedure for the determining the inspiratory muscle strength in healthy people and in patients with pulmonary, neuromuscular or cardiovascular disease. (30-33) In the latter group, MIP is indicative of ventilatory capacity and the development of respiratory insufficiency (31), and can be useful in assessing the degree of abnormality and in monitoring inspiratory muscle weakness in individual patients over time $(30,32)$ MIP can also be helpful in evaluating the success of weaning patients from mechanical ventilators $(16,31)$ and in predicting the outcome of cardiac transplantation surgery in patients with chronic heart failure. (33) 
A recent study by Pazzianotto-Forti and colleagues compared three predictive equations (Harik-Khan (16), Neder (22) and Costa (14)) to investigate the behaviour of respiratory muscle strength in morbidly obese women. (17) They compared a cohort of 30 morbidly obese women with 30 normal weight subjects. They measured a mean MIP of $87.83 \pm 21.40$ $\mathrm{cm} \mathrm{H}_{2} \mathrm{O}$ in the obese group and $72 \pm 15.23 \mathrm{~cm} \mathrm{H} \mathrm{H}_{2} \mathrm{O}$ in the normal weight group. In the obese group, the predicted MIPs (using the Harik-Khan, Neder and Costa equation) were respectively $130.71 \pm 11.98,94.55 \pm 3.05$ and $50.37 \pm 2.86 \mathrm{~cm} \mathrm{H}_{2} \mathrm{O}$. The Harik-Khan equation significantly overestimated the MIP, the Costa equation significantly underestimated the MIP and there was no difference in the MIP measured and predicted by the Neder equation. (17) Regarding the normal weight group, there was no difference in the measured MIP and and predicted by the Harik-Khan equation (respectively $72 \pm 15.23$ and $79.76 \pm 5.31 \mathrm{~cm} \mathrm{H}_{2} \mathrm{O}$ ). The MIP predicted by the Neder equation was significantly higher and the MIP predicted by the Costa equation was significantly lower (respectively $95.47 \pm 2.57$ and $60.23 \pm 2.41 \mathrm{~cm} \mathrm{H}_{2} \mathrm{O}$ ). Pazzianotto-Forti and colleagues concluded the Harik-Khan equation was the most suitable for predicting the MIP in the morbidly obese, because it's the only formula that inclus the body mass and height. (17)

In our cohort of 122 morbidly obese patients, the preoperative measured MIP was significantly different with the MIP values predicted by the Harik-Khan (16), Neder (22), Costa (14) and Wilson equation (23). The Wilson (23), Costa (14) and Neder (22) equation significantly underestimated the MIP and the Harik-Khan (16) overestimated the MIP. The MIP value predicted by the equation of Enright and colleagues was not significantly different from the preoperative measured MIP. These findings support our hypotheses. Our results regarding the Harik-Khan and Costa equation are supported by the study by Pazzianotto-Forti (17) Regarding the Neder equation Pazzianotto-Forti et al. (17) found no significant difference between the measured and predicted MIP, where we found a significant underestimation.

When we compared the postoperative measured MIP values (after 3, 6, 9 months) with the predicted values, several important facts become visible. When comparing the MIP values 3 months and 6 months after surgery, the following is seen: the Harik-Khan (16), Neder (22) and Enright (21) equation significantly overestimate the MIP; the predicted value of the Costa (14) equation was not significantly different (after 3 months), but after 6 months there was a significant underestimation; the predicted MIP by Wilson (23) equation did not significantly differ (after 3 and 6 months). After 9 months, the Harik-Khan (16), Neder (22) and Enright (21) equation showed a significant overestimation of the MIP and the Costa (14) and Wilson (23) equation showed a significant underestimation.

Explanations for the differences in results between our study and the study by PazzianottoForti are the following: Pazzianotto-Forti investigated Brazilian patients and we focussed on 
Caucasian patients, secondly the sample size of both studies differed (60 patients versus 122 patients) and thirdly we focussed on the postoperative measures and Pazzianotto-Forti did not. (17)

Four conclusions can be drawn for the clinical use of the predictive equations from our study. First of all, there were significant differences between the measured MIP values and the values predicted. Only there was no significant difference between the measured MIP values and the values predicted by the equation of Enright and colleagues. (21) Secondly, none of the mathematical equations used were able to precisely predict the MIP, which confirms the study's hypothesis that in the morbidly obese population it is difficult to predict respiratory muscle strength. Thirdly, the greatest agreement was found between de measured preoperative MIP and the predicted values using the Enright (21) equation (mean difference -0.69; agreement interval -90.41 to 68.71 ). Therefore, this equation was selected in this study as the most reliable for predicting the MIP in the morbidly obese. However when looking more closely to the distribution patterns on the Bland-Altman plots, two important aspects need attention. Bland-Altman plots are a comparison method which pairs the differences between measures taken using two devices to the mean of the two values, and therefore avoiding mathematical traps and statistical misconceptions. (34) But they need to be analysed with caution and precision. (34) First there are no values above and below the $95 \%$ confidence intervals. This can be a result of our misbalance of included males and females included study (there are more females than males included in this study, and there are known differences between the MIP values between males and females $(6,16,17,21))$, however this does not clarify this phenomenon totally. Also race can be an important factor to clarify the differences in MIP values and also can clarify the distribution in the Bland-Altman plots. Secondly, an interesting fact is that there is no equal spreading of values around the mean difference, which is uncommon. In most cases, there is an equal spreading around the mean difference line in Bland-Altman. (34)

In studies investigating the respiratory muscle strength in the morbidly obese, several difficulties arise, as mentioned by Bruschi and colleagues. (32) Great variety in results were mentioned, due to variability to different methodology; such as the type of mouthpiece, the number of manoeuvres performed, body position and the differences in the populations studied. $(17,32,35)$

Interesting finding in our current study is that the MIP after 3 months decreased and after 6 and 9 months gradually increases. Three main factors have to be taken into account, muscle function of the respiratory muscles, abdominal muscles and the lung compliance. (36) The main reason is probably the decrease of fat mass ( 3 months after surgery) and adaptation of the respiratory muscle function ( 6 and 9 months after surgery) (36) 
In current Dutch bariatric practice, the clinical relevancy of the MIP measurement is questionable. This is because all bariatric surgical procedures are performed laparoscopically and therefore pulmonary complications are rarely seen. (1) But in other diseases (especially neuromuscular diseases, cardiac surgery or severe COPD) the MIP measurement might helpful for determining the clinical stadium of disease or to determine whether a patient is prone to postoperative pulmonary complications. A randomised clinical trial by Hulzebos et al. (18) investigated whether inspiratory muscle training is effective in reducing postoperative complications in patients scheduled for elective coronary artery bypass grafting (CABG) surgery. Inspiratory muscle training is effective in reducing postoperative pulmonary complications according to this trial. (18) In general, obesity is an increasing problem. (37) To determine the adequate training goal, there is increasing need for a mathematical equation which can adequately predict the respiratory muscle strength and therefore to set achievable training goals.

\section{STUDY LIMITATIONS AND FUTURE RESEARCH}

Our study has several limitations that have to be taken into account. First, our male/female equilibrium was not equal. Secondly, our study lacks of body composition analysis, which can give a clearer view of the influence of fat depositions on the measured MIP values. Also ethnic differences in MIP have to be studied, but our sample was too small to perform such an analysis.

To get more insight in the respiratory function of the morbidly obese and the influence of bariatric surgery on respiratory muscle function several function parameters have to be studied. As mentioned earlier, spirometry is the first step for testing pulmonary function. A comparative analysis between spirometric variables and maximal inspiratory/expiratory pressures can give more insight in the pulmonary physiology of the morbidly obese.

\subsection{CONCLUSION}

Of the five mathematical equations studied, that of Enright et al. (21) seems to be the most suitable for calculating the MIP reference values of the morbidly obese. Further research is necessary to gain more insight in the pulmonary function of the morbidly obese, the influence of bariatric surgery on the pulmonary function and to assess the MIP values in larger samples to develop a predictive equation for the obese. 


\subsection{REFERENCES}

1. WHO, "Obesity: preventing and Managing the global epidemic," Report of a WHO Consultation. 2000;894(1-12):1-253.

2. Melo LC, Silva MA, Calles AC. Obesity and lung function: a systematic review. Einstein (Sao Paulo, Brazil). 2014;12(1):120-5.

3. Hamoui N, Anthone G, Crookes PF. The value of pulmonary function testing prior to bariatric surgery. Obesity Surgery. 2006;16(12):1570-3.

4. Weiner P, Waizman J, Weiner M, Rabner M, Magadle R, Zamir D. Influence of excessive weight loss after gastroplasty for morbid obesity on respiratory muscle performance. Thorax. 1998;53(1):39-42.

5. Sarikaya S, Cimen OB, Gokcay Y, Erdem R. Pulmonary function tests, respiratory muscle strength, and endurance of persons with obesity. Endocrinologist. 2003;13(2):136-41.

6. Magnani KL, Cataneo AJM. Respiratory muscle strength in obese individuals and influence of upper-body fat distribution. Sao Paulo Med J. 2007;125(4):215-9.

7. Wadstrom C, Muller-Suur R, Backman L. Influence of excessive weight loss on respiratory function. A study of obese patients following gastroplasty. Eur J Surg. 1991;157(5):341-6.

8. Kelly TM, Jensen RL, Elliott CG, Crapo RO. Maximum respiratory pressures in morbidly obese subjects. Respiration. 1988;54(2):73-7.

9. Sampson MG, Grassino AE. Load compensation in obese patients during quiet tidal breathing. J Appl Physiol. 1983;55(4):1269-76.

10. Black LF, Hyatt RE. Maximal respiratory pressures: normal values and relationship to age and sex. Am Rev Respir Dis. 1969;99:696-702.

11. Parreira VF, Matos CMP, Athayde FTS, Moraes KS, Barbosa KS, Britto RR. Evolution of respiratory muscle strength in post-operative gastroplasty. Rev Bras Fisioter. 2012;16(3):225-30.

12. Cherniack RM, Guenter CA. The efficiency of the respiratory muscles in obesity. Can J Biochem Physiol. 1961;39:1211-22.

13. O’Brien PE, Dixon JB. The extent of the problem of obesity. Am J Surg. 2002;184(6B):4-8.

14. Costa D, Goncalves HA, Lima LP, Ike D, Cancelliero KM, Montebelo MI. New reference values for maximal respiratory pressures in the Brazilian population. Jornal brasileiro de pneumologia : publicacao oficial da Sociedade Brasileira de Pneumologia e Tisilogia. 2010;36(3):306-12.

15. Barbalho-Moulim MC, Miguel GP, Forti EM, Campos Fdo A, Costa D. Effects of preoperative inspiratory muscle training in obese women undergoing open bariatric surgery: respiratory muscle strength, lung volumes, and diaphragmatic excursion. Clinics (Sao Paulo, Brazil). 2011;66(10):1721-7.

16. Harik-Khan RI, Wise RA, Fozard JL. Determinants of maximal inspiratory pressure. The Baltimore Longitudinal Study of Aging. Am J Respir Crit Care Med. 1998;158(5 Pt 1):1459-64.

17. Pazzianotto-Forti EM, Peixoto-Souza FS, Piconi-Mendes C, Rasera-Junior I, Barbalho-Moulim M. Behavior of respiratory muscle strength in morbidly obese women by using different predictive equations. Rev Bras Fisioter. 2012;16(6):479-86. 
18. Hulzebos EH, Helders PJ, Favie NJ, De Bie RA, Brutel de la Riviere A, Van Meeteren NL. Preoperative intensive inspiratory muscle training to prevent postoperative pulmonary complications in high-risk patients undergoing CABG surgery: a randomized clinical trial. Jama. 2006;296(15):1851-7.

19. Hulzebos EH, Smit Y, Helders PP, van Meeteren NL. Preoperative physical therapy for elective cardiac surgery patients. The Cochrane database of systematic reviews. 2012;11:CD010118.

20. Jubber AS. Respiratory complications of obesity. Journal of Clinical Practice. 2004;58(6):573-80.

21. Enright PL, Kronmal RA, Manolio TA, Schenker MB, Hyatt RE. Respiratory muscle strength in the elderly. Correlates and reference values. Cardiovascular Health Study Research Group. Am J Respir Crit Care Med. 1994;149:430-8.

22. Neder JA, Andreoni S, Lerario MC, Nery LE. Reference values for lung function tests. II. Maximal respiratory pressures and voluntary ventilation. Brazilian journal of medical and biological research $=$ Revista brasileira de pesquisas medicas e biologicas / Sociedade Brasileira de Biofisica [et al]. 1999;32(6):719-27.

23. Wilson SH, Cooke NT, R.H.T. E, Spiro SG. Predicted normal values for maximal respiratory pressures in caucasian adults and children. Thorax. 1984;39:535-8.

24. van Wezenbeek MR, Pouwels S, Buise MP, Smulders JF, Nienhuijs SW, van Montfort G. Conventional care versus fast track care in an unselected group of patients undergoing revisional bariatric surgery. in press.

25. Vreeswijk SJM, Van Rutte P, Nienhuijs S, Bouwman RA, Smulders JF, Buise MP. The safety and efficiency of a fast-track protocol in gastric sleeve surgery. in press.

26. Chand B, Gugliotti D, Schauer P, Steckner K. Perioperative management of the bariatric surgery patient: focus on cardiac and anesthesia considerations. Cleve Clin J Med. 2006;73(Suppl 1):S51-6.

27. Lemanu DP, Srinivasa S, Singh PP, Johannsen S, MacCormick AD, Hill AG. Optimizing perioperative care in bariatric surgery patients. Obes Surg. 2012;22(6):979-90. doi: 10.1007/ s11695-012-0648-6.

28. Celli BR, MacNee W. Standards for the diagnosis and treatment of patients with COPD: a summary of the ATS/ERS position paper. The European respiratory journal. 2004;23(6):932-46.

29. Standards for the diagnosis and care of patients with chronic obstructive pulmonary disease. American Thoracic Society. Am J Respir Crit Care Med. 1995;152(5 Pt 2):S77-121.

30. McElvaney G, Blackie S, Morrison NJ, Wilcox PG, Fairbarn MS, Pardy RL. Maximal static respiratory pressures in the normal elderly. Am Rev Respir Dis. 1989;139(1):277-81.

31. Karvonen J, Saarelainen S, Nieminen MM. Measurement of respiratory muscle forces based on maximal inspiratory and expiratory pressures. Respiration. 1994;61(1):28-31.

32. Bruschi C, Cerveri I, Zoia MC, Fanfulla F, Fiorentini M, Casali L, et al. Reference values of maximal respiratory mouth pressures: a population-based study. Am Rev Respir Dis. 1992;146(3):790-3.

33. Ambrosino N, Opasich C, Crotti P, Cobelli F, Tavazzi L, Rampulla C. Breathing pattern, ventilatory drive and respiratory muscle strength in patients with chronic heart failure. The European respiratory journal. 1994;7(1):17-22. 
34. Olofsen E, Dahan A, Borsboom G, Drummond G. Improvements in the application and reporting of advanced Bland-Altman methods of comparison. Journal of clinical monitoring and computing. 2015;29(1):127-39.

35. Peixoto-Souza FS, Piconi-Mendes C, Baltieri L, Rasera-Junior I, Barbalho-Moulim MC, Lima Montebelo MI, et al. Lung age in women with morbid obesity. Revista da Associacao Medica Brasileira (1992). 2013;59(3):265-9.

36. Pouwels S, Kools-Aarts M, Said M, Teijink JA, Smeenk FWJM, Nienhuijs SW. Effects of bariatric surgery on inspiratory muscle strength. in press.

37. Ng M, Fleming T, Robinson M, Thomson B, Graetz N, Margono C, et al. Global, regional, and national prevalence of overweight and obesity in children and adults during 1980-2013: a systematic analysis for the Global Burden of Disease Study 2013. Lancet. 2014;384(9945):766-81. 



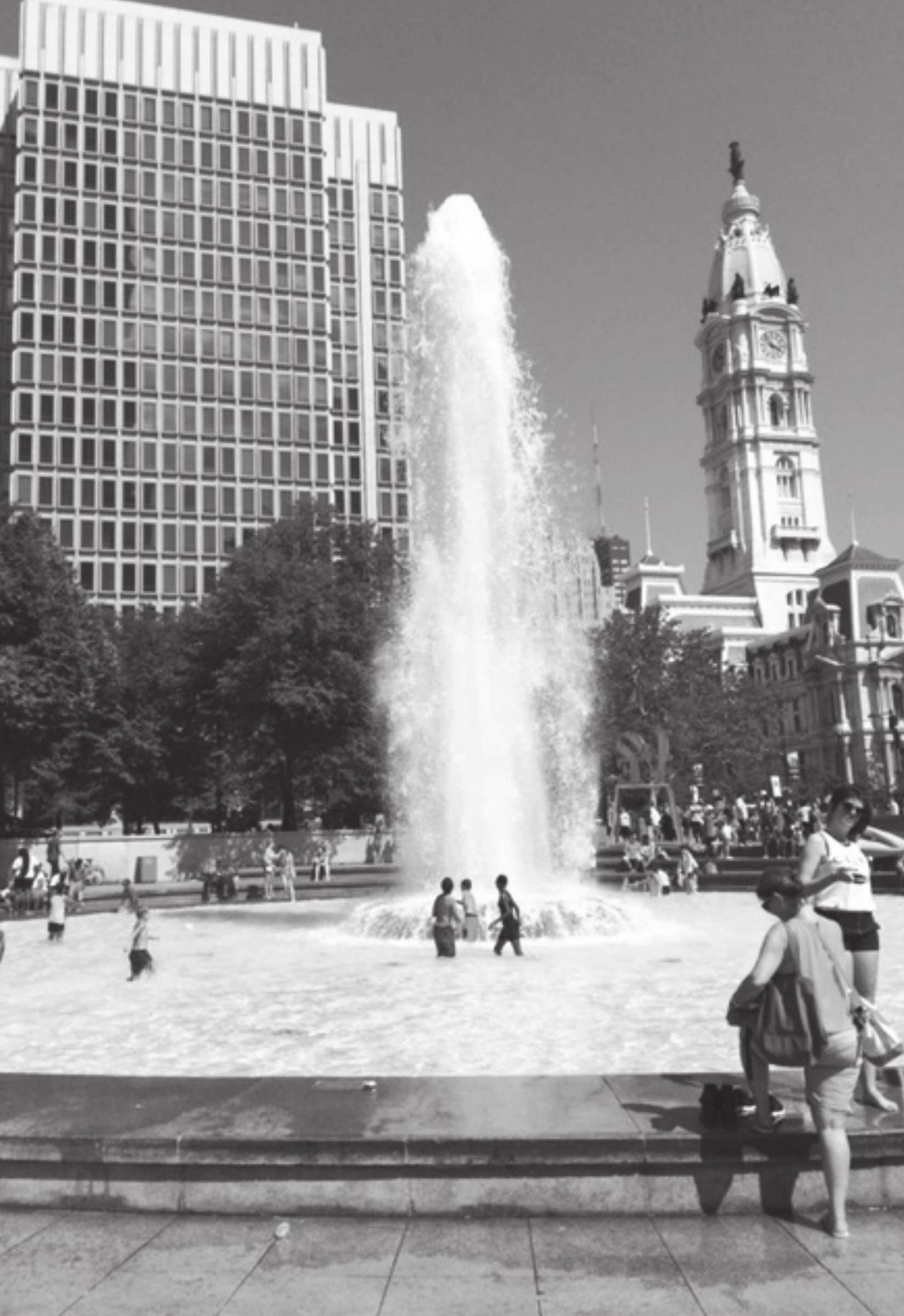




\section{ASPECTS OF EXERCISE BEFORE AND AFTER BARIATRIC SURGERY: A SYSTEMATIC REVIEW}

Sjaak Pouwels ${ }^{1,2}$

Marjon Wit ${ }^{3}$

Joep A.W. Teijink ${ }^{1,2}$

Simon W. Nienhuijs ${ }^{1}$

Obes Facts 2015; 8 (2): 132-146

1. Department of Surgery, Catharina Hospital, Eindhoven, The Netherlands 2. Department of Epidemiology, CAPHRI Research School, Maastricht University, The Netherlands 3. Department of Physiotherapy, Catharina Hospital, Eindhoven, The Netherlands 


\section{ABSTRACT}

\section{Background}

Bariatric surgery has a considerable effect on weight loss. A positive relation of exercise and weight loss has been described before. However, the mode of exercise and its timing pre- or postoperative or a combination remains unclear.

\section{Methods}

A multi database search was conducted. Identified articles were reviewed on description of exercise, timing around a bariatric intervention and outcome. Methodological quality of the included studies was rated using the Physiotherapy Evidence Database scale. A Cohen's kappa score assessed the level of agreement. Outcome measurements were improvement of anthropometric and physical fitness variables, operation related complications, weight regain and quality of life.

\section{Results}

A total of eight prospective studies were included. Four focussed on training before and four on training after a bariatric procedure. Details of exercises varied from 45 minutes treadmill up to full descriptive programs. Supervision was frequently included. Significant improvement was encountered for biometric results physical fitness variables.

\section{Conclusion}

In the majority of reports on exercising in a (future) bariatric population, a positive effect on anthropometrics, cardiovascular risk factors and physical fitness was described. However, the results were not unanimous, with a wide range of exercise programs and peri-operative timing and therefore hampering adequate practical guidance. 


\subsection{INTRODUCTION}

Obesity is a major contributor to a wide variety of diseases, including diabetes, hypertension, dyslipidaemia and obstructive sleep apnoea syndrome. $(1,2)$ Over the last decade's dietary and exercise interventions had increasing public health and governmental attention. Nevertheless, the proportion of the population with morbid obesity gained every year. (3) The only treatment with a longstanding effect is bariatric surgery. $(4,5)$ However, these interventions are no universal remedy and a considerable proportion of patients has insufficient weight loss or regain in weight over time after the operation. (6)

The key elements for durable weight-loss are pre-operative screening, an appropriate algorithm of bariatric techniques and most important, a strict regime of follow-up. Followup should include medical and psychological guidance, including continuing dietary advice and maintenance of a physical exercise regimen.

There is extensive knowledge to substantiate that exercise helps to gain a better physical fitness and Quality of Life (QoL). A Cochrane review showed a reduction of $1.5 \mathrm{~kg}$ that was contributed to exercise (7). Another systematic review, focussing on physical training and obesity, found a 4\% Excess Weight Loss (\%EWL) (8). In their review, Egberts et al. found a reduction of $3.6 \mathrm{~kg}$, related to physical exercise (9). In all three reviews, only prospective cohort studies were included as no randomized controlled could be identified. Weight measurement was merely self-reported and in the majority of studies the mode of physical activity was not defined. However, the positive relationship between exercise and weight loss seems evident. The problem is; what kind/type/mode of exercise should an obese patient be advised and what about it's timing, pre- or postoperative or a combination? These questions were the objective of the present review.

\subsection{METHODS}

A systematic search on the qualitative aspects and timing of physical exercise in bariatrics was conducted. The population of interest were all obese patients with a body mass index $(B M I) \geq 30 \mathrm{~kg} / \mathrm{m}^{2}$. The intervention of interest was exercise training compared to regular care (no specific physical exercise training program). Also included were the different modes of exercise training, those were compared to each other. Outcome measurements were; improvement of anthropometric and physical fitness variables; effect on weight and quality of life. 
Pubmed, Embase and CINAHL were searched from the earliest date of each database up to July 2014. The search for publications was performed using the following search string: ((“Bariatrics”[Mesh] OR bariatrics OR bariatric surgery OR “Obesity, Morbid”[Mesh] OR morbid obesity)) AND ("Physical Therapy Modalities”[Mesh] OR physical therapy OR physiotherapy OR "Exercise”[Mesh] OR exercise)

Authors MW and SP, blinded for authors and journals, separately screened and selected the studies on the basis of title and abstract. After primary selection, both authors reviewed the full text of the selected studies to determine suitability for inclusion, based on the established selection criteria.

- $\quad$ Randomised controlled trial or prospective trial.

- $\quad$ Patients awaiting bariatric surgical intervention or with bariatric surgery in the past.

- A description of an exercise program, defined as a regimen of physical activities included.

- Modes of exercise training were defined as follows: strength training and/or endurance training or a combination of both. Also multimodal programs with exercise components were included.

- Registration of anthropometric and physical fitness variables, complications, effects on weight and quality of life.

- Physical fitness variables are defined as a percentage of $\mathrm{VO}_{2}$ max and/or Heart rate reserve (HRR)/ Heart rate kinetics;

In addition cross-references were screened for further eligible studies. Disagreements between the two authors were resolved by discussion with each other and the senior authors (JT, $\mathrm{SN}$ ) until consensus was reached. It was conceived in advance as having significant clinical heterogeneity for which a systematic review could be performed, not a meta-analysis

The methodological quality of the included studies was rated using the Physiotherapy Evidence Database (PEDro) scale (10), which has acceptable reliability (11). The PEDro scale consists of 11 criteria, with a maximum score of 10 (range 0-10) since the first item (the specification of the eligibility criteria) is not included in the total score. Two authors (MW and SP) separately assigned Pedro scores to each included study. For rating the methodological quality the following classification was used: a PEDro score of 4 indicated a poor methodological quality, a score between 4-5 fair quality, score 6-8 good quality and 9-10 excellent quality (12). A Cohen's kappa score assessed the level of agreement between the authors. The score was determined as follows: $<0.20$ poor agreement; 0.21-0.40 fair agreement; 0.41-0.60 moderate agreement; 0.61-0.80 good agreement; 0.81-1.00 very good agreement (13). 


\subsection{RESULTS}

The primary search produced 1023 results, including 81 duplicates. Sixteen studies were identified as possibly relevant and underwent a full text critical appraisal, resulting in eight exclusions. One study investigated exercise programs for Prader-Willi patients (14), three studies investigated pulmonary physiotherapy in the bariatric population (15-17), one publication appeared to be a case-report (18), one a research protocol (19), one article was only available in Swedish (20) and one article was only available in Spanish (21).

Figure 8.1 summarises the search results. The methodological quality of the included studies ranged from moderate to good quality, as indicated by The PEDro scale (table 8.1). The level of agreement between reviewers (MW and SP) was reflected by a Cohen's kappa 0.78, which represents a good agreement. The key findings of the included studies are shown in tables 8.2 and 8.3 divided in pre-bariatric surgery exercise and post-bariatric surgery exercise.

\section{STUDY DESIGNS AND PATIENT POPULATION}

In total four pre-bariatric studies (22-25) and four post-bariatric studies (26-29) were included. Of the four pre-bariatric studies, the follow-up was only preoperatively, respectively 7 days (24), 12 weeks $(22,23), 24$ weeks $(25)$. Of the four post-bariatric studies, one of them had a postoperative follow-up of 12 weeks (26), three of them had a preoperative and postoperative follow-up (respectively 1 week preoperative and 4 weeks postoperative (27), preoperative (unknown how long) and 4 months postoperative (28) and 1 week preoperative and 10 days postoperative (29)). A total of three randomised controlled trials $(23,26,27)$ and five prospective trials $(22,24,25,28,29)$ studying a patient population with age ranging from 28 to 54 years old and a mean body mass index ranging from 40.4 to $48.7 \mathrm{~kg} / \mathrm{m}^{2}$. 


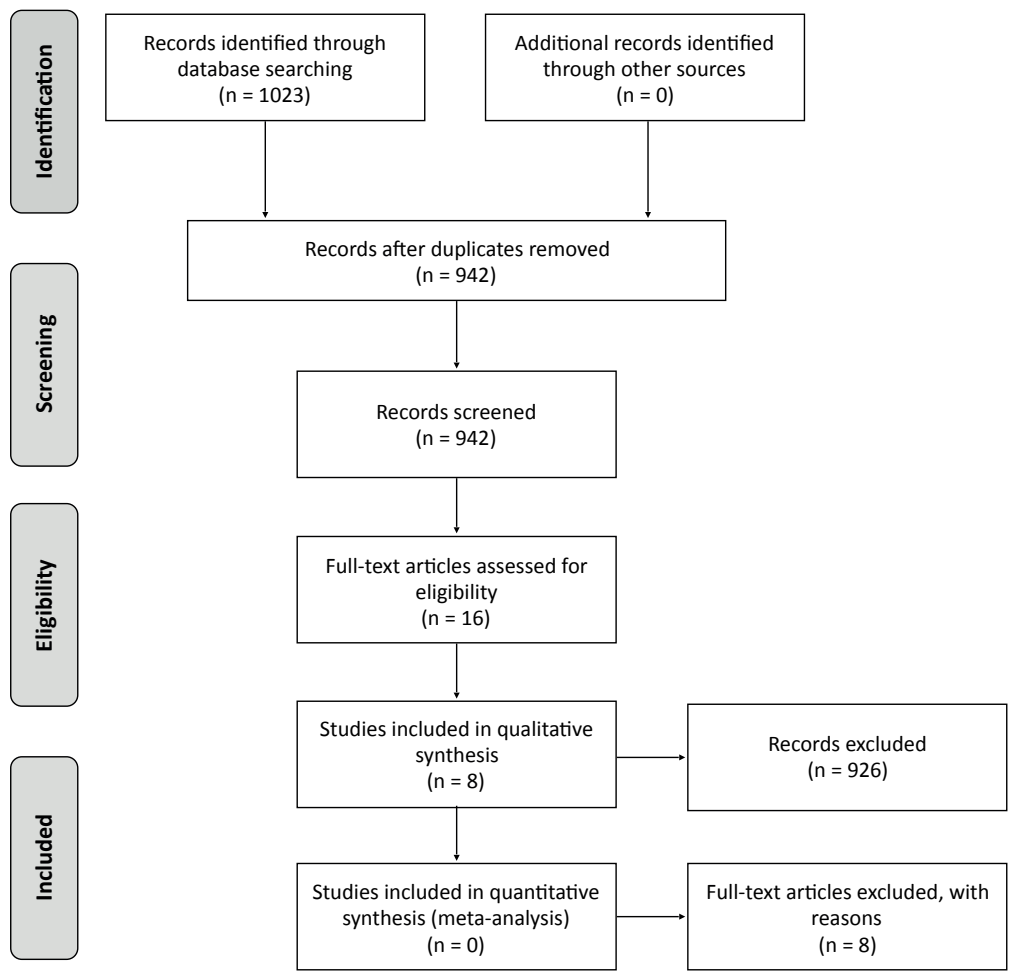

FIGURE 8.1. PRISMA Flowchart

TABLE 8.1. Methodological quality of included studies using the 'Physiotherapy Evidence Database (PEDro)' score (10)

\begin{tabular}{|c|c|c|c|c|c|c|c|c|c|c|c|c|}
\hline & \multicolumn{12}{|c|}{ Criteria $^{a}$} \\
\hline & 1 & 2 & 3 & 4 & 5 & 6 & 7 & 8 & 9 & 10 & 11 & Total \\
\hline Castello-Simoes et al. 2013 (27) & $\mathrm{x}$ & $\mathrm{x}$ & $\mathrm{x}$ & $\mathrm{x}$ & - & - & - & $\mathrm{x}$ & $\mathrm{x}$ & $\mathrm{x}$ & $\mathrm{x}$ & 7 \\
\hline Funderburk et al. 2010 (23) & $\mathrm{x}$ & $\mathrm{x}$ & $x$ & - & - & - & - & $\mathrm{x}$ & $\mathrm{x}$ & $\mathrm{x}$ & $\mathrm{x}$ & 6 \\
\hline Hickey et al. 1999 (24) & $\mathrm{x}$ & - & - & $\mathrm{x}$ & - & - & - & $\mathrm{x}$ & $\mathrm{x}$ & $\mathrm{x}$ & $\mathrm{x}$ & 5 \\
\hline Stegen et al. 2011 (28) & $\mathrm{x}$ & - & - & $\mathrm{x}$ & - & - & - & $\mathrm{x}$ & $\mathrm{x}$ & $\mathrm{x}$ & $\mathrm{x}$ & 5 \\
\hline Baillot et al. 2013 (22) & $\mathrm{x}$ & - & - & $\mathrm{x}$ & - & - & - & $\mathrm{x}$ & $\mathrm{x}$ & $\mathrm{x}$ & $\mathrm{x}$ & 5 \\
\hline Shah et al. 2011 (26) & $\mathrm{x}$ & $\mathrm{x}$ & $x$ & $\mathrm{x}$ & - & - & - & $\mathrm{x}$ & $\mathrm{x}$ & $\mathrm{x}$ & $\mathrm{x}$ & 7 \\
\hline Marcon et al. 2011 (25) & $\mathrm{x}$ & - & - & $\mathrm{x}$ & - & - & - & - & $\mathrm{x}$ & $\mathrm{x}$ & $\mathrm{x}$ & 4 \\
\hline Berggren et al. 2008 (29) & $\mathrm{x}$ & - & - & $\mathrm{x}$ & - & - & - & - & $\mathrm{x}$ & $\mathrm{x}$ & $\mathrm{x}$ & 4 \\
\hline
\end{tabular}

aPEDro criteria: (1) Specification of eligibility criteria (this criterion was not counted for the final score), (2) Random allocation, (3) Concealed allocation, (4) Prognostic similarity at baseline, (5) Participant blinding, (6) Therapist blinding, (7) Outcome assessor blinding, (8) More than 85\% follow-up of at least one key outcome, (9) Intention to treat analysis, (10) Between or within-group statistical comparison, (11) Point estimates of variability provided (10). 


\subsubsection{PRE-BARIATRIC SURGERY EXERCISE}

\section{Anthropometric variables}

Four studies reported on the effects of exercise training on the anthropometric variables. Baillot et al. (22) reported a significant reduction in weight (before; $144.3 \mathrm{~kg}$, after; 140.2 , $\mathrm{p}=0.07$ ), BMI (before; $51.4 \mathrm{~kg} / \mathrm{m}^{2}$, after; $47.2 \mathrm{~kg} / \mathrm{m}^{2}, \mathrm{p}=0.004$ ), neck circumference (before; $42.2 \mathrm{~cm}$, after; $41.0 \mathrm{~cm}, \mathrm{p}=0.016$ ) and fat mass (before; $72.1 \mathrm{~kg}$, after; $69.1 \mathrm{~kg}, \mathrm{p}=0.026$ ) after supervised exercise training in 12 patients awaiting bariatric surgery.

Funderburk et al. (23) showed a reduction in body weight after 12 weeks supervised aquatic exercises of $5.0 \mathrm{~kg}$ in the intervention group and $2.3 \mathrm{~kg}$ in the control group. It was not reported whether this is a significant decrease. There was no significant change in body weight and free fat mass after a seven day exercise regimen investigated by Hickey et al. (24) Marcon et al. (25) found a significant decrease in body weight $(-5.3 \mathrm{~kg}(\mathrm{p}<0.001))$ and BMI $(-1.9 \mathrm{~kg} / \mathrm{m} 2(\mathrm{p}<0.001))$ after 24 weeks supervised low intensity endurance training.

\section{Compliance}

Baillot et al. (22) had a high compliance to the preoperative exercise training program. They proposed 35 supervised exercise sessions in 12 weeks. Seven patients completed the full program and five had their bariatric surgery before the end of the program. The attendance of the supervised exercise sessions had a median of $57.3 \%(32.5-77.6 \%)$ and the attendance of the total exercise sessions had a median of $64.5 \%(47.5 \%-79.9 \%)$.

\section{Blood pressure}

After 12 weeks of aquatic exercises, Funderburk et al. (23) found an increase in systolic blood pressure and decrease in diastolic blood pressure in the intervention group $(+34 \mathrm{mmHg}$ and $-4 \mathrm{mmHg}$ respectively). In the control group, there was a decrease in systolic blood pressure and the diastolic blood pressure remained unchanged $(-57 \mathrm{mmHg}$ and $0 \mathrm{mmHg}$ ). (23) After 24 weeks of supervised endurance training Marcon et al (25) reported a significant decrease in systolic and diastolic blood pressure $(-23.8 \mathrm{mmHg}(\mathrm{p}=0.007)$ and $-14.4 \mathrm{mmHg}(\mathrm{p}=0.001)$ respectively).

\section{Cardiovascular risk factors and inflammation}

Hickey et al (24) reported a significant decrease of fasting plasma insulin (-41.7 pM) after seven days of supervised exercise training, 60 minutes per day. There were no significant changes in glucose and blood lipid concentration after the exercise intervention. (24) 
After 24 weeks of supervised endurance training a significant improvement was seen in blood lipid and glucose concentration in the study by Marcon et al. (25) (Total cholesterol: - 23.0 mg/dL; HDL-C: +1.7 mg/dL, LDL-C: -2.3 mg/dL, Triglycerides: -26.7 mg/dL, Glucose: -17.2 $\mathrm{mg} / \mathrm{dL}($ for all $\mathrm{p}<0.007)$ ).

\section{Physical fitness and functional capacity}

Baillot et al. (22) found that all twelve patients completed the baseline symptom limited treadmill test with a median duration of 11.5 minutes $(11.1$ - 12.4). No major cardiac complication was noted during or after the test. The six-minute walk test distance, the time of the half-squat test and the number of flexion during the arm curl test were all significantly $(\mathrm{p}<0.05)$ increased after the preoperative exercise program (22).

Funderburk et al. (23) showed an increase in the six-minute walking test distance of $10.4 \mathrm{~m}$, after 12 weeks of aquatic exercises, but strangely the control group showed an increase of 40.2 m. After 24 weeks Marcon et al. (25) also saw an improvement of six minute walking test distance $(+69.8 \mathrm{~m}(\mathrm{p}<0.0001))$.

\section{Aerobic capacity}

Hickey and coworkers (24) reported that after seven days of supervised exercise there was no significant difference in maximal oxygen uptake (which was measured by $\mathrm{VO}_{2}$ peak) and indicates that there is no increase in physical fitness.

\section{Quality of Life and satisfaction}

Baillot et al. (22) showed that each of twelve patients who were in the exercise program, improved at least one level for endurance training and two levels for strength training. This increase appeared to be greater in the patients who trained longer. All patients were satisfied by the coaching and the advices given by the exercise professionals (22).

Also the total Health Related Quality Of Life (HRQOL) significantly improved after the exercise intervention ( $\mathrm{p}=0.012$ ). On the domains of emotions, social interaction and sexual life was also a significant improvement noted $(\mathrm{p}=0.002, \mathrm{p}=0.025$ and $\mathrm{p}=0.003$ respectively) (22). Unfortunately, no significant change was seen in symptoms $(\mathrm{p}=0.25)$ activity/mobility ( $\mathrm{p}=0.07)$ and personal hygiene/clothing $(\mathrm{p}=0.078)$ scores. $(22)$

Measured by the Short Form 36 (SF-36) questionnaire and the Beck Depression inventory, Funderburk et al. (23) found no significant post intervention difference between groups. In the aquatic exercise group there was a significant decrease in bodily pain and a significant decrease of the depression score after 12 weeks $(\mathrm{p}<0.05)$. In the control group was a significant increase in mental health $(\mathrm{p}<0.05)$. (23) 
TABLE 8.2. Pre-bariatric surgery exercise regimes

\begin{tabular}{|c|c|c|}
\hline Study & Exercise regime & Outcome \\
\hline $\begin{array}{l}\text { Baillot } \\
\text { et al. } 2013 \\
\text { (22) }\end{array}$ & $\begin{array}{l}\text { The physical exercise sessions consisted of: } \\
10 \text { min of warm up, } \\
30 \text { min of endurance activity (treadmill, walking circuit), } \\
20 \text { to } 30 \text { min of strength exercises, } \\
10 \text { min of cooling-down. } \\
\text { Supervision by a physical activity specialist and/or } \\
\text { completed } \\
\text { independently at home. } \\
\text { The intensity of the endurance training was determined } \\
\text { using the HRR method and using data from the previously } \\
\text { performed symptom limited cardiac treadmill test. The } \\
\text { progression of endurance intensity and duration according } \\
\text { to physical fitness and physical activity. Electronic pulse } \\
\text { monitors (Polar F4】) were used to control endurance } \\
\text { exercise intensity during supervised sessions and at home. } \\
\text { Strength exercises: } \\
\text { Performed with small equipment easily available at home: } \\
\text { dumbbells, elastic bands, and sticks. Three mini-circuits } \\
\text { (upper body, lower body, and trunk) repeated by subjects } \\
\text { according to their prescription. }\end{array}$ & $\begin{array}{l}6 M W T \text { distance }(m): \\
\text { Before: } 464(412-501) \\
\text { After: } 492(450-531) \\
(\mathrm{p}=0.05) \\
\text { Arm Curl Test }(n): \\
\text { Before: } 22(16.7-26) \\
\text { After: } 26(21-31) \\
\text { (p=0.01) }\end{array}$ \\
\hline $\begin{array}{l}\text { Funderburk } \\
\text { et al. } 2010 \\
\text { (23) }\end{array}$ & $\begin{array}{l}\text { The intervention group did two sessions of } 60 \text { minutes } \\
\text { of aquatic exercises including endurance and strength } \\
\text { exercises for } 12 \text { weeks }\end{array}$ & $\begin{array}{l}\text { Weight loss: } \\
\text { I: }-5.0 \mathrm{~kg} \\
\mathrm{C}:-2.3 \mathrm{~kg} \\
\text { SBP and DBP: } \\
\mathrm{I}:+34 \mathrm{mmHg} \text { and }-4 \mathrm{mmHg} \\
\mathrm{C}:-57 \mathrm{mmHg} \text { and } 0 \mathrm{mmHg} \\
6 M W T: \\
\mathrm{I}:+10.4 \mathrm{~m} \\
\mathrm{C}:+40.2 \mathrm{~m} \\
\text { QoL and depression score: } \\
\text { No significant post intervention } \\
\text { difference between groups } \\
\text { I: decrease in bodily pain and } \\
\text { depression score (p<0.05) } \\
\text { C: Increase in mental health } \\
\text { (p<0.05) }\end{array}$ \\
\hline $\begin{array}{l}\text { Hickey } \\
\text { et al. } 1999 \\
(24)\end{array}$ & $\begin{array}{l}\text { Seven days of supervised endurance training at } 60 \% \text { of } \mathrm{VO}_{2} \\
\text { peak. Each session was } 60 \text { minutes. }\end{array}$ & $\begin{array}{l}\text { Decrease of fasting plasma insulin } \\
(-41.7 \mathrm{pM})(\mathrm{p}<0.05) \\
\text { No significant change in body } \\
\text { weight, \% fat mass, glucose and } \\
\text { blood lipid concentration after } \\
\text { intervention } \\
\text { No significant change in } \mathrm{VO}_{2} \text { peak } \\
\text { after exercise intervention }\end{array}$ \\
\hline
\end{tabular}




\begin{tabular}{|c|c|c|}
\hline $\begin{array}{l}\text { Marcon } \\
\text { et al. } 2011 \\
(25)\end{array}$ & $\begin{array}{l}24 \text { weeks of supervised low intensity endurance training. } \\
\text { One session per week, consisting of } 209 \text { minutes of } \\
\text { exercise and } 10 \text { minutes stretching. }\end{array}$ & $\begin{array}{l}\text { Body weight and BMI } \\
\text { Decreased body weight }-5.3 \mathrm{~kg} \\
\text { ( }<<0.001) \\
\text { Decreased BMI } \\
-1.9 \mathrm{~kg} / \mathrm{m}^{2}(\mathrm{p}<0.001) \\
\text { SBP and DBP: } \\
\text { Decrease in SBP and DBP }(-23.8 \\
\mathrm{mmHg}(\mathrm{p}=0.007) ;-14.4 \mathrm{mmHg} \\
\text { ( } \mathrm{p}=0.001) \\
\text { Lipids and glucose: } \\
\text { Improvement of TC, HDL-C, } \\
\text { LDL-C, TG and glucose } \\
\text { TC: }-23.0 \mathrm{mg} / \mathrm{dL} \\
\text { HDL-C: }+1.7 \mathrm{mg} / \mathrm{dL} \\
\text { LDL-C: }-2.3 \mathrm{mg} / \mathrm{dL} \\
\text { TG: }-26.7 \mathrm{mg} / \mathrm{dL} \\
\text { Glucose: }-17.2 \mathrm{mg} / \mathrm{dL} \\
\text { For all } \mathrm{p}<0.007 \\
6 M W T: \\
- \text { Improved walking distance; } \\
+69.8 \mathrm{~m}(\mathrm{p}<0.0001)\end{array}$ \\
\hline
\end{tabular}

Abbreviations: HRR = Heart rate reserve, $\mathrm{I}=$ Intervention group, $\mathrm{C}=$ Control group, $6 \mathrm{MWT}=6$ minute walking test, $\mathrm{n}=$ number, $\mathrm{SBP}=$ Systolic blood pressure, $\mathrm{DBP}=$ Diastolic blood pressure, $\mathrm{VO}_{2}$ peak $=$ maximum oxygen consumption, $\mathrm{BMI}=$ Body mass index, $\mathrm{TC}=$ Total cholesterol, $\mathrm{TG}=$ Triglycerides

\subsubsection{POST-BARIATRIC SURGERY EXERCISE}

\section{Anthropometric variables}

Four studies reported on the effects of exercise training on the anthropometric variables. Stegen et al. (28) investigated the effect of an exercise program after gastric bypass surgery and found that both groups (gastric bypass + exercise training $(\mathrm{GB}+\mathrm{E})$ compared with gastric bypass only (GB)) had the same decrease 4 months postoperative in weight (GB $=-26.6 \pm 14.6$ $\mathrm{kg} ; \mathrm{GB}+\mathrm{E}=-22.7 \pm 5.7 \mathrm{~kg}), \mathrm{BMI}\left(\mathrm{GB}=-8.3 \pm 4.1 \mathrm{~kg} / \mathrm{m}^{2} ; \mathrm{GB}+\mathrm{E}=-8.1 \pm 2.5 \mathrm{~kg} / \mathrm{m}^{2}\right)$ and waist circumference $(\mathrm{GB}=-20.3 \pm 11.6 \mathrm{~cm} ; \mathrm{GB}+\mathrm{E}=-17.2 \pm 8.1 \mathrm{~cm})(28)$.

Castello-Simoes (27) investigated a post-bariatric training program and used three groups: a trained group (TG), a eutrophic group (EG) and a control group (CG). They found that there was a significant reduction in weight (TG: before $115.0 \pm 6.9 \mathrm{~kg}$; after: $92.0 \pm 5.1 \mathrm{~kg}$, CG: before $113.0 \pm 4.7 \mathrm{~kg}$, after: $89.0 \pm 3.4 \mathrm{~kg}$ ) and BMI (TG: before $45.5 \pm 1.7 \mathrm{~kg} / \mathrm{m}^{2}$; after: 36.5 $\pm 1.3 \mathrm{~kg} / \mathrm{m}^{2}$, CG: before $43.6 \pm 1 \mathrm{~kg} / \mathrm{m}^{2}$, after: $\left.34.2 \pm 1.1 \mathrm{~kg} / \mathrm{m}^{2}\right)$ in TG and CG four months after bariatric surgery compared to the preoperative values (27).

Berggren and colleagues (29) investigated the skeletal muscle lipid oxidation before and after 10 days of endurance orientated exercise training. They found a significant weight loss after 10 days of exercise $(\mathrm{p}<0.05)$ (29). Shah et al. (26) investigated a high volume exercise program 
in 20 patients and found that in both the exercise group and the control group there was a significant reduction in body weight and hip circumference after 12 weeks (compared to baseline values).

\section{Cardiovascular risk factors and inflammation}

In contrast to the above mentioned, Shah et al. (26) found no difference in cardiovascular risk factors after a high volume exercise training program compared to the control group.

\section{Physical fitness and functional capacity}

Castello-Simoes (27) reported a significant increase in the 6 minute walking test distance $(6 \mathrm{MWT})$ after exercise training and bariatric surgery (before: $470 \pm 23.9 \mathrm{~m}$; after: $515.0 \pm$ $14.0 \mathrm{~m})$. Shah et al. (26) found a significantly increased energy expenditure and time spent on exercise in high-volume exercise group per day.

\section{Aerobic capacity}

In a maximal bicycle ergometer test before bariatric surgery, Stegen et al. (28) found that both groups (GB (gastric bypass only) and GB+E (gastric bypass and postoperative exercise program) reached their ventilator anaerobic threshold (VAT) at the power (GB $=93 \pm 24$ $\mathrm{W} ; \mathrm{GB}+\mathrm{E}=90 \pm 24 \mathrm{~W})$ with an equal time of occurrence $(\mathrm{GB}=270 \pm 107 \mathrm{~s} ; \mathrm{GB}+\mathrm{E}=266 \pm$ $133 \mathrm{~s}$ ) (28). Gastric bypass surgery did not improve time of occurrence of VAT and power at VAT. When patients followed an exercise program (GB+E group) postoperatively, the time of occurrence of VAT was delayed ( $349 \pm 19$ s) (28). Also Stegen and colleagues registered the peak exercise parameters. In the preoperative phase, morbidly obese patients reached a peak oxygen uptake of $17.4 \pm 4.9 \mathrm{ml} / \mathrm{kg} / \mathrm{min}(\mathrm{GB})$ and $17.6 \pm 3.2 \mathrm{ml} / \mathrm{kg} / \mathrm{min}(\mathrm{GB}+\mathrm{E})$. Unfortunately, absolute peak oxygen uptake and power did not improve 4 months after an intensive exercise program, nor by bariatric surgery (28).

Castello-Simoes (27) reported a significant increase of the predicted forced vital capacity (before: $94.0 \pm 3.1$; after: $101.0 \pm 2.5$ ) in the trained group 4 months after bariatric surgery (27). In the same group there was a significant reduction of the dyspnoea score (before: $5.8 \pm$ 0.6 ; after $2.7 \pm 0.8) 4$ months after bariatric surgery.

\section{Muscle strength}

Stegen et al. (28) found that the untrained patients (who only had a gastric bypass (GB)) had a decrease in dynamic muscle strength 4 months postoperative. They have lost $16 \%$ of their quadriceps strength, 36\% of their biceps strength and 39\% of triceps strength. Patients who had a gastric bypass and followed an exercise program postoperative prevented this decrease. Biceps $($ Pre $=21.8 \pm 8.0 \mathrm{~kg}$; Post $=25.9 \pm 13.0 \mathrm{~kg})$ and triceps $($ Pre $=24.3 \pm 10.1 \mathrm{~kg} ;$ Post $=30.7$ 
$\pm 23.0 \mathrm{~kg}$ ) strength were preserved and both hamstrings and quadriceps strength increased ( $72 \%$ and $27 \%$ respectively) due to the exercise program (28). Static muscle strength, which is measured as handgrip strength, decreased in both groups 4 months after gastric bypass surgery with $18 \%$ and $7 \%$ respectively. Muscle fatigue was not influenced by gastric bypass surgery, nor by an additional exercise training program (28).

\section{Fatty acid oxidation}

Berggren et al. (29) studied the effect of weight loss through exercise training on the muscle fatty acid oxidation. Muscle fatty acid oxidation in extremely obese women who had lost weight (approximately $50 \mathrm{~kg}$ ) was compared with extremely obese and lean individuals (29). There was no difference in muscle fatty acid oxidation between extremely obese and the weight loss groups. The fatty acid oxidation was depressed compared with the lean individuals $(-45 \%$; $\mathrm{P}<0.05$ ). In contrast, ten days of exercise training increased fatty acid oxidation in the skeletal muscle of lean, obese and previously extreme obese subjects after weight loss (respectively + 1.7 -fold, +1.8 -fold and +2.6 -fold) (29).

\section{Quality of Life and satisfaction}

Shah et al (26) reported in the high volume exercise group (HVEP) a significant improvement in health related quality of life on several domains including physical function $(p=0.049)$, self-esteem $(\mathrm{p}=0.0002)$, sexual life $(\mathrm{p}=0.02)$, public distress $(\mathrm{p}=0.003)$, and the total score $(\mathrm{p}=0.0004)$ over 12 weeks. The control group showed a significant improvement in selfesteem ( $\mathrm{p}=0.004)$, sexual life $(\mathrm{p}=0.04)$, and work (or daily activities) $(\mathrm{p}=0.04)$ and the total score $(\mathrm{p}=0.012)$. (26) There was no group-by-week interaction for any of the scales except the interaction for self-esteem, which was approximately $p=0.05$. The data from the SF-36 questionnaire showed that there was no group-by-week interaction for any of the scales, but the HVEP group reported a significant improvement in emotional well being $(p=0.001)$, energy levels ( $\mathrm{p}=0.0002)$, and mental QOL total score $(\mathrm{p}=0.006)$ over 12 weeks whereas QOL in the control group did not change. (26) 
TABLE 8.3. Post- bariatric surgery exercise regimes

\begin{tabular}{|c|c|c|}
\hline Study & Exercise regime & Outcome \\
\hline $\begin{array}{l}\text { Shah } \\
\text { et al. } 2011 \\
(26)\end{array}$ & $\begin{array}{l}\text { Energy expenditure } \geq 2,000 \mathrm{kcal} / \text { week in moderate-intensity aerobic } \\
\text { exercise at } 60-70 \% \text { of maximal oxygen consumption (VO2max). } \\
\text { The subjects were instructed to achieve these goals gradually and were } \\
\text { asked to expend } 500 \mathrm{kcal} \text { during the first week and increase by } 500 \mathrm{kcal} \\
\text { every week until they achieved their goal of } \geq 2,000 \mathrm{kcal} / \text { week. } \\
\text { Each subject was asked to exercise on the treadmill at a certain } \\
\text { speed and grade and on the cycle ergometer or rowing machine at } \\
\text { a particular wattage that would correspond to } 60-70 \% \text { of her/his } \\
\text { measured VO2max. } \\
\text { Subjects who preferred to walk outside or on the walking track were } \\
\text { asked to measure the distance that they walked and asked to complete } \\
\text { this distance in a time period to achieve a pace associated with an } \\
\text { intensity of } 60-70 \% \text { of the measured VO2max. } \\
\text { The subjects were asked to exercise at least } 5 \text { days a week and to } \\
\text { increase the intensity of exercise to remain the same level of exertion } \\
\text { after each training. } \\
\text { The exercise was partially supervised and the subjects were asked to } \\
\text { come to the fitness center at least once or twice a week. } \\
\text { Energy expenditure per week was calculated approximately from the } \\
\text { work data shown on the exercise equipment and from the duration } \\
\text { and distance of walking relative to body weight. } \\
\text { Exercise away from the fitness center was monitored by asking the } \\
\text { subjects to keep an exercise diary and/or using heart rate monitors. }\end{array}$ & $\begin{array}{l}\text { Energy expenditure } \\
\text { (moderate physical } \\
\text { activity) }(\mathrm{kcal} / \mathrm{kg} / \\
\text { day): } \\
\text { I: Baseline } 1.1 \\
(0-5.8) \text {, } \\
6 \text { weeks } 3.5(0.4- \\
8.2)(\mathrm{p}<0.02) \text {, } \\
12 \text { weeks } 4.1(0- \\
15.0)(\mathrm{p}=<0.0001) \\
\text { Time spent } \\
\text { (moderate physical } \\
\text { activity) }(\mathrm{h} / \text { day): } \\
\mathrm{I}: \text { Baseline } 0.3 \\
(0-1.5), \\
6 \text { weeks } 0.9(0.1- \\
2.1)(\mathrm{p}<0.02) \\
12 \text { weeks } 1.0(0- \\
3.8)(\mathrm{p}<0.0001)\end{array}$ \\
\hline
\end{tabular}




\begin{tabular}{|c|c|c|}
\hline $\begin{array}{l}\text { Castello-Simoes } \\
\text { et al. } 2013 \\
\text { (27) }\end{array}$ & $\begin{array}{l}\text { Start of aerobic training } 48 \text { hours after maximal exercise test. } \\
\text { Session duration } 1 \text { hour on alternate days, } 3 \text { times a week, for } 12 \\
\text { weeks, totaling } 36 \text { sessions. } \\
\text { A session consisted of the following: } \\
\text { initial } 5 \text { min stretching of upper and lower limbs and diaphragmatic } \\
\text { breathing. } \\
5 \text { min warm up on a treadmill at } 3 \mathrm{~km} / \mathrm{h} \text {. } \\
40 \text { min of exercise on a treadmill with speed and inclination varying } \\
\text { according to the behaviour of HR, separated in } 4 \text { steps; } \\
\text { Step } 1 \text { - intensity of exercise in which the HR remained at } 50 \% \text { of HR } \\
\text { peak, reached in maximal exercise testing. } \\
\text { Step } 2 \text { - } 60 \% \text { of HR peak. } \\
\text { Step } 3 \text { - } 70 \% \text { of HR peak. } \\
\text { Step } 4 \text { - Maintaining } 70 \% \text { of the HR peak. } \\
1 \text { min recovery at } 3 \mathrm{~km} / \mathrm{h} \text {. } \\
10 \text { min of the same initial stretching and diaphragmatic breathing. } \\
\text { HR and blood pressure were obtained at the beginning of the session, } \\
\text { at the end of each step, recovery and at the end of the session. }\end{array}$ & 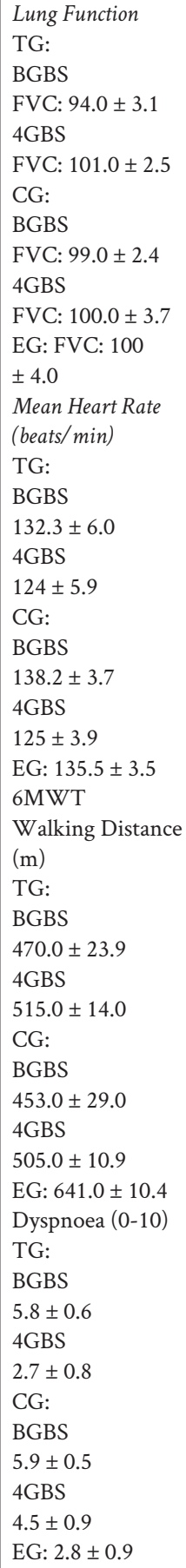 \\
\hline
\end{tabular}




\begin{tabular}{|c|c|c|}
\hline $\begin{array}{l}\text { Stegen } \\
\text { et al. } 2011 \\
(28)\end{array}$ & $\begin{array}{l}\text { Strength exercises were performed using stack-weight equipment (elbow } \\
\text { flexion and extension and knee flexion and extension). Intensity of } \\
\text { strength training was calculated from one repetition maximum (1RM) } \\
\text { values. } \\
\text { The starting level was } 60 \% \text { of } 1 \mathrm{RM} \text {, which increased up to } 75 \% \text { of } \\
\text { 1RM. Patients were habituated to the exercises and equipment during } \\
\text { the first three training sessions. } \\
\text { In the next } 15 \text { sessions, two sets of } 15 \text { repetitions at } 60 \% \text { of } 1 \mathrm{RM} \text { were } \\
\text { done. } \\
\text { In the final } 18 \text { sessions, three sets of } 10 \text { repetitions at } 75 \% \text { were done. } \\
\text { Resting periods between repetition sets were approximately } 60 \text { s. } \\
\text { Endurance training consisted of cycling ( } 10 \text { min), walking ( } 10 \text { min), and } \\
\text { stepping ( } 10 \text { min). Exercise intensity was initially set at } 60 \% \text { of heart } \\
\text { rate reserve (HRR, defined as the difference between resting heart rate } \\
\text { and peak heart rate) and was gradually increased up to } 75 \% \text { of HRR } \\
\text { at the end of the training period. Stationary bicycles, treadmills, and } \\
\text { steppers were used in a rehabilitation training room. }\end{array}$ & $\begin{array}{l}\text { Dynamic muscle } \\
\text { strength in } \\
\text { kg }(4 \text { months } \\
\text { postoperative): } \\
\text { Quadriceps: } \\
\text { I: } 58.0( \pm 25.6) \\
\text { C: } 45.9(25.1) \\
\text { (p=0.002) } \\
\text { Biceps } \\
\text { I: } 25.9( \pm 13.0) \\
\text { C: } 20.8( \pm 8.8) \\
(\mathrm{p}=0.001) \\
\text { Triceps } \\
\text { I: } 30.7( \pm 23.0) \\
\text { C: } 22.0( \pm 6.6) \\
(\mathrm{p}=0.038)\end{array}$ \\
\hline $\begin{array}{l}\text { Berggren } \\
\text { et al. } 2008 \\
(29)\end{array}$ & $\begin{array}{l}10 \text { consecutive days of endurance-oriented exercise training. } \\
\text { An incremental, maximal stress test was initially performed to screen } \\
\text { for underlying cardiovascular disease and determine the workload } \\
\text { [\%peak oxygen consumption and heart rate peak] for the exercise } \\
\text { training. } \\
\mathrm{VO}_{2} \text { peak was determined on an electronically braked cycle ergometer } \\
\text { in the upright position, with oxygen consumption measured via open- } \\
\text { circuit spirometry. } \\
\text { The training program was based upon previous exercise prescriptions } \\
\text { that improved whole body fat oxidation or increased mitochondrial } \\
\text { content. } \\
\text { Participants exercised } 60 \text { min/day at } 70 \% \mathrm{VO}_{2} \text { peak for } 10 \text { consecutive } \\
\text { days on a cycle ergometer. } \\
\text { All training was performed under direct supervision. } \\
\text { Subjects were initially permitted to take a rest break ( } 5 \text { min) during } \\
\text { exercise; by day } 5 \text { all subjects exercised continuously. }\end{array}$ & $\begin{array}{l}\text { Muscle fatty acid } \\
\text { oxidation: } \\
\text { Lean subjects: } \\
\text { increase }+1.7 \text {-fold } \\
\text { Obese subjects: } \\
\text { increase }+1.8 \text {-fold } \\
\text { Previously extreme } \\
\text { obese subjects } \\
\text { after weight loss: } \\
\text { increase }+2.6 \text {-fold }\end{array}$ \\
\hline
\end{tabular}

Abbreviations: GBS = Gastric Bypass, TG = Trained Group, CG = Control Group, EG = Eutrophic Group, $\mathrm{BGBS}=$ before gastric bypass surgery, $4 \mathrm{GBS}=4$ months after gastric bypass surgery, $\mathrm{W}=$ Weight, $\mathrm{BMI}=\mathrm{Body}$ Mass Index $\left(\mathrm{kg} / \mathrm{m}^{2}\right), 6 \mathrm{MWT}=6$ minute walking test, $\mathrm{FVC}=$ Forced Vital Capacity, $\mathrm{HR}=$ heart rate, $1 \mathrm{RM}=$ One repetition maximum. HRR = heart rate reserve, $\mathrm{I}=$ Intervention group, $\mathrm{C}=$ Control group 


\subsection{DISCUSSION}

Considering the great value assigned to exercise, a search for evidence provided a relatively little number of prospective studies addressing this subject in relation to bariatric surgery. In the majority of reports a positive effect on anthropometrics, cardiovascular risk factors and physical fitness was described. However, the results were not unanimous, with a wide range of exercise programs and peri-operative timing and therefore hampering adequate practical guidance. Simplifying the common findings an exercise program lasts for a median of 12 weeks with an intensity of a median $65 \%$ peak heart rate $/ \mathrm{VO}_{2} \max$ and at least partially supervised (table 8.4).

Two systematic reviews (by Baillot et al. (30) and Fonseca-Junior et al. (31)) assessed the impact of physical exercise and fitness in obese individuals, but without an in depth look on the timing peri-operatively and type of exercise needed to improve health in the bariatric population. The results of exercise in terms of weight loss and changes in the other anthropometric variables have been described before (1-3). However the type of exercise, used to achieve this weight loss, was not specified / given in detail. These details could be relevant as exercise regimes can include endurance training, strength training or combinations of intensity of both components. Unfortunately, it is difficult to assess the appropriate type and amount of physical activity for the bariatric surgical patient. It is unclear if preoperative exercise has the same effect as postoperative exercise. Also there is no uniformity on the outcome variables and little is known about the effect of exercise programs on the weight regain after bariatric surgical interventions. Nevertheless, it is known that exercise results in significant changes in anthropometric parameters (22-29) and results in more weight loss than bariatric surgery alone (7-9).

Exercise training demonstrates favourable effects on body composition, blood pressure control, insulin sensitivity, inflammation biomarkers and subclinical carotid atherosclerosis (CIMT). These effects have been associated with evidence of removal of oxidized phospholipids from the vessel wall, stabilisation of atherosclerosis and positive changes in LDL cholesterol (32). These changes have a strong correlation with increase in vascular function and regression in the burden of atherosclerosis (33). Ahmadi et al. (34) showed that the CIMT decrease was significantly greater in response to a 7 -month exercise program than statin or niacin therapies in the ARBITER 6 trial (35). In other words, exercise training reduces cardiovascular risk in obese individuals. 
TABLE 8.4. Common findings

\begin{tabular}{|c|c|c|c|c|c|c|c|c|}
\hline & 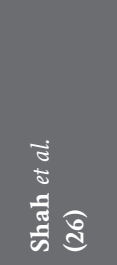 & 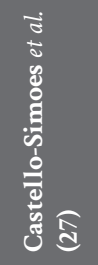 & 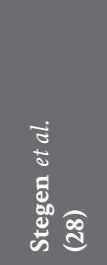 & 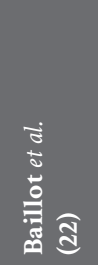 & 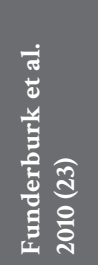 & 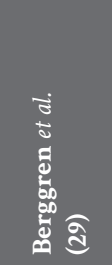 & 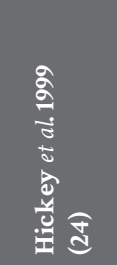 & 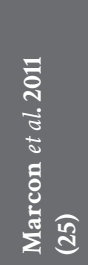 \\
\hline Duration (weeks) & 12 & 12 & 16 & 12 & 12 & 2 & 1 & 24 \\
\hline Intensity & $\begin{array}{c}60-70 \% \\
\mathrm{VO}_{2} \max \end{array}$ & $\begin{array}{c}60-70 \% \\
\text { HR }\end{array}$ & $\begin{array}{l}60-75 \% \\
\text { HR/RM }\end{array}$ & $\begin{array}{l}55-85 \% \\
\text { of HRR }\end{array}$ & $\mathrm{x}$ & $\begin{array}{c}70 \% \\
\mathrm{VO}_{2} \max \end{array}$ & $\begin{array}{c}65 \% \\
\mathrm{VO}_{2} \text { peak }\end{array}$ & $\mathrm{x}$ \\
\hline Supervision & Partial & Whole & Whole & Partial & Whole & $\mathrm{x}$ & Whole & Whole \\
\hline $\begin{array}{l}\text { Timing bariatric } \\
\text { surgery }\end{array}$ & Post & Post & Post & Pre & Pre & Post & Pre & Pre \\
\hline
\end{tabular}

Abbreviations: $\mathrm{HR}=$ Heart rate, $\mathrm{HRR}=$ Heart rate reserve, $\mathrm{RM}=$ Repeated Measurements, $2 \mathrm{KmWT}=2$ kilometre walking test, $\mathrm{x}=$ unknown

Bariatric surgery is the only longstanding intervention when conservative therapy fails to succeed. However bariatric surgery also has consequences. One of them is a decrease of dynamic muscle strength after the operation (28). A possible explanation could be the found in a decrease of muscle mass after the operation. In the study of Stegen et al. (28) the untrained patients lost a total of $7.6 \mathrm{~kg}$ of muscle mass. That is $29.7 \%$ of the total body weight lost $(-26.6$ $\mathrm{kg}$ ) through bariatric surgery.

Reviews by Stiegler et al. (36) and Chaston et al. (37) confirmed the positive correlation between weight loss and fat-free mass loss (FFML). Very low caloric diets result in a greater FFML compared to moderate caloric diets. Bariatric surgery results in greater FFML than very low caloric diets. Webster et al. (38) found that the FFML should not exceed the $22 \%$ of the total weight loss because of the function in resting metabolic rate, thermoregulation, oxidative capacity of the body and weight management. Exercise training can attenuate muscle atrophy and can maintain FFM during weight loss $(36,37)$, but the value of a perioperative exercise program for bariatric surgery has not been investigated.

It can be debated whether or not decrease of muscle strength is a problem for morbidly obese patients after weight loss. It is well known that obese individuals have higher absolute muscle strength compared to lean subjects, but there is lower relative muscle strength in terms of total body weight (39-42). This gives patients an impaired functional capacity, which results in the fact that more strength is needed to handle a heavier body. Therefore it might be important to prevent a decrease in muscle strength after bariatric surgery. However when investigating muscle changes before and after bariatric surgery, several factors have to be 
taken into account. One of them is the type of bariatric surgery. In the study by Stegen and coworkers (28) only patients who have undergone gastric bypass surgery were included. To our knowledge it is unknown whether there are differences in muscle loss between different types of bariatric surgery (per example gastric bypass surgery compared to a sleeve gastrectomy). Secondly, the patient compliance must also be studied, e.i. if patients followedup the required dietary instructions ((re)substitution of proteins, vitamins and minerals). (3638) None of these data were measured in the included studies.

It is known that obese patients have a reduced functional capacity and walking distance compared to lean subjects (43). A study of Tompkins and colleagues (44) showed that bariatric surgery leads to an improvement of approximately $75 \%$ in functional capacity and walking capacity 6 months after the operation. Weight loss (especially loss of body fat) makes walking less exhausting which was noticed by a decreased rate of exertion and heart frequency at the end of the 6 minute walking test in the studies of Stegen et al. (28) and Castello-Simoes et al. (27). Important predictors are not only the percentage of body fat, but also the maximum of oxygen uptake $\left(\mathrm{VO}_{2} \max \right)$ and the quadriceps muscle strength $(43,45,46)$.

Exercise capacity can be measured by the anaerobic threshold (AT), which reflects the true aerobic capacity and by the maximal oxygen uptake $\left(\mathrm{VO}_{2}\right.$ peak). To interpret these parameters we must realise there are differences between obese and lean subjects. Healthy obese subjects undergoing weight loss will experience a decrease in the maximal oxygen uptake and peak work output, because of a decrease of muscle mass (47), and a elevated cardiac stress (which is training for the heart). This cardiac stress will fade away as matter of time (48).

In contrast, many obese patients suffer from obesity-related disorders, such as impaired cardiac function $(49,50)$, inefficient ventilatory work (51) and a decreased fatty acid oxidation $(29,52)$. These disorders are associated with a reduced exercise capacity. During weight loss, both the cardiac and pulmonary systems will restore and may overwhelm the decrease in muscle mass. This delicate balance might result in a stabilisation or even an improvement in maximal oxygen uptake.

Our study has several limitations that have to be taken into account. First, weight loss measured in the included studies was merely self-reported. In ideal circumstances, exercise programs and the effects of them must be monitored frequently (and objectively) by visiting a bariatric clinic. Second, because of the small sample sizes of the included studies and the heterogeneity in reported exercise programs and outcomes, it is difficult to interpret and generalise the findings for the whole bariatric population. 
It is well known that exercise programs (with or without bariatric surgery) give a decrease in anthropometric parameters, but there are more beneficial effects of exercise. For future research there must be a focus to compare different exercise regimes (endurance and strength per example) in obese subjects. There is also an increasing need for randomised controlled trials that focus on the effect of peri-bariatric exercise on weight regain after bariatric surgery, because this is still an ongoing problem. Studies by Bond and colleagues (53) and Li and colleagues (54) investigate whether a preoperative exercise program has effects on the postoperative outcome. The definitive results of those studies are not published yet. Also the long-term effects of exercise programs on quality of life, depressive and anxiety symptoms and eating behaviour are not well understood.

\subsection{CONCLUSION}

In the majority of reports on exercising in a (future) bariatric population, a positive effect on anthropometrics, cardiovascular risk factors and physical fitness was described. However, the results were not unanimous, with a wide range of exercise programs and peri-operative timing and therefore hampering adequate practical guidance. Summarising our findings, a beneficial exercise program lasts for a median of 12 weeks with an intensity of a median $65 \%$ peak heart rate $/ \mathrm{VO}_{2} \max$ and at least partially supervised. 


\subsection{REFERENCES}

1. Obesity: preventing and managing the global epidemic. Report of a WHO consultation. World Health Organization technical report series. 894:i-xii, 1-253 (2000).

2. M. Tjepkema. Adult obesity. Health reports. 17:9-25 (2006).

3. R. Sturm. Increases in morbid obesity in the USA: 2000-2005. Public health. 121:492-496 (2007).

4. J. Picot, J. Jones, J.L. Colquitt, E. Gospodarevskaya, E. Loveman, L. Baxter, and A.J. Clegg. The clinical effectiveness and cost-effectiveness of bariatric (weight loss) surgery for obesity: a systematic review and economic evaluation. Health technology assessment (Winchester, England). 13:1-190, 215-357, iii-iv (2009).

5. J.L. Colquitt, J. Picot, E. Loveman, and A.J. Clegg. Surgery for obesity. The Cochrane database of systematic reviews:CD003641 (2009).

6. H. Buchwald. The evolution of metabolic/bariatric surgery. Obes Surg. 24:1126-1135 (2014).

7. K. Shaw, H. Gennat, P. O'Rourke, and C. Del Mar. Exercise for overweight or obesity. The Cochrane database of systematic reviews:CD003817 (2006).

8. M. Livhits, C. Mercado, I. Yermilov, J.A. Parikh, E. Dutson, A. Mehran, C.Y. Ko, and M.M. Gibbons. Exercise following bariatric surgery: Systematic review. Obes Surg. 20:657-665 (2010).

9. K. Egberts, W.A. Brown, L. Brennan, and P.E. O’Brien. Does exercise improve weight loss after bariatric surgery? A systematic review. Obes Surg. 22:335-341 (2012).

10. A.M. Mosely, R.D. Herbert, and C. Sherrington. Evidence for physiotherapy practice: a survey of the Physiotherapy Evidence Database (PEDro). Aust J Physiother. 48:43-49 (2002).

11. C.G. Maher, C. Sherrington, R.D. Herbert, and e. al. Reliability of the PEDro scale for rating quality of randomized controlled trials. Phys Ther. 83:713-721 (2003).

12. N.C. Foley, R.W. Teasell, S.K. Bhogal, and e. al. Stroke rehabilitation evidence-based review: methodology. Top Stroke Rehabil. 10:1-7 (2003).

13. D. Altman. Practical statistics for medical research., Chapman and Hal, London, 1991.

14. L. Vismara, V. Cimolin, G. Grugni, M. Galli, C. Parisio, O. Sibilia, and P. Capodaglio. Effectiveness of a 6-month home-based training program in Prader-Willi patients. Research in developmental disabilities. 31:1373-1379 (2010).

15. M.C. Barbalho-Moulim, G.P. Miguel, E.M. Forti, A. Campos Fdo, and D. Costa. Effects of preoperative inspiratory muscle training in obese women undergoing open bariatric surgery: respiratory muscle strength, lung volumes, and diaphragmatic excursion. Clinics (Sao Paulo, Brazil). 66:1721-1727 (2011).

16. V. Bernhardt, S. Lorenzo, T.G. Babb, and G.S. Zavorsky. Corrected end-tidal $\mathrm{P}(\mathrm{CO}(2))$ accurately estimates $\mathrm{Pa}(\mathrm{CO}(2))$ at rest and during exercise in morbidly obese adults. Chest. 143:471-477 (2013).

17. D. Cattano, A. Altamirano, V. Melnikov, E. Pivalizza, A. Feldman, and C. Hagberg. Postoperative pulmonary inspiratory reserve volume and incentive spirometry in morbidly obese patients undergoing bariatric surgery. Journal of Investigative Medicine. 58 (4):678-679 (2010).

18. R. Marzocchi, D. Cappellari, R. Dalle Grave, and G. Marchesini. Massive weight loss without surgery in a super obese patient. Obes Surg. 21:540-545 (2011). 
19. A.G. Zapico, P.J. Benito, M. Gonzalez-Gross, A.B. Peinado, E. Morencos, B. Romero, M.A. RojoTirado, R. Cupeiro, B. Szendrei, J. Butragueno, M. Bermejo, M. Alvarez-Sanchez, M. GarciaFuentes, C. Gomez-Candela, L.M. Bermejo, C. Fernandez-Fernandez, and F.J. Calderon. Nutrition and physical activity programs for obesity treatment (PRONAF study): methodological approach of the project. BMC public health. 12:1100 (2012).

20. G. Adolfsson. Physical training and weight reduction in connection with operations. [Swedish] Fysisk traning och viktreduktion vid operation. Lakartidningen. 67:4941-4944 (1970).

21. L. Sanchez Ortega, C. Sanchez Juan, and A.A. Garcia. Evaluation of a structured program of physical exercise in morbidly obese patients awaiting bariatric surgery. Nutricion hospitalaria. 29:64-72 (2014).

22. A. Baillot, W. Mampuya, E. Comeau, A. Meziat-Burdin, and M. Langlois. Feasibility and impacts of supervised exercise training in subjects with obesity awaiting bariatric surgery: a pilot study. Obes Surg. 23:882-891 (2013).

23. J.A. Funderburkand S. Callis. Aquatic Intervention effect on quality of life prior to obesity surgery: a pilot study. Annu in Ther Recreation. 18:66-78 (2010).

24. M.S. Hickey, K.E. Gavigan, and M.R. McGammon. Effects of 7 days of exercise training on insulin action in morbidly obese men. Clin Exerc Physiol. 1:24-28 (1999).

25. E.R. Marcon, I. Gus, and C.R. Neumann. Impact of a minimum program of supervised exercises in the cardiometabolic risk in patients with morbid obesity. Arq Bras Endocrinol Metabol. 55:331338 (2011).

26. M. Shah, P.G. Snell, S. Rao, B. Adams-Huet, C. Quittner, E.H. Livingston, and A. Garg. Highvolume exercise program in obese bariatric surgery patients: a randomized, controlled trial. Obesity (Silver Spring, Md). 19:1826-1834 (2011).

27. V. Castello-Simoes, R. Polaquini Simoes, T. Beltrame, D. Bassi, A. Maria Catai, R. Arena, N.C. Azambuja, Jr., J. do Nascimento Ortega, and A. Borghi-Silva. Effects of aerobic exercise training on variability and heart rate kinetic during submaximal exercise after gastric bypass surgery--a randomized controlled trial. Disability and rehabilitation. 35:334-342 (2013).

28. S. Stegen, W. Derave, P. Calders, C. Van Laethem, and P. Pattyn. Physical fitness in morbidly obese patients: effect of gastric bypass surgery and exercise training. Obes Surg. 21:61-70 (2011).

29. J.R. Berggren, K.E. Boyle, W.H. Chapman, and J.A. Houmard. Skeletal muscle lipid oxidation and obesity: influence of weight loss and exercise. American journal of physiology Endocrinology and metabolism. 294:E726-732 (2008).

30. A. Baillot, M. Audet, J.P. Baillargeon, I.J. Dionne, L. Valiquette, M.M. Rosa-Fortin, C.N. Abou Chakra, E. Comeau, and M.F. Langlois. Impact of physical activity and fitness in class II and III obese individuals: a systematic review. Obes Rev (2014).

31. S.J. Fonseca-Junior, C.G. Sa, P.A. Rodrigues, A.J. Oliveira, and J. Fernandes-Filho. Physical exercise and morbid obesity: a systematic review. Arquivos brasileiros de cirurgia digestiva : $\mathrm{ABCD}=$ Brazilian archives of digestive surgery. 26 Suppl 1:67-73 (2013).

32. C. Bergmark, A. Dewan, and A. Orsoni. A novel function of lipoprotein [a] as a preferential carrier of oxidized phospholipids in human plasma. J Lipid Res. 49:2230-2239 (2008). 
33. N. Ahmadi, S. Tsimikas, and F. Hajsadeghi. Relation of oxidative biomarkers, vascular dysfunction, and progression of coronary artery calcium. Am J Cardiol. 105:459-466 (2010).

34. N. Ahmadi, S. Eshaghian, R. Huizenga, K. Sosnin, R. Ebrahimi, and R. Siegel. Effects of intense exercise and moderate caloric restriction on cardiovascular risk factors and inflammation. The American journal of medicine. 124:978-982 (2011).

35. A.J. Taylor, T.C. Villines, and E.J. Stanek. Extended-release niacin or ezetimibe and carotid intima-media thickness. N Engl J Med. 361:2113-2122 (2009).

36. P. Stieglerand A. Cunliffe. The role of diet and exercise for the maintenance of fat-free mass and resting metabolic rate during weight loss Sports Med. 36:239-262 (2006).

37. T.B. Chaston, J.B. Dixon, and P.E. O’Brien. Changes in fat-free mass during significant weight loss: a systematic review. Int J Obes (Lond). 31:743-750 (2007).

38. J.D. Webster, R. Hesp, and J.S. Garrow. The composition of excess weight in obese women estimated by body density, total body water and total body potassium. Hum Nutr Clin Nutr. 38:299-306 (1984).

39. N.A. Maffiuletti, M. Jubeau, and U. Munzinger. Differences in quadriceps muscle strength and fatigue between lean and obese subjects. Eur J Appl Physiol. 101:51-59 (2007).

40. C.J. Blimkie, D.G. Sale, and O. Bar-Or. Voluntary strength, evoked twitch contractile properties and motor unit activation of knee extensors in obese and non-obese adolescent males. Eur J Appl Physiol Occup Physiol. 61:313-318 (1990).

41. L.S. Pescatello, B.K. Kelsey, and T.B. Price. The muscle strength and size response to upper arm, unilateral resistance training among adults who are overweight and obese. J Strength Cond Res. 21:307-313 (2007).

42. M. Hulens, G. Vansant, and R. Lysens. Study of differences in peripheral muscle strength of lean versus obesewomen: an allometric approach. Int J Obes Relat Metab Disord. 25:676-681 (2001).

43. M. Hulens, G. Vansant, and A.L. Claessens. Predictors of 6-minute walk test results in lean, obese and morbidly obese women. Scand J Med Sci Sports. 13:98-105 (2003).

44. J. Tompkins, P.R. Bosch, and R. Chenowith. Changes in functional walking distance and healthrelated quality of life after gastric bypass surgery. Phys Ther. 88:928-935 (2008).

45. R. Laukkanen, P. Oja, and M. Pasanen. Validity of a two kilometre walking test for estimating maximal aerobic power in overweight adults. Int J Obes Relat Metab Disord. 16:263-268 (1992).

46. E. Mattsson, U.E. Larsson, and S. Rossner. Is walking for exercise too exhausting for obese women? Int J Obes Relat Metab Disord. 21:380-386 (1997).

47. M. Goran, D.A. Fields, and G.R. Hunter. Total body fat does not influence maximal aerobic capacity. 2000. 24:841-848 (2000).

48. L. Seres, J. Lopez-Ayerbe, and R. Coll. Increased exercise capacity after surgically induced weight loss in morbid obesity. Obesity (Silver Spring, Md). 14:273-279 (2006).

49. S. Carroll, P. Marshall, and E. Borkoles. Efficacy of lifestyle intervention on peak exercise cardiac power output and reserve in premenopausal obese females: a randomised pilot study. Int J Cardiol. 119:147-155 (2007).

50. E. Kanoupakis, D. Michaloudis, and O. Fraidakis. Left ventricular function and cardiopulmonary performance following surgical treatment of morbid obesity. Obes Surg. 11:552-558 (2001). 
51. R. Gilbert, J.H. Sipple, and J.H. Auchincloss. Respiratory control and work of breathing in obese subjects. J Appl Physiol. 16:21-26 (1961).

52. E. Corpeleijn, W.H. Saris, and E.E. Blaak. Metabolic flexibility in the development of insulin resistance and type 2 diabetes: effects of lifestyle. Obes Rev. 10:178-193 (2009).

53. D. Bond. Bari-active: A preoperative intervention to increase physical activity. Obes Surg. 21 (8):1042 (2011).

54. C. Li, G.S. Zavorsky, D.J. Kim, N.V. Christou, L.S. Feldman, and F. Carli. Effects of a bariatric preoperative exercise program: A pilot randomized study. Surgical Endoscopy and Other Interventional Techniques. 27:S431 (2013). 

PART II: METABOLIC PHYSIOLOGY 


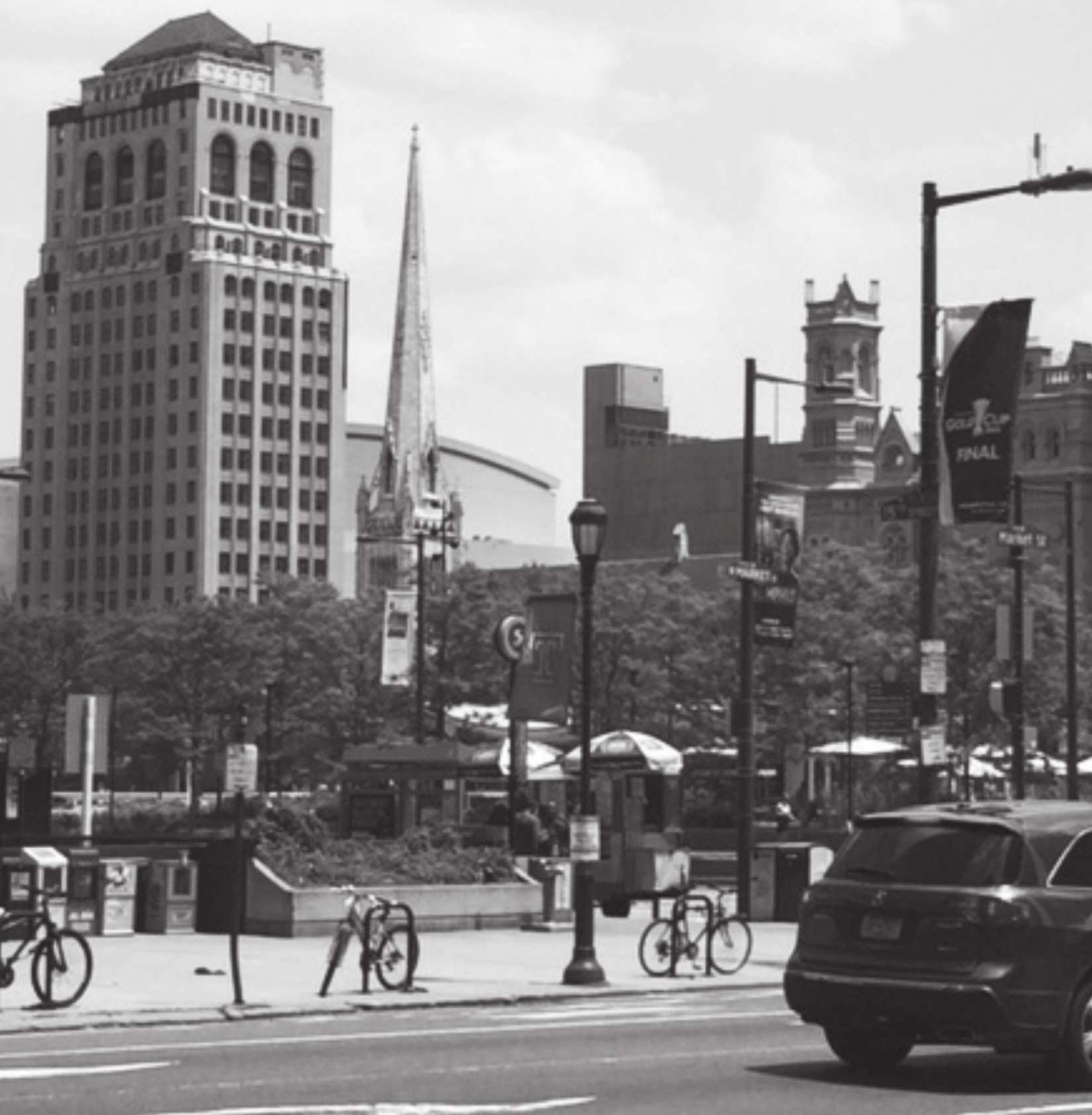




\title{
9. DIFFERENT SUPPLEMENTATION REGIMES TO TREAT PERIOPERATIVE VITAMIN BI2 DEFICIENCIES IN BARIATRIC SURGERY: A SYSTEMATIC REVIEW
}

\author{
Hendrika J.M. Smelt ${ }^{1,2}$ \\ Sjaak Pouwels ${ }^{2,3}$ \\ Johannes F. Smulders ${ }^{2,3}$
}

Obes Surg. 2017 Jan;27(1):254-262

1. Department of Nutrition, Catharina Hospital, Eindhoven, The Netherlands 2. Obesity Center, Catharina Hospital, Eindhoven, The Netherlands 


\section{ABSTRACT}

Vitamin B12 dosage in multivitamin supplementation in the current literature is quite variable. There is no consensus about the optimal treatment of vitamin B12 deficiency.

A systematic literature search on different supplementation regimes to treat peri-operative vitamin B12 deficiencies in bariatric surgery was performed. The methodological quality of 10 included studies was rated using the Newcastle Ottawa scale and ranged from moderate to good. The agreement between the reviewers was assessed with a Cohen's kappa (0.69). The current literature suggests that $350 \mu \mathrm{g}$ oral vitamin B12 is the appropriate dose to correct low vitamin B12 levels in many patients. Further research must focus on a better diagnosis of a vitamin B12 deficiency, the optimal dose vitamin B12 supplementation and clinical relevance next to biochemical data. 


\subsection{INTRODUCTION}

Vitamin B12 deficiencies are common after bariatric surgery. Schilling et al. estimated the prevalence of vitamin B12 deficiency to be 12-33\% (1). Other researchers have suggested a much greater prevalence of vitamin B12 deficiency in up to $75 \%$ of postoperative Roux-en-Y gastric bypass (RYGB) patients. However, most reports have shown approximately $35 \%$ of postoperative RYGB patients as vitamin B12 deficient (1-6). Experts have noted the significance of a functional/subclinical deficiency in the low-normal vitamin B12 range (defined as vitamin B12 levels between 140 and $200 \mathrm{pmol} / \mathrm{l}$ ) that does not exhibit clinical evidence of deficiency. The methylmalonic acid (MMA) assay is the preferred marker of vitamin B12 status because metabolic changes often precede low vitamin B12 levels in the progression to deficiency. The evidence for the optimal vitamin B12 supplementation regimen after bariatric surgery is lacking (9). The dose of vitamin B12 in multivitamin (MV) supplementation in the current literature shows a wide range of variety. There is also no consensus about the optimal treatment of a vitamin B12 deficiency.

This systematic review specifically focuses on vitamin B12 supplementation regimes after bariatric surgery.

To disclose the current scientific knowledge regarding:

- The effect of additional vitamin B12 supplementation in this patient population

- The effect of different vitamin B12 supplementation regimens on blood levels of vitamin B12

\subsection{METHODS}

A systematic multi-database literature search was conducted. The patient population of interest were all patients before or after bariatric surgery. The intervention studied was vitamin B12 supplementation compared to no supplementation (or different supplementation vitamin B12 regimen). Outcome measures were blood levels of vitamin B12.

Pubmed, Embase, Medline, and The Cochrane Library were searched from the earliest date of each database up to December 2015. The search string used for the literature search used the following keywords and was modified for each database: ([bariatric surgery OR metabolic surgery OR sleeve gastrectomy OR roux-en-y gastric bypass OR mini gastric bypass OR omega loop gastric bypass OR biliopancreatic diversion OR duodenal switch OR single anastomosis duodeno-ileal bypass AND vitamin B12 supplementation AND blood levels vitamin B12]). 
Authors HS and SP screened and selected studies on the basis of title and abstract, separately. After primary selection, authors (HS and SP) reviewed the full text of the selected studies and determined suitability for inclusion, based on the established selection criteria. For further eligible studies, cross-references were screened. Disagreements were solved by discussion with each other and the senior author (JS) until consensus was reached.

\section{INCLUSION CRITERIA}

- $\quad$ Randomized controlled trial, prospective or retrospective cohort study

- $\quad$ Patients who were scheduled for bariatric surgery or patients post-bariatric

- $\quad$ All surgical procedures were included (Laparoscopic Gastric banding, Vertical Banded Gastroplasty, Roux-en Y Gastric Bypass, Omega Loop Bypass, Duodenal Switch, biliopancreatic diversion, single anastomosis duodeno-ileal bypass)

- $\quad$ Outcome measure of interest was vitamin B12 levels

\section{EXCLUSION CRITERIA}

- Cross-sectional studies

- $\quad$ Studies looking at pre- and/or postbariatric patients with renal insufficiency

- $\quad$ Postbariatric body contouring surgery and vitamin B12 supplementation

For rating the methodological quality, The Newcastle-Ottawa Scale for non-randomized trials (NOS) was used (10). Stars awarded for each quality item serve as a quick visual assessment. Stars are awarded such that the highest quality studies are awarded up to nine stars. The NOS assigns up to a maximum of nine points for the least risk of bias in three domains: 1) selection of study groups (four points); 2) comparability of groups (two points); and 3) ascertainment of exposure and outcomes (three points) for case-control and cohort studies, respectively.

Two authors (HS and SP) separately assessed the NOS scale of the included studies. A Cohen's kappa score was calculated to determine the level of agreement between authors HS and SP. A Cohen's kappa score $<0.20$ indicates a poor agreement; $0.21-0.40$ a fair agreement; 0.41 0.60 a moderate agreement; $0.61-0.80$ a good agreement; $0.81-1.00$ a very good agreement (16).

All the included vitamin B12 levels were calculated in one general unit (pmol/L), if possible. 


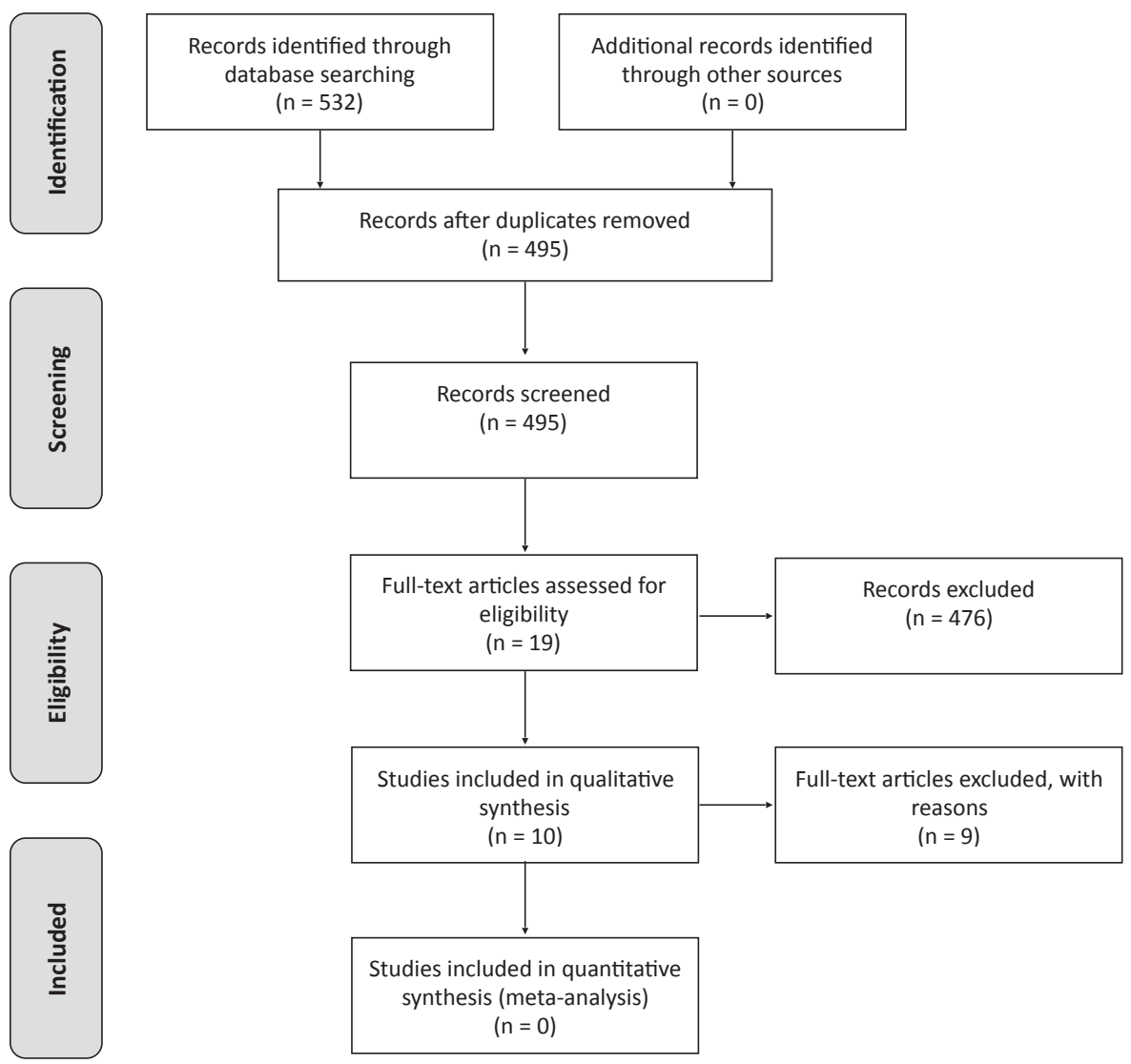

FIGURE 9.1: PRISMA Flowchart 


\subsection{RESULTS}

The primary literature search produced 532 results, including 37 duplicates. After selection on title and abstract, 19 studies were found possibly relevant. Nine studies were excluded, 5 of them were conference abstracts, 2 of them were not online available, 1 study did not use MV supplementation and 1 study consisted of a survey among bariatric surgeons. Due to heterogeneity in patient populations, small sample size of the included studies and lack of standardized reporting of outcome measures (type of supplementation regime and dose of vitamin B12 in the prescribed supplementation), a meta-analysis was not conducted. In total 10 studies were included in this systematic review.

Figure 9.1. outlines our search strategy. The methodological quality of the included studies ranged from moderate to good, indicated by the NOS scale (Table 9.1). A Cohen's kappa of 0.69 reflected a good agreement between authors HS and SP. Table 9.2 gives an overview of the results of the included studies.

\section{STUDY CHARACTERISTICS}

Of all included studies, 1 study was a triple-blind randomized controlled trial (23), 4 studies were prospective cohort studies $(9,11,12,15)$ and 5 studies were retrospective cohort studies $(6,13,14,17,18)$. In total, 10 studies consisted of 1277 participants.

\section{INTERVENTION AND FOLLOW-UP LENGTH}

The length of the intervention ranged from 3 months to 10 years postoperative. Intervention and follow-up length of all studies were described in table 9.3.

\section{VITAMIN B 12 SUPPLEMENTATION}

In 8 studies, the dose of vitamin B12 in the MV supplementation was different (Table 9.4). The studies of Ramos et al., (18) and Brolin et al., (6) lacked of the dose of vitamin B12 of MV supplements.

\section{OUTCOMES LABORATORY TESTS OF VITAMIN B 12}

Outcomes laboratory tests were described in table 9.5. All vitamin B12 levels were calculated in pmol/L, except the studies of Brolin et al., (6) and Ramos et al., (18) which suspectedly used wrong measurement units for indicating a vitamin B12 deficiency. Therefore, the original measurement units of the studies were used in this paragraph. Reference ranges of vitamin B12 levels of all studies were described in table 9.3. 


\section{COMPLAINTS AND CLINICAL EFFECTS}

All included studies did not control the vitamin B12 deficient related complaints. Clinical relevance of the deployed supplementation regime has not been studied in all manuscripts.

TABLE 9.1. Assessment of methodological quality using The Newcastle-Ottawa Scale for non-randomized trials (10).

\begin{tabular}{lccccccccc}
\hline Criteria & S1 & S2 & S3 & S4 & C1 & O1 & O2 & O3 & T \\
\hline Aasheim et al. [11] & $*$ & - & $*$ & $*$ & $* *$ & $*$ & $*$ & $*$ & 8 \\
Brolin et al. [6] & $*$ & - & $*$ & $*$ & $* *$ & $*$ & $*$ & - & 7 \\
Capoccia et al. [13] & $*$ & - & $*$ & $*$ & $* *$ & $*$ & $*$ & $*$ & 8 \\
Dogan et al. [23] & $*$ & $*$ & $*$ & $*$ & $* *$ & $*$ & $*$ & $*$ & 9 \\
Donadelli et al. [9] & $*$ & - & $*$ & $*$ & $* *$ & $*$ & $*$ & - & 7 \\
Gasteyger et al. [17] & $*$ & - & $*$ & $*$ & $* *$ & $*$ & $*$ & - & 7 \\
Homan et al. (2016) & $*$ & $*$ & $*$ & $*$ & $* *$ & $*$ & $*$ & $*$ & 9 \\
Moore et al. (2014) & $*$ & - & $*$ & $*$ & $* *$ & $*$ & - & $*$ & 7 \\
Ramos et al. (2015) & $*$ & - & $*$ & $*$ & $* *$ & $*$ & $*$ & - & 7 \\
Rhode et al. [12] & $*$ & - & $*$ & $*$ & $* *$ & $*$ & - & $*$ & 7 \\
\hline
\end{tabular}

Abbreviations: S1 = Representiveness, S2 = Selection, S3 = Ascertainment, S4 = Demonstration, C1 = Comparability, $\mathrm{O} 1$ = Outcome Selection, $\mathrm{O} 2$ = Outcome Follow-up, O3 = Adequacy 


\begin{tabular}{|c|c|c|}
\hline 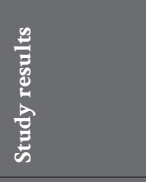 & 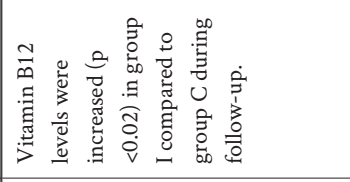 & 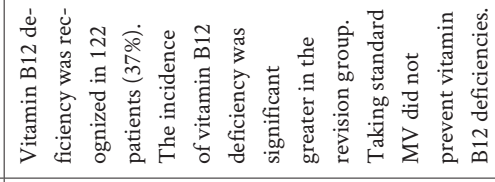 \\
\hline 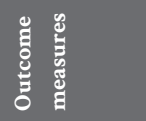 & & 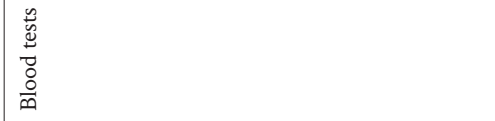 \\
\hline 要 & 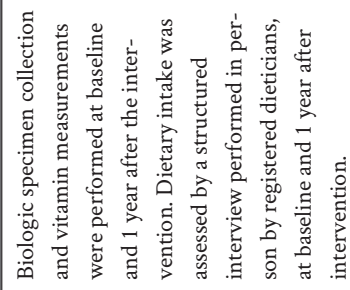 & 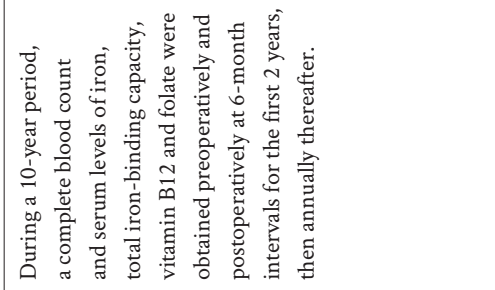 \\
\hline 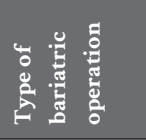 & 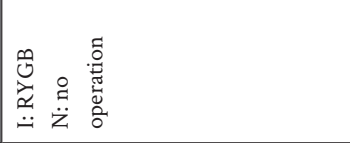 & 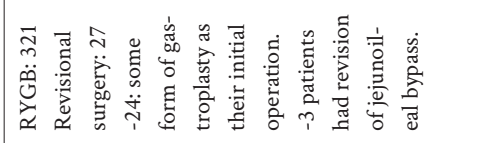 \\
\hline 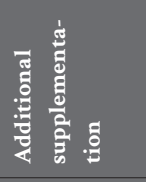 & 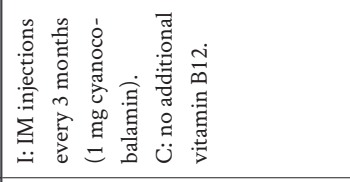 & 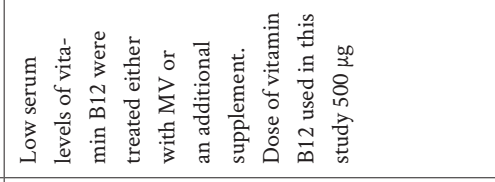 \\
\hline 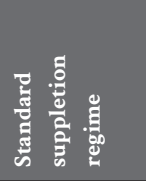 & 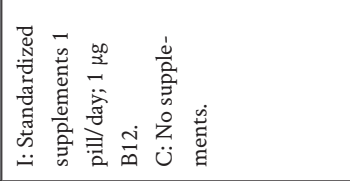 & 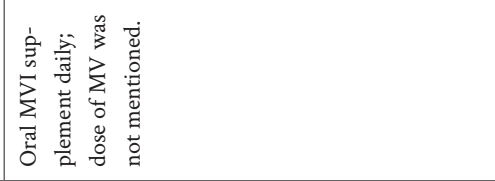 \\
\hline 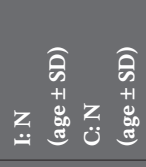 & 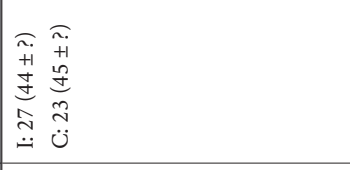 & 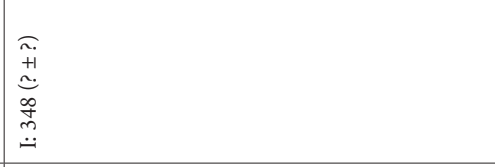 \\
\hline 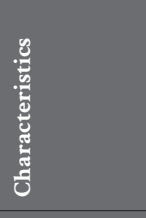 & 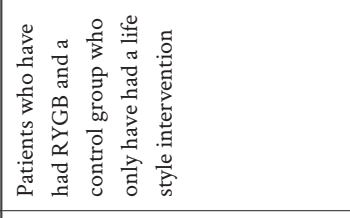 & 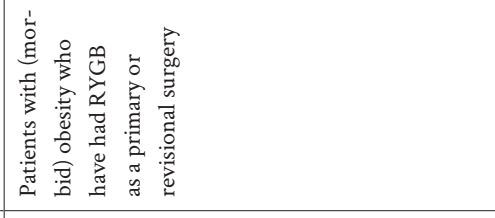 \\
\hline 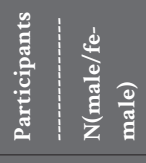 & $\underset{\substack{\infty \\
\infty \\
ٍ}}{\stackrel{0}{\infty}}$ & 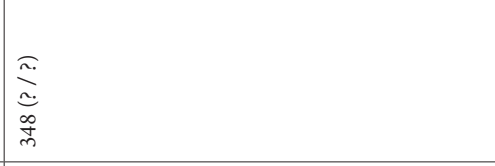 \\
\hline 总 & 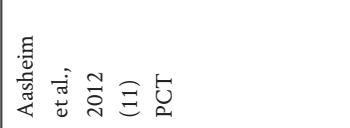 & 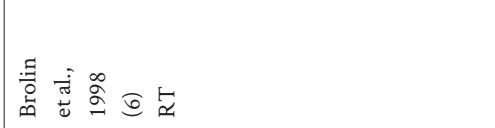 \\
\hline
\end{tabular}




\begin{tabular}{|c|c|c|}
\hline 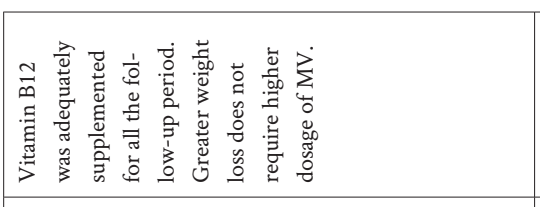 & 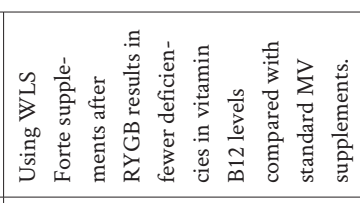 & 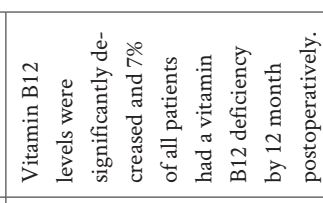 \\
\hline & 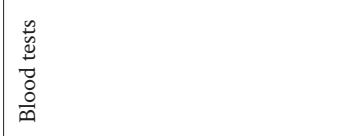 & 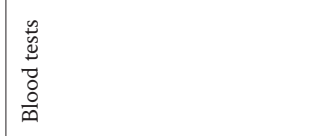 \\
\hline 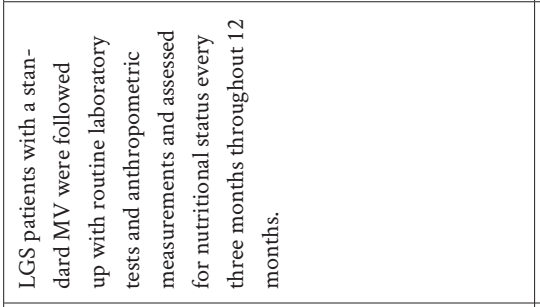 & 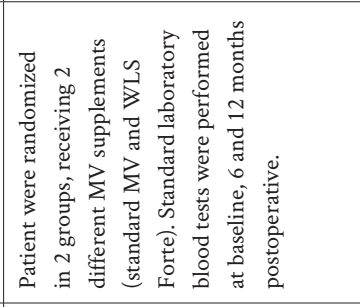 & 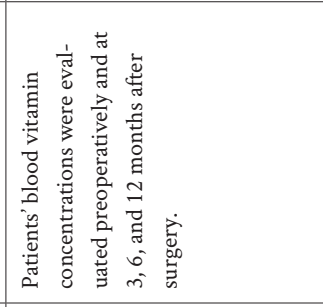 \\
\hline S్ & 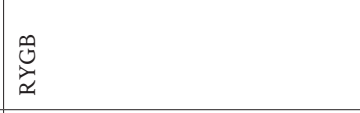 & 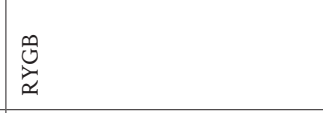 \\
\hline 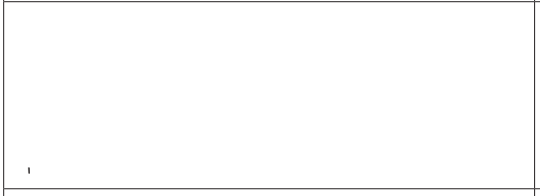 & 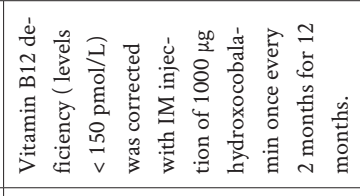 & \\
\hline 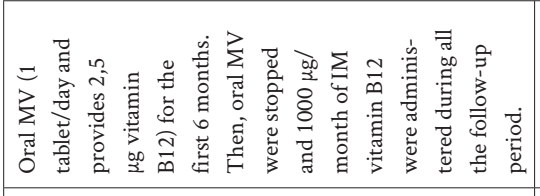 & 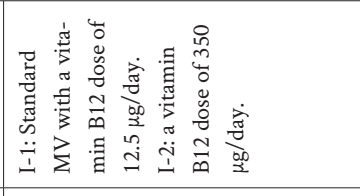 & 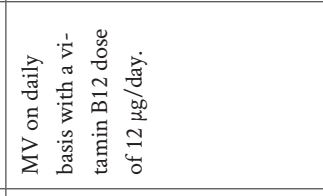 \\
\hline 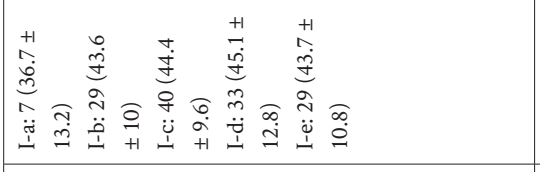 & 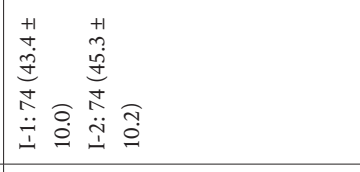 & 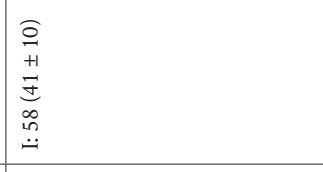 \\
\hline 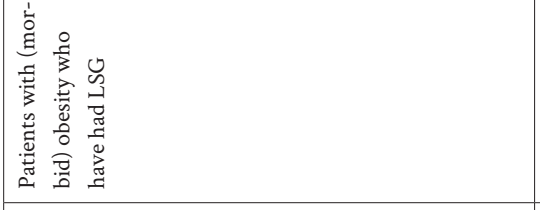 & 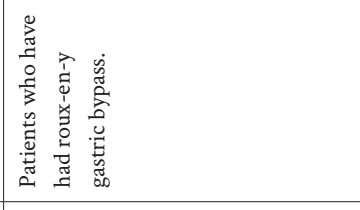 & 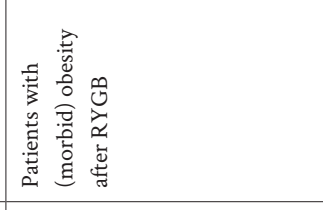 \\
\hline 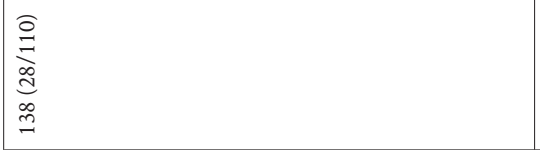 & 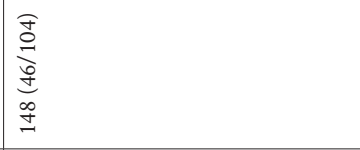 & 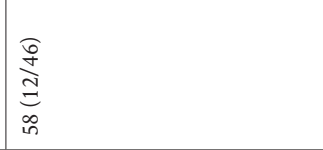 \\
\hline 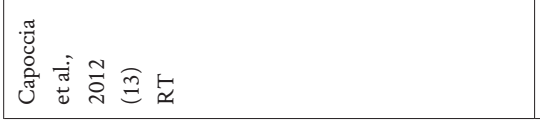 & 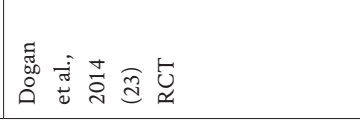 & 产 \\
\hline
\end{tabular}




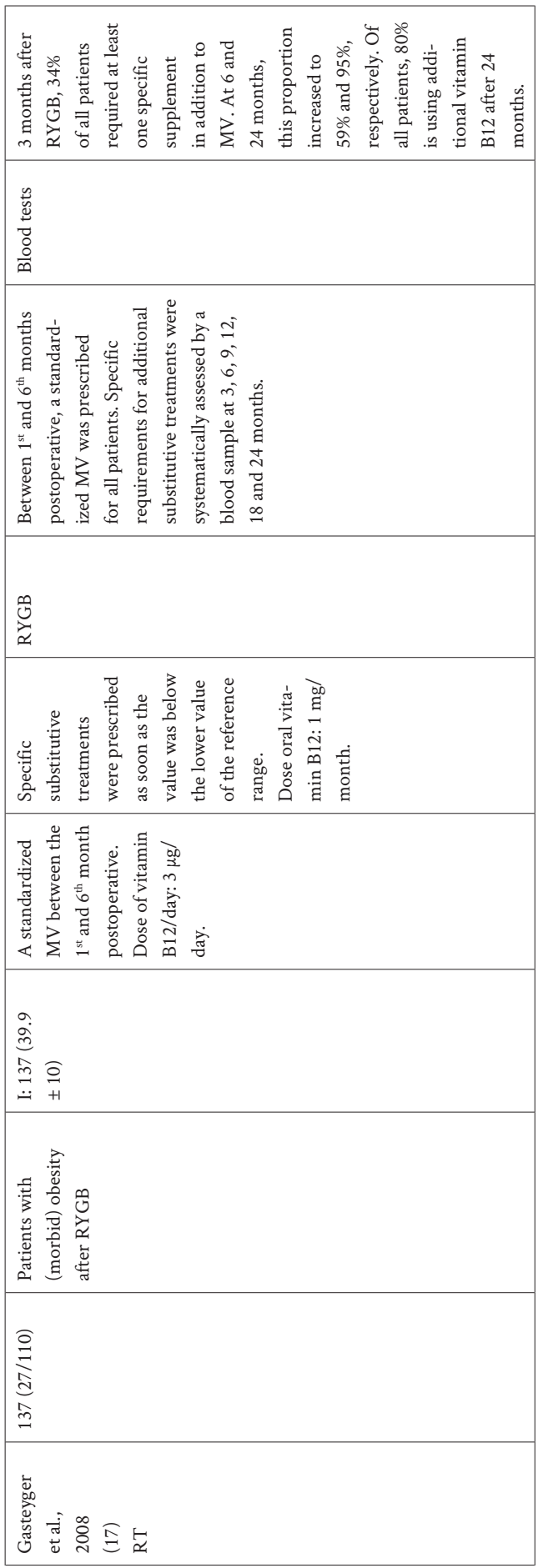




\begin{tabular}{|c|c|}
\hline 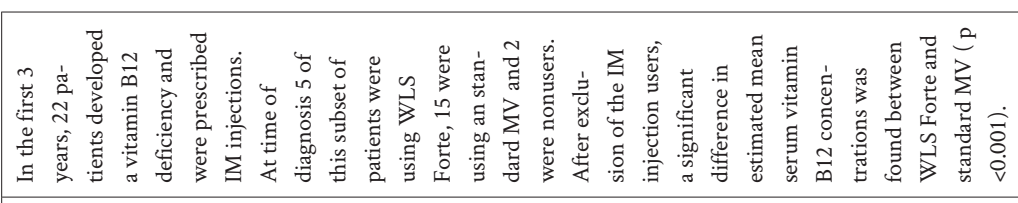 & 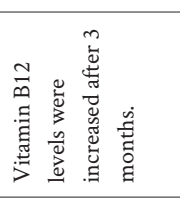 \\
\hline 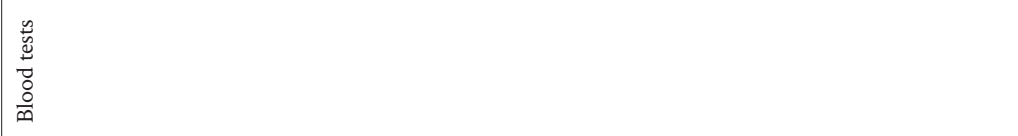 & 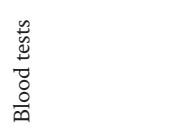 \\
\hline 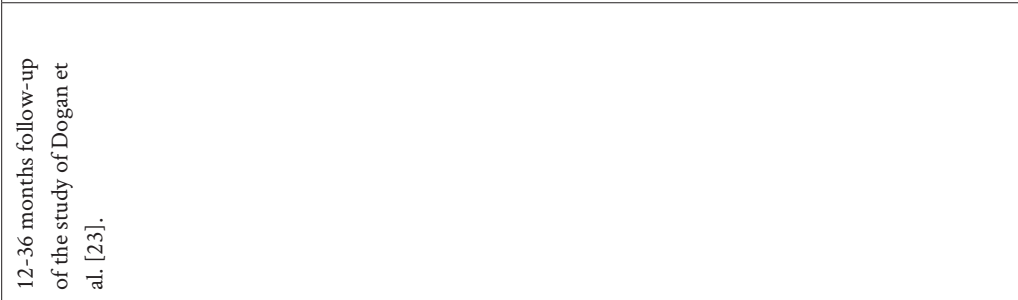 & 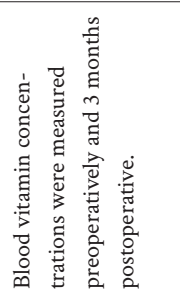 \\
\hline : & 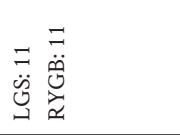 \\
\hline 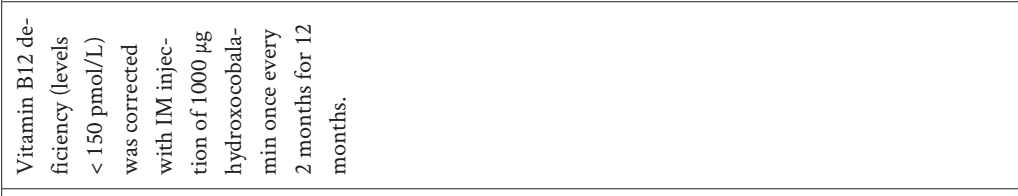 & \\
\hline 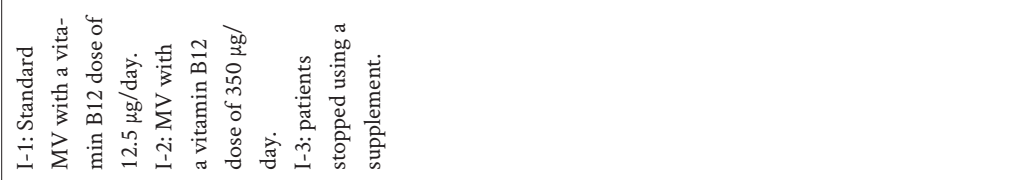 & 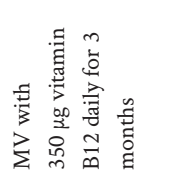 \\
\hline 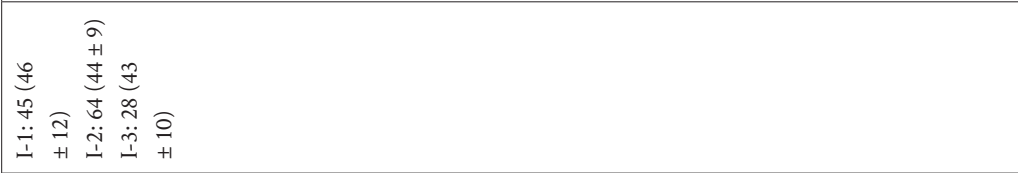 & 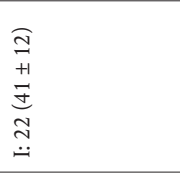 \\
\hline 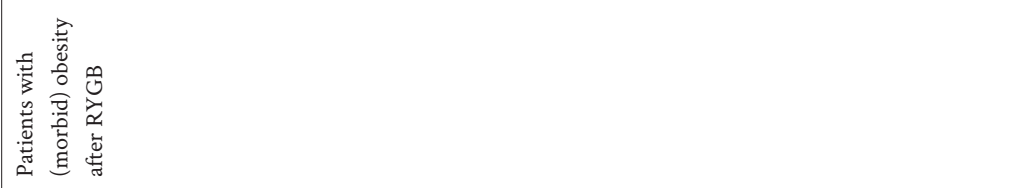 & 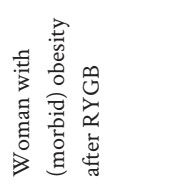 \\
\hline 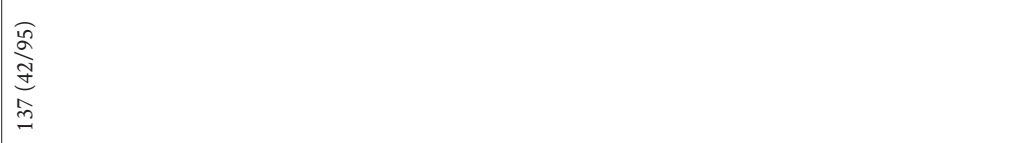 & 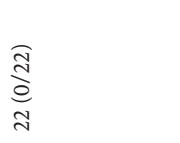 \\
\hline 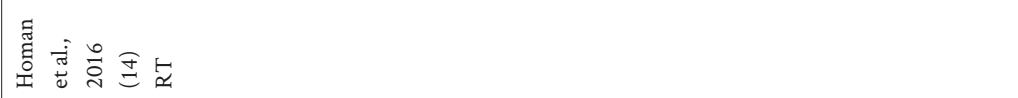 & 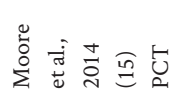 \\
\hline
\end{tabular}


172 CHAPTER 9

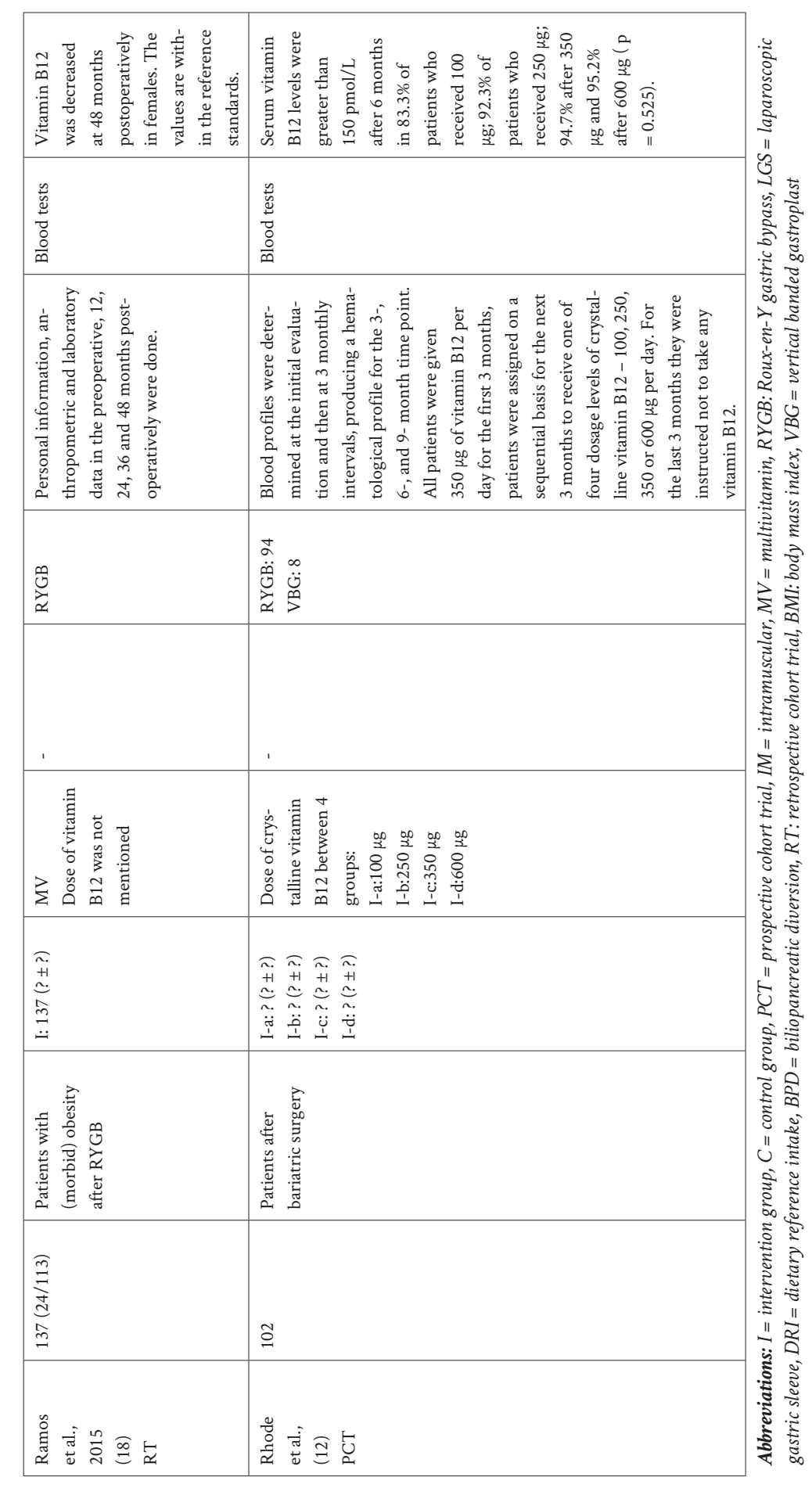


TABLE 9.3. Vitamin B12 supplementation regimen among the included studies

\begin{tabular}{|c|c|}
\hline & Vitamin B12 per day in prescribed supplementation regime \\
\hline Aasheim et al. (11) & $\begin{array}{l}\text { Oral MV: } 1 \mu \mathrm{g} \text { vitamin B12 per day and } 1 \mathrm{mg} \text { IM cyanocobalamin injection every } 3 \\
\text { months }\end{array}$ \\
\hline Capoccia et al. (13) & $\begin{array}{l}\text { First six months: oral MV with 2,5 } \mu \mathrm{g} \text { vitamin B12 per day } \\
\text { After six months: oral MV were stopped and } 1000 \mu \mathrm{g} / \mathrm{month} \text { of IM vitamin B12 were } \\
\text { administered }\end{array}$ \\
\hline Donadelli et al. (9) & Oral MV with $12 \mu \mathrm{g}$ vitamin B12 per day \\
\hline Gasteyger et al. (17) & $\begin{array}{l}\text { First six months: oral MV with } 3 \mu \mathrm{g} \text { vitamin } \mathrm{B} 12 \text { per day } \\
\text { After six months: no MV, substitutive } 1 \mathrm{mg} / \mathrm{month} \text { oral vitamin } \mathrm{B} 12 \text { were prescribed } \\
\text { below the lower reference range. When no satisfactory response was obtained, the } \\
\text { doses were increased to IM injections. Dose of IM injections was not mentioned in the } \\
\text { study. }\end{array}$ \\
\hline Moore et al. (15) & Oral MV with $350 \mu \mathrm{g}$ vitamin B12 per day for 3 months \\
\hline
\end{tabular}

MV: multivitamin, IM: intramuscular, $\mu \mathrm{g}$ : microgram

TABLE 9.4. Dosage of vitamin B12 among the included studies

\begin{tabular}{|c|c|}
\hline & Vitamin B12 per day in prescribed supplementation regime \\
\hline Aasheim et al. (11) & $1 \mu \mathrm{g}$ oral vitamin B12 per day and $1 \mathrm{mg}$ IM injection every 3 months. \\
\hline Brolin et al. (6) & The dose of vitamin B12 in de MV supplements was not mentioned in the study. \\
\hline Capoccia et al. (13) & $\begin{array}{l}\text { First six months: oral MV with 2,5 } \mu \mathrm{g} \text { vitamin } \mathrm{B} 12 \text { per day. } \\
\text { After six months: oral MV were stopped and IM injections with } 1 \mathrm{mg} / \mathrm{month} \text { were } \\
\text { administered. }\end{array}$ \\
\hline Dogan et al. (23) & $\begin{array}{l}\text { I-1:supplement with } 350 \mu \mathrm{g} \text { vitamin B12. } \\
\text { I-2: standard MV supplementation with } 12,5 \mu \mathrm{g} \text { vitamin B12. Treatment of a } \\
\text { deficiency: IM injections with } 1 \mathrm{mg} \text { per } 2 \text { months for } 12 \text { months. }\end{array}$ \\
\hline Donadelli et al. (9) & $12 \mu \mathrm{g}$ oral vitamin B12 per day. \\
\hline Gasteyger et al. (17) & $\begin{array}{l}\text { First six months: } 3 \mu \mathrm{g} \text { oral vitamin B12 per day. } \\
\text { After six months: no MV, substitutive } 1 \mathrm{mg} / \mathrm{month} \text { oral vitamin B12 were prescribed } \\
\text { below the lower reference range. When no satisfactory response was obtained, the } \\
\text { doses were increased to IM injections. Dose of IM injections was not mentioned in the } \\
\text { study. }\end{array}$ \\
\hline Homan et al. (14) & $\begin{array}{l}\text { I-1: supplement with } 350 \mu \mathrm{g} \text { vitamin B12. } \\
\mathrm{I}-2 \text { : standard MV supplementation with } 12,5 \mu \mathrm{g} \text { vitamin B12. Treatment of a deficiency: } \\
\text { IM injections with } 1 \mathrm{mg} \text { per } 2 \text { months for } 12 \text { months. }\end{array}$ \\
\hline Moore et al. (15) & $350 \mu \mathrm{g}$ oral vitamin B12 per day for 3 months. \\
\hline Ramos et al. (18) & The dose of vitamin B12 in de MV supplements was not mentioned in the study. \\
\hline Rhode et al. (12) & $\begin{array}{l}\text { First } 3 \text { months: } 350 \mu \mathrm{g} \text { oral vitamin B12 per day. Thereafter, in the following oral } \\
\text { dosage for the next } 3 \text { months: } \\
\text { I-1: } 100 \mu \mathrm{g} \text {. } \\
\text { I-2: } 250 \mu \mathrm{g} \text {. } \\
\text { I-3: } 350 \mu \mathrm{g} \text {. } \\
\text { I-4: } 600 \mu \mathrm{g} \text {. }\end{array}$ \\
\hline
\end{tabular}

MV: multivitamin, I-1-4: intervention group 1-4, IM: intramuscular, $\mu \mathrm{g}$ : microgram, RDA: recommended daily allowance. 
TABLE 9.5. Outcomes laboratory tests of vitamin $B 12$

\begin{tabular}{|c|c|}
\hline Aasheim et al. (11) & $\begin{array}{l}\text { Vitamin B12 levels increases after intervention, compared with the control group ( } \mathrm{P}< \\
0.02 \text { ). Of } 2 \text { patients who developed vitamin B12 deficiency (compared with non in the } \\
\text { control group), } 1 \text { reported not having had IM injections. Actual vitamin B12 levels were } \\
\text { not mentioned in the study. }\end{array}$ \\
\hline Brolin et al. (6) & $\begin{array}{l}\text { Vitamin B12 levels were significantly lower than mean preoperative values only at } 12 \\
\text { and } 24 \text { months after surgery. } 37 \% \text { of the patients had a vitamin B12 deficiency and } \\
\text { the incidence of vitamin B12 deficiency after surgery was significantly greater in the } \\
\text { revision group ( } \mathrm{p} \leq 0.004 \text { ). There was no correlation between regular ingestion of MV } \\
\text { supplementation and the potential for developing a vitamin B12 deficiency. In this } \\
\text { study more than } 80 \% \text { of the vitamin B12 deficiencies responded to oral supplementation } \\
\text { (containing } 500 \mu \mathrm{g} \text { vitamin B12). } \\
\text { Actual vitamin B12 levels were measured but not mentioned in the study. }\end{array}$ \\
\hline Capoccia et al. (13) & $\begin{array}{l}\text { Vitamin B12 was adequately supplemented for all the follow-up period (before surgery and } \\
12 \text { months after surgery, } 365.8 \pm 193.7 \mathrm{pmol} / \mathrm{L} \text { and } 360.8 \pm 169.0 \mathrm{pmol} / \mathrm{L}, \text { respectively). } \\
\text { Baseline vitamin B12 levels: } 317.3 \pm 132.8 \mathrm{pmol} / \mathrm{L}, 286.0 \pm 188.7 \mathrm{pmol} / \mathrm{L}, 376.8 \pm \\
197.8 \mathrm{pmol} / \mathrm{L}, 517.3 \pm 191.0 \mathrm{pmol} / \mathrm{L}, 258.8 \pm 303.5 \mathrm{pmol} / \mathrm{L} \text {, for group A, B, C, D and E } \\
\text { respectively. Follow up results } 12 \mathrm{months} \text { after surgery: } 338.7 \pm 284.4 \mathrm{pmol} / \mathrm{L}, 349.8 \pm \\
193.3 \mathrm{pmol} / \mathrm{L}, 268.6 \pm 119.0 \mathrm{pmol} / \mathrm{L}, 284.4 \pm 154.3 \mathrm{pmol} / \mathrm{L}, 303.5 \pm 187.0 \mathrm{pmol} / \mathrm{L} \text {, for } \\
\text { group A, B, C, D and E respectively. } \\
\text { Percentage deficiencies were not mentioned in the study. }\end{array}$ \\
\hline Dogan et al. (23) & $\begin{array}{l}\text { At baseline, vitamin B12 deficiency was diagnosed in } 9(6.1 \%) \text { patients, that is, } 5(6.8 \%) \\
\text { patients in the standard MV group and } 4(5.44 \%) \text { patients in the high dose group. These } \\
\text { patients received IM injections by protocol. In total, } 27(18.2 \%) \text { additional patients were } \\
\text { treated with IM injections at any time during the } 12 \text { month follow-up: } 17(23 \%) \text { in the } \\
\text { standard MV group and } 10(13.5 \%) \text { in the high dose group ( } \mathrm{p}=0.14) \text {. The results obtained } \\
\text { after exclusion of these patients receiving IM injections: mean vitamin B12 serum levels } \\
\text { decreased by } 38.9 \pm 141.3 \mathrm{pmol} / \mathrm{L} \text { in the standard MV group and increased by } 44.1 \pm 138.8 \\
\text { pmol/L in the high dose group ( } \mathrm{p}<0.001) \text { after } 12 \text { months, and as a result mean vitamin } \\
\text { B12 blood serum levels at } 6 \text { months and } 12 \text { months were significantly higher with high } \\
\text { dose compared with standard MV ( } \mathrm{p}<0.05) \text {. After } 12 \text { months, vitamin B12 deficiency had } \\
\text { developed in } 5(7.9 \%) \text { patients receiving standard MV versus } 1(1.6 \%) \text { patient in the high } \\
\text { dose group }(\mathrm{p}=0.207) \text {. }\end{array}$ \\
\hline Donadelli et al. (9) & $\begin{array}{l}\text { Vitamin B12 levels remained constant up to } 3 \text { months }(331.7 \pm 183.9 \mathrm{pmol} / \mathrm{L}) \text { until } 6 \\
\text { months }(295.8 \pm 183.0 \mathrm{pmol} / \mathrm{L}) \text { after surgery but were significantly decreased after } 12 \\
\text { months }(274.9 \pm 196.9 \mathrm{pmol} / \mathrm{L}, \mathrm{p}=<0.05 \text { versus basal). } \\
7 \% \text { of patients had vitamin B12 deficiency } 1 \text { year after surgery. }\end{array}$ \\
\hline $\begin{array}{l}\text { Gasteyger et al. } \\
(17)\end{array}$ & $\begin{array}{l}10 \% \text { of all patients used additional vitamin B12 at } 3 \text { months, } 28 \% \text { at } 6 \text { months, } 62 \% \text { at } 12 \\
\text { months, } 72 \% \text { at } 18 \text { months and } 80 \% \text { at } 24 \text { months. }\end{array}$ \\
\hline Homan et al. (14) & $\begin{array}{l}\text { In the first } 3 \text { years, } 22 \text { patients developed a vitamin B12 deficiency and were prescribed IM } \\
\text { injections. At time of diagnosis } 5 \text { of this subset of patients were using high dose vitamin } \\
\text { supplements, } 15 \text { were using a standard MV and } 2 \text { were nonusers. The difference in IM } \\
\text { injections use between high dose vitamin supplements }(409 \pm 25) \text { and standard MV ( } 330 \\
\pm 27 \text { ) was significant ( } \mathrm{p}=0.001) \text {. After exclusion of the IM injection users, a significant } \\
\text { difference in estimated mean serum vitamin B12 was found between high dose vitamin } \\
\text { supplements }(335 \pm 12) \text { and standard MV }(264 \pm 12)(\mathrm{p}<0.001) \text {. } \\
\text { In total } 7 \text { patients were diagnosed with a vitamin B12 deficiency at } 36 \text { months ( } 3 \text { in the } \\
\text { standard MV group and } 4 \text { in the nonusing group). Combining the IM injection users } \\
\text { and the deficient patients resulted in a total of } 29 \text { patients with a vitamin B12 deficiency } \\
\text { throughout the study ( } 5 \text { while using high dose vitamin supplements, } 18 \text { while using } \\
\text { standard MV and } 6 \text { while using no supplement). The difference between high dose vitamin } \\
\text { supplements and standard MV was significant ( }<0.001) \text {. }\end{array}$ \\
\hline
\end{tabular}




\begin{tabular}{|c|c|}
\hline Moore et al. (15) & $\begin{array}{l}\text { High dose MV supplementation daily for } 3 \text { months resulted in a } 48 \% \text { increase of serum } \\
\text { vitamin } \mathrm{B} 12 \text {. A significant increase was seen in all patients after LSG (from } 356.5 \pm 93.0 \\
\text { pmol/L to } 466.4 \pm 220.7 \mathrm{pmol} / \mathrm{L}, \mathrm{p}=0.0336 \text { ) and in all patients after RYGB (from } 377.1 \pm \\
129.2 \mathrm{pmol} / \mathrm{L} \text { to } 605.9 \pm 295.2 \mathrm{pmol} / \mathrm{L}, \mathrm{p}=0.033 \text { ). }\end{array}$ \\
\hline Ramos et al. (18) & $\begin{array}{l}\text { Male: } \\
\text { Preoperative: } 464.0 \pm 140.6 \mathrm{mg} / \mathrm{dL} \text {. } \\
\text { Postoperative: } 12 \text { months: } 373.8 \pm 148.3 \mathrm{mg} / \mathrm{dL}, 24 \text { months: } 317.8 \pm 163.7 \mathrm{mg} / \mathrm{dL}, 36 \\
\text { months } 401.4 \pm 352.0 \mathrm{mg} / \mathrm{dL}, 48 \text { months: } 354.4 \pm 186.6 \mathrm{mg} / \mathrm{dL} \text {. } \\
\text { Female: } \\
\text { Preoperative: } 512.5 \pm 561.5 \mathrm{mg} / \mathrm{dL} \text {. } \\
\text { Postoperative: } 12 \mathrm{months:} 395.6 \pm 247.0 \mathrm{mg} / \mathrm{dL}, 24 \mathrm{months:} 391.5 \pm 212.9 \mathrm{mg} / \mathrm{dL}, 36 \\
\text { months } 351.3 \pm 177.1 \mathrm{mg} / \mathrm{dL}, 48 \text { months: } 395.8 \pm 220.3 \mathrm{mg} / \mathrm{dL} . \\
\text { Percentage vitamin B12 deficiencies was not mentioned in the study. }\end{array}$ \\
\hline Rhode et al. (12) & $\begin{array}{l}\text { Serum vitamin B12 levels were }<100 \mathrm{pmol} / \mathrm{L} \text { at baseline and greater than } 150 \mathrm{pmol} / \mathrm{L} \text { after } \\
6 \text { months in } 83.3 \% \text { of patients who received } 100 \mu \mathrm{g} ; 92.3 \% \text { of patients who received } 250 \mu \mathrm{g} \text {; } \\
94.7 \% \text { after } 350 \mu \mathrm{g} \text { and } 95.2 \% \text { after } 600 \mu \mathrm{g}(\mathrm{p}=0.525) \text {. }\end{array}$ \\
\hline
\end{tabular}

\subsection{DISCUSSION}

This systematic review highlights the current evidence on the effects of MV or additional vitamin B12 supplementation in patients after bariatric surgery. Vitamin B12 supplementation has an effect on the intracellular vitamin B12 content and in the optimal dosage it can prevent a vitamin B12 deficiency. However, vitamin B12 deficiencies preoperatively are not uncommon in morbidly obese people. In the study of Dogan et al., (23) a vitamin B12 deficiency was diagnosed in 9 patients $(6.1 \%)$ and 3 patients $(5.2 \%)$ in the study of Donadelli et al., (9) had a vitamin B12 deficiency in the preoperative period. This is not clearly reported in the other 8 studies.

There is no consensus about the optimal dosage of vitamin B12 supplementation after bariatric surgery worldwide. ASMBS guidelines advise oral vitamin B12 supplements of 350 to $500 \mu \mathrm{g}$, and if necessary, intramuscular (IM) injections of $1000 \mu \mathrm{g}$ per month (24). The ACCE/TOS/ASMBS guidelines advise oral supplementation with crystalline vitamin B12 at a dosage of $1000 \mu \mathrm{g}$ daily or more may be used to maintain normal vitamin B12 levels. Intranasally administered vitamin B12, $500 \mu \mathrm{g}$ weekly, may also be considered. Parenteral (IM or subcutaneous) vitamin B12 supplementation, $1000 \mu \mathrm{g} / \mathrm{month}$ to $1000-3000 \mu \mathrm{g}$ every 6 to 12 months, is indicated if vitamin B12 sufficiency cannot be maintained using oral or intranasal routes $(25)$.

However, definitive conclusions cannot be made after this systematic review, because of the heterogeneity of MV supplementation or additional vitamin B12 IM injection regimes and 
timing of this additional vitamin B12 IM injections. Besides that, all the included studies did not control the vitamin B12 deficient related complaints. Clinical relevance has not been studied in all manuscripts. These data are needed to examine whether biochemical benefits of vitamin B12 supplementation are correlated with clinical improvement.

Two interesting findings were found in the included studies. First, in 4 included studies a dose of $350 \mu \mathrm{g}$ vitamin B12 per day was using $(12,14,15,23)$. In the study of Moore et al., (15) vitamin B12 levels of all patients were increased 3 months postoperatively. In the study of Dogan et al., (23) high dose vitamin B12 supplements results in fewer vitamin B12 deficiencies compared with standard MV supplements. The study of Homan et al., (14) showed that high dose vitamin B12 supplements is effective as MV supplement that is able to reduce the number of patients with vitamin B12 deficiencies, compared with a standard MV supplement. In the study of Rhode et al., (12) serum levels of vitamin B12 were $>150 \mathrm{pmol} / \mathrm{L}$ after 6 months in $95 \%$ of the patients.

Secondly, all the other studies used MV supplementation with a dose of vitamin B12 ranging from 3 to $12 \mu \mathrm{g}$ per day $(9,17)$ or unknown dose of vitamin B12 $(6,18)$. The studies of Brolin et al., Donadelli et al. and Gasteyger et al., showed many vitamin B12 deficiencies in the follow up $(6,9,17)$. Contrary results were found in the study of Ramos et al., (18), the vitamin B12 levels are within the reference standards. However, the dose of vitamin B12 in his study was unknown.

\section{VITAMIN B 12 SUPPLEMENTATION}

Dose of vitamin B12 in the MV supplementation in all included studies, is varied from 1 $\mu \mathrm{g} /$ day to high dose supplementation with 350-600 $\mu \mathrm{g} /$ day. MV supplements with $350 \mu \mathrm{g}$ vitamin B12 per day can maintain normal-high vitamin B12 levels in many patients (12, 14, $15,23)$. The body's storage will be depleted much faster in patients who using standard MV supplementation with a low dose of vitamin B12. Of the included studies, the supplementation regimes with an oral vitamin B12 dosage $<350 \mu \mathrm{g} /$ day, eight of the included studies showed persistence of deficiencies even after a period supplementation of vitamin B12. $(6,9,11$ $14,17,23)$ In one study (6) no dosage of vitamin B12 was measured, in the other studies the dosage of vitamin B12 was lower than < $350 \mu \mathrm{g} /$ day. $(9,11-14,17,23)$ In 2 included studies, IM injections are belong to the standard MV supplementation regime with a low dose of vitamin B12 $(11,13)$. Dose of the IM injections is equal but the frequency of given IM injections is also different (table 4). In the study of Aasheim et al., (11) 2 patients developed a vitamin B12 deficiency and in the study of Capoccia et al., (13) vitamin B12 levels decreased in many patients, which suggests that this both regimen were not optimal. 
If $\mathrm{MV}$ supplementation with a high dose of vitamin B12 may improve the vitamin B12 levels in many patients, IM injections as a standard regime is not necessary and because of this, many patients were unnecessary loaded with IM injections. This seems like contradictory advice, but to determine whether IM injections of vitamin B12 are necessary, we need focus on the combination vitamin B12 and MMA. This is essential to assess whether there is an absolute of a functional deficiency of vitamin B12, because of the failure rate of the current vitamin B12 assays between 22-35\% (7, 8, 19, 20). Therefore whether it is necessary to add IM injections to the standard supplementation regime. Besides that, these data are subjectively and it is unclear if patients take their supplements daily. Life-long compliance of daily supplement intake is hard to achieve. To measure adequate intake of MV supplementation, one can monitor the serum concentration of highly absorbable vitamins. Some investigators have reported that low folate levels reflect nonadherence to MV supplementation because the amount of supplemented folic acid properly corrects low serum folate levels $(9,23)$. Only two studies have looked at compliance of MV supplementation intake and distinction in processing these data $(14,23)$.

\section{OUTCOMES LABORATORY TESTS OF VITAMIN B 12 AND MMA}

Vitamin B12 assays that are currently used to diagnose clinical vitamin B12 deficiency have a failure rate of $22-35 \%(7,8,19,20)$. This failure rate may be due to the fact that $80 \%$ of the vitamin B12 in plasma is bound to the transport protein haptocorrin. This percentage is biologically unavailable and cannot be absorbed by the cells, which means that plasma vitamin B12 concentrations poorly correlate with the bioavailable intracellular vitamin B12 content $(8,21,22)$. Measuring vitamin B12 is a poor predictor for a functional vitamin B12 status. In the study of Smelt et al., (8) more vitamin B12 deficiencies were found if MMA is included in the diagnosis. When a vitamin B12 level is between 140 and $200 \mathrm{pmol} / \mathrm{l}$, additional MMA levels should be measured to determine whether there is a functional vitamin B12 deficiency. In this review, no included study used the additional parameter MMA. Given the high failure rate of vitamin B12 assays, many vitamin B12 deficiencies will be untreated.

\section{STUDY LIMITATIONS}

First, the following limitations are present when evaluating the literature: 1) heterogeneous patient populations being studied, 2) non-comparable vitamin B12 from supplements being evaluated, 3) lack of many data (some studies lacked of gender, age, reference range of vitamin B12 levels, actual vitamin B12 levels after intervention, lack of dose of vitamin B12 in MV supplementation) and 4) lack of well-designed prospective cohort and randomized controlled studies for the right use of vitamin B12 in post bariatric patients. Secondly, only biochemical data was measured and clinical relevance was not demonstrated. 


\subsection{CONCLUSION}

In bariatric surgery, vitamin B12 deficiencies have a high prevalence. Unfortunately there is no consensus about multivitamin supplementation and any additional vitamin B12 supplementation. The current literature suggests that $350 \mu \mathrm{g}$ of oral vitamin B12 is the appropriate oral dose to correct low serum vitamin B12 levels in many patients. A lifelong follow-up regimen seems necessary, because MV supplementation with a high dose of vitamin B12 cannot prevent all deficiencies. Further research must focus on a better diagnosis of a vitamin B12 deficiency with possible additional parameters like MMA, the right dose of vitamin B12 supplementation and the clinical relevance beside biochemical data. 


\subsection{REFERENCES}

1. Schilling R.D., Gohdes P.N., Hardie G.H. Vitamin B12 deficiency after gastric bypass for obesity. Ann Intern Med.1984; 101: 501-502.

2. Kushner R. Managing the obese patient after bariatric surgery: a case report of severe malnutrition and review of the literature. JPEN J Parenter Enteral Nutr. 2000; 24: 126-132.

3. Brolin R.E.,LaMarca L.B., Henler H.A., Cody R.P. Malabsorptive gastric bypass in patients with super-obesity. J Gastrointest Surg. 2002; 6: 195-203.

4. Rhode B.M., Arseneau P., Cooper B.A., Katz M., Gilfix B.M., MacLean L.D. Vitamin B12 deficiency after gastric surgery for obesity. Am J Clin Nutr. 1996; 63: 103-109.

5. MacLean L.D., Rhode B.M., Shizgal H.M. Nutrition following gastric operations for morbid obesity. Ann Surg. 1983; 198: 347-355.

6. Brolin R.E., Gorman J.H., Gorman R.C., et al. Are vitamin B12 and folate deficiency clinically important after roux-en-y gastric bypass? J Gastrointest Surg. 1998; 2 (5): 436-442.

7. Sumner A.E. Elevated methylmalonic acid and total homocysteine levels show high prevalence of vitamin B12 deficiency after gastric surgery. Ann Intern Med. 1996; 124: 469-476.

8. Smelt H.J.M., Smulders J.F., Said M., Nienhuijs S.W., Boer A.K. Improving bariatric patient aftercare outcome by improved detection of a functional vitamin B12 deficiency. Obes Surg. 2015. DOI: $10.1007 / \mathrm{s} 11695-015-1952-8$.

9. Donadelli S.P., Junqueira-Franco M.V.M., de Mattos Donadelli C.A., et al. Daily vitamin supplementation and hypovitaminosis after obesity surgery. Nutr. 2012; 28 (4): 391-396.

10. Wells G.A., Shea B., O'Conell D., et al. The Newcastle-Ottawa Scale (NOS) for assessing the quality of nonrandomized studies in meta-analysis.

11. Aasheim E.T., Johnson L.K., Hofso D., Bohmer R., Hielmeseath J. Vitamin status after gastric bypass and lifestyle intervention: a comparative prospective study. Surg Obes Relat Dis. 2012; 8 (2): 169-175.

12. Rhode B.M., Tamin H., Gilfix B.M., Sampalis J.S., Nohr C., MacLean L.D. Treatment of vitamin B12 deficiency after gastric surgery for severe obesity. Obes Surg. 1995; 5 (2): 154-158.

13. Capoccia D., Coccia F., Paradiso F., et al. Laparoscopic gastric sleeve and micronutrients supplementation: our experience. J of Obes. 2012. DOI: 10.1155/2012/672162.

14. Homan J., Schijns W., Aarts E.O., Laarhoven van C.J.H.M., et al. An optimized multivitamin supplement lowers the number of vitamin and mineral deficiencies three years after roux-en-y gastric bypass: a cohort study. Surg Obes Rel Dis. 2015. DOI: 10.1016/j.soard.2015.12.010.

15. Moore C.E., Sherman V. Effectiveness of B vitamin supplementation following bariatric surgery: rapid increases of serum vitamin B12. Obes Surg. 2015; 25: 694-699.

16. Altman D. Practical statistics for medical research. Chapman and Hal. London. 1991.

17. Gasteyger C., Suter M., Gaillard R.C., Giusti V. Nutritional deficiencies after roux-en-y gastric bypass for morbid obesity often cannot be prevented by standard multivitamin supplementation. Am J Clin Nutr. 2008; 87: 1128-1133. 
18. de Jesus Ramos N.M.C.P., Magno F.C.C.M., Cohen L., Rosado E.L., Carneiro J.G.I. Weight loss and nutritional anemia in patients submitted to roux-en-y gastric bypass on use of vitamin and mineral supplementation. Arq Bras Cir Dig. 2015; 28 (1): 44-47.

19. Carmel R, Brar S, Agrawal A, Penha P.D. Failure of assay to identify low cobalamin concentrations. Clin Chem. 2000; 46 (12): 2017-2025.

20. Carmel R, Agrawal YP. Failures of cobalamin assays in pernicious anemia. N Engl J Med. 2012; 367: 385-391.

21. Solomon L.R. Disorders of cobalamin metabolism: emerging concepts in pathophysiology, diagnosis and treatment. Blood Rev. 2007; 3: 113-130.

22. Hvas A.M., Nexo E. Holotranscobalamin: a first choice assay for diagnosing early vitamin B12 deficiency? J Int Med. 2005; 257: 289-298.

23. Dogan K., Aarts E.O., Koehestanie P. et al. Optimization of vitamin supplementation after rouxen-y gastric bypass surgery can lower postoperative deficiencies: a randomized controlled trial. Medicine. 2014; 93 (25).

24. Aills L, Blankenship J, Buffington C, et al. ASMBS Allied health nutritional guidelines for surgical weight loss patient. Surgery for obesity and related diseases. 2008; 4: 73108.

25. Mechanick J.I., Youdim A., Jones D.B., et al. Clinical practice guidelines for the perioperative nutritional, metabolic, and nonsurgical support of the bariatric surgery patient - 2013 update. Endocr Pract. 2013; 19 (2). 



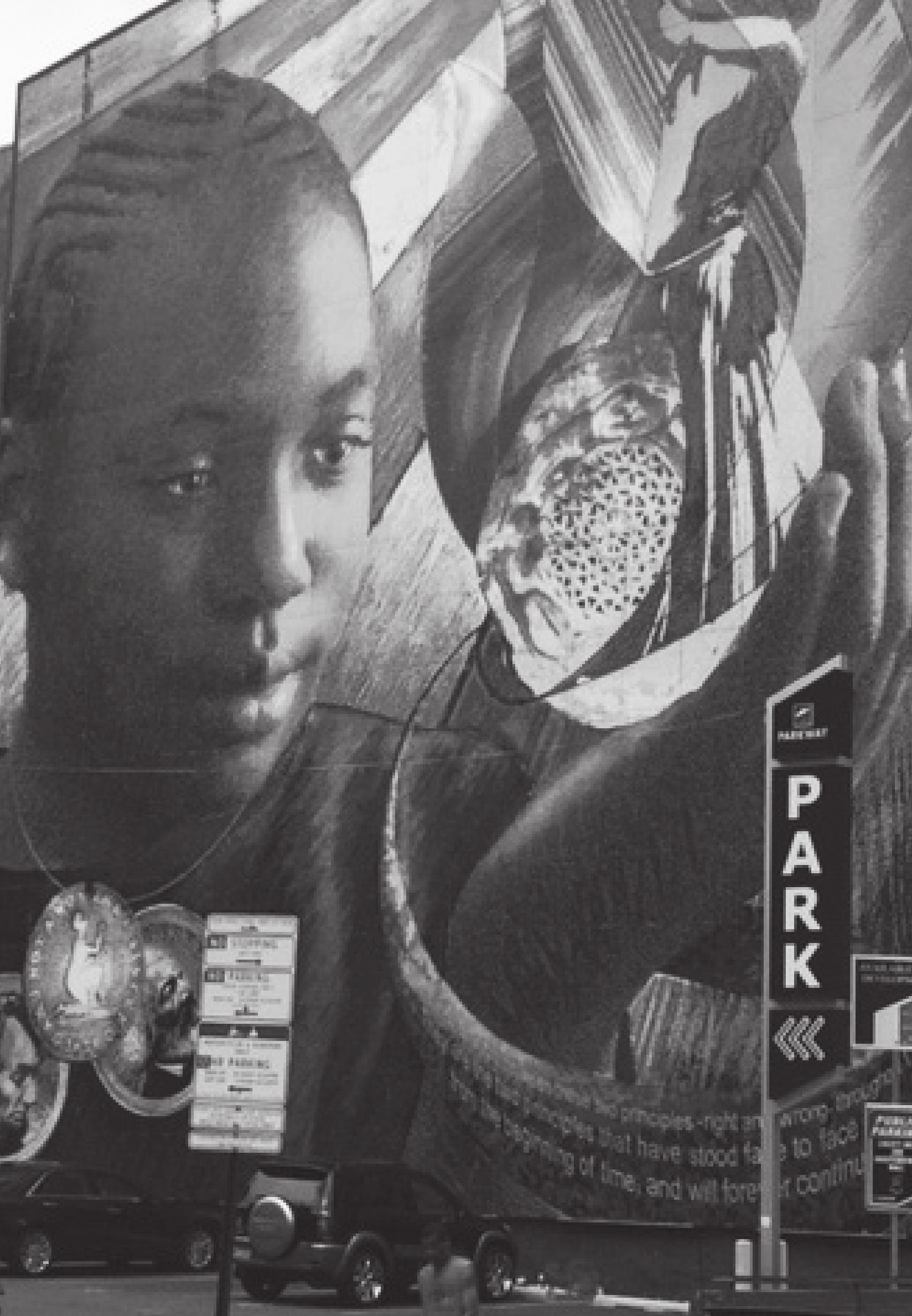




\title{
10. COMPARISON BETWEEN DIFFERENT INTRAMUSCULAR VITAMIN B12 SUPPLEMENTATION REGIMES: A RETROSPECTIVE MATCHED COHORT STUDY
}

\author{
Hendrika J.M. Smelt ${ }^{1}$ \\ Sjaak Pouwels ${ }^{2}$ \\ M. Said ${ }^{2}$ \\ Kim A. Berghuis ${ }^{2}$ \\ Arjen-Kars Boer ${ }^{3}$ \\ Johannes F. Smulders ${ }^{2}$
}

Obes Surg. 2016 Dec; 26(12): 2873-2879 


\section{ABSTRACT}

\section{Background}

The incidence of vitamin B12 deficiency after bariatric surgery can range from $26-70 \%$. There is no consensus on optimal vitamin B12 supplementation in postbariatric patients. The objective of this study was to compare three different regimes.

\section{Methods}

In this retrospective matched cohort study, we included 63 patients with MMA levels $\geq$ $300 \mathrm{nmol} / \mathrm{L}$. Twenty-one received 5 intramuscular (IM) vitamin B12 injections including a loading dose, twenty one patients received 3 IM vitamin B12 injections without loading dose and twenty one patients received no IM vitamin B12 injections.

\section{Results}

The total patient population consisted of 14 males (22.2\%) and 49 women (77.8\%) with a mean current body mass index of $30.6 \pm 8.0 \mathrm{~kg} / \mathrm{m}^{2}$. There is no significant difference in vitamin B12 and MMA levels between 3 groups at baseline. There was a significant difference in followup vitamin B12 levels of the 5 IM injection regime compared to the 3 IM injection regime $(\mathrm{p}=0.02), 5 \mathrm{IM}$ injection regime compared to no regime $(\mathrm{p}=0.03)$. In the follow-up results there is also a significant decrease in MMA levels of the 5 IM injection regime compared to 3 IM injections ( $\mathrm{p}=0.02)$, 5 IM injection regime compared to no regime $(\mathrm{p}<0.001)$ and 3 IM injection regime compared to no regime $(\mathrm{p}<0.01)$.

\section{Conclusion}

In this study, the 3 IM injection schedule without a loading dose is not sufficient to treat a vitamin B12 deficiency. A 5 IM injection regime with loading dose recovered all vitamin B12 deficiencies biochemically. 


\subsection{INTRODUCTION}

The incidence of vitamin B12 deficiency after bariatric surgery can range from $26-70 \%$. A vitamin B12 deficiency occurs in the relatively early stage postoperative but most commonly years after the surgery when the large liver stores are slowly depleted (1). Vitamin B12 deficiency can cause pernicious/megaloblastic anemia, fatigue, light-headedness, numbness and paraesthesia (tingling or prickly feeling) in extremities, demyelination and axonal degeneration (especially of peripheral nerves, spinal cord and cerebrum), changes in mental status ranging from mild irritability and forgetfulness to severe dementia or frank psychosis, ataxia and change in reflexes (8-10).

To prevent a vitamin B12 deficiency after bariatric surgery, all patients have started to use an oral vitamin supplement with vitamin B12 daily from 2 weeks postoperative. In order to detect a vitamin B12 deficiency, patients are screened preoperatively and postoperatively. We have added the additional analysis of methylmalonic acid (MMA) with vitamin B12 levels below $300 \mathrm{pmol} / \mathrm{L}$ who quantifies functional intracellular shortage since June 2013 in our bariatric centre. In the first months we did not yet start intramuscular injections based on MMA, because there was no clear cut-off value established for MMA in bariatric patients. Nowadays, we started intramuscular vitamin B12 injections in bariatric patients with MMA levels $\geq 300 \mathrm{nmol} / \mathrm{L}$ since August 2013. The first period we used an injection schedule, which consisted of 5 injections including a loading dose. According to current literature there is no consensus about the right regime of intramuscular vitamin B12 injections and the necessity of a loading dose (1-6, 8). An internal investigation at our bariatric centre showed that MMA improved yet after a few injections. Since then, our injection regime has been modified to 3 injections without a loading dose (dosage of both regimes is included in the vitamin B12 treatment section).

However, it is not clear whether the shorter injection schedule without a loading dose is just as effective as a longer injection schedule with a loading dose after six months. In this study we wanted to compare a longer injection regime with loading dose with a shorter injection regime without loading dose. These 2 regimes will be compared with a control group who had no injection regime. We hypothesized that patients receiving 5 injections of vitamin B12 will have higher plasma vitamin B12 levels and lower MMA levels after six months compared to 3 injections and no injections. Secondly, a faster elevation in MMA levels after 3 injections is expected compared to treatment with 5 injections. 


\subsection{METHODS}

In this matched retrospective cohort study an analysis of vitamin B12 and MMA levels in bariatric patients was done. All patients underwent a sleeve gastrectomy or Roux-en-Y gastric bypass either as primary or a revisional procedure, from 2009 to 2015 in the Obesity Centre Catharina Hospital. Data of interest were patient characteristics, baseline levels vitamin B12 and MMA and follow-up vitamin B12 and MMA levels after 6 months.

We included 21 patients arbitrarily with complete data sets and MMA levels $\geq 300 \mathrm{nmol} / \mathrm{L}$ who received 5 intramuscular (IM) vitamin B12 injections including a loading dose, regardless of vitamin B12 levels. We also included 21 patients arbitrarily with complete data sets and MMA levels $\geq 300$ who received 3 IM vitamin B12 injections without loading dose, regardless of vitamin B12 levels. In order to determine whether MMA levels recover spontaneously over time, we have included a control group of 21 patients with MMA levels $\geq 300 \mathrm{nmol} / \mathrm{L}$. These patients did not receive IM vitamin B12 injections. Patients from the control group were selected arbitrarily from the database between June 2013 and August 2013.

All patients are advised to use a vitamin supplement daily. Initially, we recommended patients to use a high-dose weight loss surgery (WLS) supplement. However, these supplements are more expensive than over-the-counter supplements and some patients cannot afford these supplements. In case of side effects or intolerability for the supplements, an over-thecounter supplement was recommended $(1,7,8)$. Table 10.1 gives an overview of the dosage and percentage vitamin B12 of these supplements. In total, 16 patients $(25,4 \%)$ were using high-dose weight loss surgery (WLS) supplements, 43 patients $(68,3 \%)$ were using over-thecounter multivitamin supplements and 4 patients $(6,3 \%)$ did not use supplements.

TABLE 10.1. dosage of vitamin B12 in WLS supplements and over-the-counter supplements that are recommended in our centre

\begin{tabular}{|l|c|c|c|}
\hline & $\begin{array}{c}\text { Dosage tablets } \\
\text { per day }\end{array}$ & $\begin{array}{c}\text { Dosage vitamin B12 } \\
\text { in mcg per tablet }\end{array}$ & $\begin{array}{c}\text { Dosage vitamin B12 as } \\
\text { a percentage per tablet }\end{array}$ \\
\hline WLS sleeve & 1 & 10 & $400 \%$ \\
\hline WLS gastric bypass & 1 & 350 & $14000 \%$ \\
\hline $\begin{array}{l}\text { Over-the-counter } \\
\text { supplements sleeve }\end{array}$ & 1 & 2.5 & $100 \%$ \\
\hline $\begin{array}{l}\text { Over-the-counter } \\
\text { supplements gastric bypass }\end{array}$ & 2 & 2.5 & $100 \%$ \\
\hline
\end{tabular}




\section{VITAMIN B 12 SUPPLEMENTATION}

Vitamin B12 treatment consists of IM hydroxocobalamin injections, regardless type of regular vitamin supplements. Each injection contains 1000 micrograms of hydroxocobalamin.

Dosage of the old treatment regime: in total 5 injections with a loading dose of 1 injection every 2 weeks, during the first 8 weeks. Afterwards, one injection every three months.

Current treatment regime: in total 3 injections without loading dose. One injection in the first, second and the third month.

\section{MMA MEASUREMENT IN BLOOD SAMPLING}

MMA was measured in EDTA plasma using UPLC-MS/MS (Waters Xevo TQS). Methyl (D3)-malonic acid was used as internal standard. Mass transitions 116.9 - 73 and 119,9 76 were used to quantify these compounds, respectively. Ultrafiltration (Amicon Ultra 0.5 $\mathrm{mL}-30 \mathrm{~K}$, Millipore) was used as sample preparation and reverse phase chromatography (ACQUITY HSS T3, 100 x 2,1 mm, Waters) was used for separation on UPLC. Evaluation of MMA values in our hospital laboratory: MMA levels < $300 \mathrm{nmol} / \mathrm{L}$ are considered normal, MMA levels between $300-430 \mathrm{nmol} / \mathrm{L}$ are considered moderate deficient and MMA levels $>430 \mathrm{nmol} / \mathrm{L}$ are considered severe deficient by our institutional laboratory (7).

\section{STATISTICAL ANALYSIS}

Data were retrospectively collected, managed, and analysed. Continuous variables were presented as mean \pm standard deviation (SD). Categorical variables were presented as frequency with percentages. One-way ANOVA (with post-hoc Tukey-b test) was used to compare the baseline values and follow-up values of vitamin B12 and MMA.

A subanalysis of baseline vitamin B12 and MMA per type of surgery (sleeve gastrectomy, Roux-en-Y gastric bypass or revisional bariatric surgery) was performed also using the oneway ANOVA test. To analyse the differences in vitamin B12 and MMA levels, the delta was calculated (vitamin B12/MMA follow-up minus vitamin B12/MMA baseline). These data values were also analysed with the one-way ANOVA test.

In all tests, values of $\mathrm{p}<0.05$ were considered statistically significant. Statistical Package for Social Sciences (SPSS, Chicago, IL, USA Version 22.0) for Windows was used to prepare the database and for statistical analysis. 


\subsection{RESULTS}

The total patient population consisted of 14 males $(22.2 \%)$ and 49 women $(77.8 \%)$ with a mean current body mass index of $30.6 \pm 8.0 \mathrm{~kg} / \mathrm{m}^{2}$. Table 10.2 gives an overview of the other baseline characteristics.

TABLE 10.2. Baseline characteristics $(n=63)($ mean $\pm S D)$

\begin{tabular}{|c|c|c|c|c|}
\hline Different groups: & $\begin{array}{l}3 \text { IM injection } \\
\text { regime }\end{array}$ & $\begin{array}{l}5 \mathrm{IM} \text { injection } \\
\text { regime }\end{array}$ & $\begin{array}{l}\text { No injection } \\
\text { regime }\end{array}$ & P-value \\
\hline Age (years) & $39 \pm 11,9$ & $43,5 \pm 8,6$ & $44,7 \pm 9,0$ & $\mathrm{P}=0,990$ \\
\hline $\begin{array}{l}\text { Gender (n): } \\
\text { Male } \\
\text { Female }\end{array}$ & $\begin{array}{c}3 \\
18\end{array}$ & $\begin{array}{c}5 \\
16\end{array}$ & $\begin{array}{c}6 \\
15\end{array}$ & $P=0,616$ \\
\hline $\begin{array}{l}\text { Preoperative body mass index } \\
\left(\mathrm{kg} / \mathrm{m}^{2}\right)\end{array}$ & $43,8 \pm 7,6$ & $40,8 \pm 6,6$ & $43 \pm 6,9$ & $P=0,368$ \\
\hline $\begin{array}{l}\text { Current body mass index } \\
\left(\mathrm{kg} / \mathrm{m}^{2}\right)^{*}\end{array}$ & $32,7 \pm 12,4$ & $28,8 \pm 6,2$ & $30,5 \pm 5,5$ & $\mathrm{P}=0,347$ \\
\hline $\begin{array}{l}\text { Procedures (n): } \\
\text { Sleeve gastrectomy } \\
\text { Gastric bypass } \\
\text { Revision surgery }\end{array}$ & $\begin{array}{c}10 \\
8 \\
3\end{array}$ & $\begin{array}{l}9 \\
9 \\
3\end{array}$ & $\begin{array}{l}8 \\
8 \\
5\end{array}$ & $\mathrm{P}=0,905$ \\
\hline $\begin{array}{l}\text { Time postoperative }(\mathbf{n}) \text { : } \\
\leq 1 \text { year } \\
2 \text { years } \\
3 \text { years } \\
4 \text { years } \\
5 \text { years } \\
>5 \text { years }\end{array}$ & $\begin{array}{l}3 \\
9 \\
7 \\
1 \\
1 \\
0\end{array}$ & $\begin{array}{l}2 \\
6 \\
7 \\
3 \\
2 \\
1\end{array}$ & $\begin{array}{l}3 \\
9 \\
5 \\
2 \\
1 \\
1\end{array}$ & $\mathrm{P}=0,951$ \\
\hline
\end{tabular}

${ }^{*}$ Current body mass index measured at different time points postoperative.

Baseline levels and follow-up results showed a significant difference in vitamin B12 ( $<<0.05)$ and MMA ( $\mathrm{p}<0.05)$ after 3 IM injections. However, not all patients improved sufficiently. In 6 patients, MMA levels are not normalized below $300 \mathrm{nmol} / \mathrm{L}$ after $3 \mathrm{IM}$ injections. A significant difference was also seen in vitamin B12 ( $\mathrm{p}<0.05)$ and MMA $(\mathrm{P}<0.05)$ after 5 IM injections. MMA levels have been normalized below $300 \mathrm{nmol} / \mathrm{L}$ in all patients after $5 \mathrm{IM}$ injections. No significant difference was seen in vitamin B12 ( $\mathrm{p}=0.083)$ and a significant increase in MMA ( $\mathrm{p}$ $<0.05$ ) was seen without an IM injection regime (table 10.3). Eleven patients had MMA levels between 300 and $430 \mathrm{nmol} / \mathrm{L}$ (moderately deficient) and 5 patients had MMA levels above $430 \mathrm{nmol} / \mathrm{L}$ (severely deficient) at baseline without IM injection regime. In the follow up, all patients had MMA levels above $300 \mathrm{nmol} / \mathrm{L}$, of which 12 patients had MMA levels > 430 $\mathrm{nmol} / \mathrm{L}$ without IM injection regime. MMA levels cannot recover spontaneously over time. There is no significant difference in vitamin B12 and MMA levels between 3 groups 
at baseline. In the follow-up results there is a significant difference in vitamin B12 levels of the 5 IM injection regime compared to the 3 IM injection regime $(p=0.02)$, 5 IM injection regime compared to no regime $(\mathrm{p}=0.03)$ and 3 IM injection regime compared to no regime showed no significant difference $(\mathrm{p}=0.64)$. In the follow-up results there is also a significant decrease in MMA levels of the 5 IM injection regime compared to 3 IM injections ( $\mathrm{p}=0.02), 5 \mathrm{IM}$ injection regime compared to no regime ( $\mathrm{p}=<0.001)$ and 3 IM injection regime compared to no regime $(\mathrm{p}<0.01)$ (Table 10.4).

TABLE 10.3. biochemical effects of the different IM injection regimes and no regime (mean \pm SD).

\begin{tabular}{|l|l|c|c|c|}
\hline \multicolumn{2}{|c|}{} & Baseline & Follow-up & P-value \\
\hline \multirow{2}{*}{$\begin{array}{l}\text { 3 IM injection } \\
\text { regime }\end{array}$} & Vitamin B12 & $200,0 \pm 45,6$ & $332,9 \pm 296,5$ & $\mathrm{P}=0,046$ \\
\cline { 2 - 5 } & MMA & $455,8 \pm 168,1$ & $281,7 \pm 134,7$ & $\mathrm{P}=0,001$ \\
\hline \multirow{2}{*}{$\begin{array}{l}\text { IM injection } \\
\text { regime }\end{array}$} & Vitamin B12 & $200,5 \pm 36,0$ & $550,3 \pm 451,8$ & $\mathrm{P}=0,002$ \\
\cline { 2 - 5 } & MMA & $504,0 \pm 261,3$ & $181,1 \pm 64,5$ & $\mathrm{P}=0,000$ \\
\hline \multirow{2}{*}{$\begin{array}{l}\text { No regime } \\
\end{array}$} & Vitamin B12 & $226,2 \pm 34,6$ & $211,4 \pm 37,6$ & $\mathrm{P}=0,083$ \\
\cline { 2 - 5 } & MMA & $407,1 \pm 156,0$ & $514,3 \pm 235,9$ & $\mathrm{P}=0,004$ \\
\hline
\end{tabular}

IM: intramuscular, MMA: methylmalonic acid

TABLE 10.4. Biochemical effects of vitamin B12 and MMA levels between 3 groups at baseline and follow-up

\begin{tabular}{|l|c|c|c|c|}
\hline & 3 IM regime & 5 IM regime & No regime & P-value \\
\hline Baseline: & & & & \\
Vitamin B12 & $200,0 \pm 45,6$ & $200,5 \pm 36,0$ & $226,2 \pm 34,6$ & NS \\
MMA & $455,8 \pm 168,1$ & $504,0 \pm 261,3$ & $407,1 \pm 156,0$ & NS \\
\hline Follow-up: & & & & $*$ \\
Vitamin B12 & $332,9 \pm 296,5$ & $550,3 \pm 451,8$ & $211,4 \pm 37,6$ & $* *$ \\
MMA & $281,7 \pm 134,7$ & $181,1 \pm 64,5$ & $514,3 \pm 235,9$ & \\
\hline
\end{tabular}

IM: intramuscular, NS: not significant

*Values of the 5 IM injection regime compared to 3 IM injection regime $(p=0.02), 5$ IM injection regime compared no regime $(p=0.003), 3$ IM injection regime compared to no regime $(p=0.64)$

** Values of the 5 IM injection regime compared to 3 IM injection regime $(p=0.02), 5$ IM injection regime compared no regime $(\mathrm{p}<0.001), 3$ IM injection regime compared to no regime $(\mathrm{p}<0.01)$ 


\section{DELTA VITAMIN B 12 AND DELTA MMA}

Delta vitamin B12 and MMA was determined by the following calculation: "Follow up levels minus baseline values. Table 10.5 shows delta values of vitamin B12 and MMA levels. Vitamin B12 levels are rising faster and MMA levels give a faster decline after the 5 IM injection regime $(\mathrm{p}<0.01)$.

\section{SUBANALYSIS SLEEVE GASTRECTOMY VERSUS ROUX-EN-Y GASTRIC BYPASS VERSUS REVISIONAL SURGERY}

The surgical procedures consisted of 27 patients (42.9\%) with a sleeve gastrectomy, 25 patients (39.7\%) with a Roux-en-Y gastric bypass and 11 patients (17.5\%) with revisional surgery. In the sleeve gastrectomy group, a significant difference in follow-up results of MMA levels was seen in 5 IM injection regime compared to no regime $(p=0.001)$. In the Roux-en-Y gastric bypass group, a significant difference in followup results of MMA levels was seen in 3 IM injection regime compared to no regime $(\mathrm{p}=0.005)$ and 5 IM injection regime compared to no regime $(\mathrm{p}<0.001)$. In the revisional surgery group, a significant difference in baseline vitamin B12 levels was seen in 3 IM injection regime compared to no regime ( $p=0.016)$. A significant difference in follow-up MMA levels was seen in 3 and 5 IM injection regime compared to no regime ( $\mathrm{p}$ $<0.05)$. An overview of differences in baseline and follow-up results of vitamin and MMA levels per surgical procedure are showed in table 10.6.

TABLE 10.5. Delta values of vitamin B12 and MMA levels in the follow-up

\begin{tabular}{|l|c|c|c|c|}
\hline & 3 IM injection regime & 5 IM injection regime & No regime & P-value \\
\hline Delta vitamin B12 & $132,9 \pm 286,3$ & $349,8 \pm 454,6$ & $-14,8 \pm 37,1$ & $*$ \\
\hline Delta MMA & $-174,1 \pm 193,0$ & $-323,0 \pm 276,4$ & $107,2 \pm 150,8$ & $* *$ \\
\hline
\end{tabular}

*Values of 5 IM injection regime significant increase compared to no regimes $(p<0.01)$, values of 5 IM injection regime compared to 3 IM injection regime showed no significant difference ( $p=0.082)$, values of 3 IM injection regime compared to no regime showed no significant difference $(\mathrm{p}=0.388)$.

** Values of 5 IM injection regime showed a significant decrease compared to no regime $(\mathrm{p}<0.01)$, values of 5 IM injection regime compared to $3 \mathrm{IM}$ injection regime showed no significant difference $(\mathrm{p}=0.082)$, values of 3 IM injection regime compared to no regime showed a significant difference $(\mathrm{P}<0.01)$. 
TABLE 10.6. An overview of differences in baseline and follow-up results of vitamin B12 and MMA levels per surgical procedure.

\begin{tabular}{|c|c|c|c|c|c|}
\hline & & 3 IM & 5IM & No & P-value \\
\hline \multirow{4}{*}{$\begin{array}{l}\text { Sleeve } \\
\text { gastrectomy } \\
(\mathbf{n}=27)\end{array}$} & Baseline B12 & $198.0 \pm 31.6$ & $214.4 \pm 27.4$ & $232.5 \pm 37.3$ & NS \\
\hline & Baseline MMA & $463.7 \pm 152.7$ & $498.2 \pm 123.1$ & $354.5 \pm 54.8$ & NS \\
\hline & Follow-up B12 & $357.0 \pm 405.9$ & $665.6 \pm 507.6$ & $206.3 \pm 38.9$ & NS \\
\hline & Follow-up MMA & $329.3 \pm 165.7$ & $188.7 \pm 61.3$ & $496.9 \pm 177.6$ & * \\
\hline \multirow{4}{*}{$\begin{array}{l}\text { Gastric } \\
\text { bypass } \\
(n=25)\end{array}$} & Baseline B12 & $220.0 \pm 57.1$ & $191.1 \pm 44.6$ & $230.0 \pm 37.8$ & NS \\
\hline & Baseline MMA & $403.4 \pm 80.5$ & $546.6 \pm 382.9$ & $351.6 \pm 100.2$ & NS \\
\hline & Follow-up B12 & $337.5 \pm 184.8$ & $528.4 \pm 453.6$ & $220.0 \pm 46.6$ & NS \\
\hline & Follow-up MMA & $238.9 \pm 79.6$ & $183.2 \pm 77.1$ & $377.0 \pm 75.3$ & $* *$ \\
\hline \multirow{4}{*}{$\begin{array}{l}\text { Revision } \\
\text { surgery } \\
(\mathrm{n}=11)\end{array}$} & Baseline B12 & $153.1 \pm 5.8$ & $186.7 \pm 20.8$ & $210.0 \pm 24.5$ & $* * *$ \\
\hline & Baseline MMA & $569.3 \pm 352.5$ & $393.7 \pm 94.3$ & $579.8 \pm 223.5$ & NS \\
\hline & Follow-up B12 & $240.0 \pm 45.8$ & $270.0 \pm 90.0$ & $206.0 \pm 19.5$ & NS \\
\hline & Follow-up MMA & $237.0 \pm 118.5$ & $151.7 \pm 35.1$ & $761.8 \pm 313.5$ & $* * * *$ \\
\hline
\end{tabular}

* No regime compared to 5 IM injection regime $(\mathrm{p}=0.001), 3$ IM injection regime compared to no regime $(\mathrm{p}=$ $0.065), 3$ IM injection regime compared to 5 IM injection regime $(\mathrm{P}=0.132)$.

** No regime compared to 3 IM injection regime $(\mathrm{p}=0.005)$, no regime compared to 5 IM injection regime $(\mathrm{p}<$ $0.001)$, 3 IM injection regime compared to 5 IM injection regime $(\mathrm{p}=0.459)$.

*** No regime compared to 3 IM injection regime $(\mathrm{p}=0.016)$, No regime compared to 5 IM injection regime $(\mathrm{p}=$ $0.468), 3$ IM injection regime compared to 5 IM injection regime $(\mathrm{p}=1.00)$.

**** 3 IM injection regime compared to 5 IM injection regime $(\mathrm{p}=1.00)$, no regime compared to 5 IM injection regime $(\mathrm{p}=0.02)$, no regime compared to 3 IM injection regime $(\mathrm{p}=0.04)$.

\subsection{DISCUSSION}

This study showed that all patients with a vitamin B12 deficiency recovered well biochemically after 6 months follow up with the 5 IM injection regimen. After the 3 IM injection regimen some patients were still deficient after 6 months follow up. MMA levels cannot recover spontaneously over time without IM injection regime. In revision surgery procedures there was a significant difference in levels vitamin B12 and MMA at baseline in compared to sleeve gastrectomy and Roux-en-Y gastric bypass procedures. This is caused by the slowly depleted body storage of vitamin B12. After revisional surgery, the vitamin B12 storage may already be exhausted which possibly results in faster shortage after revision surgery. This may explain the differences at baseline levels in patients with revision surgery. MMA levels may be increased in renal insufficiency, dehydration and bacterial overgrowth. The intestinal flora produces propionic acid; which is a source of MMA. During antibiotic therapy MMA levels may be lower than normal. Patients suffering from these complaints were not included in this study (13). 
According to current literature there is no consensus about the right schedule of IM vitamin B12 injections and the necessity of a loading dose. Table 10.7 gives an overview of the recommended vitamin B12 treatment according to several studies. Only a few studies have a loading dose of vitamin B12 injections in their recommendation. However, our study shows that IM injection regime with loading dose gives better results, in compare to a shorter and monthly regime without loading dose.

\section{LIMITATIONS}

Some limitations need to be addressed. First, it is a small-size retrospective cohort study. Second, bariatric patients use different types of vitamin supplements that may have influence on vitamin B12 or MMA values in general. The body's storage of vitamin B12 is approximately $2000 \mathrm{mcg} /$ day in relation to the recommended daily requirement of $2,4 \mathrm{mcg} / \mathrm{day}$. While most vitamin B12 in normal adults is absorbed in the ileum in the presence of IF, approximately $1 \%$ of supplemented vitamin B12 will be absorbed passively (by diffusion) along the entire length of the (non-bypassed) intestine after bariatric surgery given a high-dose oral supplement (8). Rhode et al. (11) found that a dosage of $350-600 \mathrm{mcg} /$ day of oral vitamin B12 prevented vitamin B12 deficiency in $95 \%$ of patients and an oral dose of $500 \mathrm{mcg} /$ day was sufficient to overcome an existing deficiency as reported by Brolin et al. [12] in a similar study. About the recommended amount of vitamin B12 after a sleeve gastrectomy is no consensus in the current available literature. However, a lot of our patients use over-the-counter supplements with a dosage of vitamin B12 of 2,5 or $5 \mathrm{mcg} /$ day, for sleeve and gastric bypass, respectively. The body's storage will be depleted much faster in patients who using over-the-counter supplementation or no supplementation, in compare to patients who using high-dose WLS supplements.

In this study, a much faster and steeper rise in MMA levels was seen in patients who did not take their standard vitamin supplements regardless the injection regime. This is caused by the lack of a daily maintenance dose of vitamin B12 in combination with depleted liver stocks (1). Another important point is de absorption of the IM injections; about 10\% of the injected dosage is retained (10).

A difference in follow-up MMA levels was also observed between patients who used highdose WLS supplements and patients who used over-the-counter supplements. However, the groups of different kinds of supplements is too small to do statistical analysis. Besides that, these data are subjectively and it is unclear if patients take their supplements daily. Third, clinical aspects (complaints of vitamin B12 deficiency) are not included in this study because it is not clear which MMA values correlate with complaints. Despite these limitations, the results of the study have demonstrated that a shorter IM injection regime without loading dose is not sufficient for all patients in order to treat a vitamin B12 deficiency. 
TABLE 10.7. Various literature references and their recommended vitamin B12 treatment

\begin{tabular}{|c|c|}
\hline References & Conclusion vitamin B12 treatment \\
\hline Levinson et al., 2013 & Intramuscular vitamin B12 1000 mcg monthly or 3000 mcg every six moths \\
\hline Bordalo et al. 2011 & $500 \mathrm{mg} /$ day oral or $1000 \mathrm{mg} / \mathrm{mol} \mathrm{IM}$ or $3000 \mathrm{mg} / \mathrm{six}$ monthly IM \\
\hline Clements et al. 2006 & $1000 \mathrm{mcg}$ IM every 3 months or intranasal $1000 \mathrm{mcg}$ every week \\
\hline Jeder et al. 2010 & $\begin{array}{l}\text { Treatment first phase: } 350 \mathrm{mcg} / \mathrm{dag} \text { oral crystalline B12 } \\
\text { Treatment second phase: IM 1000-2000 mcg/2-3 months }\end{array}$ \\
\hline Bozkurt et al 2014 & $\begin{array}{l}350-600 \text { mcg oral vitamin B12 per day is effective in correcting deficiency in } 81 \text { to } \\
95 \% \text { of the patients and IM monthly are another option in patient who have trouble } \\
\text { adhering to daily oral supplement }\end{array}$ \\
\hline Stacy et al. 2010 & $\begin{array}{l}\text { Neurologic symptoms: IM } 1000 \mathrm{mcg} / \text { day for } 5 \text { days, followed by } 1000 \mathrm{mcg} \text { per month. } \\
\text { In patients who have had gastric bypass surgery: } 1000 \mathrm{mcg} \text { IM every } 3 \text { months }\end{array}$ \\
\hline Aills et al. 2008 & $\begin{array}{l}\text { Mild malabsorption: oral vitamin cyanocobalamin } 500-1000 \text { mcg or IM } 1000 \text { mcg daily } \\
\text { or every other day for } 1 \text { week, then weekly for } 4-8 \text { week, and then monthly for life } \\
\text { Severe malabsorption: IM } 1000 \mathrm{mcg} \text { daily or every other day for } 1 \text { week, then weekly } \\
\text { for } 4-8 \text { week, and then monthly for life }\end{array}$ \\
\hline Sally et al. 2013. & $\begin{array}{l}\text { Patients with severe deficiency should receive injections of } 1000 \text { mcg at least several } \\
\text { times per week for } 1 \text { to } 2 \text { weeks, then weekly until clear improvement is shown, } \\
\text { followed by monthly injections * }\end{array}$ \\
\hline
\end{tabular}

* This article is about a vitamin B12 deficiency in general. Not specifically for patients after bariatric surgery.

\subsection{CONCLUSION}

In this study, the 3 IM injection schedule without a loading dose is not sufficient to treat a vitamin B12 deficiency. A 5 IM injection regime with loading dose recovered all vitamin B12 deficiencies biochemically. MMAlevelscannotrecoverspontaneouslyovertimewithoutIMinjection regime. Compliance of intake of a standard vitamin supplementation and kind supplementation should be considered in decision-making to a certain injection regime. A randomised clinical trial is necessary to investigate different vitamin B12 supplementation regimes to define the most optimal one. Secondly, to examine potential placebo effects of IM vitamin B12 injections and cost effectiveness of the different IM injection regimes. 


\subsection{REFERENCES}

1. Levinson R, Silverman JB, Catella JG et al. Pharmacotherapy Prevention and Management of Nutritional Deficiencies Post Roux-en-Y Gastric Bypass. Obes Surg 2013; 23: 992-1000.

2. Bordalo LA, Teixeira TF, Bressan J, et al. Bariatric surgery: how and why to supplement. Rev Assoc Med Bras. 2011; 57(1):113-20.

3. Clements RH, Katasani VG, Palepu R, et al. Incidence of vitamin deficiency after laparoscopic Roux-en-Y gastric bypass in a university hospital setting. Am Surg. 2006;72:1196-1202; discussion 1203-1204.

4. Jeder D, Greemway FL, Kaplan LM, et al. Endocrine and nutritional management of the postbariatric surgery patient: and Endocrine Society Clinical Practice Guideline. J Clin Endocrinol Metab. 2010; 95(11):4823-43.

5. Bozkurt S. Complications in bariatric surgery. Eur J Endosc Laparosc Surg. 2014; 1(4):238-246.

6. Stacy A, Rudnicki MD. Prevention and treatment of peripheral neuropathy after bariatric surgery. Curr Treat Opt in Neurol. 2010; 12: 29-36.

7. HJM Smelt, JF Smulders, M. Said, SW Nienhuijs, AK Boer. Improving bariatric patient aftercare outcome by improved detection of a functional vitamin B12 deficiency. Obes Surg. 2015. DOI 10.1007/s11695-015-1952-8.

8. Aills L, Blankenship J, Buffington C, et al. ASMBS Allied health nutritional guidelines for surgical weight loss patient. Surgery for obesity and related diseases. 2008; 4: 73108.

9. Fava M, Borus JS, Alpert JE, Nierenberg AA, Rosenbaum JF, Bottiglieri T. Folate, vitamin $B_{12}$, and homocysteine in major depressive disorder. Am J Psychiatry. 1997; 154 :426-428.

10. Sally P, Stabler MD. Vitamin B12 deficiency - clinical practice. N Engl J Med. 2013; 368: 149-160.

11. Rhode BM, Tamim H, Gilfax MB. Treatment of vitamin B12 deficiency after gastric bypass surgery for severe obesity. Obes Surg 1995; 5:154-8.

12. Brolin RE, Gorman JH, Gorman RC, et al. Are vitamin B12 and folate deficiency clinically important after Roux-en-Y gastric bypass? J Gastrointest Surg 1998; 2:436-42.

13. Lindgren A. Elevated serum methylmalonic acid. How much comes from cobalamin deficiency and how much fasting comes from the kidneys? Scand J Clin Lab Invest. 2002; 62: 15-19. 



\title{
11. IMPROVED AND MORE EFFECTIVE ALGORITHMS TO SCREEN FOR NUTRIENT DEFICIENCIES AFTER BARIATRIC SURGERY
}

\author{
Inge Bazuin ${ }^{1}$ \\ Sjaak Pouwels ${ }^{2}$ \\ Saskia Houterman ${ }^{3}$ \\ Simon W. Nienhuijs ${ }^{2}$ \\ Johannes F. Smulders ${ }^{2}$ \\ Arjen-Kars Boer ${ }^{1}$
}

Eur J Clin Nutr. 2017 Feb;71(2):198-202 


\section{ABSTRACT}

\section{Background}

Most bariatric guidelines recommend frequent lab monitoring of patients to detect nutrient and vitamin deficiencies as early as possible. The aim of this study was to optimize the cost effectiveness of the nutrient panel, by developing an algorithm, which detects nutrient deficiencies at lower costs.

\section{Methods}

In this retrospective study, 2055 patients who had undergone Laparoscopic Roux-Y Gastric Bypass (LRYGB) and Laparoscopic Sleeve Gastrectomy (LSG) surgery at Catharina Hospital Eindhoven between January 2009 and December 2013. Perioperative biochemical measurements ( 7 days before and 127 days after surgery) and measurements $>549$ days before surgery were excluded. For analysis, the most recent pre- and postoperative measurements were selected for each biochemical parameter separately. Firstly step the amount of moderate and severe deficiencies were calculated. Secondly, we investigated whether each variable (vitamins $\mathrm{A}, \mathrm{B}_{1}, \mathrm{~B}_{6}, \mathrm{~B}_{12}, \mathrm{D}$, folate, ferritin, zinc and magnesium) could predict the presence of deficiency.

\section{Results}

In total, 561 (LRYGB) and 831 (LSG) patients had at least pre- and postoperative values of vitamin $A, B_{1}, B_{6}, B_{12}$, D, folate, ferritin, zinc or magnesium. The algorithm reduces vitamin D, $B_{12}, B_{6}, B_{1}$ and ferritin examinations by $15 \%, 11 \%, 28 \%, 28 \%$ and $38 \%$, respectively, without missing clinically relevant deficiencies. The corresponding potential cost savings was $14 \%$.

\section{Conclusion}

This study identified substantial cost savings in laboratory test for both LRYGB and LSG procedures. The potential cost reduction of $14 \%$ might even be increased to $42 \%$ when less frequent moderate deficiencies are not screened anymore, while $>99.0$ of moderate deficiencies will be detected. 


\subsection{INTRODUCTION}

Patients undergoing bariatric surgery are subjected to possible adverse physiological, nutritional and metabolic changes (1). In order to detect these changes as early as possible, most bariatric guidelines recommend frequent monitoring with extensive laboratory panel (2-6). However, the recommended laboratory panels differ in these guidelines. Furthermore, the guidelines sometimes disagree with respect to cut-off levels to detect deficiencies and generally do not specify the corresponding biochemical assays. For example the prevalence of folate deficiency, which can be established in plasma and erythrocytes, varies between 6 and $65 \%(4)$.

In our hospital the most performed bariatric surgical procedures are the Laparoscopic Roux-Y Gastric Bypass (LRYGB) and Laparoscopic Sleeve Gastrectomy (LSG). These patients are extensively screened for numerous biochemical parameters including the nutrients/vitamins $\mathrm{A}, \mathrm{B}_{1}, \mathrm{~B}_{6}, \mathrm{~B}_{12}, \mathrm{D}$, folate, ferritin, zinc and magnesium. Based on the Dutch national defined prices, the cost of this vitamin/nutrient panel is 72.60 euro. Since, the patient is monitored prior and up to 7 times after surgery the total costs of the follow-up period equals 580.80 euro. Since not all patients develop deficiencies and those who do, usually do not develop all deficiencies simultaneously, not all laboratory tests have clinical consequences. Reliable identification of the patients, which could be screened with less abundant laboratory panels, will result in a reduction of laboratory costs. Secondly, there is increasing governmental pressure to reduce healthcare costs and therefore this study was necessary.

The aim of this study was to optimize the cost effectiveness of the nutrient panel, by developing algorithms, which detect all nutrient deficiencies at lower costs. To develop these algorithms a retrospective analysis was performed on biochemical parameters of all LRYGB and LSG patients in our hospital in the past four years.

\subsection{METHODS}

\section{DATA SELECTION}

Patients who had undergone LRYGB or LSG surgery $(\mathrm{n}=2055)$ at Catharina Hospital Eindhoven between January 2009 and December 2013 with at least one preoperative and one postoperative laboratory examination were included in this retrospective study. Patients who had emergency or revision interventions were excluded. Perioperative biochemical measurements ( 7 days before and 127 days after surgery) and measurements $>549$ days before 
surgery were excluded. For analysis, the most recent pre- and postoperative measurements were selected for each biochemical parameter separately. In total 561 LRYGB and 831 LGS patients were included. The Institutional Review Board approved this study and for this type of study formal patient consent is not required.

\section{ALGORITHM DEVELOPMENT}

All of our patients were screened for vitamins $A, B_{1}, B_{6}, B_{12}, D$, folate, ferritin, zinc and magnesium. We were interested whether the costs of lab testing in our center could be reduced. Therefore, the first step was to calculate the amount of moderate and severe deficiencies of the above mentioned (Table 11 S1).

TABLE 11 S1. Overview of the percentages of patients moderately and severe deficient prior and after surgery in LRYGB and LSG group

\begin{tabular}{|c|c|c|c|c|c|}
\hline Variable & $\begin{array}{l}\text { Type of } \\
\text { surgery }\end{array}$ & $\begin{array}{l}\text { \% Moderate } \\
\text { deficient } \\
\text { preoperative }\end{array}$ & $\begin{array}{c}\text { \% Severe } \\
\text { deficient } \\
\text { preoperative }\end{array}$ & $\begin{array}{c}\text { \% Moderate } \\
\text { deficient } \\
\text { postoperative }\end{array}$ & $\begin{array}{c}\text { \% Severe } \\
\text { deficient } \\
\text { postoperative }\end{array}$ \\
\hline \multirow{2}{*}{ Ferritin } & LRYGB & 20 & 5.2 & 16 & 3.9 \\
\hline & LSG & 15 & 4.8 & 12 & 4.7 \\
\hline \multirow{2}{*}{ Folate } & LRYGB & 20 & 1.8 & 7.9 & 1.2 \\
\hline & LSG & 21 & 2.3 & 14 & 2.2 \\
\hline \multirow{2}{*}{ Magnesium } & LRYGB & 3 & 0 & 3.5 & 0 \\
\hline & LSG & 2.4 & 0 & 3.9 & 0 \\
\hline \multirow{2}{*}{ Vitamin A } & LRYGB & 0 & 0 & 0.5 & 0 \\
\hline & LSG & 0 & 0 & 0 & 0 \\
\hline \multirow{2}{*}{ Vitamin $B_{1}$} & LRYGB & 0.2 & 0.2 & 0.6 & 0.2 \\
\hline & LSG & 0.3 & 0.2 & 1.2 & 0.3 \\
\hline \multirow{2}{*}{ Vitamin $\mathrm{B}_{6}$} & LRYGB & 1.4 & 0 & 0.2 & 0 \\
\hline & LSG & 0.9 & 0 & 1.2 & 0 \\
\hline \multirow{2}{*}{ Vitamin $B_{12}$} & LRYGB & 7.1 & 7.1 & 3.6 & 3.6 \\
\hline & LSG & 6.3 & 6.3 & 5.3 & 5.3 \\
\hline \multirow{2}{*}{ Vitamin D } & LRYGB & 70 & 21 & 22 & 3.1 \\
\hline & LSG & 76 & 25 & 23 & 3.2 \\
\hline \multirow{2}{*}{ Zinc $<10.7$} & LRYGB & 22 & 0 & 35 & 0 \\
\hline & LSG & 21 & 0 & 29 & 0.2 \\
\hline \multirow{2}{*}{ Zinc $<8.4$} & LRYGB & 0.5 & 0 & 3 & 0 \\
\hline & LSG & 1 & 0 & 2.2 & 0.2 \\
\hline
\end{tabular}

Abbreviations: LRYGB = Laparoscopic Roux-Y Gastric Bypass, LSG = Laparoscopic Sleeve Gastrectomy 
Secondly, we investigated whether each variable (vitamins $A, B_{1}, B_{6}, B_{12}, D$, folate, ferritin, zinc and magnesium) could predict the presence of deficiency. This was done by calculating combination of deficiencies. For example in how many times is there a combined vitamin $\mathrm{A}$ and vitamin $B_{12}$ deficiency? Or how many times are folate and vitamin $B_{12}$ deficiencies present before and after surgery? These combinations were made for vitamins $\mathrm{A}, \mathrm{B}_{1}, \mathrm{~B}_{6}, \mathrm{~B}_{12}, \mathrm{D}$, folate, ferritin, zinc and magnesium.

\section{SUPPLEMENTATION REGIME}

Patients were screened for vitamins $\mathrm{A}, \mathrm{B}_{1}, \mathrm{~B}_{6}, \mathrm{~B}_{12}$, D, folate, ferritin, zinc and magnesium preoperative and 6,12,18, 24, 36, 48, 60 months postoperative. Table 11.1 gives an overview of the definitions of deficiencies of the screened vitamins and minerals.

Most studies use one set of cut-off values to define nutrient deficiencies, which usually equals the method-specific lower reference limit (LRL). However, in literature also specific supplementation limits have been defined. Most supplementation limits are lower than LRL. In this study two sets of cut-off values were used to define nutrient deficiencies. Measurements below LRL are defined as moderate deficiencies and measurements below the supplementation limit as severe deficiencies. Only for ferritin and vitamin D the recommended supplementation limit is higher than our LRL.

After bariatric surgery, patients were instructed to take 200\% (LRYGB) or 100\% (LSG) of the daily recommend dose of vitamins and minerals. Specific nutrient deficiencies were additional supplemented. When iron was $<10 \mathrm{nmol} / \mathrm{L}$ and ferritin was $<20 \mu \mathrm{g} / \mathrm{L}$ ferrofumarate $(200$ $\mathrm{mg}$ ) was administered twice a day, in combination with vitamin C (500 mg) once a day. Vitamin D $<50 \mathrm{nmol} / \mathrm{L}$ was supplemented with $1 \mathrm{~mL}$ colecalciferol $(50,000 \mathrm{IU} / \mathrm{mL})$ weekly during the first 4-6 weeks and subsequently monthly. Folate $<6.0 \mathrm{nmol} / \mathrm{L}$ was corrected with folic acid ( $5 \mathrm{mg} /$ day) for 3 months. Vitamin $\mathrm{B}_{12}<145 \mathrm{pmol} / \mathrm{L}$ was supplemented with hydroxycobalamin injections of $500 \mu \mathrm{g} / \mathrm{mL}$ once per two weeks in the first 2 months and once per 3 months afterwards. Vitamin $B_{1}<70 \mathrm{nmol} / \mathrm{L}$ was supplemented with $50-100 \mathrm{mg} /$ day thiamine, for 3 months. There was no standardized supplementation protocol for vitamin $\mathrm{A}, \mathrm{B}_{6}$, magnesium and zinc. 
TABLE 11.1. Definitions of moderate and severe deficiencies

\begin{tabular}{|l|c|c|c|c|c|}
\hline & \multicolumn{2}{|c|}{ Moderate deficiency } & \multicolumn{2}{c|}{ Severe deficiency } & Unit \\
\hline Ferritin & $<30$ & $(8-10)$ & $\begin{array}{c}<\underline{13}(\square) \\
<\underline{23}(\square)\end{array}$ & $(4,9,11-14)$ & $\mu \mathrm{g} / \mathrm{L}$ \\
\hline Folate (serum) & $<\underline{10.4}$ & $(10,15)$ & $<6.7$ & $(8,9,11,13,14,16,17)$ & $\mathrm{nmol} / \mathrm{L}$ \\
\hline Magnesium (plasma) & $<\underline{0.70}$ & $(8,13,17-19)$ & $<0.50$ & $(8,17)$ & $\mathrm{mmol} / \mathrm{L}$ \\
\hline Vitamin A (retinol) & $<\underline{0.70}$ & $(8,17)$ & $<0.35$ & $(8,17)$ & $\mu \mathrm{mol} / \mathrm{L}$ \\
\hline Vitamin $\mathrm{B}_{1}(\mathrm{TPP})$ & $<\underline{70}$ & $(8)$ & $<55$ & $(20)$ & $\mathrm{nmol} / \mathrm{L}$ \\
\hline Vitamin $\mathrm{B}_{6}(\mathrm{P} 5 \mathrm{P})$ & $<\underline{35}$ & $\#$ & $<20$ & $(4,8,16,17,20)$ & $\mathrm{nmol} / \mathrm{L}$ \\
\hline Vitamin B $(\mathrm{serum})$ & $<\underline{145}$ & $\begin{array}{c}(4,8,11,13,14, \\
16,17,21)\end{array}$ & $<145$ & $(4,8,11,13,14,16$, & $\mathrm{pmol} / \mathrm{L}$ \\
\hline Vitamin D (25-OH) & $<50$ & $(4,8,11,13,14$, & $<25$ & $(8,10,11,13,17)$ & $\mathrm{nmol} / \mathrm{L}$ \\
\hline Zinc (plasma) & $<10.7$ & $(8-10,13,17,19)$ & $<5.0$ & $(17)$ & $\mu \mathrm{mol} / \mathrm{L}$ \\
\hline
\end{tabular}

Abbreviations: $\mu \mathrm{g} / \mathrm{L}=$ microgramme per litre, $\mathrm{nmol} / \mathrm{L}=$ nanomole per litre, $\mathrm{mmol} / \mathrm{L}=$ millimole per litre, $\mathrm{pmol} / \mathrm{L}$ $=$ picomole per litre, $\mu \mathrm{mol} / \mathrm{L}=$ micromole per litre

The underlined values are the method specific reference values. ${ }^{*}$ The lower reference values for vitamin $\mathrm{D}$ and zinc are $17 \mathrm{nmol} / \mathrm{L}$ and $8.4 \mu \mathrm{mol} / \mathrm{L}$, respectively. However, the cut-off levels 10.7 (zinc) and $50 \mathrm{nmol} / \mathrm{L}$ (vitamin D) are the most frequently mentioned levels in literature. ": Operating manual of vitamin $\mathrm{B}_{6}$, chromosystems. TPP: thiamin pyrophosphate, P5P: pyrodoxal-5-phosphate.

\section{BIOCHEMICAL ASSAY}

The utilised laboratory for our study is certified by the Dutch Association of Clinical Chemistry Labs (CCKL, registration number R0125). Independent clinical chemists did the biochemical analysis of the vitamins and minerals. Vitamin A was determined as retinol in serum with a UPLC-TUV (Waters ${ }^{\circledR}$ ) instrument using Repice ${ }^{\circledR}$ reagents. Vitamin $\mathrm{B}_{1}$ (thiamin pyrophosphate) and vitamin $\mathrm{B}_{6}$ (pyridoxal-5-phosphate) were determined in EDTA-whole blood with chromosystems ${ }^{\circledast}$ reagents on a UPLC - FLR (Waters ${ }^{\circledR}$ ) device. Vitamin D (25-hydroxy vitamin D) was determined in serum by an immunometric competition assay on Liason $^{\circledast}$ using Diasorin ${ }^{\circledast}$ reagents. Vitamin $\mathrm{B}_{12}$ (cobalamin) serum, folate serum and ferritin heparin plasma were analyzed by immunometric assays on the cobas E-module Roche ${ }^{\circledast}$. Magnesium was determined in heparin plasma by a colorimetric endpoint assay on the cobas C-module Roche ${ }^{\circledast}$. Zinc was determined in plasma on an atomic absorption spectrometer $\left(\right.$ PerkinElmer $\left.{ }^{\circledR}\right)$. Reference values are shown in table 11.1. 


\section{STATISTICAL ANALYSIS}

The pre and postoperative prevalence were analyzed with McNemar test. A p-value $<0.05$ was considered as significant. IBM Statistical Package for the Social Sciences 22 (SPSS) was used for statistical analysis. The development of the algorithms was based on prevalence of deficiencies before and after bariatric surgery. For the determination of potential cost reduction and sensitivity, all laboratory values were included of LRYGB and LSG patients and secondly sensitivity analysis was performed used a random effects model. Sensitivity was determined by dividing the percentages of missing deficiencies by all known deficiencies of the biochemical parameter. The potential cost savings were calculated by using Dutch national prices.

\subsection{RESULTS}

The most common deficiencies were vitamin $\mathrm{D}$, zinc, ferritin, folate and vitamin $\mathrm{B}_{12}$ (Figure 11.1). Follow-up ranged from 3 to 84 months postoperative for each nutrient and vitamin determined, with a mean of 32 months. For the development of the algorithms it was important to have an indication of the pre- and postoperative prevalences of deficiencies and the moment a deficiency was established for the first time. Vitamin D, ferritin and folate deficiencies decreased significant after both bariatric surgeries $(\mathrm{p}<0.05)$. Vitamin $\mathrm{B}_{12}$ deficiencies decreased significantly after LRYGB $(p=0.017)$ and not after LSG $(p=0.461)$. Using the cut-off value of $10.7 \mu \mathrm{mol} / \mathrm{L}$ zinc is the second frequent deficiency and increased after both surgeries $(p<0.05)$. However, when the lower zinc reference value of 8.4 $\mu \mathrm{mol} / \mathrm{L}$ was used to define a moderate zinc deficiency, the postoperative prevalences of the zinc deficiency was only 3\% and 2\% for LRYGB and LSG, respectively. In figure 11.1 the percentages of newly developed deficiencies are shown. Unexpectedly, most deficiencies were already present before surgery. For example, more than $82 \%$ of all postoperative vitamin D deficiencies and $64 \%$ of ferritin deficiencies were diagnosed prior to surgery. Magnesium, vitamin $B_{1}, B_{6}$ and $A$ deficiencies were rare and the pre and postoperative prevalence of these deficiencies were not significantly different ( $p>0.05$ ). Moreover, no severe deficiencies were found for these biochemical parameters.

We designed an algorithm for efficient screening for vitamin $\mathrm{D}$ deficiencies based on the observation that 1$)$ the majority $(82-90 \%)$ of vitamin $\mathrm{D}$ deficiencies are present prior to surgery and 2) that most newly diagnosed post-surgery deficiencies have a preoperative vitamin D sampled in the spring/summer with concentrations between 50 and $70 \mathrm{nmol} / \mathrm{L}$. $(27,28)$ Therefore, we used the cut-off levels of 50 and $70 \mathrm{nmol} / \mathrm{L}$ (for measurements in the periods October - March and April - September, respectively) to determine whether 
subsequent post-surgery vitamin D measurements were necessary or not (figure 11.2). This algorithm resulted in a $15 \%$ reduction of vitamin D measurements at the expense that only 98.5\% of the patients were correctly categorized between deficient and not-deficient. Except for two patients, all missed deficiencies $(n=37)$ were moderate deficiencies.

Since folate $\left(\mathrm{B}_{9}\right)$ is the most frequent $\mathrm{B}$-vitamin deficiency and is a useful marker for monitoring compliance of vitamin supplementation (7), we designed algorithms for vitamin $\mathrm{B}_{1}, \mathrm{~B}_{6}$ and $\mathrm{B}_{12}$ based on the folate concentration in the same order. For the vitamin $\mathrm{B}_{1}$ algorithm we also include the haemoglobin level to identify the clinically relevant vitamin $\mathrm{B}_{1}$ deficiencies, which are not captured by the folate concentration alone. (2-7) Using these algorithms $11-28 \%$ unnecessary B-vitamin measurements could be avoided (see figure 11.2).

Since iron deficiency is reflected in lowered ferritin, MCV and haemoglobin levels, we designed an algorithm based on these parameters to decide whether ferritin testing is necessary or not. When ferritin is only measured when the preoperative ferritin is $<75 \mu \mathrm{g} / \mathrm{L}$ and when $\mathrm{MCV}<80 \mathrm{fL}$ or haemoglobin $<8.0 \mathrm{mmol} / \mathrm{L}, 38 \%$ of the ferritin measurements could be avoided without missing clinically relevant iron deficiencies.

By using the algorithms in combination with the exclusion of nutrients from the standard panel (e.g. vitamin $A, B_{1}$ and $B_{6}$ ), which have only moderate corresponding deficiencies and, moreover, (very) low prevalences, the potential cost savings are $23-42 \%$ at the expense of 0.5 $-1.0 \%$ missed deficiencies. When only severe deficiencies should be identified the potential cost savings run up to $60 \%$ (Table 11.2) 


\section{A: LRYGB}

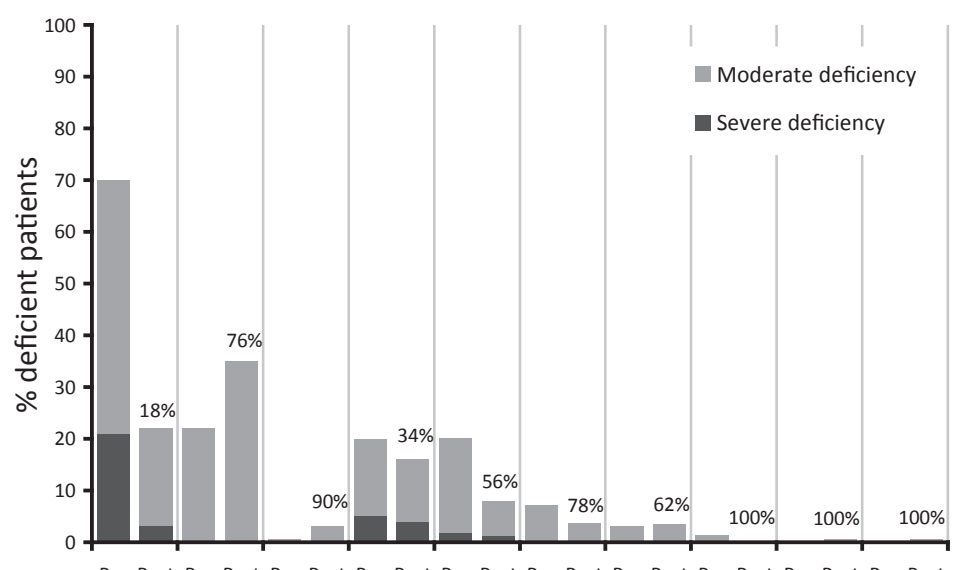

Pre Post Pre Post Pre Post Pre Post Pre Post Pre Post Pre Post Pre Post Pre Post Pre Post

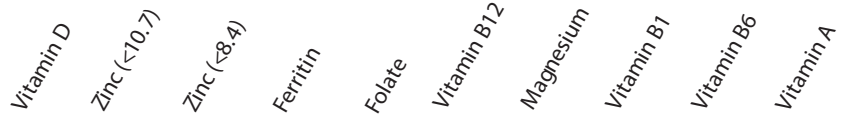

\section{B: LSG}
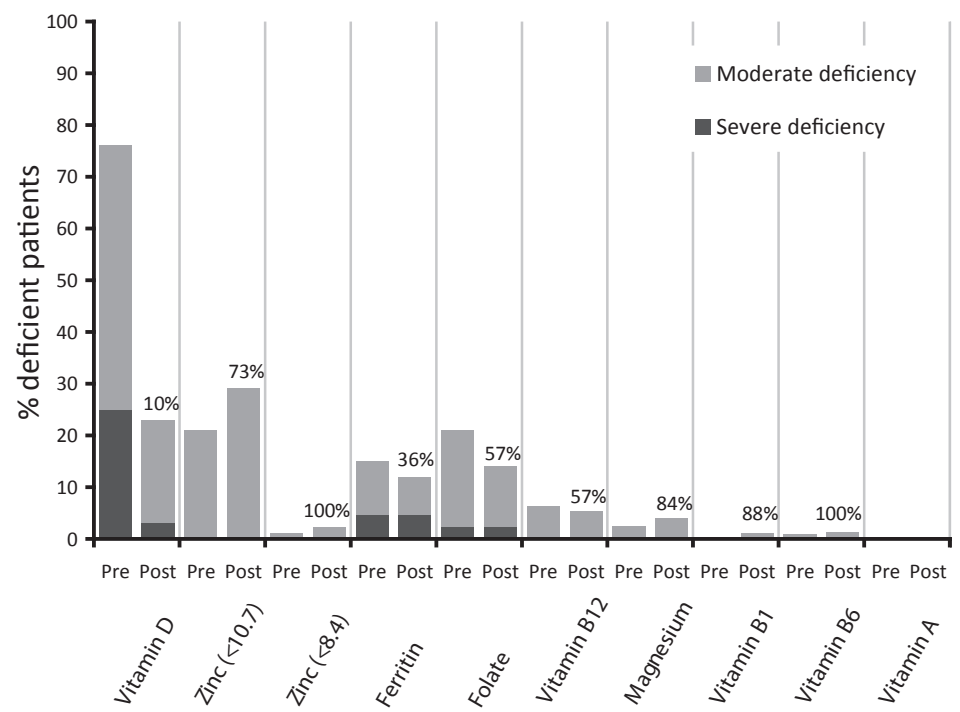

FIGURE 11.1. Pre- and postoperative prevalence of nutrient deficiencies undergoing A) LRYGB or B) LSG.

The bars indicate the total number of moderate deficiencies. The dark grey part represents the severe deficiencies. Mean follow-up ranged from 3 to 84 months. Above the bars the percentage of newly developed deficiencies post surgery are shown. 
A

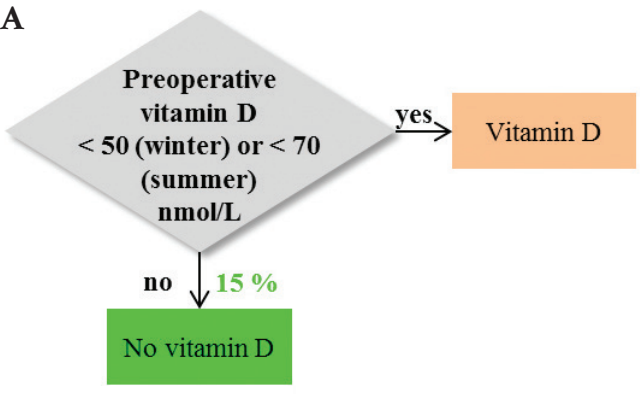

C

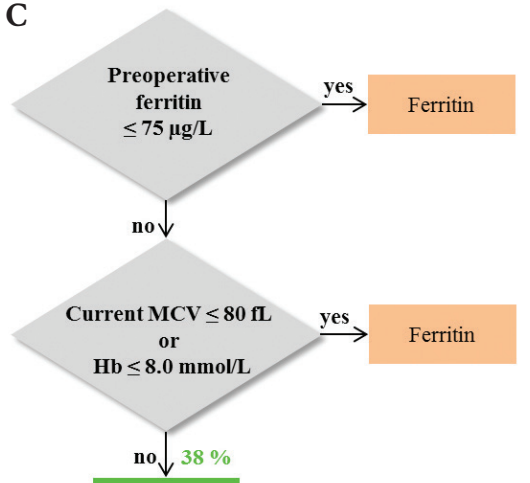

No Ferritin

D

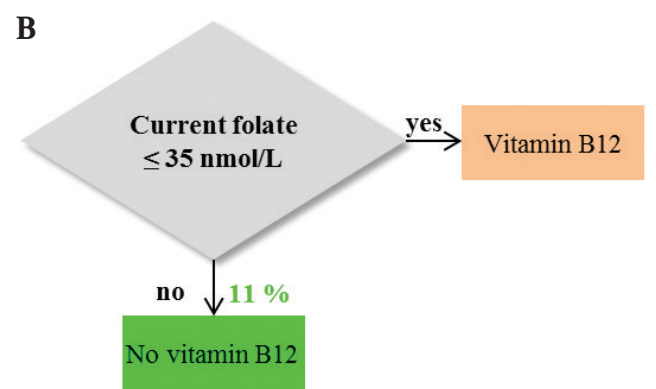

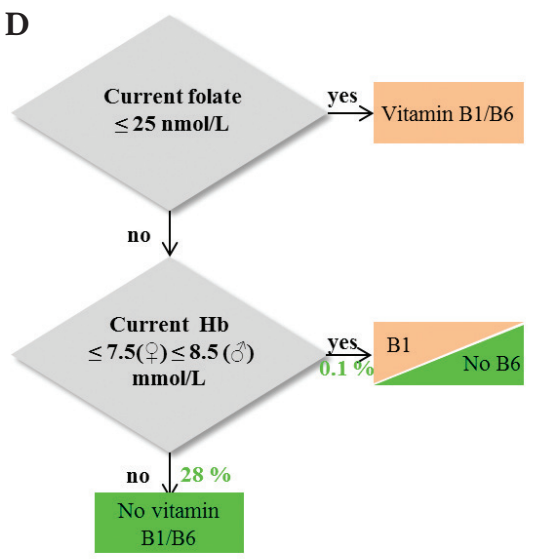

FIGURE 11.2. Algorithms for assessing A) vitamin $D, B$ ) vitamin $B_{12^{\prime}} C$ ) ferritin and D) vitamin $B_{1} / B_{6}$ laboratory tests.

The criteria in the decision windows determine whether the corresponding parameter should be measured or not. The green percentage indicates the reduction of measurements. See text for addition information. Vitamin D (50 $\mathrm{nmol} / \mathrm{L}$ equals $20 \mathrm{ng} / \mathrm{ml}$ ), folate ( 35 and $25 \mathrm{nmol} / \mathrm{L}$ equals 15 and $11 \mathrm{ng} / \mathrm{mL}$, respectively), ferritin ( $70 \mu \mathrm{g} / \mathrm{L}$ equals $70 \mathrm{ng} / \mathrm{L}$ ) Hemoglobin (7.5, 8.0 and 8.5 equals $12,12.9$ and $13.7 \mathrm{~g} / \mathrm{dL}$, respectively.)

$\mathrm{Hb}$ : hemogloblin, Winter: October - March, summer: April - September 
TABLE 11.2. Potential costs savings using the algorithm for A) LRYGB and B) LSG

\begin{tabular}{|c|c|c|c|c|c|c|}
\hline \multirow{3}{*}{$\begin{array}{l}\text { A: LRYGB } \\
\text { Nutrient panel }\end{array}$} & \multicolumn{3}{|c|}{ Moderate } & \multicolumn{3}{|c|}{ Severe } \\
\hline & \multirow{2}{*}{$\frac{\text { Cost }(€)}{508}$} & \multicolumn{2}{|c|}{ Cost savings } & \multirow{2}{*}{$\frac{\text { Cost }(\boldsymbol{€})}{508}$} & \multicolumn{2}{|c|}{ Cost savings } \\
\hline & & - & & & - & \\
\hline Algorithm & 437 & $14 \%$ & & 437 & $14 \%$ & \\
\hline $99.9 \%$ accuracy & 437 & $14 \%$ & & 250 & $51 \%$ & $\mathrm{M}, \mathrm{A}, \mathrm{B} 6, \mathrm{Z}$ \\
\hline $99.5 \%$ accuracy & 388 & $23 \%$ & B6 & 201 & $60 \%$ & $\mathrm{M}, \mathrm{A}, \mathrm{B} 1, \mathrm{~B} 6, \mathrm{Z}$ \\
\hline $99.0 \%$ accuracy & 293 & $42 \%$ & $\mathrm{~A}, \mathrm{~B} 1, \mathrm{~B} 6$ & 201 & $60 \%$ & $\mathrm{M}, \mathrm{A}, \mathrm{B} 1, \mathrm{~B} 6, \mathrm{Z}$ \\
\hline
\end{tabular}

\begin{tabular}{|c|c|c|c|c|c|c|}
\hline \multirow{3}{*}{$\begin{array}{l}\text { B: LSG } \\
\text { Nutrient panel }\end{array}$} & \multicolumn{3}{|c|}{ Moderate } & \multicolumn{3}{|c|}{ Severe } \\
\hline & \multirow{2}{*}{$\begin{array}{c}\text { Cost }(€) \\
508\end{array}$} & \multicolumn{2}{|c|}{ Cost savings } & \multirow{2}{*}{$\begin{array}{c}\text { Cost }(€) \\
508\end{array}$} & \multicolumn{2}{|c|}{ Cost savings } \\
\hline & & - & & & - & \\
\hline Algorithm & 437 & $14 \%$ & & 437 & $14 \%$ & \\
\hline $99.9 \%$ accuracy & 391 & $23 \%$ & A & 319 & $37 \%$ & $\mathrm{M}, \mathrm{A}, \mathrm{B} 6$ \\
\hline $99.5 \%$ accuracy & 391 & $23 \%$ & A & 201 & $60 \%$ & $\mathrm{M}, \mathrm{A}, \mathrm{B} 1, \mathrm{~B} 6, \mathrm{Z}$ \\
\hline $99.0 \%$ accuracy & 391 & $23 \%$ & A & 201 & $60 \%$ & $\mathrm{M}, \mathrm{A}, \mathrm{B} 1,6 \mathrm{~B}, \mathrm{Z}$ \\
\hline
\end{tabular}

Costs are calculated by multiplying the Dutch national defined prices with the vitamins in the 7 phlebotomies postsurgery. The superscript letters indicate which nutrient were excluded from the labpanel, where ${ }^{\mathrm{M}=}$ magnesium, ${ }^{\mathrm{A}=}$ vitamin $\mathrm{A},{ }_{1}^{\mathrm{B}}=$ vitamin $\mathrm{B}_{1},{ }_{6}^{\mathrm{B}}=$ vitamin $\mathrm{B}_{6}$ and ${ }^{\mathrm{Z}}=$ zinc.

Abbreviations: LRYGB = Laparoscopic Roux-en-Y Gastric Bypass, LSG = Laparoscopic Sleeve Gastrectomy

\subsection{DISCUSSION}

This study shows that substantial cost savings are possible in the detection of nutrient deficiencies under a standard supplementation regime after LRYGB and LSG surgery.

In our hospital we introduced one screening panel for both LRYGB and LSG patients, which consist of vitamin $\mathrm{A}, \mathrm{B}_{1}, \mathrm{~B}_{6}, \mathrm{~B}_{12}, \mathrm{D}$, folate, ferritine, and magnesium and zinc (2-6). Although, some guidelines also recommend screening for vitamin $\mathrm{E}$ and $\mathrm{K}$ deficiencies, we did not include these vitamins in the panel, because these deficiencies occur more often after biliopancreatic diversion/ duodenal switch (BPD/ BPD-DS) and are rare after LRYGB and LSG (22). Moreover, vitamin K levels were not useful for patients using anticoagulants for any comorbidity.

In order to correctly recognize nutrient deficiencies, well-established cut-off values are required. However, there is no consensus in bariatric literature. Different cut-off values are used to define deficiencies, frequently without mentioning the matrix (e.g. serum/ plasma or whole blood) or method used $(23,24)$. The matrix is important since major changes might 
occur when sampling in different matrices. For example, vitamin $\mathrm{B}_{1} / \mathrm{B}_{6}$, can both be determined in whole blood or in plasma, where blood values are much higher since approximately $80 \%$ of vitamin $B_{1} / B_{6}$ is in the erythrocytes (8). Another example is zinc, where serum zinc levels are generally 5 to $15 \%$ higher than plasma values due to osmotic changes in the erythrocytes by the anticoagulant used (17). Specification of the analytical method used, is also important, since nutrients can be measured in different forms. For example vitamin $\mathrm{B}_{1}$ can be determined as thiamine and as thiamine-pyrophosphate and vitamin A can be measured as retinol, retinal and retinoic acid, each with different reference and cut-off values. Furthermore, commercial nutrient methods are poorly standardized, resulting in differences up to $30 \%$ according to the Dutch external quality schemes, even when type of method and matrix are identical. Usage of lower reference values as cut-off value is not ideal to identify deficient patients. Firstly, by definition $2.5 \%$ of the healthy persons have values below the LRL and will be unnecessarily supplemented if the LRL is used as cut-off limit. Secondly, poor evidence exists that patients with mild or moderate deficiencies clinically benefit from nutrient supplementation. However, hard cut-off values for nutrient supplementation have been described (table 11.1). To avoid the discussion which cut-off level should be used, we have shown our data using two sets of cut-off values for both moderate and severe deficiencies.

The development of the algorithms was based on the prevalence of deficiencies. The prevalences of deficiencies in LRYGB and LSG patient groups were comparable (Table 11 S1). Therefore the algorithms were developed regardless the type of surgery.

All algorithms, except for the vitamin $\mathrm{D}$ algorithm, result in a major decrease in nutrient measurements without missing clinically relevant deficiencies. The percentage of missed vitamin D deficiencies, however, is low and most deficiencies are mild. However, in nonbariatric settings, multiple studies have shown that patients benefit from vitamin $\mathrm{D}$ supplementation when plasma levels are below $50 \mathrm{nmol} / \mathrm{L}$. Therefore, one might argue that the algorithm is not clinically useful. On the other hand, $98.5 \%$ of the bariatric patients were correctly categorized between deficient and not-deficient, while in the general European population the prevalence of severe vitamin D deficiency $(<25 \mathrm{nmol} / \mathrm{L})$ is already $2-30 \%$ (25). Furthermore, due to the seasonal changes of vitamin $\mathrm{D}$, in the winter period about half of healthy population have vitamin $\mathrm{D}$ levels $<50 \mathrm{nmol} / \mathrm{L}$ while supplementation is not prescribed. $(25,27,28)$ The study performed by Peterson et al., who found comparable results, supports our findings. (26) They found significant malnutrition prior to bariatric surgery in a cohort of 58 patients, especially Vitamin D (92.6\% of their cohort was deficient) and Iron (36.2 to $56.9 \%)$. (26)

We have not designed an algorithm for zinc, magnesium and vitamin A, since no severe deficiencies are present in our study. Furthermore, none of the patients have magnesium and 
vitamin A levels below the recommended supplementation limit $(8,17)$. On the other hand, moderate zinc deficiencies are present depending on the cut-off limits used. According to ASMBS nutrient guideline of 2008 zinc should be supplemented below $10.7 \mu \mathrm{mol} / \mathrm{L}$, while the LRL of zinc is $9.1 \mu \mathrm{mol} / \mathrm{L}$. The LRL of our method is $8.4 \mu \mathrm{mol} / \mathrm{L}$. Using the cut-off value of $10.7 \mu \mathrm{mol} / \mathrm{L}$ will result in unnecessary treatment of $10 \%$ of healthy persons, while in the general population persons with similar levels are not supplemented. A limit of $10.7 \mu \mathrm{mol} / \mathrm{L}$ seems therefore inappropriate.

When the prevalence of zinc deficiencies was calculated with the LRL of $8.4 \mu \mathrm{mol} / \mathrm{L}$, the postoperative zinc deficiency is only 3\% for LRYGB and 2\% for LSG patients. This is not significantly different from the general population. Severe deficiencies under $5 \mu \mathrm{mol} / \mathrm{L}$ are extremely rare.

Our postulated algorithms result in reduction of laboratory costs of $14 \%$ without missing clinically relevant deficiencies, except for vitamin D. Because the prevalences of moderate deficiencies of vitamin $A, B_{1}$ and $B_{6}$ are relatively low, one might exclude these biochemical parameters from the standard nutrient panel. This will result in an additional cost reduction of $28 \%$. Furthermore, the maximum of $1 \%$ missed deficiencies should be placed in perspective, since the prevalence of deficiencies is very high in the morbid obese population in general and the preoperative group in particular. $(13,14)$ The relative high preoperative prevalence of deficiencies and for some biochemical parameters the relative low percentage of newly developed deficiencies after surgery, suggest that the preoperative patient should be supplemented with higher doses of multivitamins.

\section{LIMITATIONS}

Of course, retrospective analyses have their limitations. For example, missing values might occur when labpanels were manually ordered. Furthermore, the adherence of multivitamin supplemention was not monitored. Also the dietary nutrient and vitamin intake might differ between patients after LRYGB and LSG and might result in under- and/or over-reportation of deficiencies in these populations. However in a larger sample retrospective analysis, we were not able to adjust for this confounder. Possibly the cost savings might be higher when only non-compliant persons are screened for deficiencies. Since we have no information about compliance, only prospective studies might determine the true accuracy and reduction in costs.

Secondly, problems arise because of inconsistency in the definition of the deficiency according to several guidelines and lack of consensus of the appropriate way to measure the level of a particular nutrient. Thirdly, this is a single site study and there might be differences in outcomes when our algorithm is used in other laboratories. Our algorithm is only applicable 
for primary LRYGB and LSG patients (our included study population) and might not be suitable for other bariatric (revisional) procedures. Also we have to consider that our algorithm might not be appropriate for patients $>7$ years after bariatric surgery. Further validation studies need to determine this.

\subsection{CONCLUSION}

This study shows that there are substantial cost savings possible in laboratory tests in LRYGB and LSG patients. With our algorithms, $14 \%$ of costs might be saved, without missing relevant deficiency. The cost savings can be up to $42 \%$, when biochemical parameters with a low prevalence of nutrient deficiencies are removed from the nutrient panel. In light of our findings we have to take into account that this study has variability in follow-up and this is a single center/laboratory study. 


\subsection{REFERENCES}

1 Shah M, Simha V, Garg A. Long-term impact of bariatric surgery on body weight, comorbidities, and nutritional status. Journal of Clinical Endocrinology \& Metabolism. 2006;91(11):4223-31.

2 Fried M, Yumuk V, Oppert J, Scopinaro N, Torres AJ, Weiner R, et al. Interdisciplinary european guidelines on metabolic and bariatric surgery. Obesity facts. 2013;6(5):449-68.

3 Heber D, Greenway FL, Kaplan LM, Livingston E, Salvador J, Still C. Endocrine and nutritional management of the post-bariatric surgery patient: An endocrine society clinical practice guideline. Journal of Clinical Endocrinology \& Metabolism. 2010;95(11):4823-43.

4 Aills L, Blankenship J, Buffington C, Furtado M, Parrott J. ASMBS allied health nutritional guidelines for the surgical weight loss patient. Surgery for Obesity and Related Diseases. 2008;4(5):S73-S108.

5 Mechanick JI, Youdim A, Jones DB, Garvey WT, Hurley DL, McMahon MM, et al. Clinical practice guidelines for the perioperative nutritional, metabolic, and nonsurgical support of the bariatric surgery patient-2013 update: Cosponsored by american association of clinical endocrinologists, the obesity society, and american society for metabolic \& bariatric surgery*. Obesity. 2013;21(S1):S1-S27.

6 Fried M, Hainer V, Basdevant A, Buchwald H, Deitel M, Finer N, et al. Interdisciplinary european guidelines on surgery of severe obesity. Obes Facts. 2008;1(1):52-9.

7 Brolin RE, Gorman RC, Milgrim LM, Kenler HA. Multivitamin prophylaxis in prevention of post-gastric bypass vitamin and mineral deficiencies. Int J Obes. 1991 Oct;15(10):661-7.

8 Williamson MA, Snyder LM. Wallach's interpretation of diagnostic tests. Lippincott Williams \& Wilkins; 2012.

9 Gasteyger C, Suter M, Gaillard RC, Giusti V. Nutritional deficiencies after roux-en-Y gastric bypass for morbid obesity often cannot be prevented by standard multivitamin supplementation. Am J Clin Nutr. 2008 May;87(5):1128-33.

10 Lefebvre P, Letois F, Sultan A, Nocca D, Mura T, Galtier F. Nutrient deficiencies in patients with obesity considering bariatric surgery: A cross-sectional study. Surgery for Obesity and Related Diseases. 2013.

11 Toh SY, Zarshenas N, Jorgensen J. Prevalence of nutrient deficiencies in bariatric patients. Nutrition. 2009;25(11):1150-6.

12 Gesquiere I, Lannoo M, Augustijns P, Matthys C, Van der Schueren B, Foulon V. Iron deficiency after roux-en-Y gastric bypass: Insufficient iron absorption from oral iron supplements. Obesity Surg. 2014;24(1):56-61.

13 Ernst B, Thurnheer M, Schmid SM, Schultes B. Evidence for the necessity to systematically assess micronutrient status prior to bariatric surgery. Obesity Surg. 2009;19(1):66-73.

14 Blume CA, Boni CC, Casagrande DS, Rizzolli J, Padoin AV, Mottin CC. Nutritional profile of patients before and after roux-en-Y gastric bypass: 3-year follow-up. Obesity Surg. 2012;22(11):1676-85. 
15 Pfeiffer CM, Johnson CL, Jain RB, Yetley EA, Picciano MF, Rader JI, et al. Trends in blood folate and vitamin B-12 concentrations in the united states, 1988 2004. Am J Clin Nutr. 2007 Sep;86(3):718-27.

16 Damms-Machado A, Friedrich A, Kramer KM, Stingel K, Meile T, Küper MA, et al. Preand postoperative nutritional deficiencies in obese patients undergoing laparoscopic sleeve gastrectomy. Obesity Surg. 2012;22(6):881-9.

17 Burtis CA, Ashwood ER, Bruns DE. Tietz textbook of clinical chemistry and molecular diagnostics. Elsevier Health Sciences; 2012.

18 Wu AH. Tietz clinical guide to laboratory tests. Saunders/Elsevier St. Louis; 2006.

19 Dolan K, Hatzifotis M, Newbury L, Lowe N, Fielding G. A clinical and nutritional comparison of biliopancreatic diversion with and without duodenal switch. Ann Surg. 2004 Jul;240(1):51-6.

20 Aasheim ET, Bjorkman S, Sovik TT, Engstrom M, Hanvold SE, Mala T, et al. Vitamin status after bariatric surgery: A randomized study of gastric bypass and duodenal switch. Am J Clin Nutr. 2009 Jul;90(1):15-22.

21 Schweiger C, Weiss R, Berry E, Keidar A. Nutritional deficiencies in bariatric surgery candidates. Obesity Surg. 2010;20(2):193-7.

22 Poitou Bernert C, Ciangura C, Coupaye M, Czernichow S, Bouillot J, Basdevant A. Nutritional deficiency after gastric bypass: Diagnosis, prevention and treatment. Diabetes Metab. 2007;33(1):13-24.

23 Madan AK, Orth WS, Tichansky DS, Ternovits CA. Vitamin and trace mineral levels after laparoscopic gastric bypass. Obesity Surg. 2006;16(5):603-6.

24 Flancbaum L, Belsley S, Drake V, Colarusso T, Tayler E. Preoperative nutritional status of patients undergoing roux-en-Y gastric bypass for morbid obesity. Journal of Gastrointestinal Surgery. 2006;10(7):1033-7.

25 Lips P. Vitamin D status and nutrition in Europe and Asia. J Steroid Biochem Mol Biol. 2007;103(3):620-5.

26 Peterson LA, Cheskin LJ, Furtado M, Papas K, Schweitzer MA, Magnuson TH, Steele KE. Malnutrition in bariatric surgery candidates: Multiple micronutrient deficiencies prior to surgery. Obes Surg. 2015, aug 22 (epub ahead of print)

27 Kasahara AK, Singh RJ, Noymer A. Vitamin D (25OHD) Serum Seasonality in the United States. PLoS One 2013;8: e65785. 28 Kroll MH, Bi C, Garber CC, Kaufman HW, Liu D, Caston-Balderrama A, Zhang K, Clarke N, Xie M, Reitz RE, Suffin SC, Holick MF. Temporal relationship between vitamin D status and parathyroid hormone in the United States. PLoS One 2015;10: e0118108. 

1. (1)

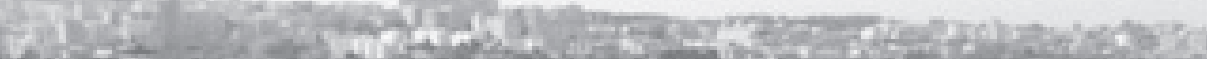

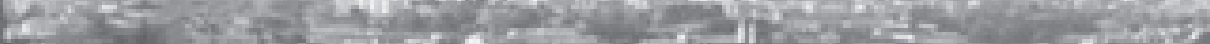

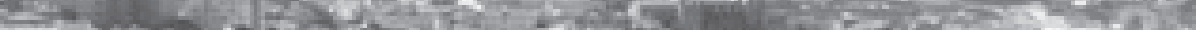

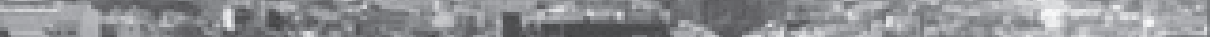

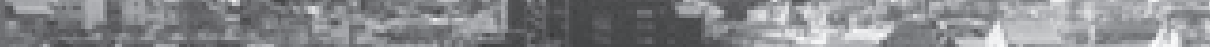

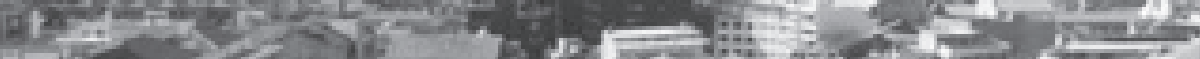
If

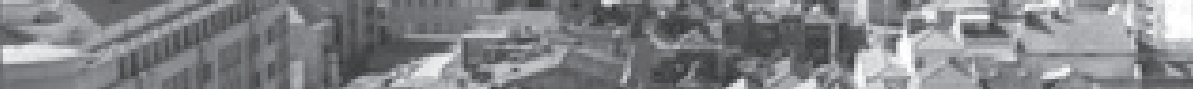

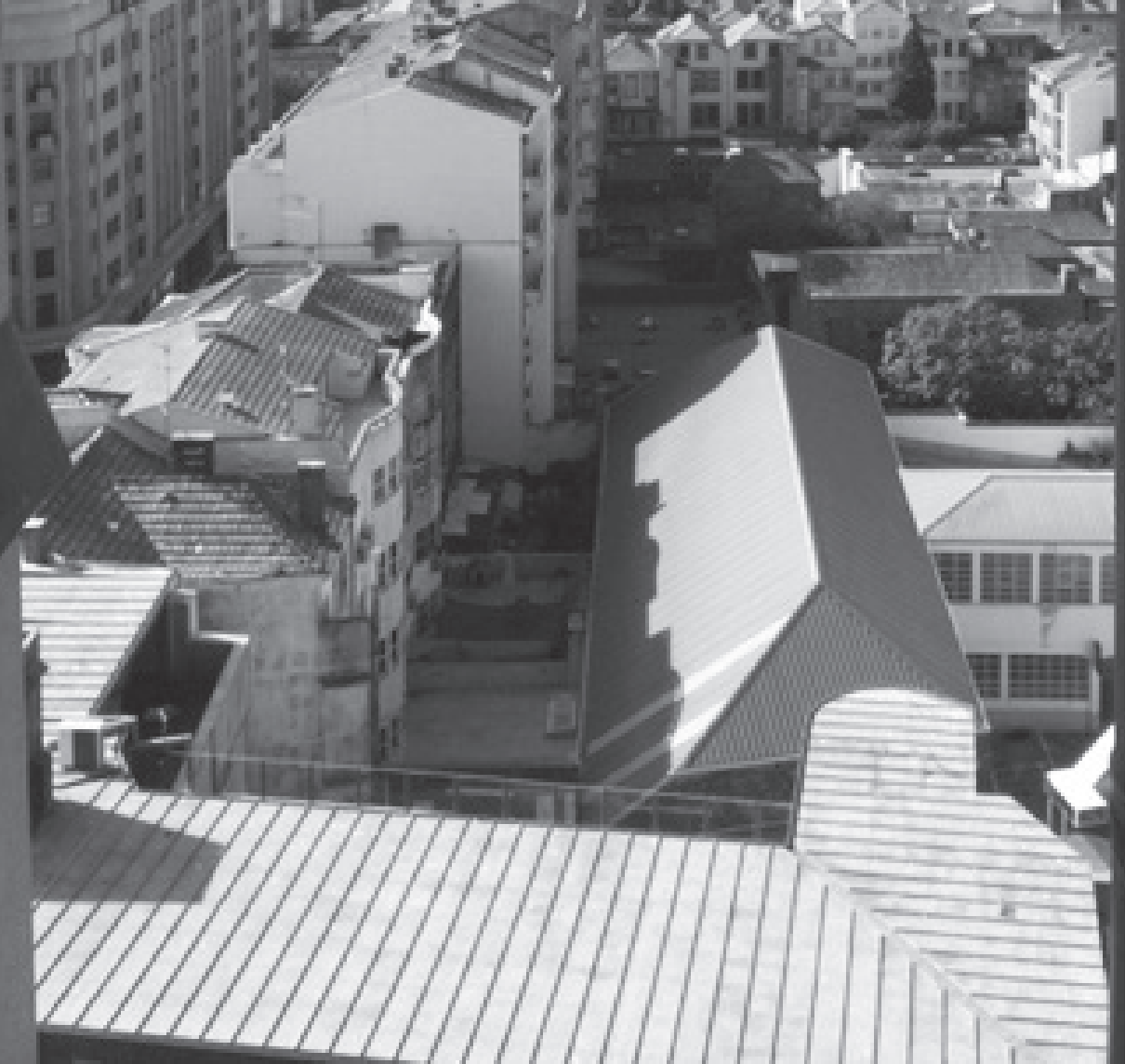




\title{
12. TIME TO GLYCEMIC CONTROL: AN OBSERVATIONAL STUDY OF 3 DIFFERENT OPERATIONS
}

\author{
Alper Celik ${ }^{1}$, \\ Sjaak Pouwels ${ }^{2}$, \\ Fatih Can Karaca ${ }^{1}$, \\ Eylem Çağıltay ${ }^{3}$, \\ Surendra Ugale ${ }^{4}$, \\ İlker Etikan ${ }^{5}$, \\ Deniz Büyükbozkırlı ${ }^{1}$, \\ Yunus Emre Kılıç ${ }^{1}$
}

Obes Surg. 2017 Mar;27(3):694-702 


\section{ABSTRACT}

\section{Background}

Medical treatment fails to provide adequate control for many obese patients with type 2 diabetes mellitus (T2DM). A comparative observational study of bariatric procedures was performed to investigate the time at which patients achieve glycemic control within the first 30 postoperative days following sleeve gastrectomy (SG), mini-gastric bypass (MGB), and diverted sleeve gastrectomy with ileal transposition (DSIT).

\section{Methods}

Included patients had a body mass index $(\mathrm{BMI}) \geq 30 \mathrm{~kg} / \mathrm{m}^{2}$; T2DM for $\geq 3$ years, $\mathrm{HbA}_{1 \mathrm{C}}>7 \%$ for $\geq 3$ months, and no significant weight change ( $>3 \%)$ within the prior 3 months. Surgical procedures performed were SG $(n=49)$, MGB $(n=93)$, and DSIT $(n=109)$. The primary endpoint was the day within the first postoperative month on which mean fasting capillary glucose levels reached $<126 \mathrm{mg} / \mathrm{dL}$. Multivariate logistic regression analysis was used to identify predictors of glycemic control.

\section{Results}

The cohort included 251 patients with a mean BMI of $36.04 \pm 5.76 \mathrm{~kg} / \mathrm{m}^{2}$; age, $52.84 \pm 8.52$ years; T2DM duration, $13.09 \pm 7.54$ years; $\mathrm{HbA}_{1 \mathrm{C}}, 8.82 \pm 1.58 \%$. On the morning of surgery, mean fasting plasma glucose was $177.63 \pm 51.3 \mathrm{mg} / \mathrm{dL}$; on day $30,131.35 \pm 28.7 \mathrm{mg} / \mathrm{dL}$ (p<0.05). Mean fasting plasma glucose of $<126 \mathrm{mg} / \mathrm{dL}$ was reached in the DSIT group (124.36 \pm 20.21 $\mathrm{mg} / \mathrm{dL})$ on day 29 , and in the MGB group $(123.61 \pm 22.51 \mathrm{mg} / \mathrm{dL})$, on day 30 . The SG group did not achieve the target mean capillary glucose level within postoperative 30 days.

\section{Conclusion}

During the first postoperative month, glycemic control $(<126 \mathrm{mg} / \mathrm{dL})$ was achieved following DSIT and MGB, but not SG. Preoperative BMI and postprandial C-peptide levels were independent predictors of early glycemic control following DSIT. 


\subsection{INTRODUCTION}

Obesity and type 2 diabetes mellitus (T2DM) have become global problems during recent decades. Unfortunately, medical treatment fails to provide adequate glycemic control in obese diabetics (1-5). A growing body of literature shows that bariatric surgery achieves favorable metabolic outcomes, is associated with a reduction in cardiovascular events and mortality, and can provide effective treatment for T2DM in obese patients (6-16). Bariatric surgical outcomes include improved glycemic control, reduction of required medications, fewer complications, remission of T2DM, and prevention of diabetes in non-diabetic patients (17). However, T2DM is a heterogeneous and dynamic disease. Despite evidence that glycemic control is achieved rapidly following bariatric surgery (sometimes within days), its relationship to a low-calorie perioperative diet and to weight loss is not linear $(18,19)$.

Although diabetes remission after bariatric surgery is a well-recognized phenomenon, evidence reporting the time frame of remission is sparse, as is the best means of managing diabetes in patients who do not experience rapid remission. Briatore and Scopinaro and colleagues and Fenske et al have reported on treatments with insulin analogues that produced a long-lasting effect for up to one year (11-13). This effect may be explained by the period of pancreatic regeneration stimulated in the early postoperative period. A healthy glucose environment is beneficial for beta cell function not only in the short, but also in the long term.

Our group investigated the glycemic regulation patterns of type 2 diabetics following 3 different surgical procedures. Postoperative glucose levels were measured twice a day by patients treated by either sleeve gastrectomy (SG), mini-gastric bypass (MGB), or diverted sleeve gastrectomy with ileal transposition (DSIT). Short-term diabetic outcomes could be examined in a mainly restrictive (SG) and mainly malabsorptive (MGB) procedure, as well as in a novel surgical technique with combined effects but without significant malabsorption (DSIT). DSIT is a relatively new metabolic surgical procedure that has been shown to be effective for diabetes control in lower BMIs, with favorable nutritional outcomes. It is associated with high rates of diabetes remission and a marked decrease in the use of medications after one year (14).

We report findings of a retrospective comparative observational study to investigate the time required for patients to achieve glycemic control after three different surgical options within the first 30 postoperative days. The association of glycemic control with patterns of weight loss in these groups was also analyzed. 


\subsection{METHODS}

\section{STUDY DESIGN}

The independent ethics committee of our Institutional Review Board approved this prospective comparative cohort study (14/10/2011, no. 08/2011). The study took place between November 2011 and March 2015. This study was designed in accordance with our Center of Excellence in Bariatric and Metabolic Surgery guidelines and followed principles of the Declaration of Helsinki and Good Clinical Practices. Patients were informed about the purpose of the study and gave informed consent prior to surgery. The study was conducted according to the Strengthening the Reporting of Observational studies in Epidemiology (STROBE) statement. Diagnosis of T2DM was established based on the criteria of the American Diabetes Association (15). Study design was based on the retrospective analysis of prospectively collected data from patient follow-up charts.

\section{INCLUSION CRITERIA}

Inclusion criteria for surgery were: duration of T2DM under continuous medical treatment for at least 3 years, $\mathrm{HbA}_{1 \mathrm{C}}>7 \%$ for more than 3 months, weight stability (defined as no significant change [ $>3 \%$ ] within the prior 3 months), and body mass index (BMI) $\geq 30 \mathrm{~kg} /$ $\mathrm{m}^{2}$. Specific inclusion criterion for the present study was a fasting glucose level $<200 \mathrm{mg} / \mathrm{dL}$ during the hospital stay, before discharge. All patients received oral and written information about the surgical options, and decisions were made with the medical team. CONSORT diagram that demonstrates patient flow is presented in Figure 12.1.

\section{EXCLUSION CRITERIA}

Patients were excluded from surgical treatment if they had a fasting C-peptide level $<0.5 \mathrm{ng} /$ $\mathrm{mL}$ or anti-GAD positivity. Other exclusion criteria included: previous major gastrointestinal surgery, pregnancy, intolerance to anesthesia, severe eating problems, and/or the use of psychopharmacological drugs for eating problems. Patients requiring oral anti-diabetic and/ or insulin treatment during discharge were excluded from the present study.

\section{OUTCOME MEASURES}

The numbers of patients in SG, MGB, and DSIT groups were 49 (19.5\%), 93 (37.1\%), and 109 (43.4\%), respectively. Age, $\mathrm{BMI}, \mathrm{HbA}_{1 \mathrm{C}}$, fructosamine, one-month weight loss, fasting and one-hour postprandial plasma, C-peptide, and total insulin levels of all patients were measured and recorded. For this purpose, all patients when presenting for the first time underwent a one-hour mixed-meal tolerance test. High-density lipoprototein (HDL), low- 
density lipoprotein (LDL), triglycerides (TG), comorbidities, and T2DM duration were also examined. During their stay in the hospital, blood glucose levels were measured in the morning (before breakfast) and night (before dinner), and weight was measured during the fasting state in the morning and recorded. Weight was measured by the nursing staff (not self-reported). Normoglycemia was defined as fasting plasma glucose $\leq 126 \mathrm{mg} / \mathrm{dL}$ without medication.

\section{PATIENT CARE}

Patients received the same preoperative preparation, including two days of liquid diet and hospitalization the day before surgery. Discharged patients were given forms for postoperative follow-up (Figure 12.4). Their glucometer calibrations were controlled before discharge. Patients were asked to record their blood glucose levels, blood pressure levels and weight during the fasting state in the morning, and also in the evening, and to bring the forms with them to the first month control visit.

\section{STATISTICAL ANALYSIS}

Statistical analyses were performed using SPSS software (IBM SPSS Statistics, ver. 19, Somers, NY, USA). Data for continuous variables are presented as means \pm standard deviations, and categorical variables as frequencies or percentages. Differences in the baseline values of surgery types were analyzed using analysis of variance (ANOVA) followed by Tukey's HSD test, as appropriate. Multivariate logistic regression analysis was used to determine the independent relationships between surgery type and changes in weight and plasma glucose levels. P values $<0.05$ were considered statistically significant. 


\section{Enrollment}

Assessed for eligibility $(n=943)$

Excluded ( $n=146)$

- Not meeting inclusion criteria $(n=41)$

- Unavailable data $(n=32)$

- Other reasons $(n=73)$

\section{Allocation}

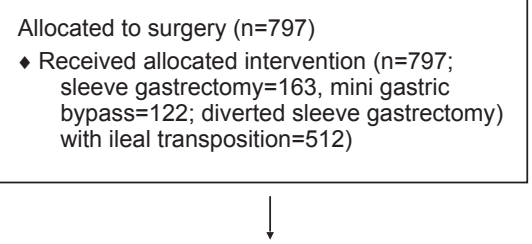

\section{Follow-Up}

- Not meeting inclusion criteria $(n=41)$

- Other reasons $(n=505)$

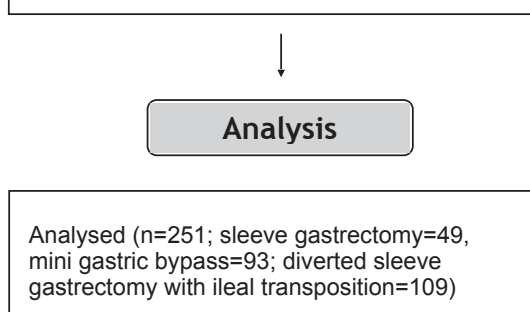

FIGURE 12.1: CONSORT flow diagram for recruitment of patients in this study

\subsection{RESULTS}

The study groups consisted of 251 patients (111 females, 140 males) with a mean age of 52.84 \pm 8.52 years and a mean preoperative BMI of $36.04 \pm 5.76 \mathrm{~kg} / \mathrm{m}^{2}$. All patients had poorly controlled diabetes as shown by a mean $\mathrm{HbA}_{1 \mathrm{C}}$ of $8.82 \pm 1.58 \%$, and a mean fructosamine level of $324.2 \pm 69.95(\mathrm{mmol} / \mathrm{L})$. Mean diabetic duration was $13.1 \pm 7.54$ years.

All patients had T2DM, demonstrated by mean fasting C-peptide of $2.69 \pm 1.09 \mathrm{ng} / \mathrm{ml}$ and mean postprandial C-peptide of $3.94 \pm 1.84 \mathrm{ng} / \mathrm{ml}$. Mean fasting and postprandial plasma 
insulin levels were $19.12 \pm 23.3$, and $36 \pm 34.16$, respectively. Hypertension, as defined by values exceeding 140/90 mm Hg, was noted in 145 individuals (57.8\%). Mean levels of total cholesterol, HDL cholesterol, LDL cholesterol, and triglyceride were 212.16 \pm 47.82 , 40.58 $\pm 13.14,132.32 \pm 36.49$, and $223.44 \pm 180.87 \mathrm{mg} / \mathrm{dL}$, respectively. Mean weight loss during the first month was $12.23 \pm 4.74$ kilograms. Table 12.1 provides an overview of baseline characteristics.

TABLE 12.1. Baseline parameters of surgery groups ( ${ }^{a}$ and ${ }^{b}$ refer to statistical significance)

\begin{tabular}{|c|c|c|c|c|}
\hline & $\begin{array}{c}\text { DSIT } \\
(\text { mean } \pm \text { SD })\end{array}$ & $\begin{array}{c}\text { SG } \\
(\text { mean } \pm \text { SD })\end{array}$ & $\begin{array}{c}\text { MGB } \\
(\text { mean } \pm S D)\end{array}$ & P-value \\
\hline Age (years) & $51.89 \pm 8.4$ & $54.2 \pm 8.2$ & $53.23 \pm 8.78$ & 0.247 \\
\hline BMI & $33.98 \pm 4.81^{\mathrm{a}}$ & $39.32 \pm 6.87^{\mathrm{b}}$ & $36.72 \pm 5.2^{\mathrm{c}}$ & $<0.001$ \\
\hline First month weight loss (kg) & $12.17 \pm 3.9$ & $13.22 \pm 7.39$ & $11.56 \pm 3.35$ & 0.249 \\
\hline $\mathrm{HbA}_{1 \mathrm{C}}(\%)$ & $9.09 \pm 1.73^{\mathrm{a}}$ & $8.24 \pm 1.71^{\mathrm{b}}$ & $8.82 \pm 1,23^{\mathrm{ab}}$ & 0.007 \\
\hline Fructosamine $(\mu \mathrm{mol} / \mathrm{l})$ & $343.69 \pm 63.13^{\mathrm{a}}$ & $300.71 \pm 80.9^{b}$ & $313,72 \pm 65.94^{\mathrm{b}}$ & $<0.001$ \\
\hline Fasting C-peptide (ng/ml) & $2.49 \pm 0.8^{\mathrm{a}}$ & $3.35 \pm 1.51^{\mathrm{b}}$ & $2.57 \pm 0.99^{\mathrm{a}}$ & $<0.001$ \\
\hline $\begin{array}{l}\text { Postprandial } \\
\text { C peptide (ng/ml) }\end{array}$ & $3.57 \pm 1.13^{\mathrm{a}}$ & $5.39 \pm 2.81^{\mathrm{b}}$ & $3.61 \pm 1.48^{\mathrm{a}}$ & $<0.001$ \\
\hline$\Delta \mathrm{C}$-peptide $(\mathrm{ng} / \mathrm{ml})$ & $1.12 \pm 0.28$ & $2.09 \pm 0.97$ & $1.24 \pm 0.56$ & $<0.001$ \\
\hline Fasting insulin (IU) & $15.04 \pm 9.46^{\mathrm{a}}$ & $21.72 \pm 18.71^{\mathrm{ab}}$ & $22.54 \pm 33.94^{\mathrm{b}}$ & 0.049 \\
\hline Postprandial insulin (IU) & $30.33 \pm 20.01^{\mathrm{a}}$ & $51.46 \pm 43.96^{\mathrm{b}}$ & $34.5 \pm 39.04^{\mathrm{a}}$ & 0.001 \\
\hline$\Delta$ Insulin (IU) & $14.93 \pm 11.05$ & $28.16 \pm 20.44$ & $18.27 \pm 9.12$ & $<0.001$ \\
\hline Total cholesterol (mg/dl) & $213.52 \pm 52.25$ & $209.04 \pm 44.24$ & $212.21 \pm 44.5$ & 0.863 \\
\hline $\mathrm{HDL}(\mathrm{mg} / \mathrm{dl})$ & $39.71 \pm 8.4$ & $39.5 \pm 11.53$ & $42.17 \pm 17.69$ & 0.340 \\
\hline $\mathrm{LDL}$ (mg/dl) & $133.42 \pm 38.66$ & $127.52 \pm 33.37$ & $133.54 \pm 35.59$ & 0.593 \\
\hline Triglyceride (mg/dl) & $237.16 \pm 221.91$ & $227.78 \pm 185.07$ & $205.07 \pm 112.44$ & 0.448 \\
\hline Vitamin D (ng/ml) & $12.79 \pm 9.23$ & $11.86 \pm 7.63$ & $13.42 \pm 7.71$ & 0.569 \\
\hline Diabetes duration (years) & $12.99 \pm 6.54^{\mathrm{a}}$ & $10.87 \pm 6.75^{\mathrm{b}}$ & $15.12 \pm 9.46^{\mathrm{b}}$ & 0.024 \\
\hline
\end{tabular}

DSIT = Diverted sleeve gastrectomy with ileal transposition; SG = Sleeve gastrectomy; MGB = Mini-gastric bypass; $\mathrm{BMI}=$ Body mass index; $\mathrm{HbA}_{1 \mathrm{C}}=$ Hemoglobin $\mathrm{A}_{1 \mathrm{C}} ; \mathrm{HDL}=$ High-density lipoprotein; LDL = Low-density lipoprotein.

a-b-c: Different superscripts indicate statistically significant difference. Groups having the same superscripts are associated with similar results. 
TABLE 12.2: Type of medications used in all groups before surgery

\begin{tabular}{|l|l|l|l|}
\hline Medication & DSIT $(\mathbf{n}=\mathbf{1 0 9})$ & SG $(\mathbf{n}=49)$ & MGB (n=93) \\
\hline OAD only & $21(19.2 \%)$ & $28(57.1 \%)$ & $17(18.3 \%)$ \\
\hline Insulin only & $17(15.6 \%)$ & $4(8.2 \%)$ & $9(9.7 \%)$ \\
\hline OAD + insulin & $71(65.2 \%)$ & $17(34.7 \%)$ & $67(72 \%)$ \\
\hline
\end{tabular}

DSIT $=$ Diverted sleeve gastrectomy with ileal transposition; SG = Sleeve gastrectomy; MGB = Mini-gastric bypass.

$80.8 \%$ of patients in DSIT, $42.9 \%$ of patients in SG, and $81.7 \%$ of patients in MGB group were using insulin with/ without oral antidiabetics (OAD) ( $\mathrm{p}<0.001$ for SG vs. MGB and DSIT, $\mathrm{p}=0.426$ for DSIT vs. MGB). Note: 1 patient in the DSIT, and 4 patients in the MGB group were using OAD + insulin + GLP-1 analogue (Liraglutide).

\section{GLYCEMIC CONTROL AFTER SURGERY}

Group analysis showed that 13 of 109 patients (11.92\%) undergoing DSIT, 16 of 93 patients (17.2\%) undergoing MGB, and 11 of 49 patients (22.44\%) undergoing SG had normal plasma glucose levels on the morning of surgery. On postoperative day 30; 81 of 109 patients $(74.31 \%)$ treated by DSIT, 66 of 93 patients (70.96\%) treated by MGB, and 29 of 49 patients (59.18\%) treated by SG had normal glucose levels $(<126 \mathrm{mg} / \mathrm{dL})$ at least once, within 30 days after surgery. Graphical demonstration of the percentage of patients reaching normoglycemia, comparing the first and $30^{\text {th }}$ days is shown in Figure 12.2.

Figure 12.3 provides an overview of the daily (morning/evening) glucose measurements in the first 30 days after surgery. On the first day after surgery, 40 patients (15.93\%), and on the $30^{\text {th }}$ day, 176 patients $(70.11 \%)$, achieved normoglycemia defined as a blood glucose level of $\leq 126 \mathrm{mg} / \mathrm{dL}$. The mean plasma glucose level of all patients was $177.63 \pm 51.3 \mathrm{mg} / \mathrm{dL}$ on the morning of surgery, and $131.35 \pm 28.7 \mathrm{mg} / \mathrm{dL}$ on postoperative day 30 . When type of surgery was assessed, the SG group never achieved a mean plasma glucose level $<126 \mathrm{mg} / \mathrm{dL}$. Although 29 of 49 patients (59.18\%) in this group had blood glucose level $<126 \mathrm{mg} / \mathrm{dl}$, at least once during this period, the average glucose value remained above $126 \mathrm{mg} / \mathrm{dl}$.

The lowest mean fasting plasma glucose level in the SG group was $132.84 \pm 42.4 \mathrm{mg} / \mathrm{dL}$, occurring on the $30^{\text {th }}$ day. A mean fasting plasma glucose level of $<126 \mathrm{mg} / \mathrm{dL}$ was achieved on the evening of day 29 for DSIT $(124.36 \pm 20.21 \mathrm{mg} / \mathrm{dL})$, and on the evening of day 30 for MGB (123.61 $\pm 22.51 \mathrm{mg} / \mathrm{dL})$.

\section{MULTIVARIATE ANALYSIS}

Multivariate logistic regression analysis was used to determine the independent relationship between surgery type and changes in weight and plasma glucose levels. When performing a multivariate logistic regression analysis on glycemic control after DSIT (compared with the 


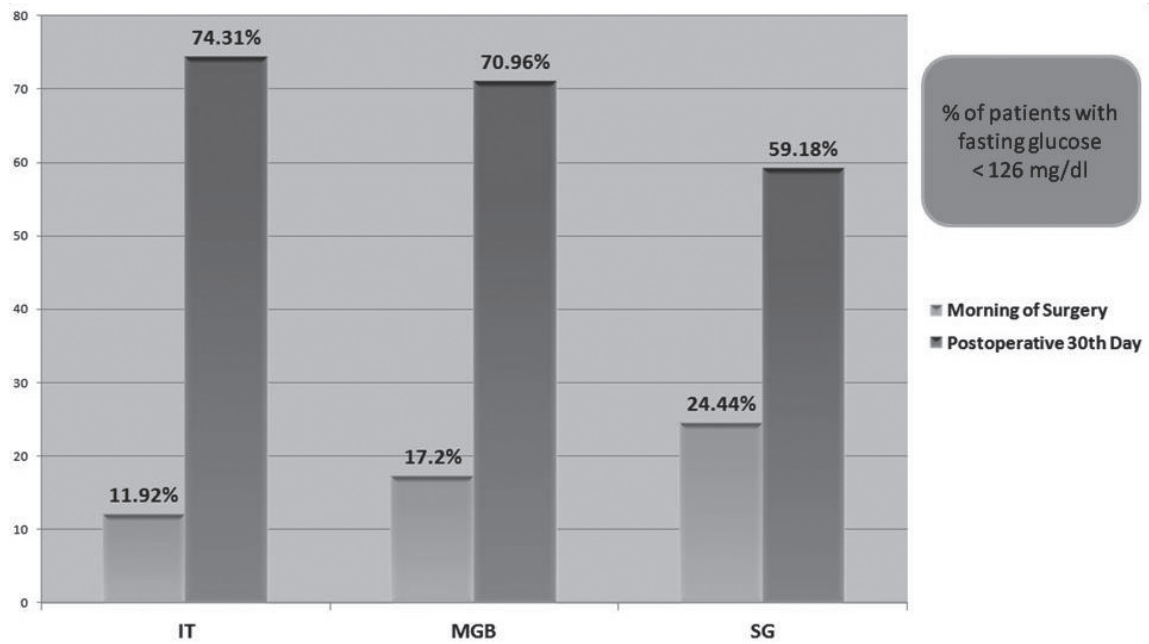

FIGURE 12.2. Percentage of patients who achieved normoglycemia.

(blood glucose level $\leq 126 \mathrm{mg} / \mathrm{dL}$ ) with different surgical modalities, both on the morning of surgery and on POD 30 Patients treated by DSIT had the highest rate of glycemic improvement, followed by MGB and SG. In the SG group, 29 of 49 patients (59.18\%) had blood glucose level $<126 \mathrm{mg} / \mathrm{dl}$, at least once during this period. However, the average glucose value for this group persisted above $126 \mathrm{mg} / \mathrm{dl}$.

$D S I T=$ Diverted sleeve gastrectomy with ileal transposition; $M G B=$ Mini-gastric bypass; $S G=$ Sleeve gastrectomy.

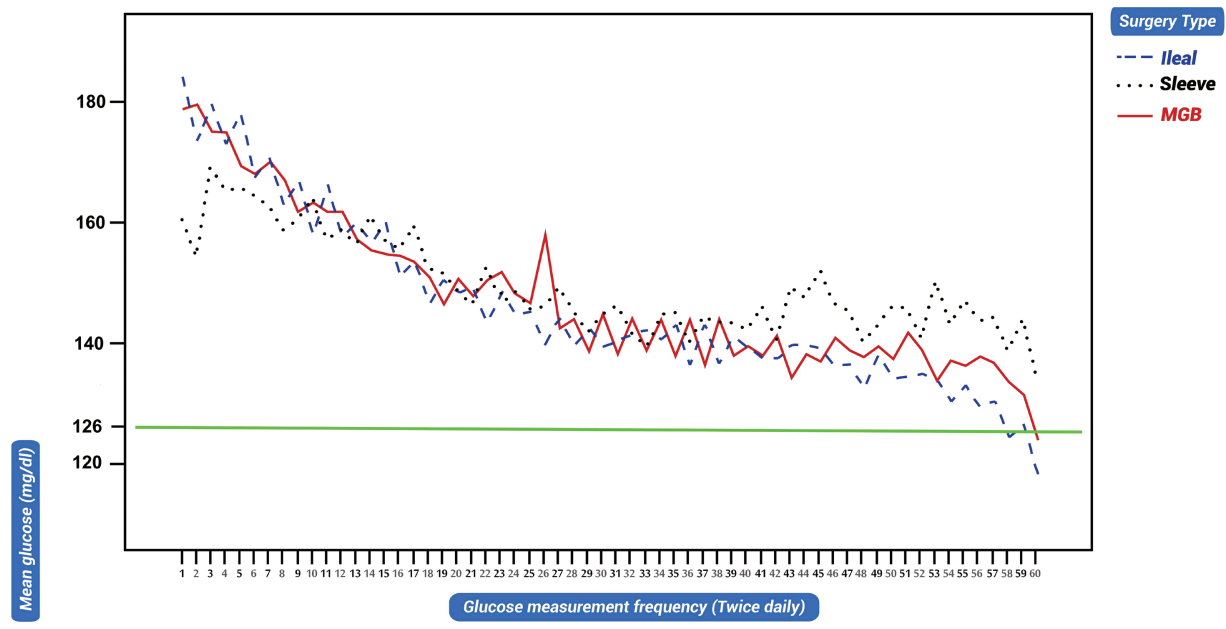

FIGURE 12.3. Daily follow-up mean glycemic level in all groups.

Horizontal green line indicates normoglycemia $(126 \mathrm{mg} / \mathrm{dl})$. Duplicate measures per day were obtained in all groups. Glucose levels were significantly lower in the SG compared to DSIT and MGB groups (SG, 161.82 \pm 44.2 $\mathrm{mg} / \mathrm{dL}$; DSIT, $183.97 \pm 47.39 \mathrm{mg} / \mathrm{dL}$; MGB, $178.52 \pm 57.58 \mathrm{mg} / \mathrm{dL}$ ) on postoperative Day 1 . Starting from day 27, DSIT and MGB groups experienced significantly lower glucose levels compared to the SG group. Within the first 30 days, the DSIT and MGB groups had mean glucose levels $<126 \mathrm{mg} / \mathrm{dL}$. Group effect: $\mathrm{p}=0.828$; Time effect: $\mathrm{p}<0.001$; Group x Time effect: $\mathrm{p}<0.001$. Repeated measures ANOVA. 
SG group as reference), preoperative BMI (OR $=0.855 ; 95 \%$ CI [0.753 - 0.957]; $\mathrm{p}=0.003)$ and postprandial C-peptide $(\mathrm{OR}=0.542 ; 95 \% \mathrm{CI}[-0.036-1.120] ; \mathrm{p}=0.038)$ were found to be independent predictors for glycemic control after surgery. When performing a multivariate logistic regression analysis on glycemic control after MGB (compared with the SG group as reference), no significant independent predictors were found.

\section{Patient Follow-up Chart}

\begin{tabular}{|c|c|c|c|c|c|c|c|c|c|}
\hline \multicolumn{7}{|c|}{ Name and Surname: } & \multicolumn{3}{|c|}{ Starting Date: / / 20} \\
\hline Day & $\begin{array}{l}\text { Fasting } \\
\text { Blood } \\
\text { Sugar } \\
\text { (Morning) }\end{array}$ & $\begin{array}{l}\text { Fasting } \\
\text { Blood } \\
\text { Sugar } \\
\text { (Evening) }\end{array}$ & Weight & $\begin{array}{l}\text { Blood } \\
\text { Pressure } \\
\text { (Morning) }\end{array}$ & $\begin{array}{l}\text { Daily Wa- } \\
\text { ter Intake }\end{array}$ & $\begin{array}{l}\text { Number of } \\
\text { Urination }\end{array}$ & $\begin{array}{c}\text { Breakfast } \\
+ \\
\text { Snack }\end{array}$ & $\begin{array}{c}\text { Lunch } \\
+ \\
\text { Snack }\end{array}$ & $\begin{array}{c}\text { Dinner } \\
+ \\
\text { Snack }\end{array}$ \\
\hline \multicolumn{10}{|l|}{1} \\
\hline \multicolumn{10}{|l|}{2} \\
\hline \multicolumn{10}{|l|}{3} \\
\hline \multicolumn{10}{|l|}{4} \\
\hline \multicolumn{10}{|l|}{5} \\
\hline \multicolumn{10}{|l|}{6} \\
\hline \multicolumn{10}{|l|}{7} \\
\hline \multicolumn{10}{|l|}{8} \\
\hline \multicolumn{10}{|l|}{9} \\
\hline \multicolumn{10}{|l|}{10} \\
\hline \multicolumn{10}{|l|}{11} \\
\hline \multicolumn{10}{|l|}{12} \\
\hline \multicolumn{10}{|l|}{13} \\
\hline \multicolumn{10}{|l|}{14} \\
\hline \multicolumn{10}{|l|}{15} \\
\hline \multicolumn{10}{|l|}{16} \\
\hline \multicolumn{10}{|l|}{17} \\
\hline \multicolumn{10}{|l|}{18} \\
\hline \multicolumn{10}{|l|}{19} \\
\hline \multicolumn{10}{|l|}{20} \\
\hline \multicolumn{10}{|l|}{21} \\
\hline \multicolumn{10}{|l|}{22} \\
\hline \multicolumn{10}{|l|}{23} \\
\hline \multicolumn{10}{|l|}{24} \\
\hline \multicolumn{10}{|l|}{25} \\
\hline \multicolumn{10}{|l|}{26} \\
\hline 27 & & & & & & & & & \\
\hline 28 & & & & & & & & & \\
\hline 29 & & & & & & & & & \\
\hline 30 & & & & & & & & & \\
\hline
\end{tabular}

FIGURE 12.4. Postoperative follow-up chart 


\subsection{DISCUSSION}

In the present study, we aimed to analyze how soon patients achieve glycemic control after different bariatric surgical options. Our results demonstrated that the SG group did not achieve a mean fasting plasma glucose level $<126 \mathrm{mg} / \mathrm{dL}$ within the first 30 postoperative days. A mean plasma glucose level below $126 \mathrm{mg} / \mathrm{dL}$ was achieved on postoperative day 29 for DSIT and on postoperative day 30 for MGB. Also, multivariate logistic regression analysis identified preoperative BMI and postprandial C-peptide in the DSIT group as independent predictors for postoperative glycemic control. To our knowledge, this is the first study to compare short-term (first 30 days) glycemic control in three different types of surgery (DSIT, MGB and SG).

Many studies have investigated the metabolic outcomes of surgical treatment, but most have not focused on the short-term glycemic changes in obese T2DM patients with relatively newer surgical procedures such as DSIT $(16,17)$ and MGB (18). A recent systematic review and meta-analysis investigated whether remission of T2DM depends on the surgical procedure or BMI. The authors had three main findings regarding anthropometric variables: $i$ ) bariatric surgery improves T2DM to the same extent in patients with a BMI of greater versus less than $35 \mathrm{~kg} / \mathrm{m}^{2}$, ii) improvement in $\mathrm{HbA}_{1 \mathrm{C}}$ was independent of baseline BMI, and iii) improvement in $\mathrm{HbA}_{1 \mathrm{C}}$ was inversely related to waist circumference (19). In the current study, preoperative BMI was found to be an independent predictor of glycemic control in patients after the DSIT. According to the same article, biliopancreatic diversion with a duodenal switch (BPDDS) had the strongest effect on diabetic remission (89\%), followed by Roux-en-Y gastric bypass (RYGB) (77\%); whereas, both SG and laparoscopic adjustable gastric banding (LAGB) had similar remission rates (60\% and 62\%, respectively) (19). The effects of BPDDS and RYGB on diabetic remission were also confirmed by a systematic review and meta-analysis conducted by Buchwald et al (6).

As reported by Fried et al in an integrative review of metabolic surgery for the treatment of T2DM in patients with $\mathrm{BMI}<35 \mathrm{~kg} / \mathrm{m}^{2}$, the resulting excessive weight loss was clinically meaningful, but not drastic (from a BMI of 29.4 to $24.2 \mathrm{~kg} / \mathrm{m}^{2}$ ). In spite of that, $85.3 \%$ of patients were free off T2DM medications and had a fasting plasma glucose level and $\mathrm{HbA}_{1 \mathrm{C}}$ approaching normal values. In a subgroup analysis, the authors found that BMI reduction and T2DM resolution were the greatest in malabsorptive procedures (20).

These results show that restrictive gastric procedures have lower diabetic remission rates than the procedures involving intestinal diversion in the long run. Also, procedures based on intestinal re-routing of the distal ileum provided more effective control than RYGB. In 
our study, both DSIT and MGB provided more effective glycemic control than SG despite yielding lower excessive weight loss than SG.

As an explanation of these results, the pathophysiology of T2DM may be revisited: Excessive weight and adipotoxicity issues are suggestive as only a single group of mechanisms leading to T2DM. In the novel paper, by De Fronzo, eight mechanisms have been pointed out to regulate the natural progression of T2DM, and among those, incretin resistance / insufficiency and alpha cells (glucagon activity) play major roles (21). In our study, we found that better glycemic control was achieved with intestinal re-routing procedures added to a mechanical restriction despite less excessive weight loss. Our findings may be reflecting the role of incretins and alpha cells.

In a population-based cohort study performed by Yska et al, T2DM remission rates after bariatric surgery were compared with those of a matched control group receiving medical therapy. Per 1,000 person-years, 94.5 T2DM remissions were found in the surgical group, compared with 4.9 in the control group. The surgical group of patients had an 18-fold increased chance of T2DM remission, compared with matched controls. The greatest effect was observed in RYGB patients (adjusted RR = 43.1), followed by SG (adjusted RR = 16.6) and gastric banding (adjusted $R R=6.9$ ) [22]. These findings were further supported in studies by Zanghelini et al and Gagner, et al (23-26).

The efficiency of surgical options based on mechanical restriction depends mainly on weight loss, which is expected to exert a positive effect on glycemic control. But, according to metaanalysis results, clinical success seems to be limited with surgical procedures like SG and LAGB, which are based on mechanical restriction. Long-term results obtained from diabetic patients who underwent SG showed that diabetic remission rates deteriorated in time, approaching $20 \%$ in five-year follow-up (27).

Major metabolic operations such as BPD, BPDDS, RYGB, duodenal-jejunal bypass (DJB) and IT are known for rapid postoperative glycemic control (28). Procedures based on intestinal re-routing of ileum, like BPD, have documented effects on reduction of hyperglycemia and improvement of insulin sensitivity as early as one month after surgery (29). RYGB, which is another type of metabolic surgery, promotes rapid improvement in T2DM irrespective of weight loss. Elevations in postprandial GLP-1 have been shown to be the triggering factors for increased insulin production within one week after surgery (30), and improved oral glucose tolerance one month after surgery (31).

A paucity of literature exists regarding T2DM remission after MGB. Musella et al. conducted a comparative study of MGB and SG in terms of T2DM remission. They found that T2DM was 
in remission in $85.4 \%$ of the MGB patients and $60.9 \%$ of SG patients in the first postoperative year $(\mathrm{p}<0.001)(32)$. High diabetic remission rates after MGB have been confirmed in a study by Kular et al (18).

The DSIT is a relatively new surgical procedure with promising outcomes. In a recent report, our group demonstrated that, at one year following surgery, DSIT had good metabolic outcomes in terms of T2DM remission, with a significant decrease in $\mathrm{HbA}_{1 \mathrm{C}}$, from $9.24 \%$ to $6.14 \%$ (17), and with a significant reduction in diabetic medication costs (14).

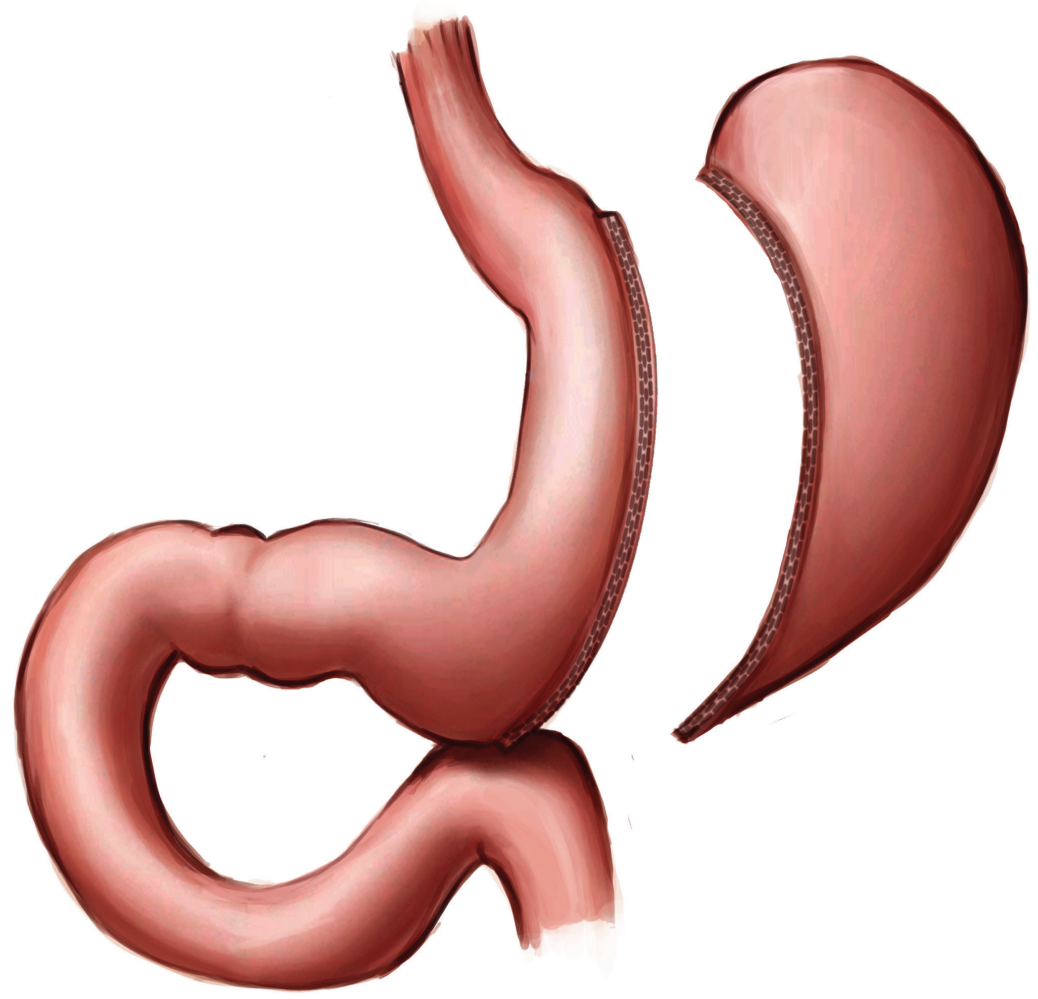

FIGURE 12.5. Types of included surgical procedures. A: Sleeve gastrectomy 


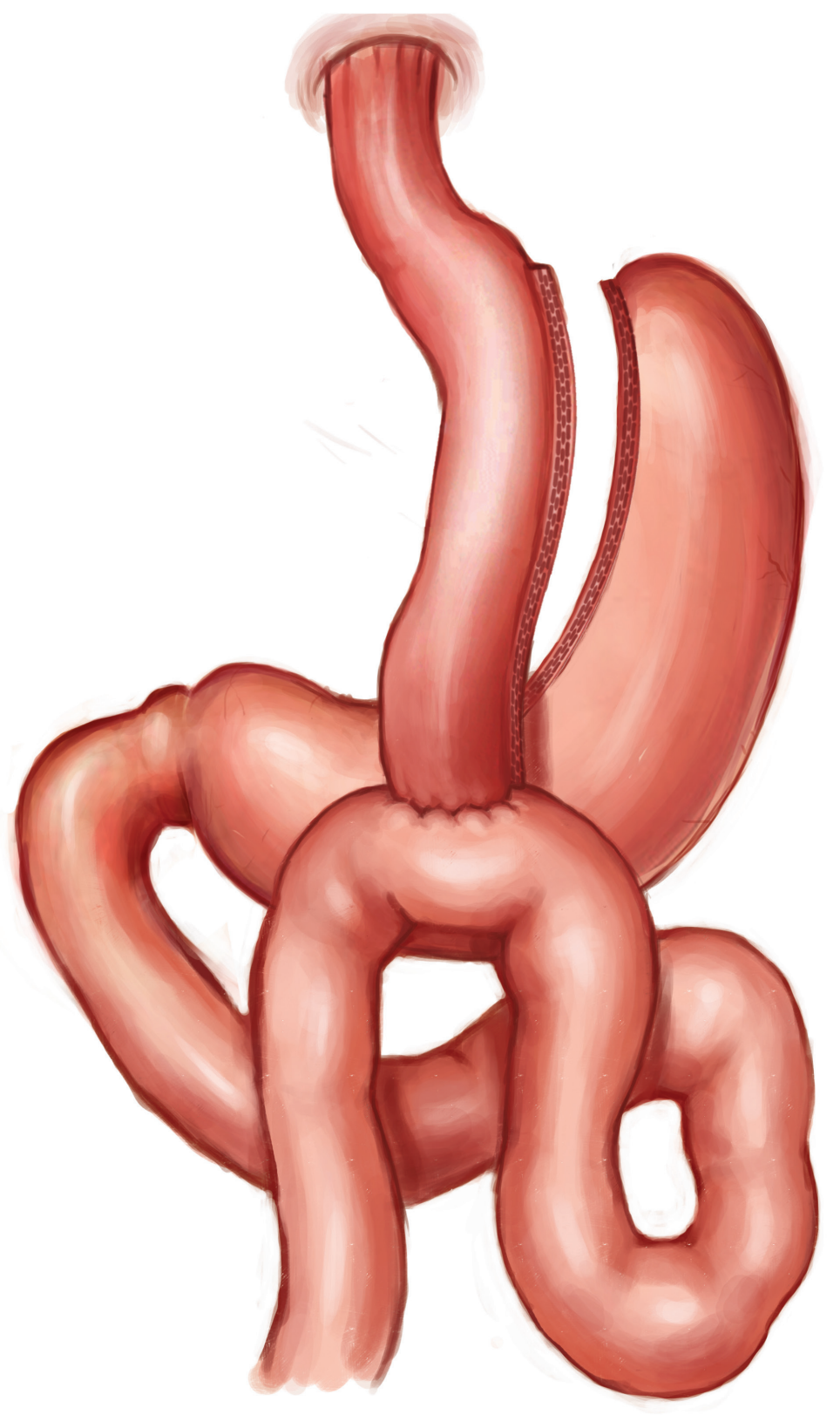

B: Mini Gastric Bypass (MGB) 


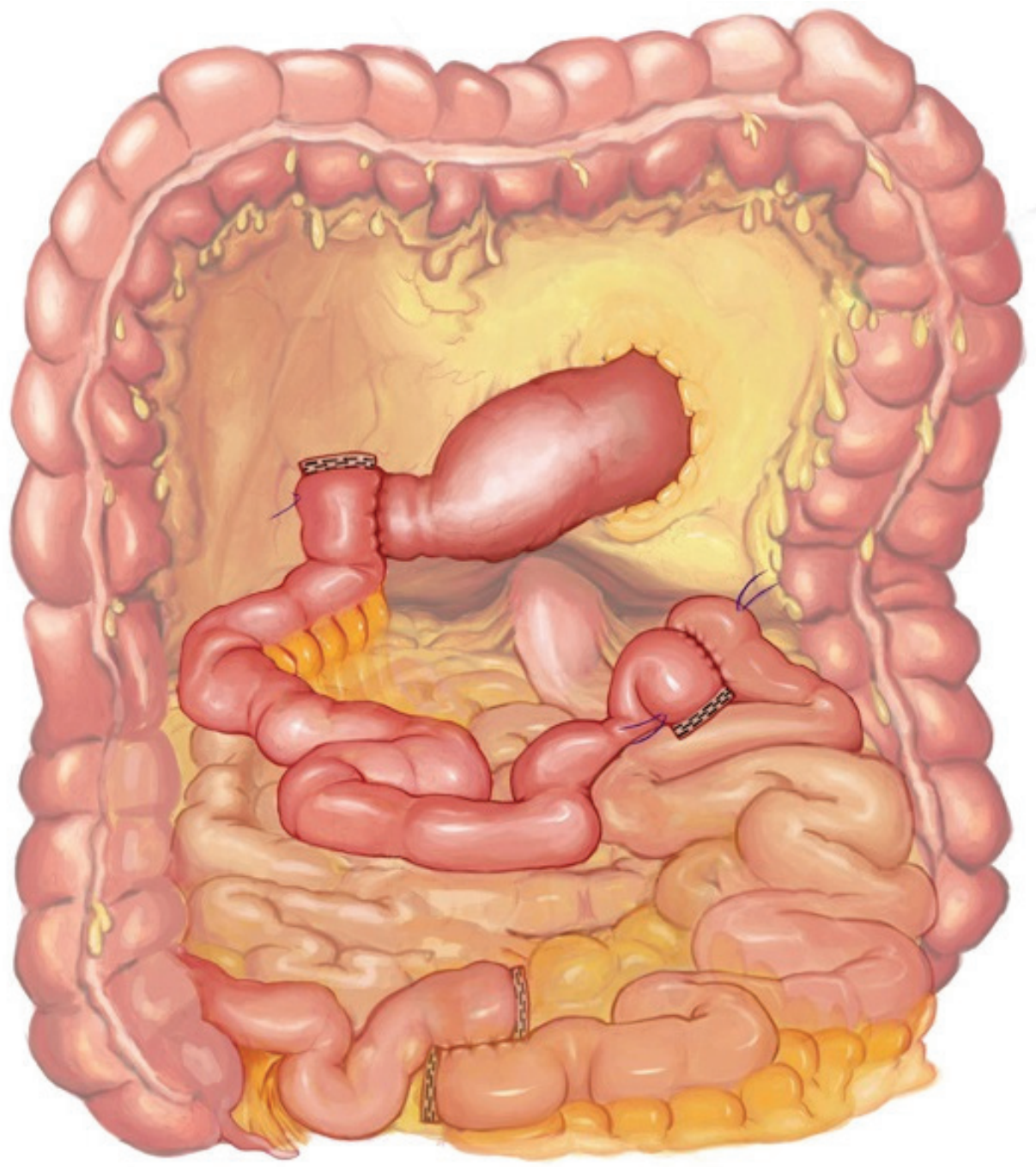

C: Diverted Sleeve Gastrectomy and ileum transposition (DSIT) 
Results of the current study demonstrated that, initially, the SG group had a markedly lower mean fasting glucose level on the morning of surgery. This outcome might result from lower $\mathrm{HbA}_{1 \mathrm{C}}$ and fructosamine levels, higher fasting, postprandial and $\Delta \mathrm{C}$-peptide, and insulin values, demonstrating a slightly lower disease severity, and better beta cell functions in this group. Furthermore, the rate of SG insulin users was markedly lower than that of the DSIT and MGB groups (42.9\% vs. $80.8 \%$ and $81.7 \%$ ), (Table 12.2). Despite these differences in baseline characteristics, the degree of weight loss was similar in all groups. While statistically not significant, the SG group had the highest level of weight loss. But, the change in plasma glucose levels was the lowest in the same group. The DSIT and MGB groups had almost identical weight loss patterns, but disease severity and beta cell functions were markedly better in the MGB group. Although the baseline characteristics reflected a higher disease severity and patients had a lower mean BMI in the DSIT group, the mean glucose level showed better improvement. Duodenal exclusion was performed in both DSIT and MGB groups. The distinctive efficiency in the DSIT group may be due to the early contact of food with ileal mucosa, and a more potent activation of the ileal hormones.

At the end of first month, 29 of 49 patients that underwent SG had blood glucose level $<126$ $\mathrm{mg} / \mathrm{dl}$ at least once in the postoperative period. However, the average glucose value for the group persisted above $126 \mathrm{mg} / \mathrm{dl}$. Therefore, it would be better to mention an improvement in glycemia rather than a frank glycemic control.

Apart from the type of surgical method, several scoring systems have been used to predict T2DM remission after bariatric surgery. The Dia-Rem score, for instance, includes factors such as insulin use, age, $\mathrm{HbA}_{1 \mathrm{C}}$ and type of antidiabetic medication (33). In another report, a diagnostic system that includes recent T2DM diagnosis, absence of insulin therapy regardless of weight loss, and definition of the remission were employed as the main predictors (34). The ABCD score (Age, BMI, C-peptide, duration) and weight loss have been reported to have a major influence on the outcomes (35). Although these scoring systems are used to determine long-term diabetic remission rates, when adapted to the current cohort of patients, there was no difference in age. The remaining determinants of diabetic remission favored SG, followed by MGB, and DSIT. Despite this fact, we observed better glycemic control in the DSIT group, followed by MGB, and SG.

This study has some limitations. First, the criteria for T2DM remission are quite different among different countries and also among other studies investigating T2DM remission after bariatric surgery. These definitions range from simple withdrawal of diabetes medication to fasting glucose levels $<100 \mathrm{mg} / \mathrm{dL}$ or $<126 \mathrm{mg} / \mathrm{dL}$ and/or final levels of $\mathrm{HbA}_{1 \mathrm{C}}$ ranging from $<6 \%$ to $<7 \%(6,15,19)$. Such different definitions can create bias in interpreting results. A second limitation was that blood glucose controls were performed with a glucometer (glucose [mg/ $\mathrm{dL}]=0.102+[19.295 \mathrm{X}$ capillary blood glucose $(\mathrm{mg} / \mathrm{dL}) / 18])$ in accord with International 
Federation of Clinical Chemistry and Laboratory Medicine (IFCC) recommendations for devices measuring glucose levels in capillary blood samples after calibrated to plasma glucose levels.

Even though the calibrations of the devices used by the patients were controlled prior to their discharge, patient- or device-related errors could occur. Also, patients checked their weight at home using their own scales; biases related to this variable may have occurred. To minimize this, patients were weighed before surgery and on the same day of their control visit with the same scales, while their weight on the discharge was written on their charts as the baseline. For some patients with poorly controlled DM, poor glycemic control can be attributed to suboptimal glucose monitoring and treatment (Reviewer \#3, comment \#2). Furthermore, differences between initial BMI and oral/insulin dependent mix values in different groups and lack of long term follow-up may bring about remarkable restrictions on our results.

The sleeve gastrectomy patients in the present study had the lowest $\mathrm{Hb} \mathrm{A} 1 \mathrm{c}$ levels, despite the highest average BMI values initially. This may indicate that their compliance might be better before surgery and in such a case, this may be another factor which may influence our results. Notwithstanding these potential limitations, we observed the highest improvement of glucose levels in the DSIT group. Initially, this group had the highest mean glucose levels, but by the end of the $30^{\text {th }}$ day, they presented the lowest levels of mean blood glucose. A similar outcome was also evident for the MGB group. Since the mean number of manipulations and the procedure time were higher in the DSIT group, this group might also have had a greater inflammatory reaction and exaggerated surgical stress hormones. Although we did not evaluate the levels of these hormones, it is possible that restoration of the incretin effect due to intestinal re-routing might have been delayed, causing their effects to manifest by the end of the fourth week.

\subsection{CONCLUSION}

In conclusion, in this study, we observed differences in glycemic control following three types of surgery within the first 30 postoperative days. Patients in the sleeve gastrectomy group did not achieve a mean plasma glucose level $<126 \mathrm{mg} / \mathrm{dL}$. Mean plasma glucose levels $<126 \mathrm{mg} /$ $\mathrm{dL}$ were achieved on the evening of day 29 following diverted sleeve gastrectomy with ileal transposition, and on the evening of day 30 after the mini-gastric bypass. Multivariate logistic regression analysis identified preoperative BMI and postprandial C-peptide as independent predictors of postoperative glycemic control in the diverted sleeve gastrectomy with ileal transposition group. Intestinal rearrangement may provide an important component of diabetes control following bariatric surgery. Weight loss was not the only or perhaps primary contributor to early achievement of glycemic control. More research on this topic is needed. 


\subsection{REFERENCES}

1 Ng M, Fleming T, Robinson M, Thomson B, Graetz N, Margono C, et al. Global, regional, and national prevalence of overweight and obesity in children and adults during 1980-2013: a systematic analysis for the Global Burden of Disease Study 2013. Lancet. 2014;384(9945):766-81. Association AD. The dangerous toll of diabetes http://www.diabetes.org/diabetes-statistics/ dangerous-toll.jsp

3 Vaag AA. Glycemic control and prevention of microvascular and macrovascular disease in the Steno 2 study. Endocr Pract. 2006 Jan-Feb;12 Suppl 1:89-92.

4 Bermudez DM, Pories WJ. New technologies for treating obesity. Minerva endocrinologica. 2013; 38(2): 165-72.

5 Schauer PR, Kashyap SR, Wolski K, Brethauer SA, Kirwan JP, Pothier CE, et al. Bariatric surgery versus intensive medical therapy in obese patients with diabetes. The New England journal of medicine. 2012;366(17):1567-76.

6 Buchwald H, Estok R, Fahrbach K, Banel D, Jensen MD, Pories WJ, et al. Weight and type 2 diabetes after bariatric surgery: systematic review and meta-analysis. The American journal of medicine. 2009;122(3):248-56 e5.

7 Vetter ML, Cardillo S, Rickels MR, Iqbal N. Narrative review: Effect of bariatric surgery on type 2 diabetes mellitus. Ann Int Med. 2009;150(2):94-103.

8 Romeo S, Maglio C, Burza MA, Pirazzi C, Sjoholm K, Jacobson P, et al. Cardiovascular events after bariatric surgery in obese subjects with type 2 diabetes. Diabetes Care. 2012;35(12):2613-7.

9 Adams TD, Gress RE, Smith SC, Halverson RC, Simper SC, Rosamond WD, et al. Long-term mortality after gastric bypass surgery. N Engl J Med. 2007;357(8):753-61.

10 Sjostrom L, Narbro K, Sjostrom CD, Karason K, Larsson B, Wedel H, et al. Effects of bariatric surgery on mortality in Swedish obese subjects. New Engl J Med. 2007;357(8):741-52.

11 Fenske WK, Pournaras DJ, Aasheim ET, Miras AD, Scopinaro N, Scholtz S, et al. Can a protocol for glycaemic control improve type 2 diabetes outcomes after gastric bypass? Obes Surg. 2012; 22(1):90-6.

12 Briatore L, Salani B, Andraghetti G, Danovaro C, Sferrazzo E, Scopinaro N, et al. Restoration of acute insulin response in T2DM subjects 1 month after biliopancreatic diversion. Obesity (Silver Spring, Md). 2008;16(1):77-81.

13 Briatore L, Salani B, Andraghetti G, Maggi D, Adami GF, Scopinaro N, et al. Beta-cell function improvement after biliopancreatic diversion in subjects with type 2 diabetes and morbid obesity. Obesity (Silver Spring, Md). 2010;18(5):932-6.

14 Celik A, Asci M, Celik BO, Ugale S. The impact of laparoscopic diverted sleeve gastrectomy with ileal transposition (DSIT) on short term diabetic medication costs. SpringerPlus. 2015; 14(4): 417.

15 Expert Committee on the Diagnosis and Classification of Diabetes Mellitus. Report of the expert committee on the diagnosis and classification of diabetes mellitus. Diabetes Care. 2003;26 Suppl $1:$ S5-20. 
16 Celik A, Ugale S, Ofluoglu H, Asci M, Celik BO, Vural E, et al. Technical feasibility and safety profile of laparoscopic diverted sleeve gastrectomy with ileal transposition (DSIT). Obes Surg. 2015;25(7):1184-90.

17 Celik A, Ugale S, Ofluoglu H, Vural E, Cagiltay E, Cat H, et al. Metabolic outcomes of laparoscopic diverted sleeve gastrectomy with ileal transposition (DSIT) in obese type 2 diabetic patients. Obes Surg. 2015;25(11):2018-22.

18 Kular KS, Manchanda N, Cheema GK. Seven years of mini-gastric bypass in type 2 diabetes patients with a body mass index $<35 \mathrm{~kg} / \mathrm{m}^{2}$. Obes Surg. 2015 Oct 16. [Epub ahead of print]

19 Panunzi S, De Gaetano A, Carnicelli A, Mingrone G. Predictors of remission of diabetes mellitus in severely obese individuals undergoing bariatric surgery: do BMI or procedure choice matter? A meta-analysis. Annals of surgery. 2015;261(3):459-67.

20 Fried M, Ribaric G, Buchwald JN, Svacina S, Dolezalova K, Scopinaro N. Metabolic surgery for the treatment of type 2 diabetes in patients with BMI $<35 \mathrm{~kg} / \mathrm{m}^{2}$ : an integrative review of early studies. Obes Surg. 2010;20(6):776-90.

21 Defronzo RA. Banting Lecture. From the triumvirate to the ominous octet: a new paradigm for the treatment of type 2 diabetes mellitus. Diabetes. 2009;58(4):773-95.

22 Yska JP, van Roon EN, DeBoer A, Leufkens G, Wilffert B, Heide L, et al. Remission of type 2 diabetes mellitus in patients after different types of bariatric surgery: A population-based cohort study in the United Kingdom. JAMA Surg. 2015 Dec 1;150(12):1126-33.

23 Zanghelini F, Buehler AM, Pereira T. Efficacy and safety bariatric surgery vs. clinical treatment in control of levels glycemic and remission diabetes mellitus type II in patients obese. Value Health. 2015 Nov;18(7):A599.

24 Gagner M. Bariatric surgery vs lifestyle intervention for type 2 diabetes mellitus. JAMA Surg. 2015 Oct;150(10):940.

25 Gagner M. Safety and efficacy of a side-to-side duodeno-ileal anastomosis for weight loss and type-2 diabetes: duodenal bipartition, a novel metabolic surgery procedure. Ann Surg Innov Res. 2015 Oct 14;9:6.

26 Gagner M. Laparoscopic sleeve gastrectomy with ileal interposition (SGIT): a modified duodenal switch for resolution of type 2 diabetes mellitus in lesser obese patients (BMI < 35). World J Surg. 2011 Jan;35(1):109-10.

27 Golomb I, Ben David M, Glass A, Kolitz T, Keidar A. Long-term metabolic effects of laparoscopic sleeve gastrectomy. JAMA Surg. 2015 Nov;150(11):1051-7.

28 Papamargaritis D, Miras AD, le Roux CW. Influence of diabetes surgery on gut hormones and incretins. Nutr Hosp. 2013 Mar;28 Suppl 2:95-103.

29 Chiellini C, Rubino F, Castagneto M, Nanni G, Mingrone G. The effect of bilio-pancreatic diversion on type 2 diabetes in patients with BMI $<35 \mathrm{~kg} / \mathrm{m}^{2}$. Diabetologia. 2009;52(6):1027-30.

30 Pournaras DJ, Osborne A, Hawkins C, Vincent RP, Mahon D, Ewings P, et al. Remission of type 2 diabetes after gastric bypass and banding: mechanisms and 2 year outcomes. Ann Surg. 2010;252(6):966-71. 
31 LaFerrere B, Teixeira J, McGinty J, Tran H, Egger JR, Colarusso A, et al. Effect of weight loss by gastric bypass surgery versus hypocaloric diet on glucose and incretin levels in patients with type 2 diabetes. J Clin Endocrinol Metab 2008;July 93(7): 2479-85.

32 Musella M, Apers J, Rheinwalt K, Ribeiro R, Manno E, Greco F, et al. Efficacy of bariatric surgery in type 2 diabetes mellitus remission: The role of mini-gastric bypass/one anastomosis gastric bypass and sleeve gastrectomy at 1 year of follow-up. A European survey. Obes Surg. 2015;Sep 4. [Epub ahead of print]

33 Still CD, Wood GC, Benotti P, Petrick AT, Gabrielsen J, Strodel WE, et al. Preoperative prediction of type 2 diabetes remission after Roux-en-Y gastric bypass surgery: a retrospective cohort study. Lancet Diabetes Endocrinol. 2014 Jan;2(1):38-45.

34 Blackstone R, Bunt JC, Cortés MC, Sugerman HJ. Type 2 diabetes after gastric bypass: remission in five models using $\mathrm{HbA}_{1 \mathrm{C}}$, fasting blood glucose, and medication status. Surg Obes Relat Dis. 2012;8(5):548-55.

35 Lee MH, Lee WJ, Chong K, Chen JC, Ser KH, Lee YC, Chen SC. Predictors of long-term diabetes remission after metabolic surgery. J Gastrointest Surg. 2015;19(6):1015-21. 


13. SUMMARY, DISCUSSION AND FUTURE PERSPECTIVES 

Walter Pories made game-changing statements in 1992 (1) and 1995 (2). He suggested that bariatric surgery has more beneficial effects than solely weight loss. The statement that type 2 diabetes mellitus (T2DM) might be a surgical disease was a visionary one during that time. (1) By now the benefits of bariatric surgery for cardiovascular, pulmonary and metabolic diseases can be added. (3-7) The purpose of this thesis was to provide more insight in perioperative cardiopulmonary and metabolic physiology in bariatric surgery.

\section{PART I: CARDIAC AND PULMONARY PHYSIOLOGY}

An overview of the current literature about cardiac structure and function changes before and after bariatric surgery is provided in Chapter 2. In summary, irrespective of the type of bariatric surgical procedure, it seems that bariatric surgery is beneficial for myocardial structure, systolic and diastolic function. (8) Pre-existing hypertension and left ventricular hypertrophy seem to be a prerequisite for reverse remodelling after bariatric surgery. However the question remains whether cardiac pathology can be considered an indication for bariatric surgery. According to the guidelines of the International Federation of Surgery for Obesity and Metabolic disorders (9) cardiac pathology solely is not an indication for bariatric surgery, but several case series have demonstrated the positive effect of bariatric surgery on left ventricular ejection fraction (LVEF) and the New York Heart Association functional class (of heart failure). (10-14) Ristow et al. (15) reported on two patients who no longer required heart transplantation after successful weight reduction and improvement of LVEF.

The responsible mechanism(s) of cardiovascular changes after bariatric surgery are not fully clarified. (8) There might be a combination of weight-dependent (e.g. weight loss, circulating volume) and also weight-independent mechanisms (inotropic hormones, like GLP-1) that induce cardiac remodelling. This includes that there is a possibility of direct gut hormonal inotropic action on the myocardium through an enterocardiac axis. $(8,16)$ Manipulation of enteric gut hormones has been shown to have beneficial effects on cardiovascular function through the enterocardiac axis. $(16,17)$ The classical hemodynamic weight-dependent effect of bariatric surgery is no longer thought to be the only one responsible for reverse remodelling seen after bariatric surgery, as the beneficial effects can be independent from changes in blood pressure. $(18,19)$ Although these mechanisms are only understood partially, changes in blood pressure (e.g. remission of hypertension) can be beneficial for the burden of disease.

Hypertension, one of criteria for the metabolic syndrome, is a major contributor for the development of congestive heart failure. (20) In assessing the blood pressure (BP) of obese subjects several challenges exist. Nowadays, we have the standard auscultatory sphygmomanometry, widely used in clinical practice, based on the principles of Riva-Rocci/ 
Korotkoff (RRK). (21) However, RRK BP measurements can lead an overestimation of the BP of obese patients due to the cuff size and the circumference of the upper arm. $(22,23)$ This could induce misdiagnosing and over-treating hypertension in this population. (22-24) The increasing prevalence of obesity makes validation of finger blood pressure measurements for this group clinical valuable, because blood pressure cuff readings as well as intra-arterial catheter placement in (morbid) obese individuals can be challenging or even impossible. (25) Fingers remain relatively thin in obesity, which may imply that finger blood pressure measurements using the volume clamp methodology may be feasible. The difficulty to assess, both invasive and non-invasive blood pressure in morbid obese individuals makes an alternative technique very valuable.

Therefore in chapter 3 , the Nexfin ${ }^{\circledR}$ non-invasive continuous hemodynamic monitoring device was validated against blood pressure measurement according to Riva-Rocci/Korotkoff in obese patients scheduled for bariatric surgery, using the original and the revised protocol of the European Society of Hypertension. This protocol consisted of two phases. The first phase determines how accurate the device will be for individual measurements and second phase 2.2 determines the accuracy for individual measurements. Determining the number of differences (between the Nexfin ${ }^{\circledR}$ device and RRK BP measurements) within 5, 10 and 15 $\mathrm{mmHg}$, and then determining the accuracy will do this. (26)

It was found that the Nexfin ${ }^{\circledast}$ device passed phase 1 and 2.1 of the original validation protocol developed by the European Society of Hypertension. (27) When comparing the obtained data with the revised protocol (26), the Nexfin ${ }^{\otimes}$ device was not able to pass part 1 and 2 of the validation (the former phase 2.1 and 2.2). Notwithstanding these results, the Nexfin ${ }^{\circledR}$ device could be suitable as a non-invasive monitoring device. This because the systolic BP recorded at the wrist may better reflect 'true' systolic BP prevailing in the artery than the systolic BP measured at the arm. (28) This possibility is supported in the study by de Senarclens et al. (28) by the observation that the ratio of width (the decimal log arm of circumference calculated in obese patients) was higher than the optimal value originally validated by Marks et al. (24) This basically means that in our study the arm systolic blood pressure is an overestimation of the 'true' blood pressure. Secondly, the reference method used could be another reason for not passing the validation protocol, because of the possible overestimation of the BP of our study population (as RRK BP measurements were used as a reference). This also explains why there is less differences between the Nexfin ${ }^{\circledR}$ measurements and other reference methods (such as radial artery blood pressure, used in a recent study by De Wilde et al. (29)) Thirdly, both protocols $(26,27)$ do not make any recommendations for special groups like patients with obesity. 
Despite not passing validation completely, the Nexfin ${ }^{\circledR}$ device is a valuable tool for assessing hemodynamic changes. Therefore in the follow-up study short-term hemodynamic changes before and three months after bariatric surgery were assessed using this device (chapter 4). It was found that irrespective of the type of bariatric surgery, that heart rate; mean arterial pressure (MAP), systolic and diastolic BP significantly decreased after bariatric surgery. And stroke volume significantly increased. These results are in line with the current literature, which shows that bariatric surgery has positive effects on cardiovascular hemodynamics. (30)

In addition to cardiovascular effects of bariatric surgery the effects on the pulmonary function were investigated. Chapter 5 gave an overview of the current literature regarding pulmonary physiology in patients with obesity and its considerations for perioperative care in bariatric surgery. Physiologically, obesity is associated with an altered lung function, characterised by a reduction of lung volumes, mostly a restrictive pattern. The pathogenesis is multifactorial, however an increased truncal fat load is one of the possible mechanisms. (31)

Because of affected respiratory physiological parameters such as compliance, neuromuscular strength, work of breathing, lung volumes and spirometric measurements $(32,33)$, obese subjects are prone to develop pulmonary complications after bariatric surgery. Several strategies are present to improve perioperative care, such as fast track (ERAS) programs. However, the most optimal perioperative care needs to be evaluated on a case-to-case basis. Regarding the link between the impaired lung function, postoperative complications and the necessity of pulmonary function screening prior to bariatric surgery, conflicting results exist and future studies need to determine whether preoperative pulmonary function screening is useful in bariatric practice.

One of the most important pulmonary function parameters is the maximum inspiratory pressure (MIP). It is known that a decreased MIP has a significant effect on postoperative pulmonary complications after cardiac surgery. (34-37) To investigate if this also applies after bariatric surgery, a study was performed to assess the MIP changes and a possible relationship between the MIP and postoperative pulmonary complications following bariatric surgery. In chapter 6 , the maximum inspiratory pressure (to assess inspiratory muscle strength) before and after bariatric surgery was determined. The maximum inspiratory pressure significantly decreased three months after bariatric surgery. After six and 9 months a gradual recovery/ increase was seen. Morbidly obese patients have increased metabolic demands due to a deposition of fat in the chest wall, which results in an increased mass to move and therefore a higher work of breathing (WOB). This elevated WOB results in reduced chest wall compliance. Also there is an elevation of the diaphragm, which (upon contracting) acts under pressure of a distended abdomen. (38-40) This pathophysiological mechanism is known as the 'overload' hypothesis and it is thought that the 'overload' triggers a variety of mechanisms 
in the activity of the respiratory muscles and causes a long-term training effect, which can increase muscle strength. $(38,39,41)$ In the postoperative period, weight reduction may promote an improvement in respiratory mechanics and compliance, improving the efficiency of the respiratory muscles. (42) The decrease in muscle strength (e.g. MIP) after three months is due to 1) a loss of fat free mass after surgery, roughly 10\% (43) 2) a more efficient cellular working mechanism shortly after bariatric surgery. (43) After six months a new cellular setpoint (in energy metabolism) will be achieved and therefore will increase inspiratory muscle strength.

Changes in maximal inspiratory muscle strength are one of the important pulmonary function alterations after bariatric surgery. Training goals to optimize the MIP before surgery are important as seen in cardiac surgery (34-37). In the current literature there are several predictive equations available, although not tailored for the obese/bariatric population and therefore difficult to predict changes in inspiratory muscle strength.

Chapter 7 gives a critical appraisal of the utility and adequacy of the currently available predictive equations. The equations of Harik-Khan, Neder, Costa, Wilson and Enright were studied.

In the preoperative period, the predicted maximum inspiratory pressure (MIP) according to the Harik-Khan, Neder, Costa and Wilson equation were significantly different compared to the Actual MIP ( $\mathrm{p}<0.05)$. The predicted MIP according to the Enright equation was not significantly different $(\mathrm{p}>0.05)$. Bland Altman analysis showed that the Enright equation was best suitable for predicting the MIP. In bariatric surgery, these equation are not often used, but as a preparation for cardiac surgery, these MIP equations can be suitable to determine adequate training goals. Inspiratory muscle training is effective in reducing postoperative pulmonary complications in patients scheduled for elective coronary artery bypass grafting. (34) In general, obesity is an increasing problem. (44) To determine the adequate training goal, there is increasing need for a mathematical equation which can adequately predict the respiratory muscle strength and therefore to set achievable training goals. According to this study, the equation of Enright et al. is the most adequate to predict MIP changes and therefore should be used in clinical practice.

Future research must get more insight in the respiratory function of the morbidly obese and the influence of bariatric surgery on respiratory muscle function several function parameters have to be studied. Spirometry is the first step for testing pulmonary function. A comparative analysis between spirometric variables and maximal inspiratory/expiratory pressures can provide more insight in the pulmonary physiology of the morbidly obese. 
To integrate energy metabolism, cardiac and pulmonary physiology, an overview of the current evidence regarding perioperative exercise in bariatric surgery was given in chapter 8 . Considering the great value of exercise, very limited number of prospective studies addressed perioperative exercise in bariatric surgery. To simplify our findings, an exercise program with a median of 12 weeks, an intensity of $65 \%$ peak heart rate $/ \mathrm{VO}_{2} \max$, with partial supervision, seems to be to most effective. However the literature regarding different perioperative exercise regimens is very sparse. Considering the earlier mentioned 'overload' hypothesis on pulmonary muscles, we can state that a same sort of 'overload' exists on skeletal muscles. This indicates a training effect of being obese on skeletal muscles and therefore high intensive interval training (HIIT) or resistance training with heavy weights should be beneficial to achieve adequate results. Regarding the timing of exercise regimens, controversies exist. (34, 45-48) Preoperative exercise seems more suitable because the effects of the training do not interact with the effects of bariatric surgery.

\section{PART II: METABOLLC PHYSIOLOGY}

The second part of this thesis focused on metabolic physiology, in particular nutritional deficiencies and type 2 diabetes (T2DM). An algorithm is suggested in Chapter 9 to improve and optimize the screening for nutrient deficiencies in patients admitted for bariatric surgery. In the development process of this algorithm the data of 561 Roux en-Y Gastric Bypass (RYGB) and 831 Sleeve Gastrectomy (SG) patients were included. These patients had to had at least pre- and postoperative values of vitamin A, B1, B6, B12, D, folate, ferritin, zinc or magnesium (with a mean follow-up ranging from 3 to 84 months postoperative. The algorithm reduces vitamin D, B12, B6, B1 and ferritin examinations by 15\%, 11\%, 28\%, 28\% and $38 \%$, respectively, without missing relevant deficiencies. The corresponding potential cost savings was $14 \%$. This algorithm shows us that we do not have to determine every vitamin and mineral in a preoperative laboratory screening to catch relevant deficiencies. It can be used until at least 84 months postoperative for RYGB and SG patients. Whether this algorithm is suitable for other bariatric procedures and for a follow-up of $>84$ months is subject for further research.

Chapters 10 and 11 focussed on the specific supplementation strategies for vitamin B12 deficiencies. In chapter 10, the variations in supplementation regimes to treat perioperative vitamin B12 deficiencies were systematically reviewed and analysed. Vitamin B12 supplementation has an effect on the intracellular vitamin B12 content and in the correct dosage it can prevent a vitamin B12 deficiency. There is no consensus about the correct dosage of vitamin B12 after bariatric surgery worldwide, as shown in the results of this study. (49-51) 
In chapter 11 is a study described wherein the effectiveness of three different intramuscular vitamin B12 supplementation regimes was assessed in a retrospective matched cohort study. One group received 6 intramuscular vitamin B12 injections with a loading dose; one group received 3 intramuscular vitamin B12 injections without loading dose and the third group received no vitamin $\mathrm{B} 12$ injections. Of these supplementation regimes, the 6 injections regime recovered all vitamin B12 deficiencies biochemically.

Following these two studies no optimal protocol of vitamin B12 supplementation can be suggested. Currently a lot of research is done to show with different supplementation regimens that vitamin and mineral deficiencies can be biochemically corrected after bariatric surgery, but somehow the clinical relevancy of 'correcting' these deficiencies is lost in the cause. In terms of reducing potential health risks, there is lack of evidence that a supplementation of vitamin B12 is beneficial. Therefore difficulties arise in defining hard endpoints in these supplementation trials. Secondly, especially for vitamin B12, we do not know whether a low or high dose supplementation has beneficial long terms effects. Current lacks in evidence comprise basically the link between deficiency and clinical relevancy, and the long-term effects of different supplementation regimens.

In terms of metabolic diseases studied, apart from vitamin and mineral physiology, there is great interest in the remission of T2DM after surgery. Especially the comparison between the well-established surgical procedures and the newer ones is an important subject nowadays. Therefore, in the final chapter of part II (chapter 12) assessed the time to glycemic control after several bariatric surgical procedures. In this study we aimed to determine how soon after bariatric/metabolic surgery patients achieve glycemic control (defined as a mean plasma glucose level $<126 \mathrm{mg} / \mathrm{dl}$ ) in different types of surgery. Our results demonstrated that in the first 30 days after surgery, the SG group never achieved a mean plasma glucose level $<126 \mathrm{mg} / \mathrm{dl}$. Mean plasma glucose level reaching below $126 \mathrm{mg} / \mathrm{dl}$ was achieved on $29^{\text {th }}$ day evening for Diverted Sleeve Gastrectomy and Ileal Transposition (DSIT), and $30^{\text {th }}$ day evening for Mini Gastric Bypass (MGB) patients.

Many studies have investigated the metabolic outcomes of surgical treatment, but most of them do not focus of the short-term glycemic changes in type 2 diabetes mellitus obese patients in relatively new surgical procedure like the DSIT (52-54) and MGB (55). Two problems arise interpreting results like type 2 diabetes remission; is the mechanism weight dependent or weight independent, and secondly how do you define remission of type 2 diabetes mellitus? A recent systematic review and meta-analysis by Panunzi et al. (56) investigated whether remission of T2DM is surgical procedure dependent or BMI dependent. They had three main findings regarding anthropometric variables: 1) bariatric surgery improves T2DM to the same extent in patients with a BMI of more or less than $35 \mathrm{~kg} / \mathrm{m}^{2} ; 2$ ) improvement 
of $\mathrm{Hb} 1 \mathrm{Ac}$ is independent of baseline $\mathrm{BMI} ; 3)$ improvement of $\mathrm{Hb} 1 \mathrm{Ac}$ is inversely related to waist circumference. (56) It seems that regarding the type of surgical procedure, there is a superiority of malabsorptive procedures in terms of type 2 diabetes mellitus remission. This was also shown by the meta-analysis of Panunzi et al. (56). They found that the biliopancreatic diversion with duodenal switch (BPD) has the strongest effect (89\% remission of T2DM), followed by the Roux en Y Gastric Bypass (RYGBP, 77\%), whereas both the SG and laparoscopic adjustable gastric banding (LAGB) had similar remission rates $(60 \%$ and $62 \%$ respectively). These results were confirmed by the systematic review and meta-analysis conducted by Buchwald and colleagues. (3)

\section{FUTURE PERSPECTIVES}

Many of the worlds leading bariatric surgeons stated (57) that we are ready for a change in the current guidelines regarding bariatric surgery. Secondly with the increased understanding of the remission of type 2 diabetes, we might even consider a change of name to metabolic surgery. A recent report by David Cummings and Ricardo Cohen support these earlier mentioned statements. (57)

According to the guidelines of the International Federation of Surgery for Obesity and Metabolic disorders (IFSO) (9) surgery is warranted to patients with a BMI greater than 40 $\mathrm{kg} / \mathrm{m}^{2}$, or greater than $35 \mathrm{~kg} / \mathrm{m} 2$ with serious comorbidities such as type 2 diabetes mellitus. Although these recommendations were clinically valuable, nowadays these guidelines have possible significant limitations and new scientific insights came to light. One of the limitations is the fact that only open operations were considered, whereas most procedures are now undertaken laparoscopically, which is safer with a ten-times lower operative mortality. $(58,59)$ A second limitation is that there were only moderate recommendations for diabetes, but nowadays increasing knowledge exists about the mechanisms responsible for improvement of type 2 diabetes. Thirdly no recommendations were made about the effects of bariatric surgery on cardiac and pulmonary diseases. This goes way beyond just reducing food intake and bodyweight. $(57,60)$ Therefore future research must focus on elucidating the mechanisms responsible for the remission of metabolic diseases and to clarify the gaps in current hypotheses.

We have to gain more insight in the effects of bariatric and metabolic surgery on the remission of cardiac and pulmonary diseases. Secondly bariatric and metabolic surgery can be of aid in preventing chronic pulmonary and cardiac diseases. Especially, chapters $2-8$ of this thesis give a basic insight in the effects of obesity and weight loss (due to bariatric surgery) on cardiac and pulmonary physiology. Furthermore, one of the future challenges lies in defining which imaging modality to assess the cardiac structure and functional parameters 
in patients with obesity. Currently there is no study available to assess the differences between echocardiography and cardiac magnetic resonance imaging (MRI). A recent study by Patel et al. (61) showed that the diagnostic performance of echocardiography might be inferior to cardiac MRI. In this study the diagnostic performance was tested in finding (para) cardiac masses, but the same diagnostic inconsistencies might occur in determining functional cardiac parameters in patients with obesity. Therefore the differences between cardiac MRI and echocardiography need to be assessed. Secondly as proposed in the chapter 2 of this thesis, the presence of left ventricular hypertrophy $(\mathrm{LVH})$ and/or hypertension are a prerequisite for cardiac remodelling. It seems obvious that a these features are prone to change after bariatric surgery, but what happens with the functional and structural parameters of patients who do not have left ventricular hypertrophy (LVH) and/or hypertension. Comparative studies between these patient groups can give us direction in different features of cardiac remodelling after bariatric surgery.

Also a research field that needs to be investigated is the influence of bariatric surgery cardiac rhythm disorders. It is well known that weight loss has a positive effect on the treatment of rhythm disorders such atrial fibrillation, but the literature on the effects of bariatric surgery on these rhythm disorders are sparse. $(62,63)$ Also we do not know what the coexistence of features like LVH/RVH and (pulmonary) hypertension does on treatability of rhythm disorders in obese patients. The effects of bariatric surgery on cardiac physiology need to be investigated in more specific patient subgroups to determine adequate benefits.

Regarding pulmonary physiology in the obese and after bariatric surgery, more research is showing at we need to determine adequate subgroups prone for postoperative complications. In chapter 6 was stated that the maximum inspiratory pressure does not have an influence on postoperative complications in bariatric surgery. In recent studies by Van Huisstede et al. $(4,5,64)$ the interaction between pulmonary diseases, pulmonary physiology, obesity and bariatric surgery were investigated. One of the main findings of these studies was that patients with abnormal spirometry; an $\mathrm{FEV}_{1} / \mathrm{FVC}<70 \%$ and a $\delta \mathrm{FEV}_{1} \geq 12 \%$ have an increased risk of postoperative complications. (64) With these identified groups, future research can focus on training these specific patient populations with the goal to decrease this risk of postoperative complications. Secondly we need to look at the burden of comorbidities and the risk of postoperative complications. Patients with more comorbidities (e.g. heart failure, COPD) have an increased risk of postoperative complications compared to patients with one comorbidity. This enables researchers to look at the interaction between different comorbidities and its relationship with the postoperative period.

Finally, the remission of T2DM will be point of discussion in the next few years. Basically which surgical procedure is the most suitable for patients with T2DM. Chapter 11 showed 
that in terms of glycemic control the Diverted Sleeve Gastrectomy with Ileal Transposition (DSIT) is superior compared with the Mini Gastric Bypass (MGB) and the Sleeve Gastrectomy (SG) in the first 30 days after surgery. These improvements in glycemic control after DSIT find their basis in the Forgut-Hindgut hypothesis. (52-54) By transferring the distal ileum just below the pylorus, digested food stimulates the production of satiety hormones like GLP-1, PYY and oxyntomodullin. (54) These hormones are also known for their anti-diabetogenic properties and also increased blood levels are found after other surgical procedures, like the Roux en-Y Gastric Bypass (RYGB), Laparoscopic Adjustable Gastric Banding (LAGB) and Biliopancreatic Diversion with or without duodenal switch (BPD-DS). However in current literature, there is lack of studies comparing surgical models and conventional models in terms of production of these hormones. Therefore Celik et al. (66) recently started with the HIPER-1 project to investigate the production of hormones GLP-1 and PYY in different surgical and non-surgical models.

In conclusion, as stated by Cummings and Cohen (57), we are ready for a change in our current guidelines, mainly to broaden the indications for bariatric and metabolic surgery. (57) With a landmark study like the Swedish Obese Subjects study (that has generated the foremost evidence base in the speciality) we were able to change current guidelines, but still the indications for bariatric and metabolic surgery need to be broadened. Therefore the challenges lie in designing high-quality randomised controlled trials and to design population based studies by combining national and international databases. Eventually this will lead to new guidelines and indications for bariatric and metabolic surgery. 


\section{REFERENCES}

1. Pories WJ, MacDonald KG, Jr., Flickinger EG, Dohm GL, Sinha MK, Barakat HA, et al. Is type II diabetes mellitus (NIDDM) a surgical disease? Annals of surgery. 1992;215(6):633-42; discussion 43.

2. Pories WJ, Swanson MS, MacDonald KG, Long SB, Morris PG, Brown BM, et al. Who would have thought it? An operation proves to be the most effective therapy for adult-onset diabetes mellitus. Annals of surgery. 1995;222(3):339-50; discussion 50-2.

3. Buchwald H, Estok R, Fahrbach K, Banel D, Jensen MD, Pories WJ, et al. Weight and type 2 diabetes after bariatric surgery: systematic review and meta-analysis. The American journal of medicine. 2009;122(3):248-56 e5.

4. van Huisstede A, Rudolphus A, Castro Cabezas M, Biter LU, van de Geijn GJ, Taube C, et al. Effect of bariatric surgery on asthma control, lung function and bronchial and systemic inflammation in morbidly obese subjects with asthma. Thorax. 2015;70(7):659-67.

5. van Huisstede A, Rudolphus A, van Schadewijk A, Cabezas MC, Mannaerts GH, Taube C, et al. Bronchial and systemic inflammation in morbidly obese subjects with asthma: a biopsy study. American journal of respiratory and critical care medicine. 2014;190(8):951-4.

6. Jeng EI, Aranda JM, Jr., Ahmed M, Klodell CT. Left Ventricular Assist Device and Bariatric Surgery: A Bridge to Heart Transplant by Weight and Waiting Time Reduction. Journal of cardiac surgery. 2016;31(2):120-2.

7. Lim CP, Fisher OM, Falkenback D, Boyd D, Hayward CS, Keogh A, et al. Bariatric Surgery Provides a "Bridge to Transplant" for Morbidly Obese Patients with Advanced Heart Failure and May Obviate the Need for Transplantation. Obesity surgery. 2016;26(3):486-93.

8. Aggarwal R, Harling L, Efthimiou E, Darzi A, Athanasiou T, Ashrafian H. The Effects of Bariatric Surgery on Cardiac Structure and Function: a Systematic Review of Cardiac Imaging Outcomes. Obesity surgery. 2015.

9. Fried M, Yumuk V, Oppert JM, Scopinaro N, Torres A, Weiner R, et al. Interdisciplinary European guidelines on metabolic and bariatric surgery. Obesity surgery. 2014;24(1):42-55.

10. Chaudhry UI, Kanji A, Sai-Sudhakar CB, Higgins RS, Needleman BJ. Laparoscopic sleeve gastrectomy in morbidly obese patients with end-stage heart failure and left ventricular assist device: medium-term results. Surgery for obesity and related diseases : official journal of the American Society for Bariatric Surgery. 2015;11(1):88-93.

11. McCloskey CA, Ramani GV, Mathier MA, Schauer PR, Eid GM, Mattar SG, et al. Bariatric surgery improves cardiac function in morbidly obese patients with severe cardiomyopathy. Surgery for obesity and related diseases : official journal of the American Society for Bariatric Surgery. 2007;3(5):503-7.

12. Ramani GV, McCloskey C, Ramanathan RC, Mathier MA. Safety and efficacy of bariatric surgery in morbidly obese patients with severe systolic heart failure. Clinical cardiology. 2008;31(11):51620 . 
13. Lim CP, Fisher OM, Falkenback D, Boyd D, Hayward CS, Keogh A, et al. Bariatric Surgery Provides a "Bridge to Transplant" for Morbidly Obese Patients with Advanced Heart Failure and May Obviate the Need for Transplantation. Obesity surgery. 2015.

14. Samaras K, Connolly SM, Lord RV, Macdonald P, Hayward CS. Take heart: bariatric surgery in obese patients with severe heart failure. Two case reports. Heart, lung \& circulation. 2012;21(12):847-9.

15. Ristow B, Rabkin J, Haeusslein E. Improvement in dilated cardiomyopathy after bariatric surgery. Journal of cardiac failure. 2008;14(3):198-202.

16. Ashrafian H, le Roux CW, Darzi A, Athanasiou T. Effects of bariatric surgery on cardiovascular function. Circulation. 2008;118(20):2091-102.

17. Ashrafian H, Athanasiou T, le Roux CW. Heart remodelling and obesity: the complexities and variation of cardiac geometry. Heart (British Cardiac Society). 2011;97(3):171-2.

18. Vest AR, Heneghan HM, Agarwal S, Schauer PR, Young JB. Bariatric surgery and cardiovascular outcomes: a systematic review. Heart (British Cardiac Society). 2012;98(24):1763-77.

19. Garza CA, Pellikka PA, Somers VK, Sarr MG, Collazo-Clavell ML, Korenfeld Y, et al. Structural and functional changes in left and right ventricles after major weight loss following bariatric surgery for morbid obesity. The American journal of cardiology. 2010;105(4):550-6.

20. Buchwald H, Avidor Y, Braunwald E, Jensen MD, Pories W, Fahrbach K, et al. Bariatric surgery: a systematic review and meta-analysis. Jama. 2004;292(14):1724-37.

21. Pickering TG, Hall JE, Appel LJ, Falkner BE, Graves J, Hill MN, et al. Recommendations for blood pressure measurement in humans and experimental animals: Part 1: blood pressure measurement in humans: a statement for professionals from the Subcommittee of Professional and Public Education of the American Heart Association Council on High Blood Pressure Research. Hypertension. 2005;45(1):142-61.

22. Maxwell MH, Waks AU, Schroth PC, Karam M, Dornfeld LP. Error in blood-pressure measurement due to incorrect cuff size in obese patients. Lancet (London, England). 1982;2(8288):33-6.

23. Linfors EW, Feussner JR, Blessing CL, Starmer CF, Neelon FA, McKee PA. Spurious hypertension in the obese patient. Effect of sphygmomanometer cuff size on prevalence of hypertension. Archives of internal medicine. 1984;144(7):1482-5.

24. Marks LA, Groch A. Optimizing cuff width for noninvasive measurement of blood pressure. Blood pressure monitoring. 2000;5(3):153-8.

25. Lindauer B, Steurer MP, Muller MK, Dullenkopf A. Anesthetic management of patients undergoing bariatric surgery: two year experience in a single institution in Switzerland. BMC anesthesiology. 2014;14:125.

26. O’Brien E, Atkins N, Stergiou G, Karpettas N, Parati G, Asmar R, et al. European Society of Hypertension International Protocol revision 2010 for the validation of blood pressure measuring devices in adults. Blood pressure monitoring. 2010;15(1):23-38.

27. O’Brien E, Pickering T, Asmar R, Myers M, Parati G, Staessen J, et al. Working Group on Blood Pressure Monitoring of the European Society of Hypertension International Protocol for validation of blood pressure measuring devices in adults. Blood pressure monitoring. 2002;7(1):317. 
28. de Senarclens O, Feihl F, Giusti V, Engelberger RP, Rodieux F, Gomez P, et al. Brachial or wrist blood pressure in obese patients: which is the best? Blood pressure monitoring. 2008;13(3):14951.

29. de Wilde RB, de Wit F, Geerts BF, van Vliet AL, Aarts LP, Vuyk J, et al. Non-invasive continuous arterial pressure and pulse pressure variation measured with $\operatorname{Nexfin}((\mathrm{R}))$ in patients following major upper abdominal surgery: a comparative study. Anaesthesia. 2016;71(7):788-97.

30. Alpert MA. Cardiac morphology and performance in severe obesity: size really does matter. Cardiology. 2014;127(3):141-3.

31. Watson RA, Pride NB, Thomas EL, Ind PW, Bell JD. Relation between trunk fat volume and reduction of total lung capacity in obese men. Journal of applied physiology (Bethesda, Md : 1985). 2012;112(1):118-26.

32. Sood A. Altered resting and exercise respiratory physiology in obesity. Clinics in chest medicine. 2009;30(3):445-54, vii.

33. Pedoto A. Lung physiology and obesity: anesthetic implications for thoracic procedures. Anesthesiology research and practice. 2012;2012:154208.

34. Hulzebos EH, Helders PJ, Favie NJ, De Bie RA, Brutel de la Riviere A, Van Meeteren NL. Preoperative intensive inspiratory muscle training to prevent postoperative pulmonary complications in high-risk patients undergoing CABG surgery: a randomized clinical trial. JAMA. 2006;296(15):1851-7.

35. Hulzebos EH, Smit Y, Helders PP, van Meeteren NL. Preoperative physical therapy for elective cardiac surgery patients. The Cochrane database of systematic reviews. 2012;11:CD010118.

36. Hulzebos EH, van Meeteren NL, van den Buijs BJ, de Bie RA, Brutel de la Riviere A, Helders PJ. Feasibility of preoperative inspiratory muscle training in patients undergoing coronary artery bypass surgery with a high risk of postoperative pulmonary complications: a randomized controlled pilot study. Clinical rehabilitation. 2006;20(11):949-59.

37. Valkenet K, de Heer F, Backx FJ, Trappenburg JC, Hulzebos EH, Kwant S, et al. Effect of inspiratory muscle training before cardiac surgery in routine care. Physical therapy. 2013;93(5):611-9.

38. Wei YF, Wu HD. Candidates for Bariatric Surgery: Morbidly Obese Patients with Pulmonary Dysfunction. Journal Of Obesity. 2012.

39. Parreira VF, Matos CMP, Athayde FTS, Moraes KS, Barbosa KS, Britto RR. Evolution of respiratory muscle strength in post-operative gastroplasty. Rev Bras Fisioter. 2012;16(3):225-30.

40. Jubber AS. Respiratory complications of obesity. Journal of Clinical Practice. 2004;58(6):573-80.

41. Magnani KL, Cataneo AJM. Respiratory muscle strength in obese individuals and influence of upper-body fat distribution. Sao Paulo Med J. 2007;125(4):215-9.

42. Weiner P, Waizman J, Weiner M, Rabner M, Magadle R, Zamir D. Influence of excessive weight loss after gastroplasty for morbid obesity on respiratory muscle performance. Thorax. 1998;53(1):39-42.

43. Berggren JR, Boyle KE, Chapman WH, Houmard JA. Skeletal muscle lipid oxidation and obesity: influence of weight loss and exercise. American journal of physiology Endocrinology and metabolism. 2008;294(4):E726-32. 
44. Ng M, Fleming T, Robinson M, Thomson B, Graetz N, Margono C, et al. Global, regional, and national prevalence of overweight and obesity in children and adults during 1980-2013: a systematic analysis for the Global Burden of Disease Study 2013. Lancet (London, England). 2014;384(9945):766-81.

45. Pouwels S, Fiddelaers J, Teijink JA, Woorst JF, Siebenga J, Smeenk FW. Preoperative exercise therapy in lung surgery patients: A systematic review. Respiratory medicine. 2015;109(12):1495504.

46. Pouwels S, Stokmans RA, Willigendael EM, Nienhuijs SW, Rosman C, van Ramshorst B, et al. Preoperative exercise therapy for elective major abdominal surgery: a systematic review. International journal of surgery (London, England). 2014;12(2):134-40.

47. Pouwels S, Willigendael EM, van Sambeek MR, Nienhuijs SW, Cuypers PW, TeijinkJA. Beneficial Effects of Pre-operative Exercise Therapy in Patients with an Abdominal Aortic Aneurysm: A Systematic Review. European journal of vascular and endovascular surgery : the official journal of the European Society for Vascular Surgery. 2015;49(1):66-76.

48. Pouwels S, Wit M, Teijink JA, Nienhuijs SW. Aspects of Exercise before or after Bariatric Surgery: A Systematic Review. Obes Facts. 2015;8(2):132-46.

49. Donadelli SP, Junqueira-Franco MV, de Mattos Donadelli CA, Salgado W, Jr., Ceneviva R, Marchini JS, et al. Daily vitamin supplementation and hypovitaminosis after obesity surgery. Nutrition (Burbank, Los Angeles County, Calif). 2012;28(4):391-6.

50. Gasteyger C, Suter M, Gaillard RC, Giusti V. Nutritional deficiencies after Roux-en-Y gastric bypass for morbid obesity often cannot be prevented by standard multivitamin supplementation. The American journal of clinical nutrition. 2008;87(5):1128-33.

51. Verger EO, Aron-Wisnewsky J, Dao MC, Kayser BD, Oppert JM, Bouillot JL, et al. Micronutrient and Protein Deficiencies After Gastric Bypass and Sleeve Gastrectomy: a 1-year Follow-up. Obesity surgery. 2016;26(4):785-96.

52. Celik A, Asci M, Celik BO, Ugale S. The impact of laparoscopic diverted sleeve gastrectomy with ileal transposition (DSIT) on short term diabetic medication costs. SpringerPlus. 2015;4:417.

53. Celik A, Ugale S, Ofluoglu H, Asci M, Celik BO, Vural E, et al. Technical feasibility and safety profile of laparoscopic diverted sleeve gastrectomy with ileal transposition (DSIT). Obesity surgery. 2015;25(7):1184-90.

54. Celik A, Ugale S, Ofluoglu H, Vural E, Cagiltay E, Cat H, et al. Metabolic Outcomes of Laparoscopic Diverted Sleeve Gastrectomy with Ileal Transposition (DSIT) in Obese Type 2 Diabetic Patients. Obesity surgery. 2015;25(11):2018-22.

55. Kular KS, Manchanda N, Cheema GK. Seven Years of Mini-Gastric Bypass in Type II Diabetes Patients with a Body Mass Index <35 kg/m. Obesity surgery. 2015.

56. Panunzi S, De Gaetano A, Carnicelli A, Mingrone G. Predictors of remission of diabetes mellitus in severely obese individuals undergoing bariatric surgery: do BMI or procedure choice matter? A meta-analysis. Annals of surgery. 2015;261(3):459-67.

57. Cummings DE, Cohen RV. Beyond BMI: the need for new guidelines governing the use of bariatric and metabolic surgery. The lancet Diabetes \& endocrinology. 2014;2(2):175-81. 
58. Flum DR, Belle SH, King WC, Wahed AS, Berk P, Chapman W, et al. Perioperative safety in the longitudinal assessment of bariatric surgery. The New England journal of medicine. 2009;361(5):445-54.

59. Nguyen NT, Hinojosa M, Fayad C, Varela E, Wilson SE. Use and outcomes of laparoscopic versus open gastric bypass at academic medical centers. Journal of the American College of Surgeons. 2007;205(2):248-55.

60. Batterham RL, Cummings DE. Mechanisms of Diabetes Improvement Following Bariatric/ Metabolic Surgery. Diabetes care. 2016;39(6):893-901.

61. Patel R, Lim RP, Saric M, Nayar A, Babb J, Ettel M, et al. Diagnostic Performance of Cardiac Magnetic Resonance Imaging and Echocardiography in Evaluation of Cardiac and Paracardiac Masses. The American journal of cardiology. 2016;117(1):135-40.

62. Shoemaker MB, Gidfar S, Pipilas DC, Tamboli RA, Savio Galimberti E, Williams DB, et al. Prevalence and predictors of atrial fibrillation among patients undergoing bariatric surgery. Obesity surgery. 2014;24(4):611-6.

63. Lemery R. Heart rate and rhythm benefits from bariatric surgery, weight loss, and associated matters: another autonomic tale. Heart rhythm : the official journal of the Heart Rhythm Society. 2011;8(1):91-2.

64. van Huisstede A, Biter LU, Luitwieler R, Castro Cabezas M, Mannaerts G, Birnie E, et al. Pulmonary function testing and complications of laparoscopic bariatric surgery. Obesity surgery. 2013;23(10):1596-603.

65. Zhu J, Gupta R, Safwa M. The Mechanism of Metabolic Surgery: Gastric Center Hypothesis. Obesity surgery. 2016;26(7):1639-41.

66. Celik A, Dixon JB, Pouwels S, Celik BO, Karaca FC, Gupta A, et al. Effects of different metabolic states and surgical models on glucose metabolism and secretion of ileal L-cell peptides: protocol for a cross-sectional study. BMJ open. 2016;6(3):e010245. 

14. VALORISATION 

Since the mid-twentieth century, the influence of surgical procedure into metabolic disease became clearer. Starting with a report of Friedman and colleagues wherein they demonstrated in 1955 that a gastrectomy resulted in resolution of type 2 diabetes mellitus (T2DM). In the 70 's and 80's, Bosello and colleagues demonstrated the key mechanisms and interplay between morbid obesity and T2DM. They observed that after a jejunoileal bypass weight loss was the primary factor in lowering hyperglycemia and decreasing insulinemia. Ackerman and Halverson found that immediately after malabsorptive bariatric surgical procedures T2DM patients became euglycemic and were freed from diabetes medication.

Nowadays, with the obesity pandemic reaching epic proportions, there is an ever-increasing need for change. More than 30\% of the world population has obesity and associated comorbidities. Scientific research of the last decade has shown that bariatric surgery has effect on metabolic diseases apart from the impact of weight loss. For this reason several bariatric organisations have changed their names into societies for 'bariatric and metabolic surgery'.

\section{RELEVANCE OF THE SCIENTIFIC RESULTS IN THIS THESIS}

This thesis contains two parts, both with the purpose of understanding perioperative physiology in bariatric and metabolic surgery. The first part describes cardiopulmonary physiology before and after bariatric and metabolic surgery. Regarding cardiac physiology, specifically cardiac structure and function, patients with pre-existent left ventricular hypertrophy (LVH) and/or hypertension benefit the most from bariatric surgery. According to the available literature, bariatric and metabolic surgery will induce short-term cardiac remodelling in these patients (chapter 2).

Several methods are available to assess cardiovascular hemodynamics (e.g. blood pressure (BP)). One example is the intra-arterial BP measurement, mostly measured of the radial artery, as considered the most accurate one. Because of its invasiveness and its risk for infection it is not suitable for BP measurement in obese patients scheduled for bariatric surgery. Another measurement tool is the auscultatory sphygmomanometry, widely used in clinical practice, based on the principles of Riva-Rocci/Korotkoff. However these measurements can give an overestimation of the BP (when using the correct blood pressure cuff). The measurement device used in this thesis is the Nexfin ${ }^{\circledR}$. This device continuously measures the pressure waveform in the finger and calculates the beat-to-beat brachial BP through an algorithm. The Nexfin ${ }^{\otimes}$ device was used in the bariatric population to determine its relation with Riva-Rocci/ Korotkoff BP measurement (chapter 3) and to assess cardiovascular hemodynamics before and after bariatric and metabolic surgery (chapter 4 ). The Nexfin ${ }^{\circledR}$ device appeared to be easy to use and capable to determine a trend in cardiovascular hemodynamics. It is less dependent 
on brachial blood pressure variations in relation to the circumference of the upper arm and cuff size. Therefore the Nexfin ${ }^{\circledR}$ device can be easily used for clinical and research purposes in obese individuals.

Regarding pulmonary physiology chapters 5 and 6 showed that the altered pulmonary function as a result of obesity could give potential challenges in perioperative care. Changes in pulmonary function are not solely a change in muscle function, but are interplay between muscle function, intrinsic function of the lungs and thoracic compliance (chapter 5 and 6). However predicting inspiratory muscle function might not be useful in bariatric surgery, in other specialties (such as cardiac surgery) it is standard practice (chapter 7).

The final chapter of the first part of this thesis demonstrates the integrative aspect of cardiopulmonary physiology in perioperative exercise. In chapter 8 is shown that perioperative exercise in bariatric surgery is beneficial in terms of weight loss and the reduction of cardiovascular risk factors. However, the optimal exercise regimen for which patient remains unclear. Based on the results found, we recommended an exercise regimen ideally pre- and postoperative. These exercise regimens should last for a median of 12 weeks, has an intensity of either $65 \%$ peak heart rate or $65 \%$ VO2 max and is at least partially supervised. When implemented these exercise regimens should give additional benefit for patients before and after bariatric surgery.

The second part of this thesis focussed on two aspects of metabolic physiology; vitamin deficiencies and type 2 diabetes mellitus (T2DM) remission. Screening for nutritional deficiencies is costly. Trying to reduce the costs, an algorithm was developed (Chapter 9), to improve and optimize the screening for nutrient deficiencies after bariatric surgery. We have found that our algorithm is able to reduce vitamin D, B12, B6, B1 and ferritin examinations by $15 \%, 11 \%, 28 \%, 28 \%$ and $38 \%$, respectively, without missing clinically relevant deficiencies. Also this corresponds with a potential cost savings of $14 \%$.

The supplementation regimen of vitamin B12 deficiencies after bariatric and metabolic surgery was the focus of chapters 10 and 11 . The literature review in chapter 10 showed that to treat a vitamin B12 deficiency with oral multivitamin supplements, a minimum dosage of 350 microgram was necessary. Oral supplements with lower dosages were not able to recover vitamin B12 deficiencies biochemically. Chapter 11 showed us that in a retrospective study a 6 intramuscular vitamin B12 injection regime was able to recover all vitamin B12 deficiencies. According to these two studies an oral supplement must have at least 350 microgram of vitamin B12. Secondly, an intravenous supplementation regimen should at least contain 6 intramuscular injections with a loading dose (one injection). Each injection should contain $1000 \mu \mathrm{g}$ of hydroxocobalamin. 
In the final chapter of part II (chapter 12) we have assessed the time to glycemic control after several bariatric surgical procedures. T2DM is a major burden and therefore the faster remission is achieved, the better it is for patients but also it reduces healthcare costs significantly. Our results demonstrated that in the first 30 days after surgery, patients who had a Diverted Sleeve Gastrectomy and Ileal Transposition (DSIT), or a Mini Gastric Bypass (MGB) achieved glycemic control after respectively 29 and 30 days.

\section{POPULATION OF INTEREST}

The results of this thesis are relevant for several groups of patients. Firstly, patients who were recently operated or patients who want to undergo a bariatric surgical procedure. Secondly, this thesis emphasises that bariatric surgery might be beneficial for more than just weight loss. Patients with metabolic, cardiac and/or pulmonary diseases can benefit from bariatric/ metabolic surgery.

This thesis is also of interest of clinicians who treat patients with therapeutic resistant cardiac, pulmonary of metabolic diseases. Also, the contents of this thesis are of interest for expert panels, responsible for the development of national and international guidelines.

\section{INNOVATION AND FUTURE}

The impact of bariatric surgery on cardiac, pulmonary and metabolic diseases apart from its weight loss effect is becoming clearer. This is not yet reflected by the current guidelines regarding bariatric surgery.

The guidelines of the International Federation of Surgery for Obesity and Metabolic disorders (IFSO) state that surgery is warranted to patients with a BMI greater than $40 \mathrm{~kg} / \mathrm{m}^{2}$, or greater than $35 \mathrm{~kg} / \mathrm{m}^{2}$ with serious comorbidities such as type 2 diabetes mellitus. These guidelines were carefully written at that time, but nowadays these guidelines have increasing limitations. Among these limitations are that there were only moderate recommendations for diabetes and metabolic disorders, and that these guidelines are based on only open operations, whereas most procedures are now undertaken laparoscopically, which is safer with a ten-times lower operative mortality. One of the most important goals for future research is elucidating the mechanisms responsible for the remission of metabolic diseases. We have to gain more insight in the effects of bariatric and metabolic surgery on the remission of cardiac and pulmonary diseases. And so, we can help more patients with metabolic diseases. 


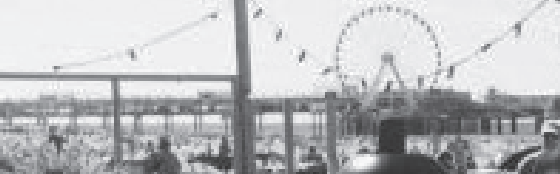

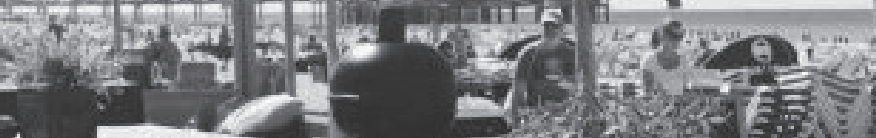

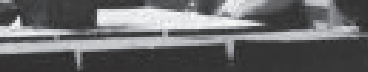

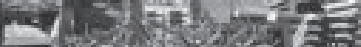

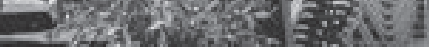

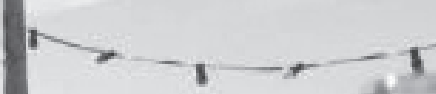

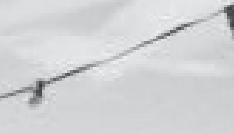

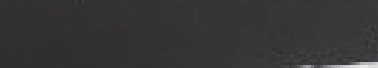

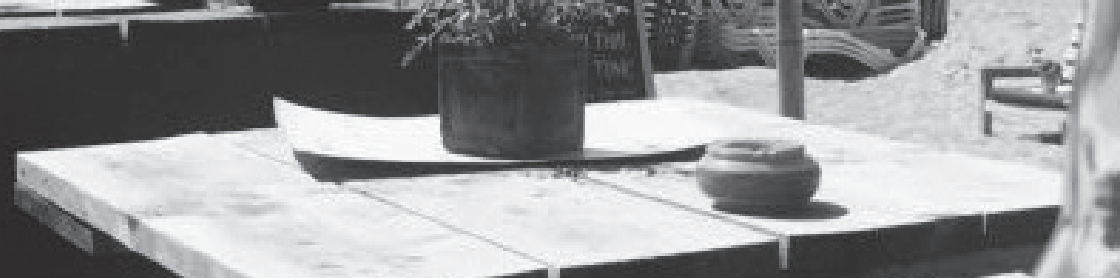

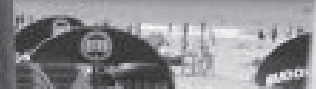

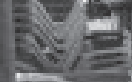

(2)

$$
3 .
$$
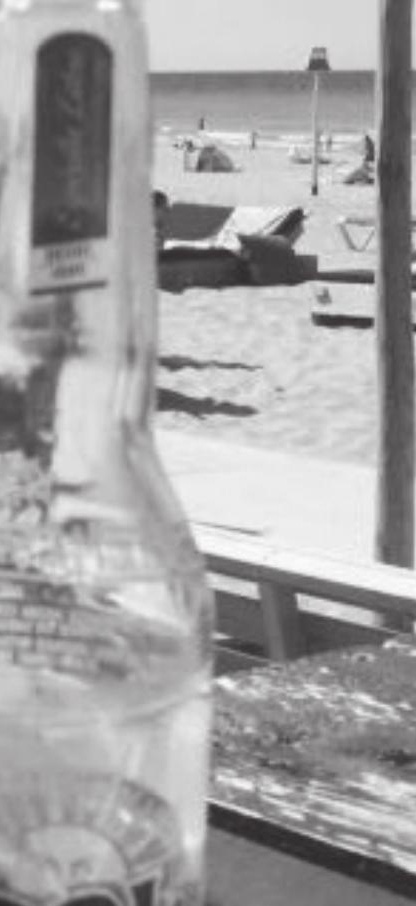

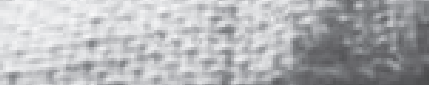

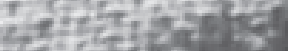

$\left.x_{1}+x_{1}\right)^{2}=$

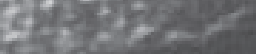

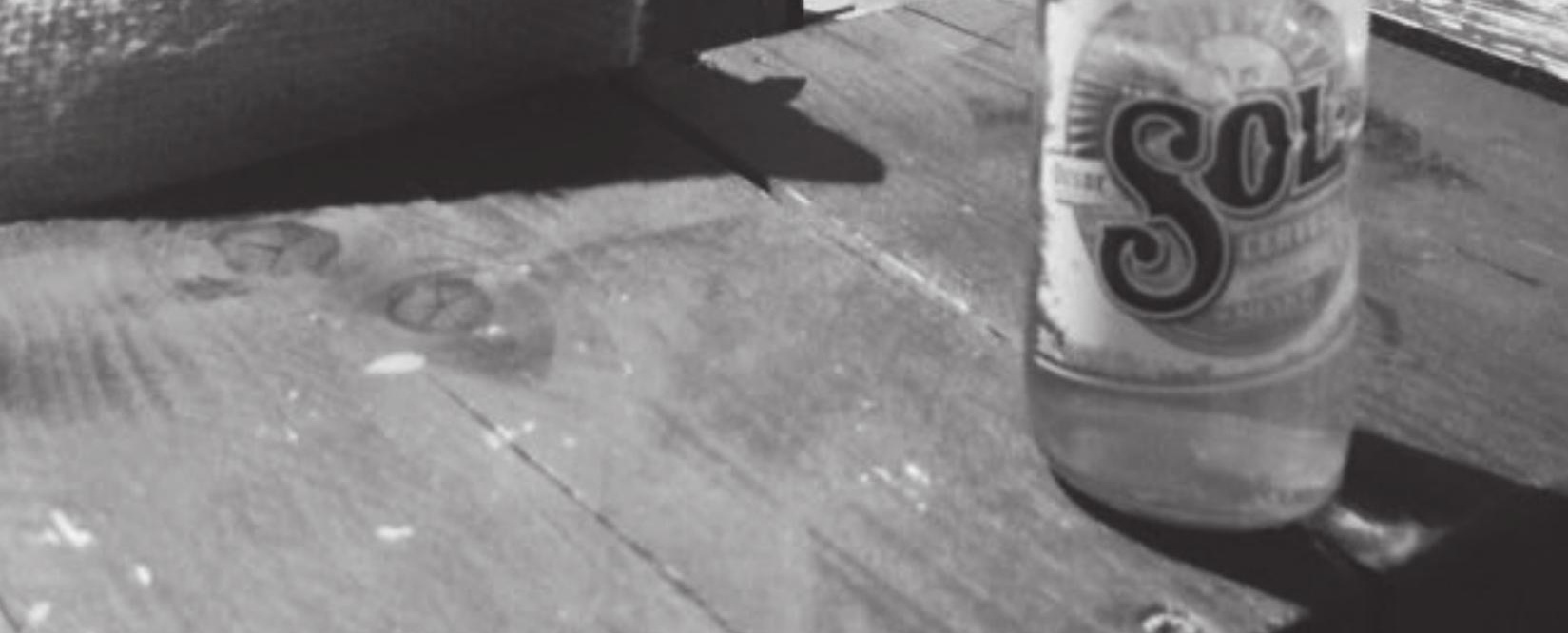


15. NEDERLANDSE SAMENVATTING 

In dit proefschrift worden twee domeinen van de perioperatieve fysiologie in de bariatrische en metabole chirurgie besproken. Het eerste onderwerp is de cardiale en pulmonale fysiologie. In het tweede onderwerp de metabole fysiologie, met een nadruk op de vitamine en mineralen suppletie en de remissie van type 2 diabetes mellitus na bariatrische en metabole chirurgie. In beide domeinen worden tevens optimalisatie strategieën besproken.

In hoofdstuk 2 wordt een overzicht gegeven van de literatuur met betrekking tot de verandering in cardiale functie en hemodynamiek voor en na bariatrische chirurgie. Hieruit blijkt dat bariatrische chirurgie gunstige effecten heeft op de functie van het hart. Teven blijkt dat patiënten met pre-existente hypertensie dan wel linker ventrikel hypertrofie het meeste baat hebben bij bariatrische en metabole chirurgie op basis van de huidige literatuur.

Hoofdstuk 3 beschrijft de resultaten van de validatiestudie van het Nexfin ${ }^{\circledast}$ hemodynamiek monitoring apparaat, afgezet tegen de reguliere bloeddrukmeting bij obese patienten voorafgaand aan bariatrische chirurgie. Hoofdstuk 4 omschrijft de verandering in de hemodynamiek voor en na bariatrische chirurgie gemeten middels de

Nexfin ${ }^{\circledR}$. Het blijkt dat na bariatrische en metabole chirurgie er een significante daling optreedt van de systolische en de diastolische bloeddruk en tevens het slagvolume. Ook heet bariatrische en metabole chirurgie gunstige effecten op de cardiac output.

Een overzicht van de invloeden van obesitas op de pulmonale fysiologie en zijn implicaties voor de klinische praktijk worden gegeven in hoofdstuk 5. In hoofdstuk 6 worden de verandering van de maximale inspiratoire druk beschreven voor en na bariatrische chirurgie. In de eerste drie maanden postoperatief treedt er een significante daling op in maximale inspiratoire druk, maar na negen maanden stijgt de maximale inspiratoire druk wordt er een stijgend trend waargenomen, waarschijnlijk het gevolg van het 'wennen' aan de nieuwe lichaamssituatie. Hoofdstuk 7 is een logisch gevolg en kijkt derhalve of de verandering in maximale inspiratoire druk bij obese patiënten voorspeld kan worden aan de hand van voorspelformules. Hoofdstuk 8 geeft een kritisch overzicht over de meerwaarde van perioperatieve bewegingsinterventies bij bariatrische en metabole chirurgie.

Hoofdstuk 9 is het eerste hoofdstuk in het gedeelte 'metabole fysiologie' en beschrijft de variaties in suppletie strategieën van patiënten met een vitamine B12 deficiëntie. De retrospectieve studie in Hoofdstuk 10 onderzocht de effecten van drie verschillende vitamine B12 suppletie regimes. In hoofdstuk 11 wordt een algoritme beschreven om de screening naar vitamine en mineralen deficiënties voor en na bariatrische chirurgie te optimaliseren en het blijkt dat dit algoritme een significant kostenreductie teweeg kan brengen. Het laatste hoofdstuk (hoofdstuk 12) van dit proefschrift heeft onderzocht welke van de drie typen 
bariatrisch chirurgische operaties het snelste kan zorgen voor het in remissie treden van diabetes mellitus type 2 in de eerste 30 dagen postoperatief. Hieruit blijkt dat de 'diverted sleeve gastrectomy and ileal transposition (DSIT)' mogelijk superieur is aan de 'mini gastric bypass (MGB)' en de 'sleeve gastrectomy (SG).' 

16. DANKWOORD 

And we are done...

Een promotieonderzoek, dat klinkt heel simpel en eenvoudig, maar niets is minder waar. Het is de ultieme beproeving op wetenschappelijk, psychisch en lichamelijk niveau. Gedurende een periode van een aantal jaar deel je lief en leed, ergernis, depressies en vreugde momenten met een selecte groep mensen, waarvan een ieder direct of indirect een steentje heeft bijgedragen. Die mensen wil ik heel graag bedanken.

\section{Beste prof. dr. Teijink, beste Joep,}

Ik weet het nog als de dag van gisteren. Het was een bewolkte donderdagmiddag (zoals eigenlijk iedere donderdag in de winter in Nederland) en ik zat bij jou op kantoor, toen je de basis schetste van mijn proefschrift. Ik als kersverse, maar gemotiveerde coassistent destijds, was zwaar onder de indruk van deze voortvarendheid. In de loop der jaren zijn ongeveer tien plannen voor potentiele proefschriften voorbijgekomen, maar eentje was genoeg. Ik wil je hartelijk danken voor je vertrouwen en je kritische doch rechtvaardige commentaren op de stukken die wij in de loop der jaren hebben geschreven. Ik heb veel bewondering voor je motivatie en veelzijdigheid, hoe je extreem veel balletjes in de lucht kan houden. Daarnaast de vele andere hoogtepunten waarvan ons ESVS tripje naar Porto wel de leukste was en waar we menig discotheek op zijn kop hebben gezet...

\section{Beste dr. Nienhuijs, beste Simon,}

Het lijkt de ideale combinatie een promotor die qua plannen en ideeën niet te stoppen is en een copromotor die alle zaken rustig bekijkt en op waarde weet te schatten. In de afgelopen jaren heb ik veel klinische en wetenschappelijke zaken van je mogen leren, maar ook kwamen we erachter dat onze muzieksmaken niet heel veel van elkaar verschillen. Jammer dat we voor 'I Love Techno' nu helemaal naar Zuid-Frankrijk moeten (in plaats van Gent), maar goed dat mag de pret niet drukken.

\section{Beste dr. Buise, beste Marc,}

Toen mijn proefschrift meer richting perioperatieve fysiologie ging, kwam jij als anesthesioloog om de hoek kijken. Dank voor je kritische commentaar en je altijd heldere uitleg met betrekking tot de cardiopulmonale fysiologie en de invloeden van obesitas. Daarnaast wil ik je ook bedanken voor je vertrouwen en alles wat ik van je heb mogen leren op de Intensive Care. 


\section{Beste dr. Ingels en dr. Beurskens, beste Koen en Carien,}

Weliswaar inhoudelijk niets te maken gehad met mijn proefschrift, maar wel met mijn vorming als wetenschappelijk-onderzoeker. Onder jullie vakkundige en kritische leiding heb ik mijn wetenschappelijke basis gelegd en met een mooi resultaat. Wie had ooit gedacht dat we met een ogenschijnlijk simpel idee, de basis zouden leggen voor een complete onderzoeksgroep, met nu al drie promovendi. Zonder jullie had mijn proefschrift en wetenschappelijke ontwikkeling er heel anders uitgezien. Ik zeg, op naar Los Angeles!

\section{Beste dr. Willigendael, beste Edith,}

Vanaf het begin dat wij elkaar kennen, betitelde jij jezelf als 'onderzoeksmama'. Niets is minder waar. Edith, ik wil je hartelijke bedanken voor de goede samenwerking, vriendschap en voor alle wijze raad in goede tijden, maar ook in de mindere tijden die ik in de afgelopen jaren gekend heb.

Prof. dr. Bouvy, prof. dr. Buhre, prof. dr. Rosman en prof. dr. Scharnhorst, leden van de beoordelingscommissie. Hartelijk dank voor het aanvaarden van deze positie, en voor jullie kritische beoordeling van dit proefschrift.

Graag wil ik alle medeauteurs bedanken voor hun waardevolle bijdragen en commentaren. In het bijzonder veel dank aan prof. dr. Frank Smeenk, voor alle vakkundige ondersteuning bij de pulmonaal georiënteerde stukken. Ook als opleider heb ik veel van je geleerd en jij bent degene geweest die me mee heeft genomen naar de wereld 'achter het artikel', anders gezegd hoe moet een lezer jouw artikel lezen en wat voor boodschap je wil uitdragen. Ook hoop ik dat ons gezamenlijke project met de Cliniques Universitaire Saint-Luc in Brussel mooie resultaten gaat opleveren. Daarnaast speciale dank aan dr. Arthur Bouwman. Ik heb nog nooit iemand gezien die artikelen zo kritisch nakijkt, bestudeert en letterlijk elke steen omdraait. Voor mijn gevoel duurde het altijd een eeuwigheid voordat ik commentaar van je terugkreeg, maar altijd tot in de puntjes verzorgd en het bracht het artikel altijd naar een hoger niveau. Ook dr. Lukas Dekker en dr. Patrick Houthuizen wil ik heel hartelijk bedanken voor hun input bij de cardiologisch georiënteerde artikelen in dit proefschrift. Als laatste bijzondere waardering voor data wizard en klinische chemicus/endocrinoloog dr. Arjen-Kars Boer en de enige echte epidemioloog/ spraakwaterval dr. Saskia Houterman.

\section{Arts-assistenten en arts-onderzoekers Heelkunde/IC van het CZE}

De afgelopen jaren heb ik met veel plezier met jullie mogen samenwerken en natuurlijk mogen feestvieren. In het bijzonder wil ik mijn bariatrie broeder Martin van Wezenbeek bedanken. Weet zeker dat de lokale Weense bevolking nog lang napraat over ons bezoek aan het IFSO congres... 


\section{Chirurgen en Intensivisten van het CZE}

In de afgelopen jaren heb ik het genoegen gehad om veel van jullie te mogen leren, zowel in de kliniek als daarbuiten. Daarvoor mijn oprechte dank. In het bijzonder wil ik Frans Smulders bedanken. Van vliegende flessen wijn in een club in Wenen en diepgaande gesprekken met een berg hamburgers in een Zweedse McDonalds, tot in recordtijden een gastric sleeve doen (en in recordtijd bepaalde Franse chirurgen figuurlijk laten ontploffen), de bijnaam 'opperbaas' misstaat je absoluut niet. Ook de 'rollator PhD's' Ingeborg Herold, Marco Haanschoten en Herman Kreeftenberg verdienen een eervolle vermelding. Er is niets leukers om tussen de operaties, de SIT oproepen en het acht uur journaal, nog even de statistische ergernissen van de dag door te nemen.

\section{Mijn Franciscus SEH en Chirurgie collega's}

Toen ik de parel van Brabant verliet, had ik nooit gedacht dat het in Rotterdam minstens net zo gezellig zou zijn. Wat moet ik zonder mijn lieftallige collega's in het turbulente Rotterdam en Schiedam. Speciale dank aan de man die sneller arteriële (of veneuze) bloedgassen prikt, dan Lucky Luke op zijn schaduw schiet. En ook nog mensen red, terwijl hij zelf met een halve anafylactische shock rondloopt Chris Nijdam, wat hebben wij een aantal gekke diensten gehad. Ook KNO koning Djazz van der Heijden is zonder twijfel een echte baas, Merocel hier, Rapid Rhino daar, zelf knie reconstructies kan deze man in een handstand. Echt een waar genoegen om jullie als vrienden en collega's te hebben. \#vlietlandlife

\section{Marieke Smelt}

Mijn opvolgster. Je ziet maar zelden mensen die zo'n motivatie en drive hebben om verder te komen in hun carrière. Daarom kijk ik met veel plezier uit naar jou promotie die zeer snel zal gaan plaatsvinden.

\section{Robin, Rémondo en Mila}

Mijn Rotterdamse buurtjes (echter een paar wijken verderop). Wat is het toch altijd een plezier om jullie als vrienden te hebben. Jullie zijn het bewijs dat hard werken loont en dat bewonder ik zeer.

\section{Mijn paranimfen Ben en Sanne}

Wat een groot genoegen dat jullie tijdens mijn promotie aan mijn zijde staan. Als ex-collega's, stapmaatjes en mijn allerbeste vrienden staan jullie naast me. Ik weet het nog als de dag van gisteren, dat we in de lokale Albert Heijn te Arnhem onze handen vol hadden aan 'boefjes' die de winkel leeg probeerde te jatten. Dat waren me nog eens tijden. 


\section{Mijn 010 en 020 maatjes: Martin, Mark, Kirby, Rudi, Barry, Tamara en Marianna}

Wij hebben in de loop der jaren menig discotheek/festival/club/strandtent onveilig gemaakt en geen muziekgenre is ontsnapt aan onze visie. Van chagrijnig lopen door het Amsterdamse bos na afloop van '909' festival tot vliegen staan meppen bij Hernan Cattaneo op Loveland, wij hebben bewezen dat 'raven' topsport is. Nu alleen het Olympisch Comité nog even overtuigen.

\section{Zamir, Muhamad, Besir, Sjerionda, Erik, Marga, Bjorn, Jens en Manon}

Mijn maatjes, mijn steun en toeverlaat in donkere dagen, mijn beste vrienden. Ik wil jullie nogmaals laten blijken hoe veel ik jullie vriendschap waardeer. Zonder jullie, had ik dit promotieonderzoek niet kunnen doen. Dank voor jullie interesse, wijze raad en af en toe harde woorden die mij met beide benen op de grond hebben laten staan.

\section{Familie Elhatri,}

Elke keer is als kwam trainen na een dag hard werken, viel ik in een warm bad. Zelden zie je een familie bedrijf wat zo goed functioneert. Ieder met zijn eigen specifieke doelen en op die manier complementeren jullie elkaar. Appie, Farah, Fatima en Hayad hartelijk dank voor jullie vriendschap, gastvrijheid en jullie hebben mijn interesse voor vechtsporten nieuw leven ingeblazen in een tijd dat ik het privé erg moeilijk had. Dat heeft mij zeer goed gedaan.

\section{Johan van de Vliet,}

Johan, ik weet eigenlijk niet zo goed waar ik moet beginnen. 'Krav Maga heeft me gegrepen!' En dat is in de loop der tijd wel duidelijk geworden. Ook je wijze raad en discipline hebben mij zeer goed gedaan. En ja, wij gaan nog een keer voor een seminar naar Polen. Afgesproken?

\section{My Mexican friends Cindy and Manuel,}

We met in Philadelphia during the 'Weight Loss and Fitness Expo' and since then we became friends. It's a true honour to have you guys present at my thesis defence. It means a lot to me.

\section{Dear dr. Gupta, dear Adarsh,}

Together with Alper Çelik and a lot of our foreign colleagues we have started a research group investigating the physiological mechanisms of type 2 diabetes remission after bariatric and metabolic surgery. The result of a diner we had together in Philadelphia with pizza and cheese-steak. Thank you for your friendship and collaboration. I'll see you soon!

\section{Dear prof. dr. Çelik, dear Alper,}

As you have said it before, that meeting in Philadelphia was a game changer for us. Since then we became good friends and colleagues. Thank you for your trust, your good advice and your 
hospitality. It was really nice to visit you guys in Istanbul a few months ago and I'm looking forward to the Metabolus Istanbul meeting in 2018. Also I have noticed that Wing-Chun Kungfu and Krav Maga have a lot of similarities. Let's keep on rocking!

\section{Sjaak, Nina, Martin en Marcel}

Jullie steun is onbeschrijfelijk en eigenlijk niet in woorden uit te drukken. Daar waar mijn eigen ouders en mijn broertje me in de steek lieten, hebben jullie mij opgevangen in de meest donkere tijden van mijn korte leven. Bij jullie voel ik mij thuis, gewaardeerd, geliefd en kan ik mijzelf zijn. Mijn oprechte dank voor het feit dat jullie altijd in mij hebben geloofd en mij hebben gesteund. 


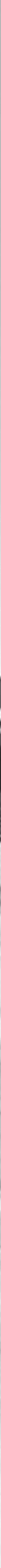


17. CURRICULUM VITAE 



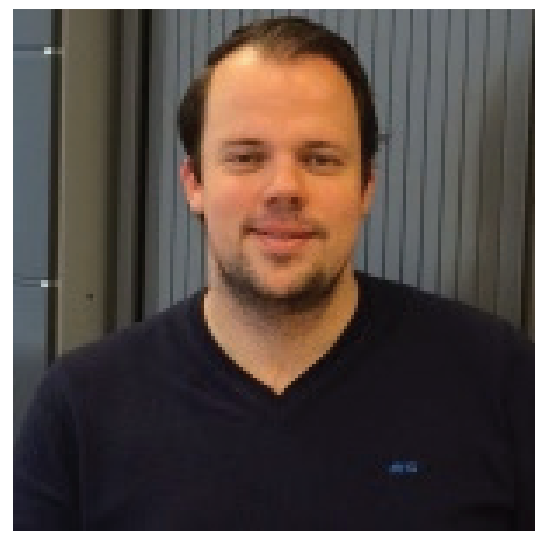

Jacobus Hendrikus (Sjaak) Pouwels werd op 11 december 1989 geboren in Amsterdam. Na het behalen van zijn Gymnasium diploma aan het Lorentz Lyceum te Arnhem, begon hij met de studie Geneeskunde aan de Radboud Universiteit in Nijmegen. Tijdens zijn studententijd was Sjaak actief als onderzoeker bij het Centrum voor Plastische Aangezichtschirurgie, waar hij onderzoek deed naar de cosmetische aspecten van de perifere aangezichtsverlamming, alvorens hij in Eindhoven terecht kwam. Gedurende het vierde jaar Geneeskunde is Sjaak gestart met zijn promotieonderzoek. In 2014 behaalde hij zijn arts-examen, na een afsluitende klinische en onderzoeksstage van negen maanden op de afdeling Vaat- en Transplantatiechirurgie van het Radboudumc te Nijmegen. Na het ontvangen van de artsenbul startte Sjaak als artsonderzoeker in het Catharina ziekenhuis te Eindhoven. Gedurende een jaar verrichte hij onderzoek naar de perioperatieve fysiologie bij bariatrische en metabole chirurgie. Daarna heeft hij een periode als ANIOS Intensive Care gewerkt en sinds 1 augustus 2016 is Sjaak werkzaam als ANIOS Chirurgie in het Franciscus Gasthuis en Vlietland in respectievelijk Rotterdam en Schiedam. 
18. LIST OF PUBLICATIONS 

1. Pouwels S, Ingels K., van Heerbeek N., Beurskens C. Cosmetic appreciation of lateralization of peripheral facial palsy: 'preference for left or right, true or mirror image?' Eur Arch Otorhinolaryngol. 2013 Oct 31.

2. Pouwels S, Stokmans RA, Willigendael EM, Nienhuijs SW, Rosman C., van Ramshorst B., Teijink JA. Preoperative exercise therapy for elective major abdominal surgery: A systematic review. Int J Surg. 2013 Dec 8. S1743-9191(13)

3. Pouwels S, Willigendael EM, van Sambeek MR, Nienhuijs SW, Cuypers PW, Teijink JA. Beneficial Effects Of Pre-operative Exercise Therapy in Patients with an Abdominal Aortic Aneurysm: A Systematic Review. Eur J Vasc Endovasc Surg. 2014 Nov 14. pii: S1078-5884(14)

4. Pouwels S, Wit M, Teijink JA, Nienhuijs SW. Aspects of Exercise before or after Bariatric Surgery: A Systematic Review. Obes Facts. 2015 Apr 15;8(2):132-146.

5. Raaijmakers LCH, Pouwels S, Berghuis KA, Nienhuijs SW. Technology-based interventions in the treatment of overweight and obesity: a systematic review. Appetite. $2015 \mathrm{Jul}$ 10: 95:138-151

6. Pouwels S, Kools-Aarts M, Said M, Teijink JA, Smeenk FWJM, Nienhuijs SW.Effects of bariatric surgery on inspiratory muscle strength. SpringerPlus. $2015 \mathrm{Jul}$ 7; 4: 322

7. Özdemir-van Brunschot, DMD, van Laarhoven CJHM, Scheffer GJ, Pouwels S, Wever KE, Warlé MC. What is the evidence for the use of low pressure pneumoperitoneum? A systematic review. Surg Endosc 2015 Aug 15 [Epub ahead of print)

8. Pouwels S, Fiddelaers J, Teijink JA, ter Woorst JF, Siebenga J, Smeenk FWJM. Preoperative exercise therapy in lung surgery patients: a systematic review. Respiratory Medicine. 2015 Aug 15. Pii: S0954-6111 [Epub ahead of print)

9. Kets CM, Ten Broeke SW, Bult P, Caanen BAH, Hoogerbrugge N, de Hullu JA, Links TP, van der Meijden J, Pouwels S, Schieving JH, Vasen HFA, Velthuis MJ, Zonderland HM. De richtlijn PTEN Hamartoom Tumor Syndroom. Nederlands Tijdschrift Voor Oncologie. 2015. 12 (4); 160163

10. Pouwels S, Beurskens CHG, Kleiss IJ, Ingels KJ. Assessing psychological distress in patients with a peripheral facial palsy using the Hospital Anxiety and Depression Scale. J Plast Reconstr Aesthet Surg. 2016 Feb 2. pii: S1748-6815(16)00050-4. doi: 10.1016/j.bjps.2016.01.021. [Epub ahead of print]

11. Van den Houten MM, van Grinsven R, Pouwels S, Yo LS, van Sambeek MR, Teijink JA. Treatment of upper-extremity outflow thrombosis. Phlebology. $2016 \mathrm{Mar} ; 31$ (1 Suppl):28-33. doi: 10.1177/0268355516632661

12. Celik A, Dixon JB, Pouwels S, Celik BO, Karaca FC, Santoro S, Gupta A, Ugale S. Effects of different metabolic states and surgical models on glucose metabolism and secretion of ileal L-cell peptides: a study protocol for a cross-sectional study. BMJ Open. 2016 Mar 14;6(3):e010245. doi: 10.1136/bmjopen-2015-010245.

13. Smelt HJM, Pouwels S, Said M, Berghuis KA, Boer AK, Smulders JF. Comparison between different intramuscular vitamin B12 supplementation regimes: A retrospective matched cohort study. Obes Surg. 2016 May 5. [Epub ahead of print]

14. Pouwels S, Buise MP, Smeenk FWJM, Teijink JA, Nienhuijs SW. Comparative analysis of respiratory muscle strength before and after bariatric surgery using five different predictive 
equations. J Clin Anesth. 2016 Aug;32:172-80. doi: 10.1016/j.jclinane.2016.03.005. Epub 2016 Apr 20.

15. Luijmes RE, Pouwels S, Boonman J. The Effectiveness of Neurofeedback on Cognitive Functioning in Patients with Alzheimer's disease. Neurophysiol Clin. 2016 Jun 30. pii: S09877053(16)30122-8. doi: 10.1016/j.neucli.2016.05.069. [Epub ahead of print]

16. Pouwels S, Beurskens CHG, Luijmes RE, Ingels KJAO. Clinical importance of smiling in patients with a peripheral facial palsy. J Plast Reconstr Aesthet Surg 2016 Sep; 69(9):1305-6. doi: 10.1016/j. bjps.2016.07.001. Epub 2016 Jul 11.

17. Pouwels S, Smeenk FWJM, Manschot L, Lascaris B, Nienhuijs SW, Bouwman RA, Buise MP. Perioperative respiratory care in obese patients undergoing bariatric surgery: implications for clinical practice. Respir Med. 2016 Aug;117:73-80. doi: 10.1016/j.rmed.2016.06.004. Epub 2016 Jun 7.

18. Pouwels S, Hageman D, Gommans, LNM, Willigendael EM, Nienhuijs SW, Scheltinga MR, Teijink JAW. Preoperative exercise therapy in surgical care: a scoping review. J Clin Anesth. 2016 Sep;33:476-90. doi: 10.1016/j.jclinane.2016.06.032

19. Smelt HJM, Pouwels S, Smulders JF. The clinical dilemma of calcium supplementation after bariatric surgery: Calcium citrate or calcium carbonate that is the question? Obes Surg. 2016 Aug 24. [Epub ahead of print]

20. Celik A, Pouwels S, Çağıltay E, Karaca FC, Büyükbozkırlı D, Ugale S. Time to Glycemic Control - an observational study between 3 different operations. Obes Surg. 2016 Aug 26. [Epub ahead of print]

21. Raaijmakers LCH, Pouwels S, Thomassen SEM, Nienhuijs SW. Quality of life and bariatric surgery: a systematic review of short and long term results and comparison with community norms. Eur J Clin Nutr. 2016 Nov 2. doi: 10.1038/ejcn.2016.198. [Epub ahead of print]

22. Smelt HJM, Pouwels S, Smulders JF. Different supplementation regimes to treat perioperative vitamin B12 deficiencies in bariatric surgery: a systematic review. Obes Surg. 2016 Nov 12. [Epub ahead of print]

23. Luijmes RE, Pouwels S, Beurskens CH, Kleiss IJ, Siemann I, Ingels KJ. Quality of life before and after different treatment modalities in peripheral facial palsy: a systematic review. Laryngoscope. 2016 Nov 12. doi: 10.1002/lary.26356. [Epub ahead of print]

24. Bazuin I, Pouwels S, Houterman S, Nienhuijs SW, Smulders JF, Boer AK. Improved and more effective algorithms to screen for nutrient deficiencies after bariatric surgery. Eur J Clin Nutr. 2016 Dec 14. doi: 10.1038/ejcn.2016.254. [Epub ahead of print]

25. Pouwels S, Lascaris B, Nienhuijs SW, Bouwman RA, Buise MP. Short-term changes in cardiovascular hemodynamics in response to bariatric surgery and weight loss using the Nexfin ${ }^{\infty}$ non-invasive continuous monitoring device. Obes Surg. 2017 Jan 30. doi: 10.1007/s11695-0172564-2. [Epub ahead of print] 


\section{ACCEPTED FOR PUBLICATION:}

26. Pouwels S, Stepaniak PS, Buise MP, Bouwman RA, Nienhuijs SW. The RAQET Study: The effect of eating wateR ice After bariatric surgery on the Quality of patiEnT Recovery; a randomised controlled trial. Indian Journal Of Surgery

27. Van Wezenbeek MR, Pouwels S, Buise MP, Smulders JF, Nienhuijs SW, van Montfort G. Conventional care versus fast track care in an unselected group of patients undergoing revisional bariatric surgery. International Journal Of Surgery Open

28. Pouwels S, Dabekausen, YA. Rib stress fractures in pregnancy: a case report and review of literature. Clinical and Experimental Obstetrics and Gynaecology

29. Pouwels S, Said M, Celik A. The necessity of preoperative pulmonary function screening in patients scheduled for bariatric surgery. Journal Of Obesity and Weight Loss Therapy

30. Pouwels S, Lascaris B, Nienhuijs SW, Bouwman RA, Buise MP. Validation of the Nexfin ${ }^{\varpi}$ noninvasive continuous blood pressure monitoring validated against Riva-Rocci/Korotkoff in a bariatric patient population. Journal of Clinical Anesthesia

31. Luijmes RE, Beurskens CHG, Pouwels S, Ingels KJAO. A prospective cohort study assessing differences in cosmetic appreciation of lateralization while smiling in patients with a peripheral facial palsy. Laterality

\section{SUBMITTED:}

32. Lascaris B, Pouwels S, Houthuizen P, Dekker LR, Nienhuijs SW, Bouwman RA, Buise MP. Cardiac structure and function before and after bariatric surgery: A new perspective. European Journal Of Preventive Cardiology

33. Smelt HJM, Pouwels S, Smulders JF. The influence of different calcium and vitamin D supplementation regimes on vitamin D, calcium and parathyroid hormone after bariatric surgery: a pilot study. Digestive Diseases and Sciences

34. Pouwels S, Smelt HJM, Smulders JF. Is inflammation status in post-bariatric patients predictive for their response to vitamin B12 supplementation therapy? International Surgery

35. Smelt HJM, Pouwels S, Celik A, Gupta A, Smulders JF. Assessment of physical fitness after bariatric surgery and its association with protein intake and type of vitamin $\mathrm{D}$ supplementation. European Journal of Physical and Rehabilitation medicine

36. Smelt HJM, Pouwels S, Boer AK, Smulders JF. Sneller opsporen en behandelen van een functionele vitamine B12 deficiëntie na bariatrische chirurgie. Nederlands Tijdschrift voor Voeding en Diëtetiek

37. Stepaniak PS, Pouwels S, van Straten AHM. Early warning system for balancing demand and supply in the operating rooms: a heuristic approach for the cardiothoracic department. European Journal Of Operational Research

38. Kreeftenberg HG, Pouwels S, van der Voort PH. Efficient organisation of the Intensive Care with the focus on quality: The non-physician provider. Critical Care 